MEASUREMENT OF COAGULATING SILVER

AND CARBON AEROSOLS USING A TANDEM

DIFFERENTIAL MOBILITY ANALYZER

A Dissertation
presented to
the Faculty of the Graduate School
at the University of Missouri-Columbia
In Partial Fulfillment
of the Requirements for the Degree
Doctor of Philosophy
DATTHEW PAUL SIMONES
Dr. Sudarshan K. Loyalka, Dissertation Supervisor
DECEMBER 2013


(C) Copyright by Matthew Paul Simones 2013

All Rights Reserved 
The undersigned, appointed by the dean of the Graduate School, have examined the dissertation entitled

\section{MEASUREMENT OF COAGULATING SILVER AND CARBON AEROSOLS USING A TANDEM DIFFERENTIAL MOBILITY ANALYZER}

presented by Matthew Paul Simones,

a candidate for the degree of Doctor of Philosophy,

and hereby certify that, in their opinion, it is worthy of acceptance.

Dr. Sudarshan K. Loyalka

Dr. Tushar K. Ghosh

Dr. Robert V. Tompson

Dr. Mark A. Prelas

Dr. Dabir S. Viswanath 
To Brooke 


\section{ACKNOWLEDGMENTS}

During my time at the Nuclear Science and Engineering Institute (NSEI) I have had the chance to learn, interact, and collaborate with an incredible group of people. I wish to thank my advisor Prof. Sudarshan Loyalka who first invited me to the institute through a summer research opportunity which eventually led to becoming a Ph.D. student and part of his research group. I am grateful for the support and encouragement he has given me to pursue opportunities both within the walls of NSEI and abroad. I would also like to thank my remaining committee members Prof. Tushar Ghosh, Prof. Robert Tompson, Prof. Mark Prelas, and Prof. Dabir Viswanath for their support and providing an open atmosphere conducive to learning. I am also grateful for the financial support from the GAANN fellowship and for the NEUP infrastructure grant (DE-NE0000323) responsible for funding the instrumentation used in this research. Acknowledgement and thanks are also due to the NSEI staff who keep daily operations running smoothly. I would also like to thank Brian Samuels and Michael Klote of Engineering Technical Services for their assistance with component fabrication.

I want to thank my fellow classmates and colleagues for their insightful discussions and light hearted conversations which helped keep me motivated and focused during this endeavor. In particular I would like to thank my friend and office mate Mike Reinig who made a small office space enjoyable and unforgettable.

Of course I am forever grateful to my mom, dad and family who provide eternal love and support. Especially to my wife who has been incredibly patient while I complete my academic goals. 


\section{TABLE OF CONTENTS}

ACKNOWLEDGMENTS ................................................................................ ii

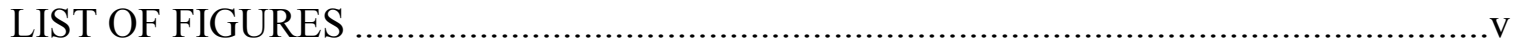

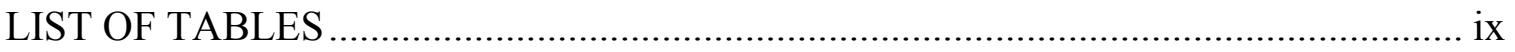

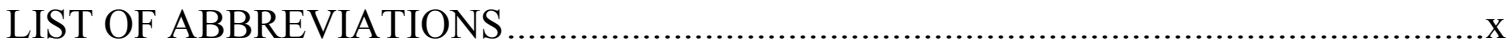

NOMENCLATURE .........................................................................................

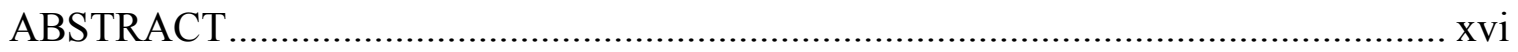

Chapter

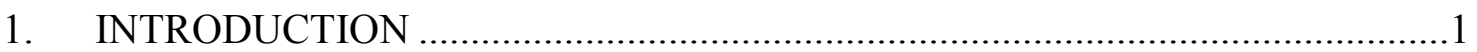

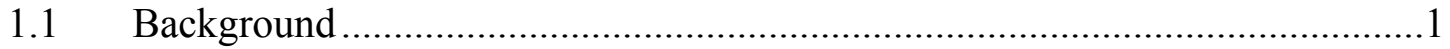

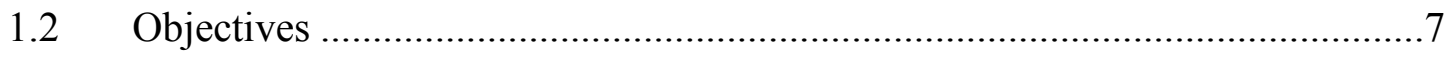

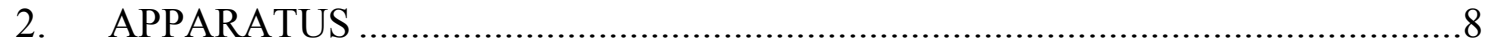

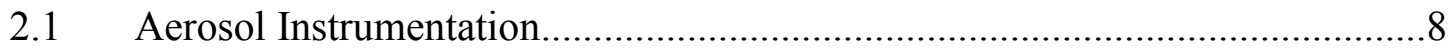

2.1.1 Differential Mobility Analyzer (DMA) .......................................

2.1.2 Scanning Mobility Particle Sizer (SMPS) ......................................15

2.1.3 Tandem Differential Mobility Analyzer (TDMA) ..........................17

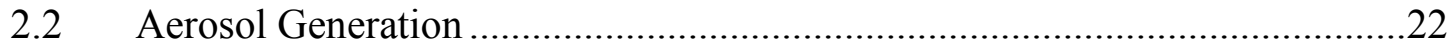

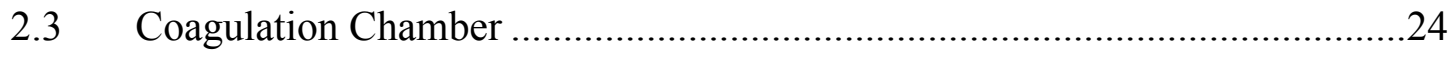

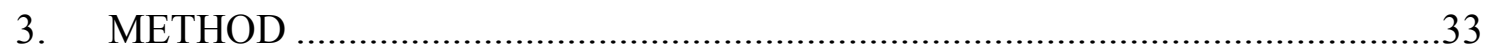

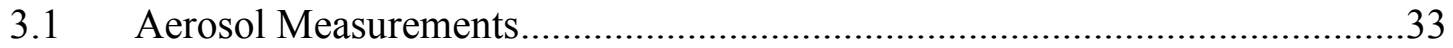

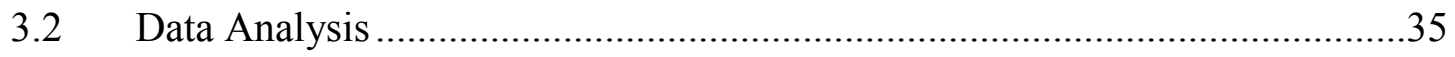

3.3 Numerical Simulations of Aerosol Coagulation ...........................................37 
3.3.1 Sectional Method

3.3.2 Direct Simulation Monte Carlo Method ..........................................40

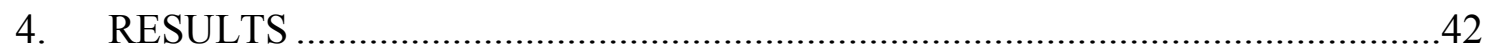

4.1 Measured Size and Charge Distributions...............................................42

4.1.1 Spark Generated Silver Nanoparticles ........................................42

4.1.2 Spark Generated Carbon Nanoparticles.......................................46

4.2 Comparison of Sectional Method with Measurements .................................50

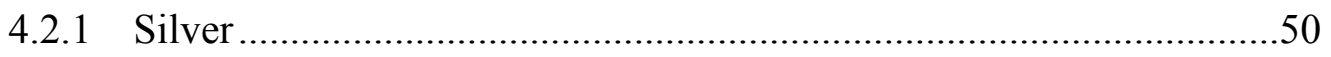

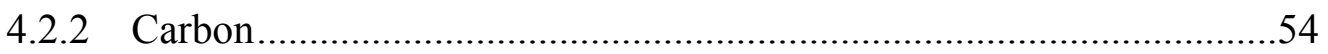

4.3 Comparison of DSMC Method with Measurements ...................................58

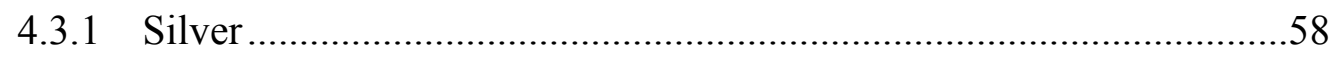

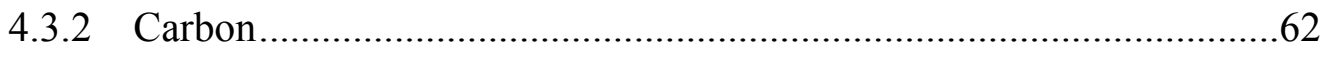

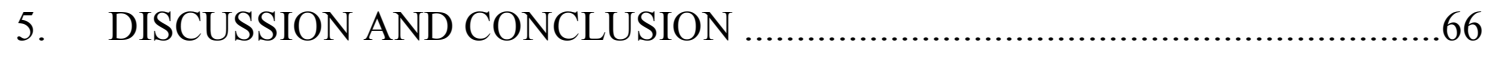

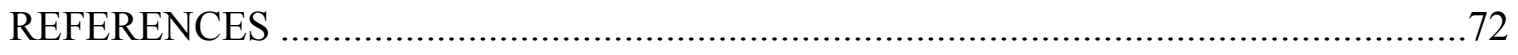

\section{APPENDICES}

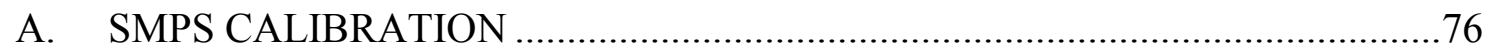

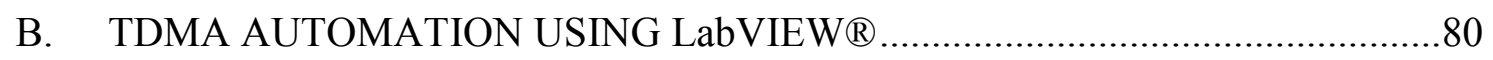

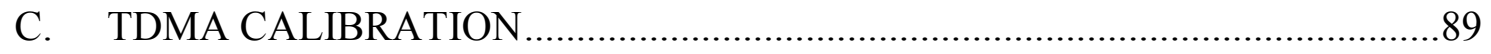

D. SAMPLING PROBE CAP AND PORT CAP DESIGN .................................94

E. DIFFUSER AND NOZZLE DESIGN .......................................................97

F. COAGULATION CHAMBER DESIGN …........................................... 102

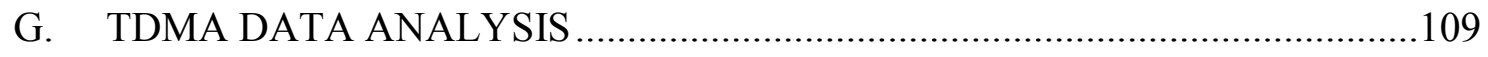

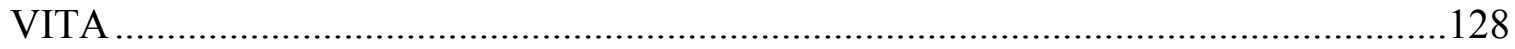




\section{LIST OF FIGURES}

Figure

Page

1. Schematic diagram of a typical cylindrical DMA showing trajectory of a charged particle with the characteristic electrical mobility, $Z_{p}^{*}$.

2. Comparison of non-diffusing $\left(\Omega_{n d}\right)$ and diffusing $\left(\Omega_{d}\right)$ transfer functions as a function of dimensionless electrical mobility, $\widetilde{Z}_{p}$, for a particle diameter of (a) $5 \mathrm{~nm}$ and (b) $100 \mathrm{~nm}$.

3. Flow diagram showing the classification of a sampled aerosol by electrical mobility using DMA-1 and the subsequent measurement of the corresponding size distribution using the SMPS.

4. TEM images of spark generated (a) graphite (100 nm scale) and (b) silver (50 nm scale).

5. Schematic of the experimental setup for (a) silver and (b) carbon aerosol size and charge distribution measurements.

6. Schematic cross sectional view of aerosol sampling probe and dilution device (ejector pump).

7. Measured size and charge distribution for spark generated silver nanoparticles at coagulation chamber sampling port $\# 1(\mathrm{t}=0 \mathrm{sec})$.

8. Measured size and charge distribution for spark generated silver nanoparticles at coagulation chamber sampling port $\# 2(\mathrm{t}=26.2 \mathrm{sec})$.

9. Measured size and charge distribution for spark generated silver nanoparticles at coagulation chamber sampling port \#3 $(\mathrm{t}=78.6 \mathrm{sec})$.

10. Measured size and charge distribution for spark generated silver nanoparticles at coagulation chamber sampling port \#4 $(\mathrm{t}=183.3 \mathrm{sec})$.

11. Measured size and charge distribution for spark generated silver nanoparticles at coagulation chamber sampling port $\# 5(\mathrm{t}=392.8 \mathrm{sec})$. 
12. Measured size and charge distribution for spark generated carbon nanoparticles at coagulation chamber sampling port \#1 $(\mathrm{t}=0 \mathrm{sec})$.

13. Measured size and charge distribution for spark generated carbon nanoparticles at coagulation chamber sampling port $\# 2(\mathrm{t}=26.2 \mathrm{sec})$.

14. Measured size and charge distribution for spark generated carbon nanoparticles at coagulation chamber sampling port $\# 3(\mathrm{t}=78.6 \mathrm{sec})$.

15. Measured size and charge distribution for spark generated carbon nanoparticles at coagulation chamber sampling port \#4 $(\mathrm{t}=183.3 \mathrm{sec})$.

16. Measured size and charge distribution for spark generated carbon nanoparticles at coagulation chamber sampling port $\# 5(\mathrm{t}=392.8 \mathrm{sec})$.

17. Comparison between the measured size and charge distribution for spark generated silver nanoparticles at coagulation chamber sampling port \#1 $(\mathrm{t}=0 \mathrm{sec})$ and the coagulation model using the sectional method .51

18. Comparison between the measured size and charge distribution for spark generated silver nanoparticles at coagulation chamber sampling port \#2 $(\mathrm{t}=26.2 \mathrm{sec})$ and the coagulation model using the sectional method .51

19. Comparison between the measured size and charge distribution for spark generated silver nanoparticles at coagulation chamber sampling port \#3 $(\mathrm{t}=78.6 \mathrm{sec})$ and the coagulation model using the sectional method

20. Comparison between the measured size and charge distribution for spark generated silver nanoparticles at coagulation chamber sampling port \#4 $(\mathrm{t}=183.3 \mathrm{sec})$ and the coagulation model using the sectional method

21. Comparison between the measured size and charge distribution for spark generated silver nanoparticles at coagulation chamber sampling port \#5 $(\mathrm{t}=392.8 \mathrm{sec})$ and the coagulation model using the sectional method

22. Comparison between the measured size and charge distribution for spark generated carbon nanoparticles at coagulation chamber sampling port \#1 $(\mathrm{t}=0 \mathrm{sec})$ and the coagulation model using the sectional method

23. Comparison between the measured size and charge distribution for spark generated carbon nanoparticles at coagulation chamber sampling port \#2 $(\mathrm{t}=26.2 \mathrm{sec})$ and the coagulation model using the sectional method

24. Comparison between the measured size and charge distribution for spark generated carbon nanoparticles at coagulation chamber sampling port \#3 $(\mathrm{t}=78.6 \mathrm{sec})$ and the coagulation model using the sectional method .56 
25. Comparison between the measured size and charge distribution for spark generated carbon nanoparticles at coagulation chamber sampling port \#4 $(\mathrm{t}=183.3 \mathrm{sec})$ and the coagulation model using the sectional method ..... .56

26. Comparison between the measured size and charge distribution for spark generated carbon nanoparticles at coagulation chamber sampling port \#5 $(\mathrm{t}=392.8 \mathrm{sec})$ and the coagulation model using the sectional method

27. Comparison between the measured size and charge distribution for spark generated silver nanoparticles at coagulation chamber sampling port \#1 $(\mathrm{t}=0 \mathrm{sec})$ and the coagulation model using the DSMC method

28. Comparison between the measured size and charge distribution for spark generated silver nanoparticles at coagulation chamber sampling port \#2 $(\mathrm{t}=26.2 \mathrm{sec})$ and the coagulation model using the DSMC method

29. Comparison between the measured size and charge distribution for spark generated silver nanoparticles at coagulation chamber sampling port \#3 $(\mathrm{t}=78.6 \mathrm{sec})$ and the coagulation model using the DSMC method

30. Comparison between the measured size and charge distribution for spark generated silver nanoparticles at coagulation chamber sampling port \#4 $(\mathrm{t}=183.3 \mathrm{sec})$ and the coagulation model using the DSMC method

31. Comparison between the measured size and charge distribution for spark generated silver nanoparticles at coagulation chamber sampling port \#5 $(\mathrm{t}=392.8 \mathrm{sec})$ and the coagulation model using the DSMC method

32. Comparison between the measured size and charge distribution for spark generated carbon nanoparticles at coagulation chamber sampling port \#1 $(\mathrm{t}=0 \mathrm{sec})$ and the coagulation model using the DSMC method

33. Comparison between the measured size and charge distribution for spark generated carbon nanoparticles at coagulation chamber sampling port \#2 $(\mathrm{t}=26.2 \mathrm{sec})$ and the coagulation model using the DSMC method

34. Comparison between the measured size and charge distribution for spark generated carbon nanoparticles at coagulation chamber sampling port \#3 $(\mathrm{t}=78.6 \mathrm{sec})$ and the coagulation model using the DSMC method

35. Comparison between the measured size and charge distribution for spark generated carbon nanoparticles at coagulation chamber sampling port \#4 $(\mathrm{t}=183.3 \mathrm{sec})$ and the coagulation model using the DSMC method 
36. Comparison between the measured size and charge distribution for spark generated carbon nanoparticles at coagulation chamber sampling port \#5 $(\mathrm{t}=392.8 \mathrm{sec})$ and the coagulation model using the DSMC method

A1. Aerosol generator for SMPS particle size calibration using aqueous solution of monodisperse particles.

A2. SMPS measurement of monodisperse PSL spheres of diameter (a) $65 \mathrm{~nm}$ and (b) $99 \mathrm{~nm}$.

B1. Front panel of LabVIEW® program for TDMA.

B2. LabVIEW ${ }^{\circledR}$ program for TDMA (sequence 0 of 6 ).

B3. LabVIEW ${ }^{\circledR}$ program for TDMA (sequence 1 of 6 ).

B4. LabVIEW® program for TDMA (sequence 2 of 6 ).

B5. LabVIEW ${ }^{\circledR}$ program for TDMA (sequence 3 of 6 ).

B6. LabVIEW ${ }^{\circledR}$ program for TDMA (sequence 4 of 6 ).

B7. LabVIEW ${ }^{\circledR}$ program for TDMA (sequence 5 of 6 ).

B8. LabVIEW ${ }^{\circledR}$ program for TDMA (sequence 6 of 6 ).

C1. Aerosol generation apparatus including pharmaceutical nebulizer, air duct, and aerosol sampling probe $(L=200 \mathrm{~mm})$.

C2. Schematic diagram of (a) SMPS measurements for charged neutralized aerosol

(b) TDMA measurements for charge neutralized aerosol.

C3. Measured charge fractions for charge neutralized $\mathrm{NaCl}$ aerosol with comparisons against the corrected Fuchs $\left(f_{W}\right)$ and Boltzmann $\left(f_{B}\right)$ charge distributions.

D1. Engineering drawing of the coagulation chamber sampling port cap.

D2. Engineering drawing of the coagulation chamber sampling probe cap.

E1. COMSOL axisymmetric flow model results of diffuser design with deflectors. .99

E2. Engineering drawing of coagulation chamber large angle diffuser with diverters.

E3. Engineering drawing of coagulation chamber nozzle. 100 


\section{LIST OF TABLES}

Table

Page

1. Summary of charged aerosol coagulation experiments.

2. Summary of design criteria and selected parameters.

3. Summary of instrument settings used for both silver and carbon aerosol measurements.

4. Least squares results for TDMA data collected for positively charged spark generated carbon nanoparticles at size bin $d_{p, i}=27.9 \mathrm{~nm}$.

C1. Instrument settings and parameters used for TDMA and SMPS

measurements. 


\section{LIST OF ABBREVIATIONS}

$\begin{array}{ll}\text { AIM } & \text { Aerosol Instrument Manager } \\ \text { COA } & \text { Constant Output Atomizer } \\ \text { CPC } & \text { Condensation Particle Counter } \\ \text { DMA } & \text { Differential Mobility Analyzer } \\ \text { DSMC } & \text { Direct Simulation Monte Carlo } \\ \text { DOP } & \text { Dioctyl Phthalate } \\ \text { FDM } & \text { Fused Deposition Modeling } \\ \text { HTGR } & \text { High Temperature Gas-cooled Reactor } \\ \text { NSEI } & \text { Nuclear Science and Engineering Institute } \\ \text { PSL } & \text { Polystyrene Latex } \\ \text { SMPS } & \text { Scanning Mobility Particle Sizer } \\ \text { TDMA } & \text { Tandem Differential Mobility Analyzer } \\ \text { TEM } & \text { Transmission Electron Microscopy } \\ \end{array}$




\title{
NOMENCLATURE
}

\author{
Subscripts \\ asp aspiration \\ particle size index \\ $j \quad$ particle size index \\ p particle \\ tot total \\ Superscripts \\ $i$ \\ particle size index \\ $j \quad$ particle size index \\ Italic letters \\ $A_{\gamma}$ \\ parameter for diffusing transfer function $\Omega_{d}$ \\ $B$ \\ particle mechanical mobility, $C_{c}(K n) / 3 \pi \mu d_{p}$ \\ $C_{c}(K n) \quad$ Cunningham slip correction factor, \\ $1+K n[1.142+0.558 \exp (-0.999 / K n)]$ \\ $d_{p} \quad$ particle diameter \\ $d \quad$ sampling probe inlet diameter \\ D \\ coagulation chamber tube diameter
}




\begin{tabular}{|c|c|}
\hline$\tilde{D}$ & dimensionless particle diffusivity, $=4 \pi \mathrm{L} D /\left(Q_{e}+Q_{s h}\right)$ \\
\hline$e$ & elementary unit of charge, $=1.60217 \times 10^{-19} \mathrm{C}$ \\
\hline$F$ & parameter for Brownian coagulation kernel $\beta^{i, j}$ \\
\hline$F_{\gamma}$ & integral quantity for evaluation of $\Omega_{d}$ \\
\hline$g$ & gravitational acceleration, $=9.80665 \mathrm{~m} / \mathrm{s}^{2}$ \\
\hline$g_{i}$ & $=\frac{1}{3 d_{p, i} l_{i}}\left|\left(d_{p, i}+\ell_{i}\right)^{3}-\left(d_{p, i}^{2}+l_{i}^{2}\right)^{3 / 2}\right|-d_{p, i}$ \\
\hline$g_{i, j}$ & $=\sqrt{g_{i}^{2}+g_{j}^{2}}$ \\
\hline$G_{D M A}$ & dimensionless DMA geometry and flow factor \\
\hline$I_{\gamma}$ & integral quantity for the evaluation of $\Omega_{d}$ \\
\hline$k_{B}$ & Boltzmann constant, $=1.38065 \times 10^{-23} \mathrm{~J} / \mathrm{K}$ \\
\hline$K_{0}$ & coagulation coefficient, $=4 \pi d_{p} D$ \\
\hline$L$ & $\begin{array}{l}\text { length between DMA polydisperse inlet and sampling window, } \\
=44.369 \mathrm{~cm}\end{array}$ \\
\hline$L_{h}$ & entry length of the hydrodynamic boundary layer \\
\hline$L_{\mathscr{D}}$ & entry length of concentration boundary layer \\
\hline$m$ & particle mass \\
\hline$n$ & number concentration; distribution function \\
\hline$n_{e}$ & number of elementary units of charge carried by particle \\
\hline$n_{e}^{\max }$ & maximum number of elementary units of charge carried by particle \\
\hline$N$ & total number of particles \\
\hline$p_{e}$ & number of elementary units of charge carried by particle \\
\hline
\end{tabular}




\begin{tabular}{|c|c|}
\hline$P$ & particle penetration \\
\hline$q_{e}$ & number of elementary units of charge carried by particle \\
\hline$Q$ & volumetric flow rate \\
\hline$Q_{a}$ & DMA polydisperse volumetric flow rate \\
\hline$Q_{e}$ & DMA main excess volumetric flow rate \\
\hline$Q_{s}$ & DMA monodisperse volumetric flow rate \\
\hline$Q_{s h}$ & DMA sheath air volumetric flow rate \\
\hline$R_{1}$ & inner radius of DMA annular space, $=0.937 \mathrm{~cm}$ \\
\hline$R_{2}$ & outer radius of DMA annular space, $=1.961 \mathrm{~cm}$ \\
\hline$T$ & gas temperature \\
\hline$u$ & gas velocity \\
\hline $\bar{u}$ & mean gas velocity \\
\hline$U$ & sampling velocity \\
\hline$U_{0}$ & free stream sampling velocity \\
\hline$V$ & DMA collector rod voltage \\
\hline$V_{t s}$ & terminal settling velocity, $=\tau g$ \\
\hline$V_{i}$ & $=\sqrt{8 k_{B} T / \pi m_{i}}$ \\
\hline $\bar{V}_{i, j}$ & $=\sqrt{V_{i}^{2}+V_{j}^{2}}$ \\
\hline$\Delta z_{a}$ & DMA polydisperse aerosol entrance slit width \\
\hline$\Delta z_{s}$ & DMA monodisperse aerosol exit slit width \\
\hline$Z_{p}$ & particle electrical mobility, $=n e C_{c}(K n) / 3 \pi \mu d_{p}$ \\
\hline
\end{tabular}


$Z_{p}^{*}$

$\Delta Z_{p}$

$\widetilde{Z}_{p}$

Script letters

D

$l_{i}$

Greek letters

$\beta$

$\beta_{\mathrm{p}, \mathrm{q}}^{\mathrm{i}, \mathrm{j}}$

$\beta^{i, j}$

$\beta_{p, q}$

$\gamma$

$\delta$

$\varepsilon(x)$

$\varepsilon_{0}$

$\eta$

$\kappa$

$\kappa_{a}$
DMA characteristic (transfer function centroid) electrical mobility

DMA transfer function base half width

dimensionless particle electrical mobility, $=Z_{p} / Z_{p}^{*}$ particle diffusion coefficient, $=k_{B} T C_{c}(K n) / 3 \pi \mu d_{p}$ $=8 \mathcal{D}_{i} / \pi V_{i}$

$=\left(Q_{s}+Q_{a}\right) /\left(Q_{e}+Q_{s h}\right) ;$ Fuchs coagulation correction factor

modified Brownian coagulation kernel, $=\beta^{i, j} \beta_{p, q}$

Brownian coagulation kernel, $=2 \pi\left(\mathscr{D}_{i}+\mathscr{D}_{j}\right)\left(\gamma_{i} d_{p, i}+\gamma_{j} d_{p, j}\right) / F$

Brownian coagulation kernel Coulombic correction factor

$=\left(R_{1} / R_{2}\right)^{2}$

$=\left(Q_{s}-Q_{a}\right) /\left(Q_{s}+Q_{a}\right)$

$=x \cdot \operatorname{erf}(x)+\exp \left(-x^{2}\right) / \sqrt{\pi}$

permittivity of free space, $=8.85419 \times 10^{-12} \mathrm{C}^{2} /\left(\mathrm{N} \cdot \mathrm{m}^{2}\right)$

transport efficiency

dimensionless length

$\Delta z_{a} / L$ 


$\begin{array}{ll}\kappa_{s} & \Delta z_{s} / L \\ \mu & \text { mean free path for gas molecules } \\ \rho & \text { dynamic gas viscosity } \\ \rho_{p} & \text { gas density } \\ \sigma & \text { particle density } \\ \tau & \text { dimensionless DMA diffusion parameter, }=\sqrt{G_{D M A} \widetilde{D}} \\ \Omega^{\prime} & \text { Darticle relaxation time, }=\rho_{p} d_{p}^{2} C_{c}(K n) / 18 \mu \\ \Omega_{d} & \text { diffusing transfer function } \\ \Omega_{n d} & \text { non-diffusing transfer function }\end{array}$

Dimensionless numbers

Kn Knudsen number, $=2 \lambda / d_{p}$

$P e_{L} \quad$ Péclet number, $=L \bar{u} / \mathscr{D}$

$\operatorname{Re}_{L} \quad$ Reynolds number, $=\rho \bar{u} L / \mu$

Sc Schmidt number, $=\mu / \rho \mathcal{D}$

$S t k_{L, U} \quad$ Stokes number, $=\tau U / L$ 


\begin{abstract}
One of the unresolved technical issues associated with the high temperature gas-cooled reactor (HTGR) is the production of carbonaceous dust (e.g. by abrasion, corrosion, radiation damage, gas-to-particle conversion) and the subsequent transport of sorbed fission products via aerosol transport. Diffusion charging and/or self-charging of these aerosols is likely to occur which will affect how the aerosol evolves in time and ultimately deposits on surfaces. At present, nuclear reactor safety codes, such as MELCOR, do not account for these electrostatic effects and there is currently no consensus on their importance. Further experimentation and modeling of these effects are therefore important and ongoing to resolve these issues. The purpose of this research is to experimentally investigate the coagulation of charged aerosols closely associated with HTGRs by measuring the evolution of size and charge distributions over time and to compare the experimental results with available numerical models. Measurements have been completed for both silver and carbon ultrafine aerosols using a tandem differential mobility analyzer and an open flow coagulation chamber with a residence time of nearly 400 seconds. Results for both aerosols indicate that coagulation occurs faster than predicted by the model, at times differing by an order of magnitude. Overall, the apparatus developed here will support future coagulation studies of charged ultrafine aerosols at the Nuclear Science and Engineering Institute by providing data for validation of computer codes and guiding model development.
\end{abstract}




\section{Chapter 1}

\section{INTRODUCTION}

\subsection{Background}

One of the unresolved technical issues associated with the high temperature gas-cooled reactor (HTGR) is the production of carbonaceous dust (e.g. by abrasion, corrosion, radiation damage, gas-to-particle conversion) and the subsequent transport of sorbed fission products via aerosol transport. Diffusion charging and/or self-charging of these aerosols is also likely to occur which will affect how the aerosol evolves in time and ultimately deposits on surfaces. Diffusion charging of aerosols occurs by attachment of gas-phase ions generated within the intense radiation field of the reactor environment. Since negative ions are more likely to have higher mobilities, the aerosol particles may assume a net negative charge. Conversely, self-charging of radioactive aerosols leads to a net positive charge either by the emission of beta electrons or the emission of secondary electrons caused by ionization during alpha decay. The charge state of the aerosol is then dependent on the balance between the rate of self-charging and the rate of neutralization by gas-phase ions (Gensdarmes et al. 2001). The asymmetric charging of aerosol particles, in general, can lead to electrostatic dispersion which slows down coagulation, inhibiting particle growth and retarding the deposition of particles by both gravitational settling and inertial impaction on structures. However, it has also been shown theoretically that radioactive aerosols can either enhance or reduce coagulation rates depending on the type of radioactive decay (i.e. $\alpha, \beta$ ), the relative sizes of the interacting aerosols, and the concentration and mobility of positive and negative background ions 
(Clement et al. 1995). Furthermore, as charged particles deposit on surfaces, charge build-up and mirror image charge effects can prevent further deposition of charged particles (Morris 2008). Presently, nuclear reactor safety codes, such as MELCOR, do not account for these electrostatic effects and there is currently no consensus on their importance (Humrickhouse 2011). Further experimentation and modeling of these effects are therefore important and ongoing to resolve these issues.

Brownian coagulation has been investigated extensively to examine the applicability of the Smoluchowski coagulation model and its extension to more complex situations (Davies 1979; Shaw 1978). More generally, theoretical modeling and numerical simulations of the general dynamic equation to describe aerosol coagulation has also been carried out to determine the change in the particle distribution function and its dependence on various parameters such as polydispersity, charge, and particle shape (Friedlander 2000; Hidy and Brock 1970; Williams and Loyalka 1991). However, with numerical simulations of increasing model complexity, there is a deficiency in experimental data to validate the results (Palsmeier and Loyalka 2013).

With respect to electrostatic charge effects on aerosol coagulation, a number of experimental studies have been carried out as summarized in Table 1. Kennedy (1916) studied aerosol sampled from a Bunsen flame and used a "gasometer" (elastomeric chamber) to store and age the aerosol. The total number concentration was then measured over time with an Aitken counter and the effect of charge was investigated by using an electrostatic precipitator to remove charged particles. A noticeable difference between charged and uncharged particles was not observed and both were in fair agreement with the Smoluchowski equation during the initial stages of the measurements. After large 
times the coagulation coefficient seemed to increase, likely due to particle loss to surfaces which was not accounted for. Gillespie and Langstroth (1952) investigated the effects of particle charge on aerosol coagulation using silica powder inside an enclosed chamber and a charging grid to alter the charge on the aerosol. Number concentration was measured at different times by sampling a known volume from the chamber, collecting particles on a glass slide using a thermal precipitator, and counting the particles under a microscope. It was found that altering the charge on the aerosol appreciably changed both coagulation rates and diffusion losses. Rosinski et al. (1962) compared the coagulation rate of non-radioactive and radioactive aerosols generated using an exploding wire technique. Particles were collected from a chamber using a String-Ficklen oscillating thermal precipitator for examination using dark-field and electron microscopy. Nonradioactive aerosols were found to have higher coagulation rates than slightly radioactive aerosols, however with increased radioactivity the coagulation was greatly increased during early stages. Devir ((Wienstock) (1967)) sampled monodisperse dioctyl phthalate (DOP) from a closed chamber and used a Millikan cell with a camera to determine charge distributions by measuring particle trajectories. The charged aerosol was found to have $1 e-3 e$ charges per particle and it was concluded that small electric charges have a negligible effect on the coagulation rate. Xiang and Colbeck (1993) conducted coagulation experiments on charged, monodisperse aerosol in an enclosed chamber using a differential mobility analyzer (DMA) to measure particle charge and a condensation nucleus counter to measure particle concentration. Compared to uncharged particles, unipolar particles were found to have suppressed coagulation rates and symmetrical, bipolar particles were found to have higher coagulation rates. Katzer et al. (2001) 
performed a series of experiments with monodisperse aerosol $\left(\mathrm{TiO}_{2}\right)$ in a flowing coagulation tube in which the particle concentration and particle charge were followed over time using a scanning mobility particle sizer (SMPS). They found that the experimental results were in good agreement with theory when both particle charge and aggregate size were accounted for. They also determined that the effect of charge on the collision frequency is much larger than the effect of aggregate size. Maisels et al. (2002) used a mixture of monomobile (equivalent electrical mobility as selected by DMA), oppositely charged $\mathrm{PbS}$ and $\mathrm{Ag}$ aerosol and measured the change in the neutrally charged fraction for different aggregation tube lengths (i.e. residence times) using a condensation particle counter (CPC). The measurements were found to be in agreement with Fuchs' theory (Fuchs 1964). Coagulation of polydisperse, bipolarly charged aerosols were also measured and found to be in good agreement with uncharged particle theory after a dimensionless representation was used. Subramanian et al. (2008) conducted coagulation experiments using sodium aerosol within a closed chamber and examined the effects of a surrounding gamma field on the coagulation rate. The authors found that the gamma field increased the coagulation coefficient by nearly one order of magnitude, indicating that the bipolar charging due to the radiation field enhances Brownian coagulation. The studies mentioned above are all limited in their ability to specifically monitor the evolution of both size and charge distributions of the aerosol particles under investigation. This is an important aspect for validation of numerical models involving charged polydisperse aerosols.

Recently, a new method for measuring the size and charge distribution simultaneously has been reported using a tandem differential mobility analyzer (TDMA) 
(Chadha et al. 2012; Kim et al. 2005; Maricq 2005; Simones et al. 2011; Simones et al. Submitted). The first DMA is operated without a charge neutralizer so that particles are classified by electrical mobility. These particles are then charge neutralized and classified again by size using the second DMA. The size and charge distribution is then recovered through a data inversion process. This experimental technique is tailored specifically for nanosized particles where optical techniques of size measurement are not applicable. Maricq has applied this technique to investigate the electrical characteristics and coagulation of soot sampled from premixed flames and has found that the measurements agree with theoretical models accounting for multiply charged polydisperse particles (Maricq 2005; 2006). 


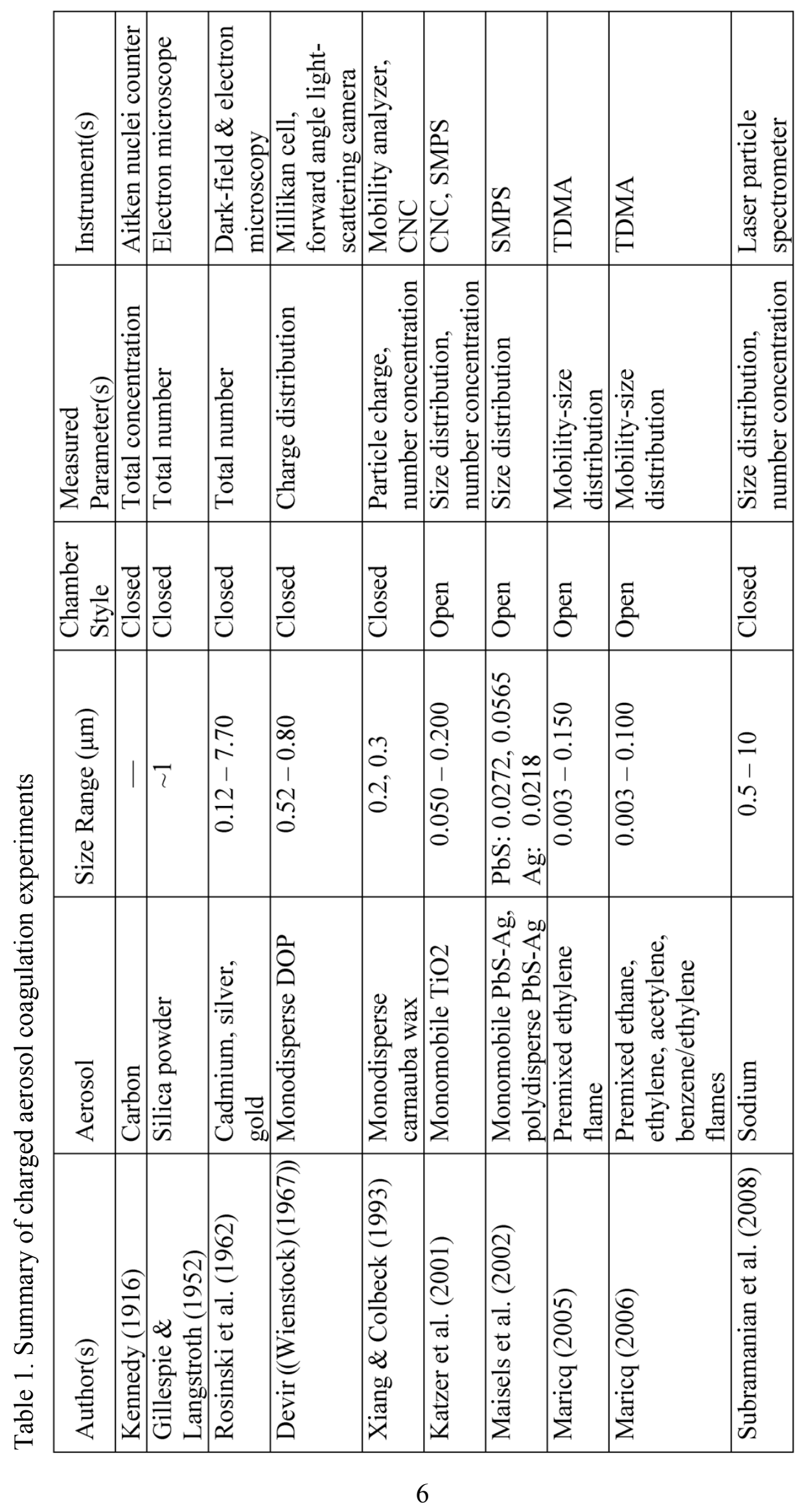




\section{$1.2 \quad$ Objectives}

The purpose of this research is to experimentally investigate the coagulation of charged aerosols closely associated with HTGRs by measuring the evolution of size and charge distributions over time and to compare the experimental results with available numerical codes. To accomplish these goals the following tasks will be completed:

1. Set up TDMA using TSI 3080 Electrostatic Classifier and TSI 3936 Scanning Mobility Particle Sizer ${ }^{\circledR}$ (TSI 3080 Electrostatic Classifier + TSI 3775 Condensation Particle Counter)

2. Automate operation and data collection of TDMA using LabVIEW®

3. Design a coagulation chamber which allows the evolution of a test aerosol to be measured while meeting the restrictions of the TDMA measurement system

4. Perform coagulation measurements on spark generated test aerosols including carbon and silver

5. Perform data inversion to obtain aerosol size and charge distributions

6. Compare experimental results with available numerical models, examine differences, and suggest model improvements as necessary 


\section{Chapter 2}

\section{APPARATUS}

\subsection{Aerosol Instrumentation}

In this study, two principle instruments were used to quantitatively measure the size and charge distribution of aerosol particles, namely the SMPS and the TDMA. Together the SMPS and TDMA provide aerosol size distributions as a function of particle electrical mobility, which then require further analysis to determine the charge distribution. Fundamental to both the SMPS and TDMA is the DMA which is the device used to sort or "classify" particles by electrical mobility. In the following sections the operating principle of the DMA, SMPS, and TDMA will be reviewed in addition to descriptions of the specific SMPS and TDMA instruments used in this study.

\subsubsection{Differential Mobility Analyzer (DMA)}

Differential mobility analyzers, sometimes referred to as differential mobility classifiers, are typically used to either produce a source of monodisperse aerosol or in obtaining aerosol size distributions. Both cylindrical and radial DMA configurations are in common use, however in this study only the cylindrical DMA was used and so discussion of the radial DMA will be omitted. As shown in Figure 1, the cylindrical DMA consists of an annular flow region formed by two coaxial cylindrical electrodes. Polydisperse aerosol, $Q_{a}$, enters at the top of the DMA through a narrow slit of width $\Delta z_{a}$ along the periphery of the annulus adjacent to the outer electrode and is separated from the inner electrode (i.e. collector rod) by a particle-free sheath flow, $Q_{s h}$. Both streams flow laminarly downward 
without mixing and exit at the bottom of the DMA either as the main excess flow, $Q_{e}$, or through the monodisperse aerosol outlet of width $\Delta z_{s}$ on the collector rod, $Q_{s}$. With the outer electrode grounded, a variable voltage $( \pm 0-10 \mathrm{kV})$ is applied to the inner electrode forming a radial electric field in the annular region. Charged particles which enter with the polydisperse flow will migrate radially within the electric field as they are carried at gas velocity in the axial direction. Particles of the appropriate polarity will migrate toward the collector rod and traverse the sheath flow, either partially or completely, with each particle having a unique trajectory within the DMA depending on its particular electrical mobility, $Z_{p}$, given by (Flagan 2001)

$$
Z_{p}=\frac{n_{e} e C_{c}(K n)}{3 \pi \mu d_{p}}
$$

where $n_{e}$ is the number of elementary units of charge carried by the particle, $e$ is the elementary unit of charge, $\mu$ is the dynamic gas viscosity, $d_{p}$ is the particle diameter, and $C_{c}(K n)$ is the Cunningham slip correction factor as a function of Knudsen number (Baron and Willeke 2001)

$$
C_{c}(K n)=1+K n\left[1.142+0.558 \exp \left(\frac{-0.999}{K n}\right)\right] \text { (for solid particles). }
$$

For a given combination of flow rates and applied voltage, particles within a narrow range of the characteristic electrical mobility, $Z_{p}^{*} \pm \Delta Z_{p}$, will fall within the sampling window forming a monodisperse aerosol. Particles with higher electrical mobility will be deposited on the collector rod and particles with lower electrical mobility will exit the DMA with the excess flow. The characteristic electrical mobility for the cylindrical DMA is given by (Flagan 2001) 


$$
Z_{p}^{*}=\frac{\left(Q_{s h}+Q_{e}\right)}{4 \pi V L} \ln \left(\frac{R_{2}}{R_{1}}\right)
$$

where $V$ is voltage on the collector rod, $L$ is the length between the polydisperse inlet and the sampling window, and $R_{1}$ and $R_{2}$ are the inner and outer radii of the annular space, respectively. The mobility range can be expressed as, neglecting particle diffusion,

$$
\Delta Z_{p}=\frac{\left(Q_{a}+Q_{s}\right)}{\left(Q_{s h}+Q_{e}\right)} Z_{p}^{*},
$$

however not all particles within this range have equal probability of passage through the DMA. The probability that a particle entering the DMA with the polydisperse aerosol flow will exit with the monodisperse flow is given by the transfer function $\Omega\left(Z_{p}, Z_{\mathrm{p}}^{*}\right)$, which for non-diffusing particles can be expressed as, in non-dimensional form (Stolzenburg and McMurry 2008),

$$
\begin{array}{r}
\Omega_{n d}\left(\widetilde{Z}_{p}, \beta, \delta\right)=\frac{1}{2 \beta(1-\delta)} \mid\left[\widetilde{Z}_{p}-(1+\beta)|+| \widetilde{Z}_{p}\right. \\
\left.-(1-\beta)|-| \widetilde{Z}_{p}-(1+\beta \delta)|-| \widetilde{Z}_{p}-(1-\beta \delta) \mid\right]
\end{array}
$$

with

$$
\begin{aligned}
& \widetilde{Z}_{p}=Z_{p} / Z_{p}^{*} \\
& \beta=\left(Q_{s}+Q_{a}\right) /\left(Q_{e}+Q_{s h}\right) \\
& \delta=\left(Q_{s}-Q_{a}\right) /\left(Q_{s}+Q_{a}\right) .
\end{aligned}
$$

In general diffusion effects are not negligible, especially for particles in the ultrafine range $\left(d_{p}<100 \mathrm{~nm}\right)$ as used in this study. Stolzenburg and McMurry (2008) present results for a diffusing transfer function assuming a Gaussian spreading profile expressed as, in non-dimensional form, 


$$
\begin{aligned}
& \Omega_{d}\left(\widetilde{Z}_{p}, \beta, \delta, \sigma\right)=\frac{\sigma}{\sqrt{2} \beta(1-\delta)}\left[\varepsilon\left(\frac{\widetilde{Z}_{p}-(1+\beta)}{\sqrt{2} \sigma}\right)\right. \\
& \left.\quad+\varepsilon\left(\frac{\widetilde{Z}_{p}-(1-\beta)}{\sqrt{2} \sigma}\right)-\varepsilon\left(\frac{\widetilde{Z}_{p}-(1+\beta \delta)}{\sqrt{2} \sigma}\right)-\varepsilon\left(\frac{\widetilde{Z}_{p}-(1-\beta \delta)}{\sqrt{2} \sigma}\right)\right]
\end{aligned}
$$

where $\varepsilon(x)=x \cdot \operatorname{erf}(x)+\exp \left(-x^{2}\right) / \sqrt{\pi}$ and $\sigma$ is the standard deviation of the diffusing profile given by

$$
\sigma=\sqrt{G_{D M A} \widetilde{D}}
$$

where $\widetilde{D}=4 \pi L \mathscr{D} /\left(Q_{e}+Q_{s h}\right)$ with $\mathscr{D}=k_{B} T C_{c}(K n) / 3 \pi \mu d_{p}$, and $G_{D M A}$ is a non-dimensional geometry factor defined as (Stolzenburg 1988)

$$
\begin{aligned}
G_{D M A} & =\frac{4(1+\beta)^{2}}{(1-\gamma)}\left\{\left[I_{\gamma}\left(\omega_{s}\right)+I_{\gamma}\left(\omega_{a}\right)\right]\right. \\
+ & {\left.\left[1+\frac{\beta(1-\delta)\left(1-\omega_{a}\right)}{\kappa_{a}(1-\gamma)}+\frac{\beta(1+\delta)\left(\omega_{s}-\gamma\right)}{\kappa_{s}(1-\gamma)}\right][2(1+\beta) \kappa]^{-2}\right\} }
\end{aligned}
$$

where $\gamma=\left(R_{1} / R_{2}\right)^{2}, \kappa=L R_{2} /\left(R_{2}^{2}-R_{1}^{2}\right), \kappa_{a}=\Delta z_{a} / L, \kappa_{s}=\Delta z_{s} / L$, and

$$
\begin{gathered}
I_{\gamma}(\omega)=A_{\gamma}^{2}(1-\gamma)^{-1}\left\{-\frac{1}{2} \omega^{2}[(1-\gamma) \ln (\omega)-(1-\omega) \ln (\gamma)]^{2}\right. \\
+\left[\frac{1}{2} \omega^{2}(1-\gamma)+\frac{1}{3} \omega^{3} \ln (\gamma)\right][(1-\gamma) \ln (\omega)-(1-\omega) \ln (\gamma)] \\
+\frac{1}{4}\left(1-\omega^{2}\right)(1-\gamma)^{2}+\frac{5}{18}\left(1-\omega^{3}\right)(1-\gamma) \ln (\gamma) \\
\left.+\frac{1}{12}\left(1-\omega^{4}\right) \ln ^{2}(\gamma)\right\}
\end{gathered}
$$

with

$$
A_{\gamma}=\left[-\frac{1}{2}(1+\gamma) \ln (\gamma)-(1-\gamma)\right]^{-1} \text {. }
$$


The values $\omega_{a}$ and $\omega_{s}$ are defined as

$$
\begin{aligned}
& F_{\gamma}\left(\omega_{a}\right)=\beta(1-\delta) / 2(1+\beta) \\
& 1-F_{\gamma}\left(\omega_{s}\right)=\beta(1+\delta) / 2(1+\beta)
\end{aligned}
$$

where

$$
F_{\gamma}(\omega)=-A_{\gamma}\left[\frac{\left(1-\omega^{2}\right)}{2(1-\gamma)} \ln (\gamma)+\omega \ln (\omega)+(1-\omega)\right], \quad \gamma \leq \omega \leq 1 .
$$

The shape of the non-diffusing transfer function is triangular with a mean electrical mobility corresponding to $Z_{p}^{*}$, a half-width of $\Delta Z_{p}$, and is piecewise continuous. The diffusing transfer function is a continuous function whose mean is skewed toward larger electrical mobilities. Figure 2 below illustrates the importance of the diffusing transfer function at small particle diameters and the convergence of the diffusing transfer function toward the ideal triangular transfer function at large particle diameters where diffusion effects at small. 


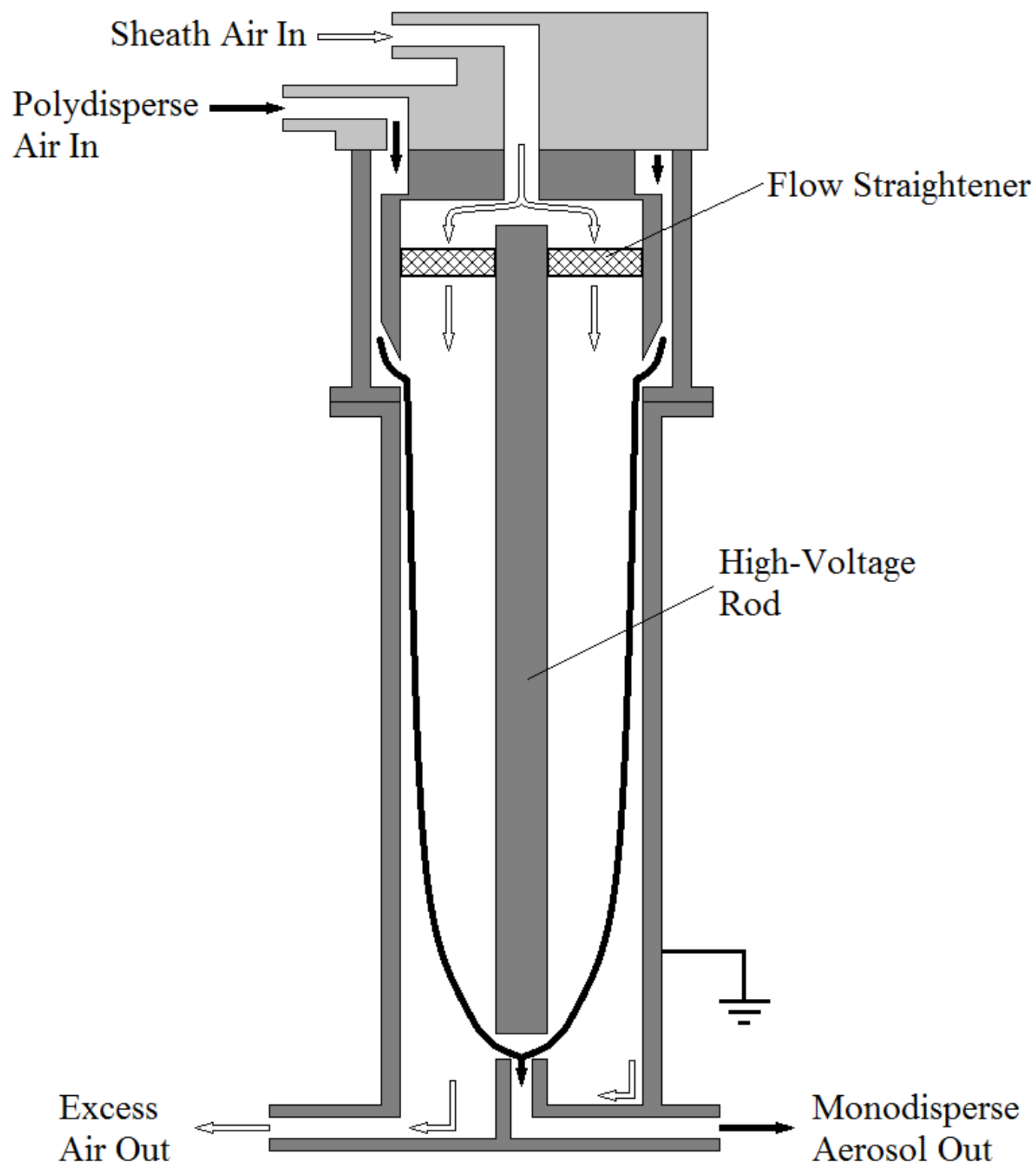

Figure 1. Schematic diagram of a typical cylindrical DMA showing trajectory of a charged particle with the characteristic electrical mobility, $Z_{p}^{*}$. 

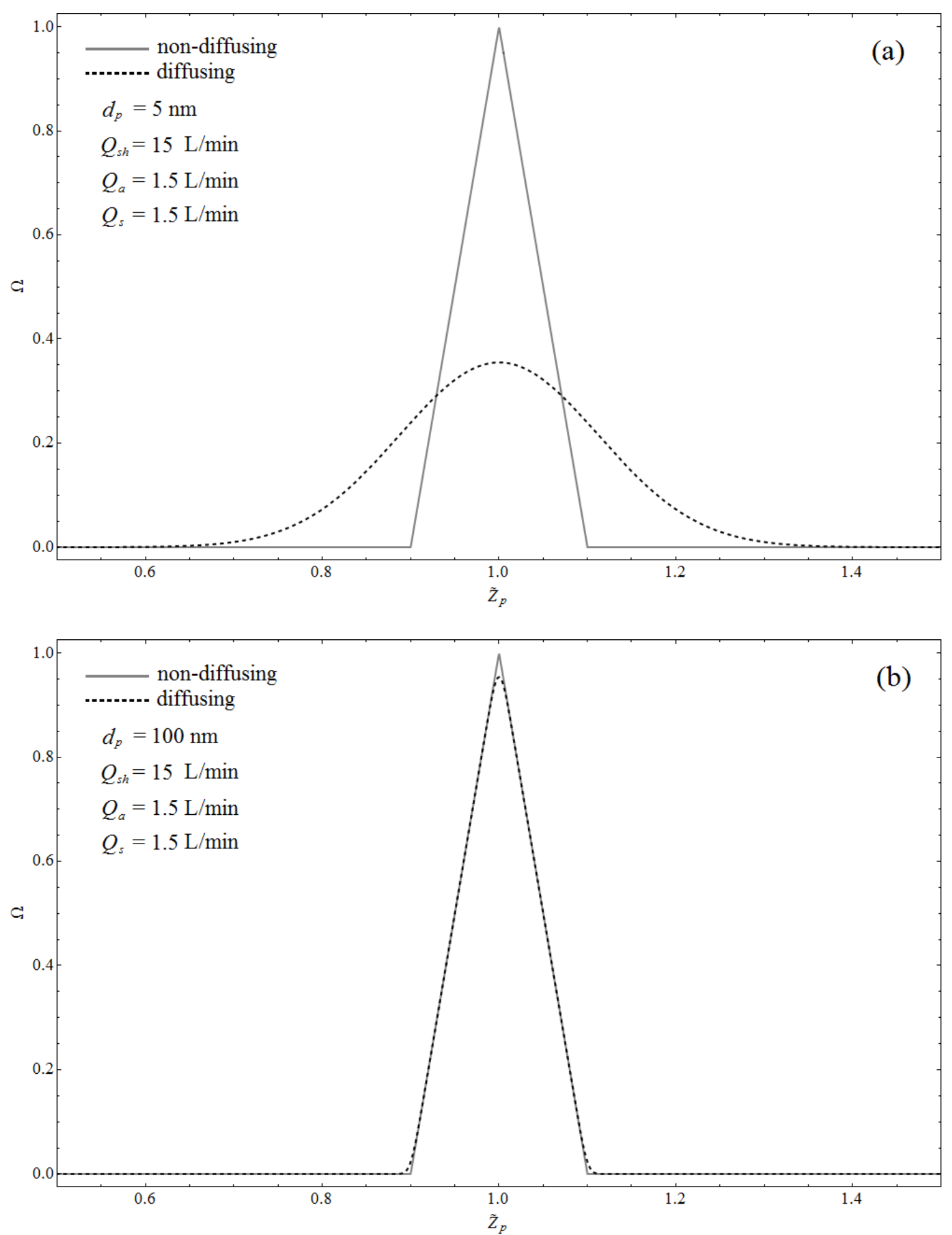

Figure 2. Comparison of non-diffusing $\left(\Omega_{n d}\right)$ and diffusing $\left(\Omega_{d}\right)$ transfer functions as a function of dimensionless electrical mobility, $\widetilde{Z}_{p}$, for a particle diameter of (a) $5 \mathrm{~nm}$ and (b) $100 \mathrm{~nm}$. 


\subsubsection{Scanning Mobility Particle Sizer (SMPS)}

The scanning mobility particle sizer (SMPS) is an instrument in common use today to obtain, quantitatively, the size distribution of submicron aerosol particles. The SMPS used in this study was the TSI 3936 Scanning Mobility Particle Sizer Spectrometer comprised of a radioactive charge neutralizer (TSI 3077A Aerosol Neutralizer), inertial impactor, DMA with support equipment for providing sheath flows and high voltage (TSI 3080 Electrostatic Classifier), and a condensation particle counter (TSI 3775 Condensation Particle Counter). During operation the sampled aerosol is first passed through a radioactive charge neutralizer before it is introduced into the DMA. Ionizing radiation from a sealed ${ }^{85} \mathrm{Kr}$ source attached to the inside the neutralizer cavity wall produces a high concentration of bipolar ions within the aerosol carrier gas. These ions undergo multiple collisions with the aerosol particles as they flow through the cavity. Through these collisions the aerosol particles will approach charge equilibrium, which has a very specific and well known charge distribution (Flagan 2001). Placing a known charge on the particles allows the particle size to be determined from electrical mobility measurements, which are otherwise dependent on both particle size and charge. After passing through the charge neutralizer, the aerosol is then introduced into the DMA and the voltage is scanned exponentially over time. During this time the classified aerosol, whose electrical mobility is a function of time, is supplied to the CPC which measures the particle concentration of the classified aerosol flow during the measurement interval. The aerosol size distribution, $n\left(d_{p}\right)$, is then determined, indirectly, through inversion of the set of Fredholm integrals (Wang and Flagan 1990)

$$
S_{j}=\sum_{n_{e}=1}^{\infty} \int_{0}^{\infty} n\left(d_{p}\right) s_{\text {count }}\left(d_{p}, n_{e}\right) \phi\left(d_{p}, n_{e}\right) \Omega\left[Z_{p}\left(d_{p}, n_{e}\right), t_{m}, t_{c}\right] \mathrm{d} d_{p}
$$


where $S_{j}$ is the CPC signal for the $j$ th time interval, $s_{\text {count }}\left(d_{p}, n_{e}\right)$ is the detector response to a particle of size $d_{p}$ and charge $n_{e}, \phi\left(d_{p}, n_{e}\right)$ is the probability for a particle size $d_{p}$ acquiring $n_{e}$ charges (i.e. equilibrium charge distribution), $t_{\mathrm{m}}$ is the measurement time, and $t_{c}$ is the counting or integration time. Inversion of Eq. 14 is quite involved and will not be covered here. For the purposes of this research, the inversion was handled within the TSI Aerosol Instrument Manager® (AIM) software supplied with the SMPS instrument, which provides the size distribution in real time and is in common use within industry. Because of the finite counting intervals during measurement, the SMPS results are presented in terms of a histogram with geometrically spaced bins, typically at a resolution of 64 bins/decade. To overcome difficulties in comparing measurements $(\mathrm{d} N)$ acquired at different resolutions, the measurements are also normalized with respect to the bin width $\left(\mathrm{d} N / \mathrm{d} \log d_{p}\right)$. The SMPS has a voltage range of 5-10,000 V, with the upper limit constrained by the electrical breakdown of the carrier gas. The resulting measureable size range is then dependent on the given combination of DMA geometry and flow rates. The AIM software also includes options for correcting the data for diffusion losses and multiply charged particles. In the latter case an impactor is used to define an upper cutoff diameter, within the measureable size range, so that multiple charge contributions can be subtracted from smaller diameters. Variations in temperature and pressure which effect fluid properties are also accounted for by the AIM software by interconnecting the CPC and DMA-2 using an RS-232 cable. The accuracy of the SMPS was confirmed by measuring monodisperse aerosols of known size and is described in Appendix A. 


\subsubsection{Tandem Differential Mobility Analyzer (TDMA)}

The TDMA is commonly used to measure the dependence of various aerosol processes (ex. condensation/evaporation rates, chemical reactions, etc.) on particle properties such as particle size and composition (see Park et al. (2008) for a review of TDMA measurements and applications). Additionally, the TDMA has also been shown to provide quantitative measurement of the aerosol size and charge distribution (Chadha et al. 2012; Kim et al. 2005; Maricq 2005; Simones et al. 2011; Simones et al. Submitted). When configured to measure size and charge distributions, the TDMA consists of two DMAs connected in series with a CPC. During operation the sampled aerosol is introduced into the first DMA (DMA-1) without prior charge neutralization and is therefore classified strictly by electrical mobility. With a constant voltage applied to DMA-1 the classified monomobile particles are then charge neutralized before entering the second DMA (DMA-2) where the voltage is scanned and classified particles are counted using the CPC to obtain the particle size distribution. By repeatedly stepping the voltage on DMA-1 and measuring the resulting size distribution, a size-mobility distribution is obtained. In this study a TSI 3080 Electrostatic Classifier was used for DMA-1 and a TSI 3936 Scanning Mobility Particle Sizer Spectrometer (TSI 3080 Electrostatic Classifier + TSI 3775 Condensation Particle Counter) was used for DMA-2 and the CPC. A LabVIEW® program was also created to automate and expedite the voltage stepping process via communication with DMA-1 through an RS-232 serial port and voltage measurement on DMA-2. Details on the LabVIEW® program and instrument interfacing can be found in Appendix B. 
To determine the size and charge distribution from the size-mobility distribution the data must be inverted through a Fredholm integral much like the SMPS measurements (Gysel et al. 2009; Rader and McMurry 1986; Stolzenburg and McMurry 2008), however in this work we took advantage of the SMPS inversion algorithm to simplify the TDMA inversion process. Using the SMPS results for the size distribution downstream of DMA-1, $n_{1}\left(d_{p}\right)$, the inversion process is reduced to determining the original distribution function supplied to DMA-1, $n_{0}\left(d_{p}, n_{e}\right)$, as depicted in Figure 3. These distribution functions are related by writing a particle balance for the rate of which particles of size $d_{p}$ enter and exit DMA-1

$$
n_{1}\left(d_{p}\right) Q_{s}=\sum_{n_{e}=1}^{n_{e} \Omega_{1}} \Omega_{1}\left(d_{p}, n_{e}, Z_{p}^{*}\right) n_{0}\left(d_{p}, n_{e}\right) Q_{a}
$$

Since the SMPS provides data for individual size bins, Eqn. 15 is discretized for each size bin of the SMPS, $d_{p, i}$,

$$
\begin{aligned}
& n_{1, j}\left(d_{p, 1}\right) Q_{s}=\sum_{n_{e}=1}^{n_{e}^{\max }} \Omega_{1}\left(d_{p, 1}, n_{e}, Z_{p, j}^{*}\right) n_{0}\left(d_{p, 1}, n_{e}\right) Q_{a} \\
& n_{1, j}\left(d_{p, 2}\right) Q_{s}=\sum_{n_{e}=1}^{n_{e}^{\max }} \Omega_{1}\left(d_{p, 2}, n_{e}, Z_{p, j}^{*}\right) n_{0}\left(d_{p, 2}, n_{e}\right) Q_{a}, \quad j=1,2,3, \ldots, J \\
& \vdots \\
& n_{1, j}\left(d_{p, I}\right) Q_{s}=\sum_{n_{e}=1}^{n_{e}^{\max }} \Omega_{1}\left(d_{p, I}, n_{e}, Z_{p, j}^{*}\right) n_{0}\left(d_{p, I}, n_{e}\right) Q_{a}
\end{aligned}
$$

where $I$ is the total number of size bins and Eqn. 16 is repeated over all $J$ mobility settings on DMA-1. To isolate the dependence of particle size we consider the set of all mobility measurements, $J$, for a single size bin, $i$, 


$$
\begin{aligned}
& n_{1,1}\left(d_{p, i}\right) Q_{s}=\sum_{n_{e}=1}^{n_{e}^{\max }} \Omega_{1}\left(d_{p, i}, n_{e}, Z_{p, 1}^{*}\right) n_{0}\left(d_{p, i}, n_{e}\right) Q_{a} \\
& n_{1,2}\left(d_{p, i}\right) Q_{s}=\sum_{n_{e}=1}^{n_{e}^{\max }} \Omega_{1}\left(d_{p, i}, n_{e}, Z_{p, 2}^{*}\right) n_{0}\left(d_{p, i}, n_{e}\right) Q_{a}, \quad i=1,2,3, \ldots, I . \\
& \vdots \\
& n_{1, J}\left(d_{p, i}\right) Q_{s}=\sum_{n_{e}=1}^{n_{e}^{\max }} \Omega_{1}\left(d_{p, i}, n_{e}, Z_{p, J}^{*}\right) n_{0}\left(d_{p, i}, n_{e}\right) Q_{a}
\end{aligned}
$$

Eqn. 17 can then be written in matrix form

$$
\underbrace{\left[\begin{array}{c}
n_{1,1}\left(d_{p, i}\right) \\
n_{1,2}\left(d_{p, i}\right) \\
\vdots \\
n_{1, J}\left(d_{p, i}\right)
\end{array}\right]}_{b}=\underbrace{\left[\begin{array}{cccc}
\Omega_{1}\left(d_{p, i}, 1, Z_{p, 1}^{*}\right) & \Omega_{1}\left(d_{p, i}, 2, Z_{p, 1}^{*}\right) & \cdots & \Omega_{1}\left(d_{p, i}, n_{e}^{\max }, Z_{p, 1}^{*}\right) \\
\Omega_{1}\left(d_{p, i}, 1, Z_{p, 2}^{*}\right) & \Omega_{1}\left(d_{p, i}, 2, Z_{p, 2}^{*}\right) & \cdots & \Omega_{1}\left(d_{p, i}, n_{e}^{\max }, Z_{p, 2}^{*}\right) \\
\vdots & \vdots & & \vdots \\
\Omega_{1}\left(d_{p, i}, 1, Z_{p, J}^{*}\right) & \Omega_{1}\left(d_{p, i}, 2, Z_{p, J}^{*}\right) & \cdots & \Omega_{1}\left(d_{p, i}, n_{e}^{\max }, Z_{p, J}^{*}\right)
\end{array}\right]}_{A} \underbrace{\left[\begin{array}{c}
n_{0}\left(d_{p, i}, 1\right) \\
n_{0}\left(d_{p, i}, 2\right) \\
\vdots \\
n_{0}\left(d_{p, i}, n_{e}^{\max }\right)
\end{array}\right]}_{x}
$$

and $n_{0}\left(d_{p, i}, n_{e}\right)$ can then be determined using the method of least squares. Here a weighted least squares solution is introduced to account for unequal counting errors (Strang 1980)

$$
W b=W A x
$$

where, for uncorrelated errors,

$$
W=\left[\begin{array}{cccc}
w_{1} & 0 & 0 & \cdots \\
0 & w_{2} & 0 & \cdots \\
0 & 0 & w_{3} & \cdots \\
\vdots & \vdots & \vdots &
\end{array}\right]
$$

and the weights, $w_{j}$, are given by the count data of the SMPS: $w_{j}=b_{j}$. Uncertainty in the values for $n_{0}\left(d_{p, i}, n_{e, k}\right)$ can then be estimated using (Wolberg 2006)

$$
\sigma n_{0}\left(d_{p, i}, n_{e, k}\right)=\left(\frac{S_{i}}{J-n_{e}^{\max }} C_{i, k k}^{-1}\right)^{\frac{1}{2}}
$$

where $S_{i}$ is the objective function defined as 


$$
S_{i}=\sum_{j=1}^{J} w_{i}\left\{n_{1, j}\left(d_{p, i}\right)-\sum_{n_{e}=1}^{n_{e}^{\max }}\left[\Omega_{1}\left(d_{p, i}, n_{e}, Z_{p, j}^{*}\right) n_{0}\left(d_{p, i}, n_{e}\right)\right]\right\}^{2}
$$

and the terms $C_{j k}$ are defined as

$$
C_{i, k l}=\sum_{j=1}^{J} w_{j} \Omega_{1}\left(d_{p, i}, n_{e, k}, Z_{p, j}^{*}\right) \Omega_{1}\left(d_{p, i}, n_{e, l}, Z_{p, j}^{*}\right) .
$$

Accuracy of this TDMA inversion method was confirmed by performing size and charge distribution measurements on a charged neutralized aerosol and comparing the results against theoretical equilibrium charge distributions (see Appendix $\mathrm{C}$ for details). 


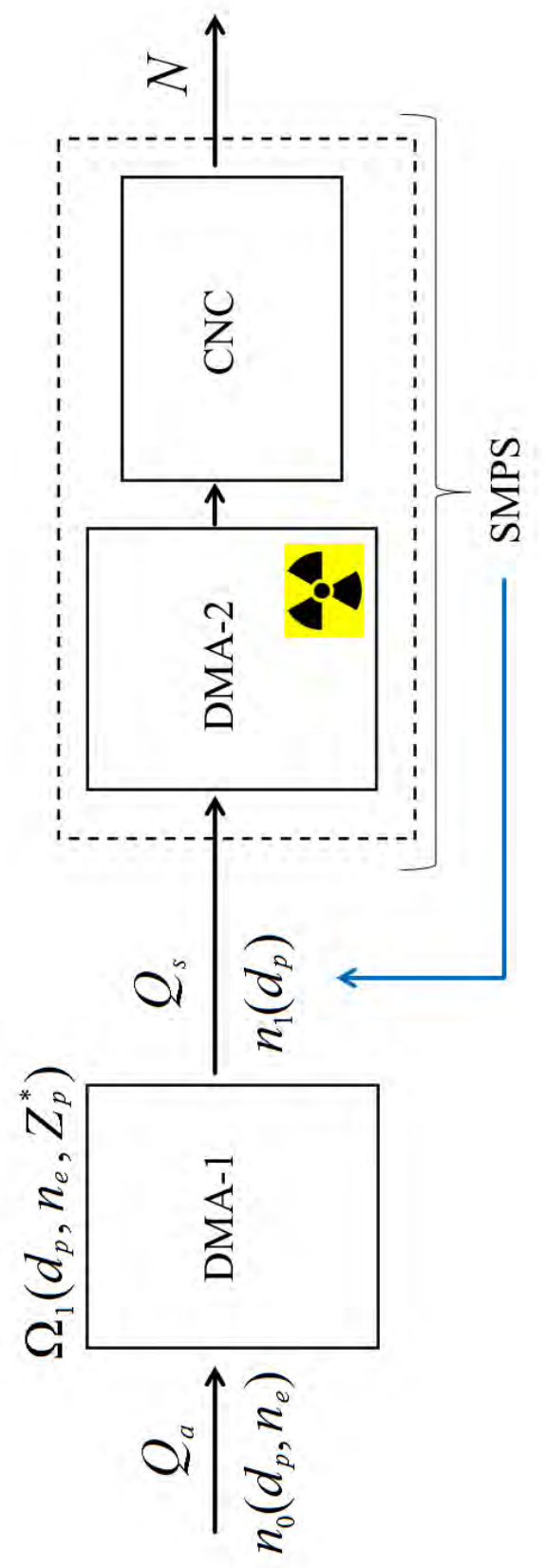

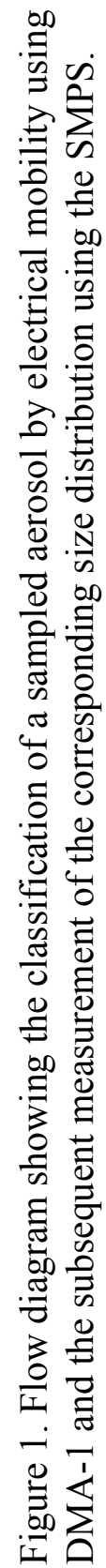




\subsection{Aerosol Generation}

During this study aerosols were generated using a spark generator, namely a PALAS Defined Nano Particle Generator model DNP-3000 for carbon aerosol and the model GFG-1000 for silver aerosol. Particles are produced through a capacitive discharge process between two electrodes positioned within an isolated gas chamber. A high potential is applied across the electrode gap causing electrical breakdown of the present gas and a momentary flow of electrical current. The resulting spark creates an isolated high temperature region on each electrode, ablating the electrode surface. An inert carrier gas $\left(\mathrm{N}_{2}\right)$ is directed into the electrode gap which rapidly condenses any vapor present to form nanosized primary particles, and clears the electrode gap of ions and ablated material for the next discharge cycle. As the primary particles exit the gas chamber they continue to collide with other particles as well as ions forming charged agglomerates, and the resulting test aerosol. Adjustment of operating parameters such as spark frequency, spark energy, current, and gas flow rate allow the user to manipulate the quantity of ablated material and the rate of particle production which control the output particle distribution (i.e. mean particle size, standard deviation, total concentration, etc.). Previous analysis using transmission electron microscopy (TEM), has shown that carbon nanoparticles produced with the spark generator form complex chain agglomerates (Figure 4a), while silver nanoparticles produced with the spark generator form single particles that are nearly spherical in shape (Figure 4b) (Boddu et al. 2011; Simones et al. 2011). 

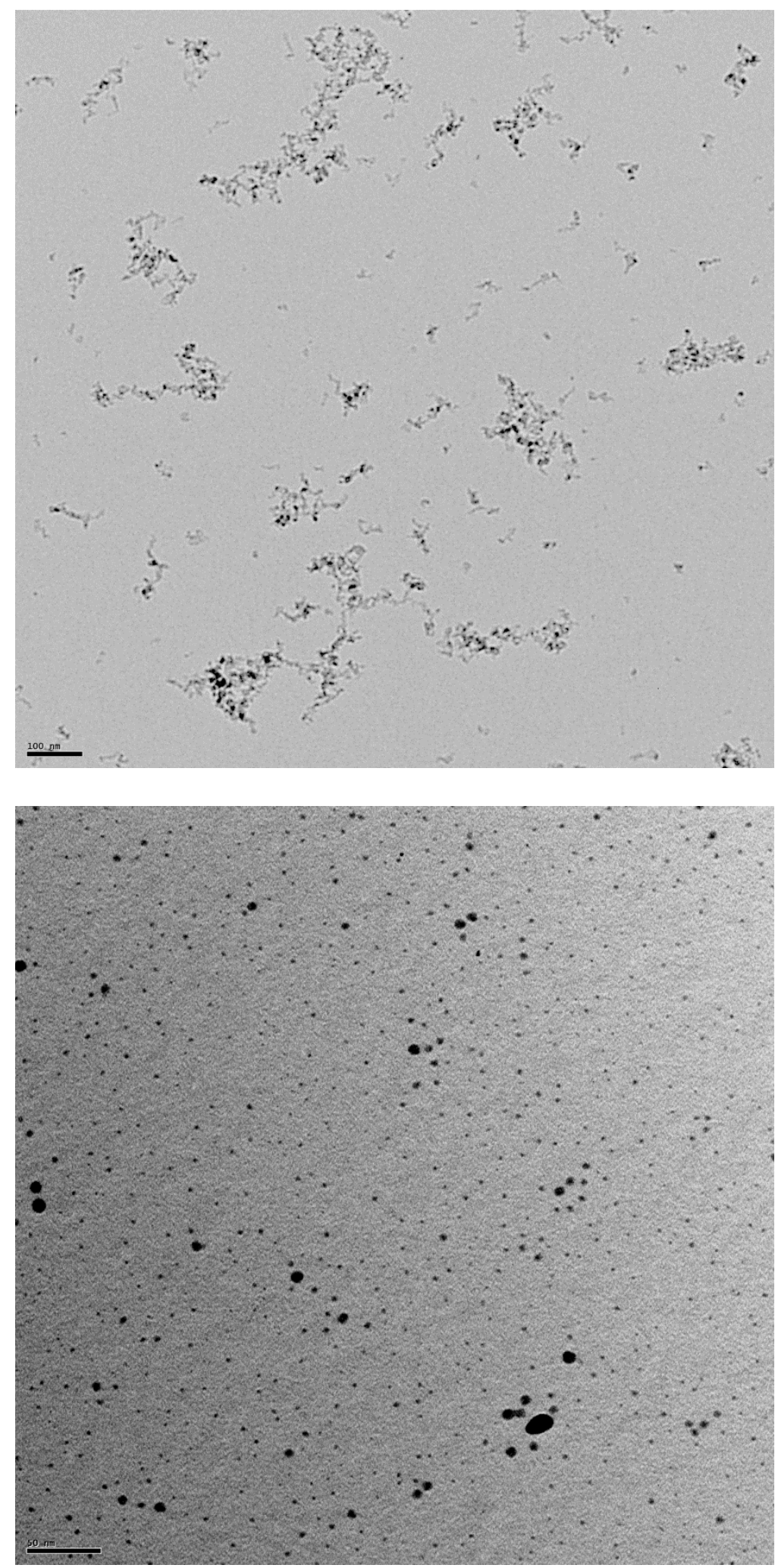

Figure 2. TEM images of spark generated (a) graphite (100 nm scale) and (b) silver (50 nm scale). Courtesy of Dr. Veera Rajesh Gutti, NSEI. 


\subsection{Coagulation Chamber}

In pursuing measurements of aerosol coagulation using the TDMA, an open flow coagulation chamber, in contrast to a closed chamber, is necessary because a single TDMA measurement could take several hours, during which a steady aerosol sample must be supplied. A closed coagulation chamber would not be able to provide a constant source over this length of time without considerable changes in the size and charge distribution, not to mention the change in volume required for the amount of sample withdrawn during measurement. The open flow system, shown in Figure 5, consists of a cylindrical tube in which aerosol is continuously flowing and allows a constant source of aerosol to be sampled as a function of axial distance along the tube length (i.e. residence time). An important aspect of the open flow design is that the concentration boundary layer develops slowly, over multiple chamber lengths, which provides a centerline aerosol concentration that is independent of wall effects (Friedlander 2000). The main design parameters for the open flow chamber include flow rate, tube diameter, tube length, and particle size range. These parameters determine all other design criteria including Reynolds number for laminar flow conditions and development of the hydrodynamic boundary layer (entrance length), Péclet number for diffusion losses and development of the concentration boundary layer, particle residence time within chamber, gas velocity profile, and sampling probe inlet diameter for sampling conditions and aspiration efficiency. Since many of the above design criteria are interdependent, a parametric analysis was performed to aid in the selection of appropriate design parameters. First, an estimate of the time scale required for measurable coagulation was determined using the analytical results for simple monodisperse coagulation (Fuchs 1964) 


$$
N(t)=\frac{N_{0}}{1+N_{0} K_{0} \beta t}
$$

where $N(t)$ is the aerosol concentration as a function of time $t, N_{0}$ is the initial

concentration, $K_{0}$ is the limiting coagulation coefficient for coarse particles $\left(d_{p}>100 \mathrm{~nm}\right)$ defined as

$$
K_{0}=4 \pi d_{p} \mathscr{D}=\frac{4 k_{B} T C_{c}(K n)}{3 \mu}
$$

and $\beta$ is a correction factor for ultrafine particles. Since all particle sampling will occur at the chamber centerline, the coagulation time indicated by Eqn. 24 was used as the target value for centerline particle residence time, $t_{\text {res }}$. The centerline residence time was used in selection of the chamber length, $L_{c}$, and diameter, $D$, based on the laminar flow criterion $\left(R e_{D}<2100\right)$ and a selection of volumetric flow rate within the operating range of the spark generator. For laminar flow the fluid velocity at the chamber centerline is given by the Hagan-Poiseuille equation (Bird et al. 2007)

$$
u_{z, \max }=\frac{8 Q}{\pi D^{2}}=\frac{L_{c}}{t_{\text {res }}}
$$

Additionally, the hydrodynamic entry length was estimated by (Incropera et al. 2007)

$$
L_{h} \approx 0.05 D \operatorname{Re}_{D}
$$

and was added to $L_{c}$ to determine the overall tube length required. The diffusive entry length given by (Incropera et al. 2007)

$$
L_{\mathscr{D}} \approx 0.05 D P e_{D}=0.05 D R e_{D} S c
$$

was also used as a check to ensure that the concentration boundary layer remained small relative to the hydrodynamic boundary layer over the length of the test section. The chamber tube diameter was selected from standard pipe sizes with consideration to wall thickness to accommodate sampling ports and fittings. 
As mentioned earlier, aerosol sampling will be conducted along the length of the coagulation chamber centerline. A series of sampling ports have been designed into the length of the coagulation chamber such that the sampling probe can be moved sequentially from the upstream to downstream side. Sampling ports not in use, especially those upstream of the sampling probe, need to be closed appropriately to avoid flow disturbances which will effect coagulation. With this in mind a port cover or 'cap' has been designed which is able to seal the sampling port while maintaining a smooth, continuous profile on the inside of the chamber. A sampling probe cap has also been designed to replace the port cap during sampling and allows the sampling probe to be adjusted vertically for alignment with the chamber centerline. These caps have been constructed of ABS thermoplastic using the rapid prototyping resources available at the University of Missouri College of Engineering (see Appendix D for a description of the port cap and sampling probe cap). The sampling ports were spaced along the length of the chamber in a geometric series since the rate of change of the number concentration is expected to behave similarly to the power-law decay given in Eqn. 24. A sharp-edged sampling probe was used which contains a 90 degree bend so that it is aligned parallel to the gas flow for isoaxial sampling. The probe inlet extends upstream from the port location to minimize flow disturbances from the probe assembly. The sampling probe diameter and flow rate were selected to achieve a high aspiration efficiency considering both inertial and gravitational settling. Correlations for isoaxial aspiration efficiency can be separated into three regimes depending on the free gas stream velocity (Brockmann 2001; Vincent 2007). For sampling from a flowing gas stream the aspiration efficiency, $\eta_{a s p}$, is given by 


$$
\eta_{a s p}=1+\left(\frac{U_{0}}{U}-1\right)\left(1-\frac{1}{1+k S t k_{d, U_{0}}}\right)
$$

where $k=2+0.617\left(U / U_{0}\right), U_{0}$ is the free stream velocity, $U$ is the sampling velocity, and $S t k_{d, U_{0}}$ is the Stokes number evaluated using the sampling probe inlet diameter, $d$, and the free stream velocity. For sampling from calm air $\left(U_{0}=0\right)$ the aspiration efficiency is given by

$$
\eta_{a s p, c a l m a i r}=\exp \left\lfloor-\frac{4 S t k_{d}^{1+\sqrt{\frac{V_{s}}{U}}}}{1+2 S t k_{d, U}}\right\rfloor
$$

where $V_{t s}=\tau g$ is the particle terminal settling velocity and $\tau$ is the particle relaxation time given by

$$
\tau=\rho_{p} d_{p}^{2} C_{c}(K n) / 18 \mu
$$

For slowly moving air the aspiration efficiency is represented as a combination of aspiration efficiencies for moving and calm air

$$
\eta_{\text {asp,tot }}=f_{\text {moving }} \eta_{\text {asp }}(1+\delta)^{1 / 2}+f_{\text {calm }} \eta_{\text {asp calmair }}
$$

where

$$
\begin{gathered}
\delta=\left(V_{t s} / U_{0}\right)^{2}, \\
f_{\text {moving }}=\exp \left(-V_{t s} / U_{0}\right), \\
f_{\text {calm }}=1-\exp \left(-V_{t s} / U_{0}\right),
\end{gathered}
$$

At small free stream velocities the sampling conditions may be considered 'calm' if

$$
U_{0}<\frac{0.1 U}{S t k_{d, U_{0}}^{2 / 3}}
$$


Under calm conditions representative sampling $\left(\eta_{\text {asp,calmair }} \approx 1\right)$ can then be achieved if the following criteria are met:

$$
S t k_{d, U} \leq 0.016
$$

and

$$
\frac{V_{t s}}{U} \leq 0.04
$$

A dilution device was also installed externally from the chamber immediately following the sampling probe to minimize further coagulation between the coagulation chamber and the measuring instruments. The dilution device is similar to the ejector pump described by Maricq (2005), where the aerosol sampling tube is aligned coaxially within a larger tube as shown in Figure 6. The aerosol is introduced at the centerline of the larger tube while a controlled volume of clean dilution gas (i.e. $\mathrm{N}_{2}$ ) is introduced through a tee fitting and flows around the outside of the sample tube as it enters the larger tube. This method minimizes aerosol losses due to inertial and diffusional deposition within the dilution device.

Additional consideration was given to a diffuser for expanding the flow from the transport tubing diameter of the spark generator to the coagulation chamber diameter, and to a nozzle for the flow exiting the chamber. The flow conditions within the diffuser are inherently unstable and require careful design to minimize flow recirculation from rapid expansion while minimizing the overall length of the diffuser. Typically for a simple straight wall diffuser, a maximum diffuser wall separation angle of $15^{\circ}$ is used as a general rule of thumb to prevent boundary layer separation. In this case, the large expansion ratio requires the simple diffuser to have a substantial overall length. To decrease the diffuser length while preventing boundary layer separation, deflectors were 
incorporated into the diffuser design as suggested by Cheng (1992). COMSOL simulations of the coagulation chamber fitted with this type of diffuser have shown that flow separation does not occur and that the flow remains laminar throughout the sample region. Further details of the diffuser and nozzle designs including COMSOL results and engineering drawings are provided in Appendix E. The overall length of the coagulation chamber including the diffuser and nozzle was restricted to 3 meters based on the lab space available. Details of the parametric analysis are provided within the Mathematica program in Appendix F and a summary of the design criteria and the corresponding design parameters chosen are summarized in Table 2. 
Table 2. Summary of design criteria and selected parameters

Design Criteria

Particle size range $(\mathrm{nm})$

Spark generator outlet tube inside diameter $(\mathrm{mm})$

Aerosol flow rate $(1 / \mathrm{min})$

Aerosol sampling flow rate $(1 / \mathrm{min})$

Maximum overall length (m)

Fluid residence time at centerline (s)

Selected Parameters

Chamber inside diameter (NPS $4 \mathrm{SCH} 80)(\mathrm{mm})$

Chamber Length, $L_{c}(\mathrm{~m})$

Sampling probe inlet diameter, $d(\mathrm{~mm})$

Fluid velocity at centerline $(\mathrm{m} / \mathrm{s})$

Fluid residence time at centerline (s)

$R e_{D}$ inside chamber

Hydrodynamic entrance length, $L_{h}(\mathrm{~m})^{\dagger}$

Diffusion entrance length for $\mathrm{d}_{\mathrm{p}}=5 \mathrm{~nm}, \mathrm{~L}_{\mathscr{D}}(\mathrm{m})^{\dagger}$

Diffusion entrance length for $d_{p}=700 \mathrm{~nm}, L_{\mathscr{D}}(\mathrm{m})^{\dagger}$

Diffuser wall separation angle $\left({ }^{\circ}\right)$

Nozzle wall separation angle $\left({ }^{\circ}\right)$

Diffuser length, $L_{d}(\mathrm{~m})$

Nozzle length (m)

Calm air condition for $d_{p}=5 \mathrm{~nm}(\mathrm{~m} / \mathrm{s})$ (Eqn. 36)

Calm air condition for $d_{p}=700 \mathrm{~nm}(\mathrm{~m} / \mathrm{s})$ (Eqn. 36)

Calm air inertial criteria for $d_{p}=5 \mathrm{~nm}$ (Eqn. 37)

Calm air inertial criteria for $d_{p}=700 \mathrm{~nm}$ (Eqn. 37)

Calm air settling velocity criteria for $d_{p}=5 \mathrm{~nm}$ (Eqn. 38)

Calm air settling velocity criteria for $d_{p}=700 \mathrm{~nm}$ (Eqn. 38)

$$
\begin{gathered}
5-700 \\
8 \\
1.5 \\
0.15 \\
3 \\
>300
\end{gathered}
$$

97

2.8

1.75

$6.79 \times 10^{-3}$

411

21.8

0.11

7.4

$3.8 \times 10^{4}$

45

45

0.11

0.11

$6.06 \times 10^{6}$ (true)

$1.69 \times 10^{12}$ (true)

0.0115 (true)

$2.18 \times 10^{-5}$ (true)

$1.84 \times 10^{-4}$ (true)

$3.49 \times 10^{-7}$ (true)

${ }^{\dagger}$ Assuming uniform flow at entrance 
(a)

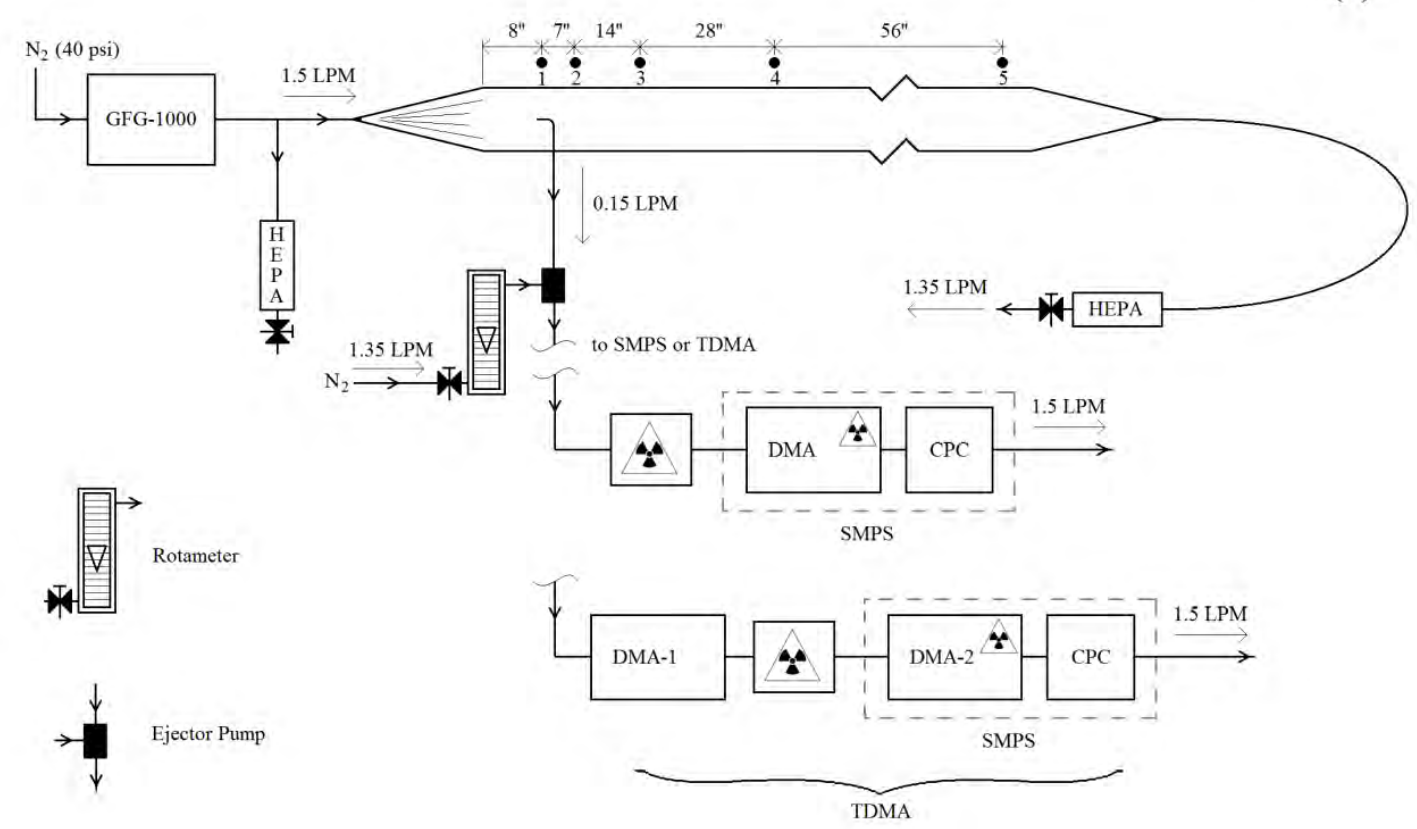

(b)

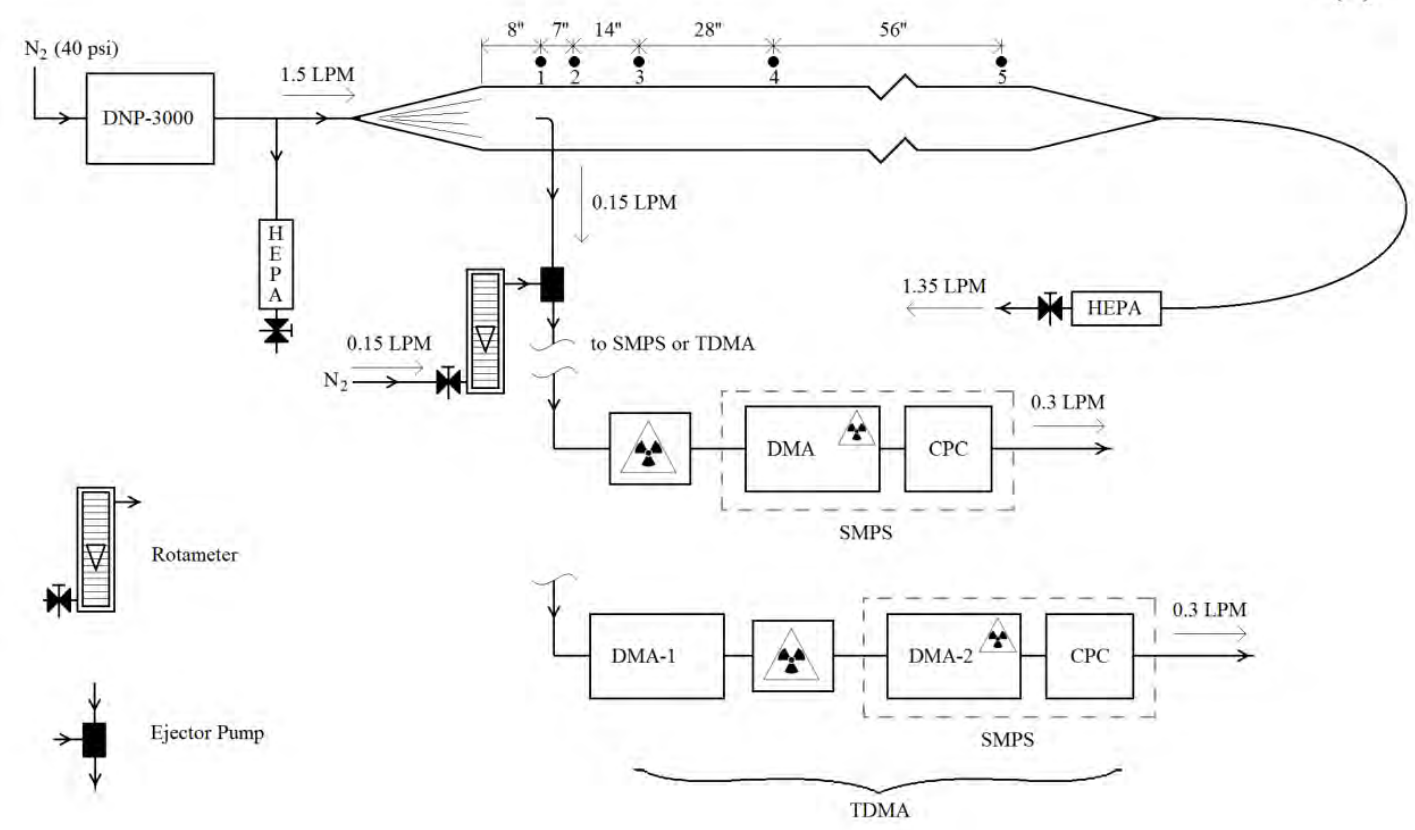

Figure 5. Schematic of the experimental setup for (a) silver and (b) carbon aerosol size and charge distribution measurements. Aerosol is sequentially sampled from each of the five sampling ports along the length of the chamber. During measurement the SMPS and TDMA are alternately connected to the ejector pump. 


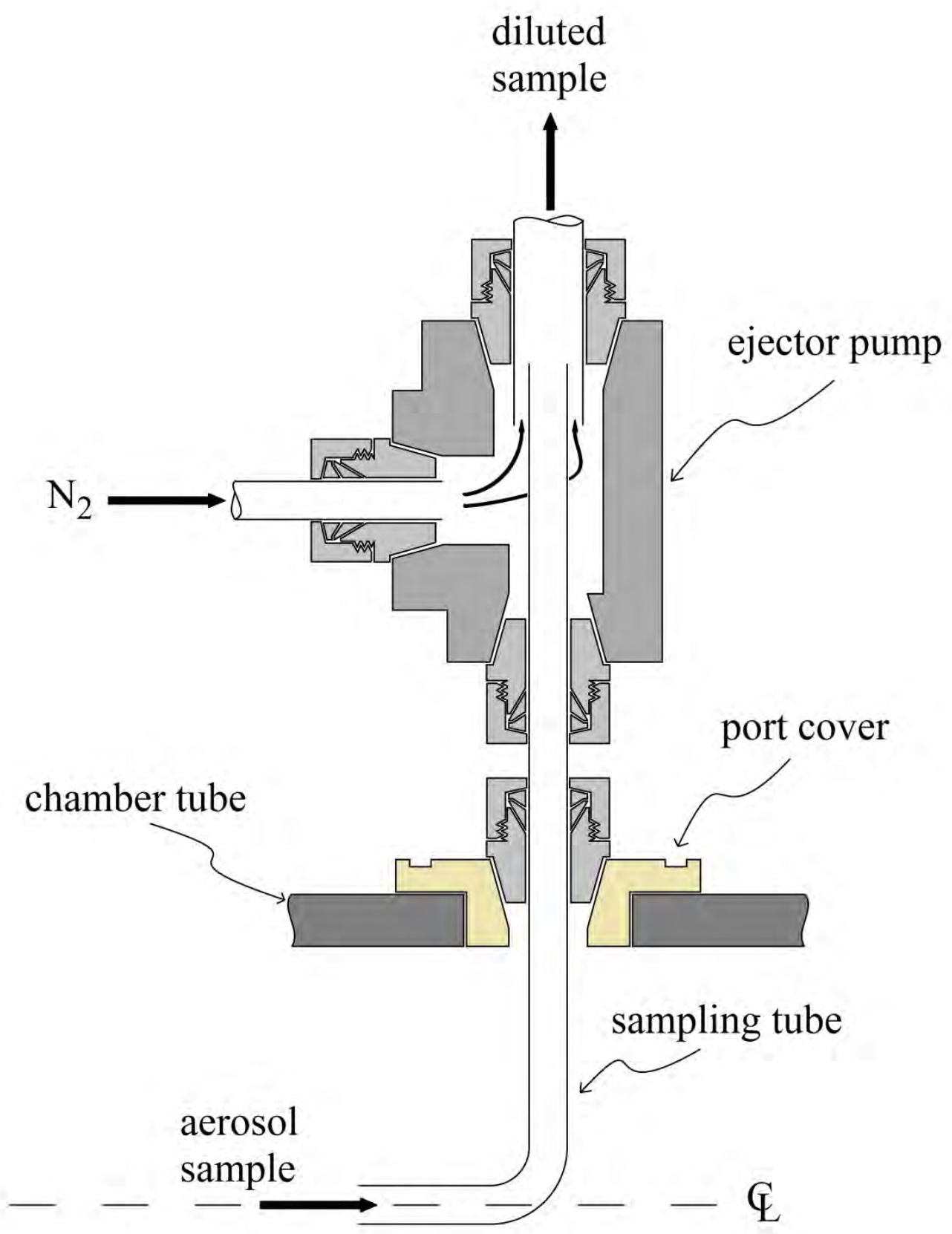

Figure 6. Schematic cross sectional view of aerosol sampling probe and dilution device (ejector pump). The sample probe is aligned coaxially with the centerline of the coagulation chamber and the aerosol sample is immediately diluted using an ejector pump located on the outside of the chamber wall. 


\section{Chapter 3}

\section{METHOD}

\subsection{Aerosol Measurements}

To begin coagulation measurements the spark generator, DMA-1, and SMPS were turned on and allowed to warm up for one hour with the CPC and sheath flows set. The CPC and sheath flow rates were predetermined with consideration to the expected size range of particles produced by the spark generator and the shift in particle size due to coagulation. The ratio of $\mathrm{CPC}$ to sheath flow rates was maintained at a value of ten throughout this study as recommended by the manufacturer for good resolution with the DMAs. Calibration of the chamber flow rate was carried out by first setting the $\mathrm{N}_{2}$ gas flow rate on the spark generator and installing the sampling probe in port \#1. Since the spark generator operates at a higher flow rate than desired for the coagulation chamber, excess flow was exhausted through a HEPA filter and needle valve. The flow rate through the chamber was calibrated using a bubble flow meter (Supelco Optiflow 650 Digital Flowmeter) installed in line at the diffuser inlet while using needle valves to balance the flow across the chamber. The dilution flow rate was then set by adjusting the rotameter and the value was confirmed using the bubble flow meter. After the warm up period particle generation was initiated on the spark generator and the system was given fifteen minutes to reach equilibrium. The SMPS was then used to obtain the initial size distribution which also assisted in the selection of voltage steps for DMA-1 during TDMA measurement. Here each voltage step, $V_{i}$, was defined such that the characteristic 
electrical mobility for a singly charged particle was centered on each size bin, $d_{p, i}$, of the SMPS within the size range selected.

$$
Z_{p}^{*}\left(V_{i}\right)=Z_{p}\left(d_{p, i}, 1\right) \rightarrow V_{i}, \quad i=1,2,3 \ldots I
$$

To prepare the TDMA measurements the SMPS was setup to perform I consecutive scans, each separated by a 30 second time delay to account for the finite time required for particles to traverse the distance between DMA-1 and DMA-2. The list of voltages determined from Eqn. 39 were entered into the LabVIEW program and the SMPS scan sequence was then initiated. Gas temperature and pressure values for DMA-1 were also recorded for each individual scan for corrections in viscosity and mean free path values during post-processing, while the AIM software accounted for these effects in DMA-2. Upon completion of the SMPS scan sequence, the DMA-1 voltage polarity was changed by exchanging the high voltage module installed and the SMPS scan sequence was repeated. After measurements at each polarity the SMPS was reconnected and the size distribution was again measured to check for changes over the time of the scan sequences. The sampling probe was then moved to sampling port \#2 and the process was repeated until measurements at all five sampling ports were completed. Table 3 below summarizes the instrument settings used during experimental measurements. 
Table 3. Summary of instrument settings used for both silver and carbon aerosol measurements

\begin{tabular}{lcc}
\hline Parameter & $\mathrm{Ag}$ & $\mathrm{C}$ \\
\hline Aerosol Generator & GFG-1000 & DNP-3000 \\
$\mathrm{N}_{2}$ flow rate $(1 / \mathrm{min})$ & $1.5(1.25$ bar $)$ & 3.0 \\
$\quad$ Spark frequency $(\mathrm{Hz})$ & 50 & \\
Spark energy & & "medium" \\
Current $(\mathrm{mA})$ & $5.94-224.7$ & $14.7-697.8$ \\
Particle size range $(\mathrm{nm})$ & 1.5 & 0.3 \\
CPC flow rate $(1 / \mathrm{min})$ & 1.35 & 0.15 \\
Dilution flow rate $(1 / \mathrm{min})$ & 0.15 & 0.15 \\
Sample flow rate $(1 / \mathrm{min})$ & 15.0 & 3.0 \\
DMA-1 sheath flow rate $(1 / \mathrm{min})$ & 15.0 & 3.0 \\
DMA-2 sheath flow rate $(1 / \mathrm{min})$ & - & 0.0457 \\
Impactor nozzle diameter $(\mathrm{cm})$ & - & 612 \\
Cutoff diameter, D50 $(\mathrm{nm})$ & & \\
\hline \hline
\end{tabular}

\subsection{Data Analysis}

The raw data from the TDMA provides the mobility-size distribution of the sampled aerosol while the SMPS measurement provides the size distribution over all charge levels (positive, negative, and neutral). Analysis of the TDMA raw data was carried out in the Mathematica program shown in Appendix G. Analysis consisted of correcting data for transport losses in transport tubing, DMA-1 (the SMPS automatically accounts for losses in DMA-2), the charge neutralizer, and the sampling probe; correcting data for multiply charged particles which were miscounted as singly charged; and performing the inversion process described in Section 2.1.3 to obtain the size and charge distribution. Diffusion losses in transport tubing were estimated using the model of Gormley and Kennedy (1949) for the transport efficiency in laminar tube flow as reviewed by both Brockmann (2001) and Cheng (2001), 


$$
\eta_{\text {tube, diff }}=\left\{\begin{array}{cc}
0.81905 \exp (-3.6568 \xi)+0.09753 \exp (-22.305 \xi) & \xi>0.02 \\
\quad+0.0325 \exp (-56.961 \xi)+0.01544 \exp (-107.62 \xi) & \\
1.0-2.5638 \xi^{2 / 3}+1.2 \xi+0.1767 \xi^{4 / 3} & \xi \leq 0.02
\end{array}\right.
$$

where $\xi=\pi \mathscr{D} L / Q$. Diffusion losses in DMA-1 were estimated by the empirical results provided by Karlsson and Martinsson (2003),

$$
\eta_{D M A}=\lambda_{E} \cdot \lambda_{D}
$$

with

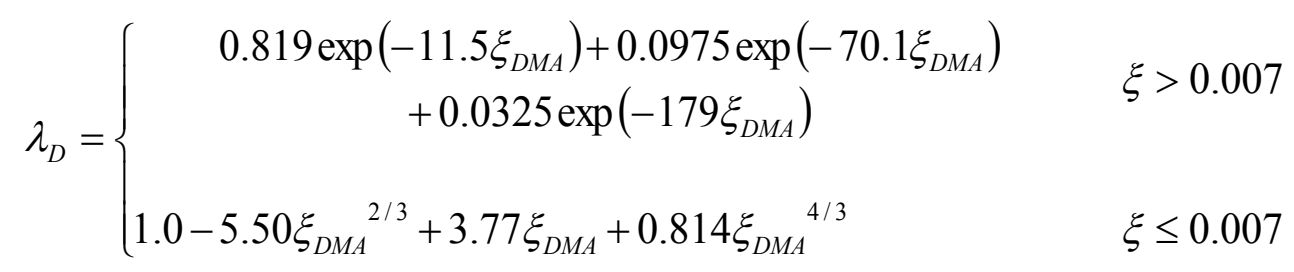

and $\xi_{D M A}=\mathscr{D} L_{e f f} / Q$. By fitting the model to experimental data Karlsson and Martinsson found that for the TSI model 3081 'long' DMA $L_{e f f}=7.1 \mathrm{~m}$ and $\lambda_{E}=0.98$. The transmission efficiency of the charge neutralizer (TSI 3077) was estimated by fitting Eqn. 40 to data provided by Covert et al. (1997). The fitting parameter, $L$, was found to be $L_{3077}=0.58 \mathrm{~m}$ for a sample flow rate of $1.5 \mathrm{LPM}$ and approximately $L_{3077}=0.2 \mathrm{~m}$ for a sample flow rate of 0.3 LPM. Particle sampling efficiency was estimated using the relations for aspiration efficiency reviewed in Section 2.3. For the particular system parameters used in this experiment the criteria for representative sampling $\left(\eta_{\text {asp,calmair }} \approx 1\right)$ given in Eqns. 37 and Eqn. 38 were maintained. Corrections for multiply charged particles in the TDMA data were made following the arguments described by Kim et al. (2005), where assumptions are made based on knowledge of the equilibrium charge distribution and the largest particle diameter in the sample defined by the impactor. Additionally, since the transfer function may vary over the size interval, an average value 
for the transfer function over each size interval was determined for use as the representative value in the data inversion process. This was done using the normal definition for the average value of a function

$$
\bar{\Omega}\left(d_{p, i}, n_{e}, Z_{p, j}^{*}\right)=\frac{1}{d_{p, i}^{H i g h}-d_{p, i}^{L o w}} \int_{d_{p, i}^{L, w}}^{d_{p, i}^{\text {Liph }}} \Omega\left(d_{p}, n_{e}, Z_{p, j}^{*}\right) d\left(d_{p}\right)
$$

where integration is carried out over the $i$ th size interval with lower and upper limits of

$d_{p, i}^{\text {Low }}$ and $d_{p, i}^{\text {High }}$, respectively. The size and charge distribution was then computed using the least squares technique outlined in Section 2.1.3.

\subsection{Numerical Simulations of Aerosol Coagulation}

\subsubsection{Sectional Method}

The sectional method implemented here follows the scheme outlined by Vemury et al. (1997) for the coagulation of bipolar aerosols:

$$
\begin{aligned}
& \frac{d n_{p_{e}}^{i}}{d t}=\sum_{q_{e}=-\infty}^{\infty} \sum_{j}^{i-2} 2^{j-i+1} \beta_{p_{e}-q_{e}, q_{e}}^{i-1, j} n_{p_{e}-q_{e}, q_{e}}^{i-1} n_{q_{e}}^{j}+\frac{1}{2} \sum_{q_{e}=-\infty}^{\infty} \beta_{p_{e}-q_{e}, q_{e}}^{i-1, i-1} n_{p_{e}-q_{e}}^{i-1} n_{q_{e}}^{i-1} \\
& \quad-\sum_{q_{e}=-\infty}^{\infty} \sum_{j=1}^{i-1} 2^{j-i} \beta_{p_{e}, q_{e}}^{i, j} n_{p_{e}}^{i} n_{q_{e}}^{j}-\sum_{q_{e}=-\infty}^{\infty} \sum_{j=1}^{\infty} \beta_{p_{e}, q_{e}}^{i, j} n_{p_{e}}^{i} n_{q_{e}}^{j}-B(i) \frac{e^{2}}{\varepsilon_{0}} n_{p_{e}}^{i} p_{e} \sum_{q_{e}=-\infty}^{\infty} q_{e} \sum_{j=1}^{\infty} n_{q_{e}}^{j}
\end{aligned}
$$

where $B(i)=C_{c}\left(K n_{i}\right) / 3 \pi \mu d_{p, i}$ is the mechanical mobility of particle of size $i$ and $\varepsilon_{0}$ is the permittivity of free space. The first two terms account for the birth of particles of size $i$ and charge $p_{e}$ due to collisions with smaller particles of size $j$ and charge $q_{e}$, the third and fourth terms account for the death of particles of size $i$ and charge $p_{e}$ by collisions with all other sizes, and the fifth term accounts for electrostatic dispersion. This model uses the 
geometrical discretization scheme of Hounslow et al. (1988) where the size range is discretized geometrically as

$$
d_{p, i+1} / d_{p, i}=\sqrt[3]{2}
$$

Selecting a geometric series allows for a large range of sizes to be covered (typical size distributions may cover two orders of magnitude or more) within a computationally manageable number of intervals without sacrificing accuracy at small sizes. This particular scheme also simplifies the number of ways particles coagulate into higher size intervals, and is developed in terms of particle volume (hence the cubed root) for consistency with both the zeroeth and third moment transformations (Hounslow et al. 1988). An implementation of this model written in Mathematica was borrowed from Palsmeier and Loyalka (2013) in which a modified Brownian coagulation kernel was used to account for charge effects:

$$
\beta_{p_{e}, q_{e}}^{i, j}=\beta^{i, j} \beta_{p_{e}, q_{e}}
$$

where the Brownian kernel is given by,

$$
\beta^{i, j}=2 \pi\left(\mathscr{D}_{i}+\mathscr{D}_{j}\right)\left(d_{p, i}+d_{p, j}\right) / F,
$$

with

$$
\begin{gathered}
F=\frac{d_{p, i}+d_{p, j}}{d_{p, i}+d_{p, j}+2 g_{i, j}}+\frac{8\left(\mathscr{D}_{i}+\mathscr{D}_{j}\right)}{\bar{V}_{i, j}\left(d_{p, i}+d_{p, j}\right)} \\
g_{i, j}=\sqrt{g_{i}^{2}+g_{j}^{2}} \\
\bar{V}_{i, j}=\sqrt{V_{i}^{2}+V_{j}^{2}}
\end{gathered}
$$




$$
\begin{gathered}
g_{i}=\frac{1}{3 d_{p, i} \ell_{i}}\left|\left(d_{p, i}+\ell_{i}\right)^{3}-\left(d_{p, i}^{2}+\ell_{i}^{2}\right)^{3 / 2}\right|-d_{p, i} \\
\ell_{i}=\frac{8 D_{i}}{\pi V_{i}} \\
V_{i}=\sqrt{\frac{8 k_{B} T}{\pi m_{i}}}
\end{gathered}
$$

where the agglomeration shape factor (not shown, see Gelbard 1982) has been taken to be unity and $m_{i}$ is the mass of particle of size $d_{p, i}$. The correction for Coulombic interaction is given by (Fuchs 1964)

$$
\beta_{p_{e}, q_{e}}= \begin{cases}\frac{\lambda_{p_{e}, q_{e}}}{e^{\lambda_{p_{e}, q_{e}}}-1} & \text { repulsion } \\ \frac{\left|\lambda_{p_{e}, q_{e}}\right|}{1-e^{-\left|\lambda_{p_{e}, q_{e}}\right|}} & \text { attraction }\end{cases}
$$

where

$$
\lambda_{p_{e}, q_{e}}=\frac{p_{e} q_{e} e^{2}}{2 \pi \varepsilon_{0} k_{B} T\left(d_{p, p_{e}}+d_{p, q_{e}}\right)}
$$

The initial conditions for the simulation (size and charge distribution at $t=0$ ) were specified using the experimental results at port \#1. Because the measured size distributions at all charge levels did not necessarily cover the entire size range, lognormal fits were made to the size distributions at each charge level. A total of 30 sections were used where the smallest particle size was taken to be $1 \mathrm{~nm}$, resulting in a maximum particle size of $10^{3} \mathrm{~nm}(1 \mu \mathrm{m})$. 


\subsubsection{Direct Simulation Monte Carlo}

The direct simulation Monte Carlo (DSMC) method was also used for comparison with experimental measurements. Simulations were carried out using a Mathematica program also borrowed from Palsmeier and Loyalka (2013) which used the same modified Brownian coagulation kernel as given in Eqn. 46. The size interval was divided logarithmically at a resolution of 64 divisions/decade to match the SMPS. As with the sectional method, lognormal fits to the experimental results at port \#1 were used to describe the initial distribution function. Computations were carried out using a sample size of $10^{5}$ particles generated from the specified initial distribution function and a total of ten simulations were performed to determine statistical uncertainty. Palsmeier and Loyalka (2013) provide the general order of operations for their DSMC program as:

1. A list of particles is generated by sampling from the desired initial distribution using a rejection-acceptance technique. Each list component describes an individual particle, characterized by the particle's mass and charge.

2. A time step is selected and the number of particles to be eligible for collision during that time is calculated according to the no time counter (NTC) method...

3. Two particles for potential coagulation are selected... Conservation of mass and charge means the listed properties of the particles are simply added together using Mathematica's list operation capabilities, creating a new particle, with the two original particles being deleted from the list.

4. Step 3 is repeated until a number of particles consistent with step 2 have collided. The list of particles obtained at the end of the time step replaces the list from the previous time step.

5. The entire list of particles is sampled for electrostatic dispersion corresponding with the time step described in step 2 from the data in step 4. The particle charge relative to the net charge determines whether the particle leaves the system or if a particle is gained by the system... The list that is obtained at the end of the time step can be saved for analysis.

6. Steps 2 through 5 are repeated until the desired total time for the simulation has elapsed. 
7. The sequence of data lists that are generated can then be processed to show the time-dependent evolution of the aerosol system.

8. Simulations are repeated to allow the results to be averaged and to obtain estimations of statistical errors.

No modifications to the main program were made, only minor modifications to define the initial distribution function, particle size resolution and physical properties of the system. 


\section{Chapter 4}

\section{RESULTS}

\subsection{Measured Size and Charge Distributions}

\subsubsection{Spark Generated Silver Nanoparticles}

The results for spark generated silver nanoparticles, given below in Figures 7-11, show that the aerosol had bipolar symmetry at port $\# 1$ and port $\# 2$, with the \pm 1 and \pm 2 charge levels indicating near equal magnitudes over the measured interval. Charge asymmetry became stronger at larger times as the particle concentration decreased, finally indicating a larger fraction of negatively charged particles at port \#5. The concentration of neutrally charged particles remained high over the entire size range throughout the coagulation process even at Port \#5 where the measured concentration of charged particles was quite low, indicating that the coagulation process did not lead to the typical charge equilibrium of Boltzmann or Fuchs. Results for charge levels \pm 3 and higher had concentrations too low to be accurately measured with the TDMA. 


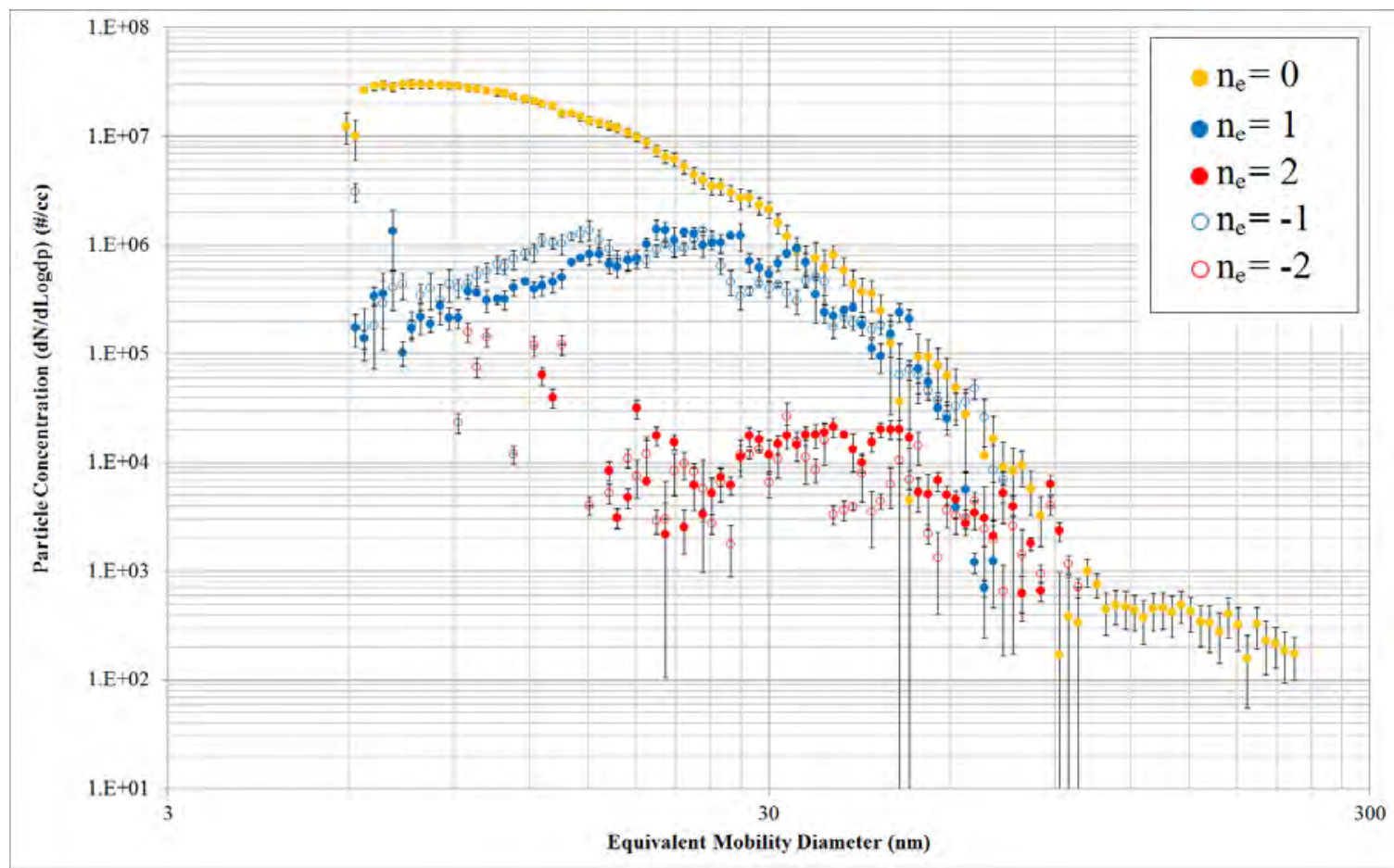

Figure 7. Measured size and charge distribution for spark generated silver nanoparticles at coagulation chamber sampling port $\# 1(\mathrm{t}=0 \mathrm{sec})$.

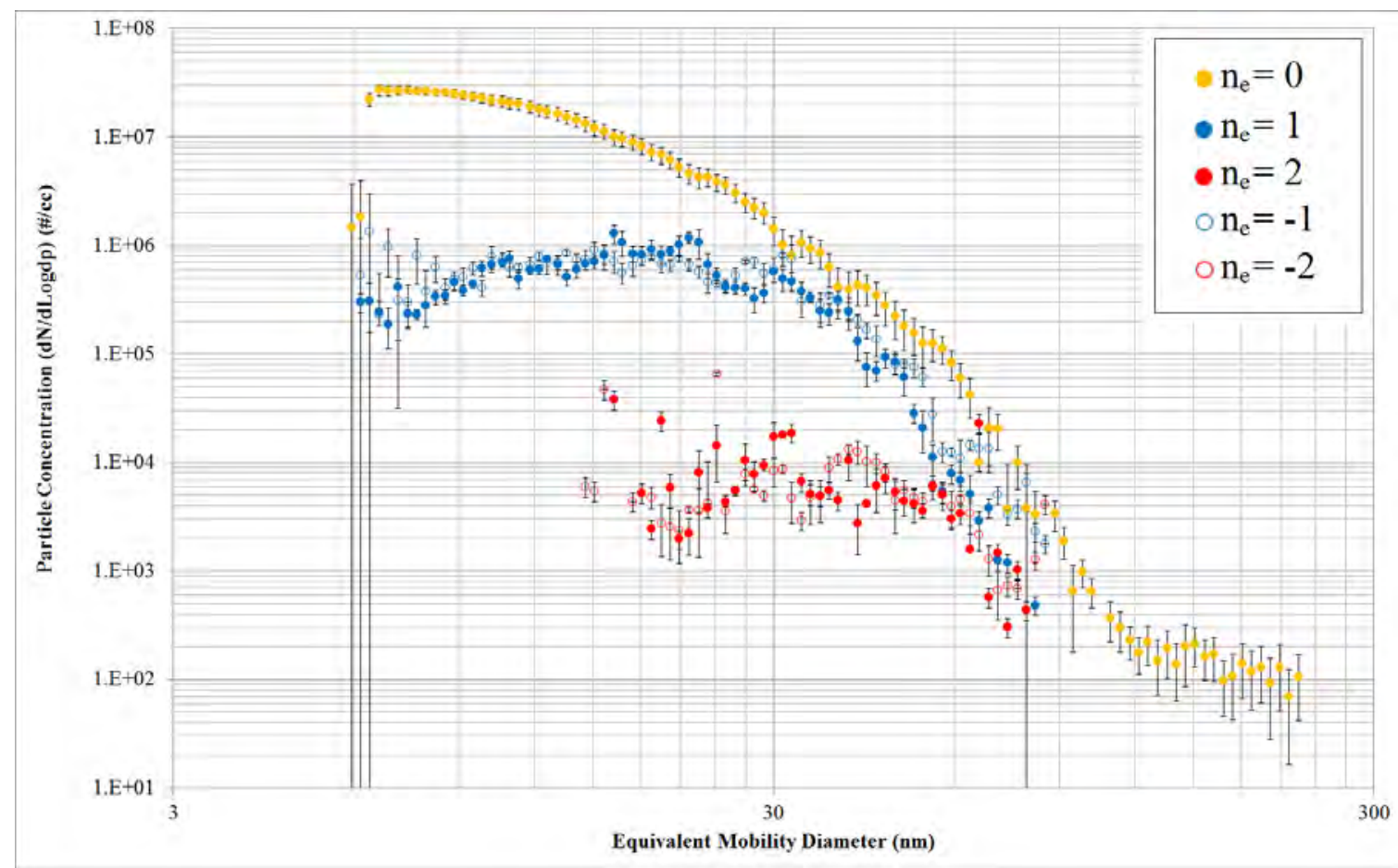

Figure 8. Measured size and charge distribution for spark generated silver nanoparticles at coagulation chamber sampling port \#2 $(\mathrm{t}=26.2 \mathrm{sec})$. 


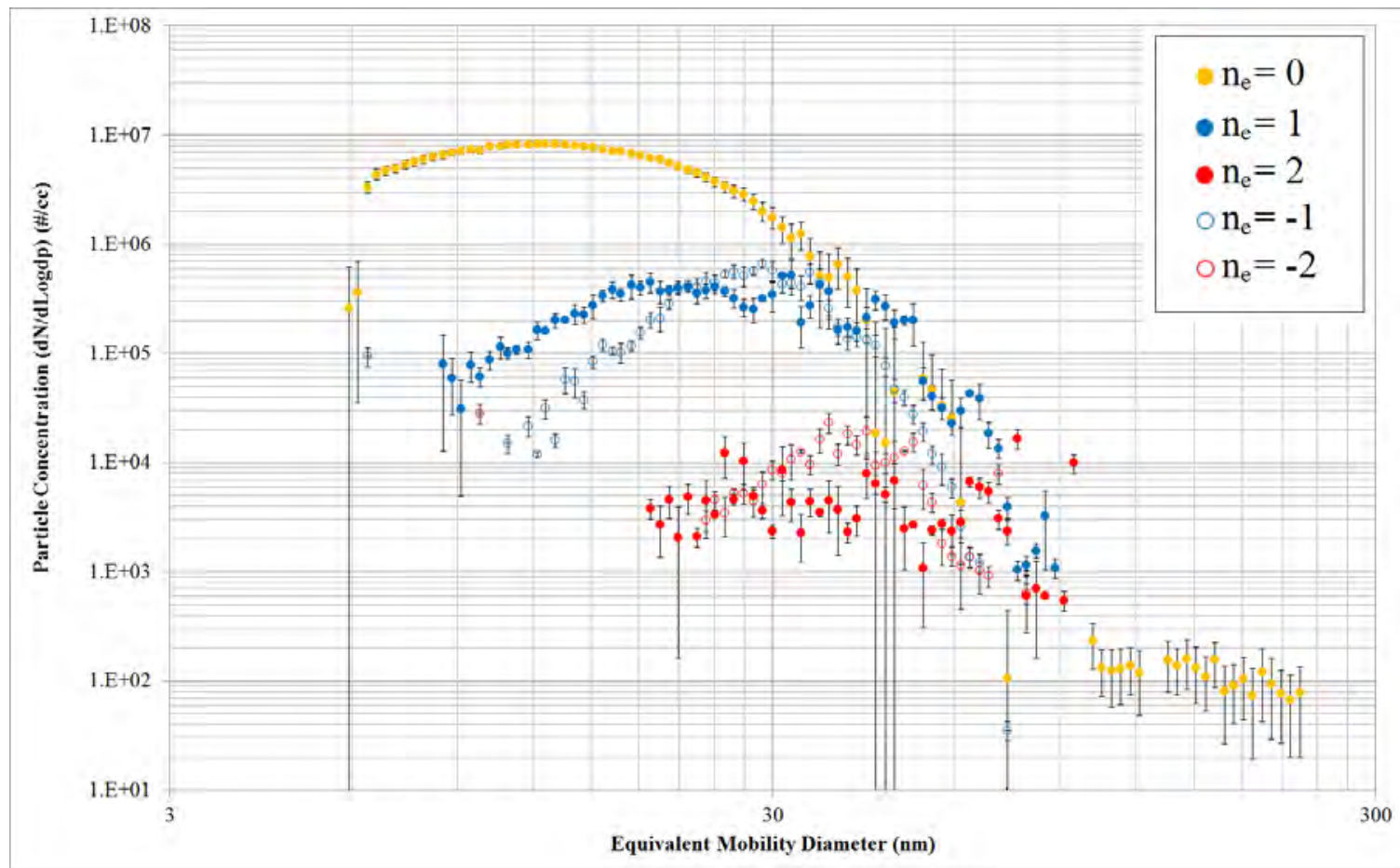

Figure 9. Measured size and charge distribution for spark generated silver nanoparticles at coagulation chamber sampling port \#3 $(\mathrm{t}=78.6 \mathrm{sec})$.

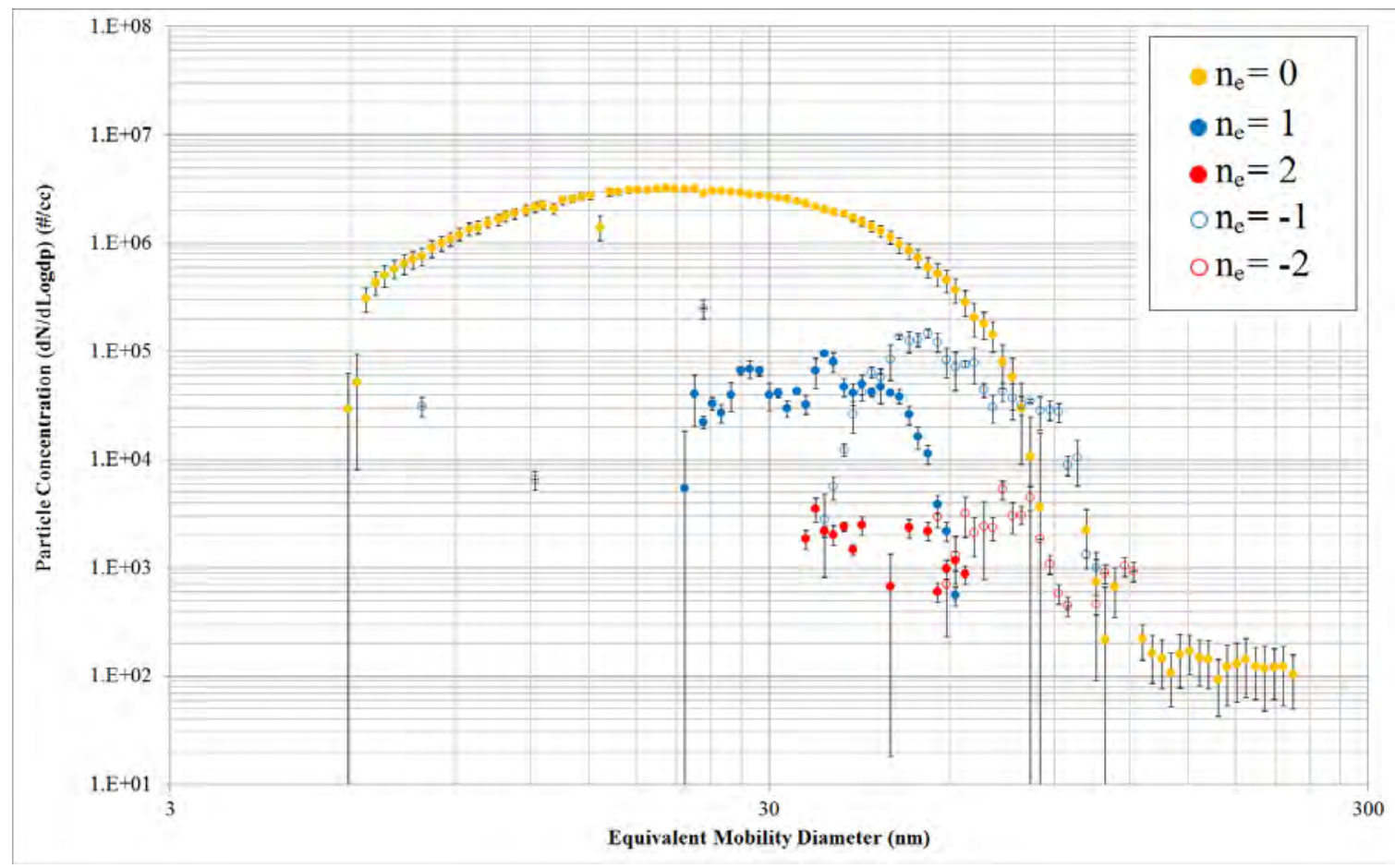

Figure 10. Measured size and charge distribution for spark generated silver nanoparticles at coagulation chamber sampling port $\# 4(\mathrm{t}=183.3 \mathrm{sec})$. 


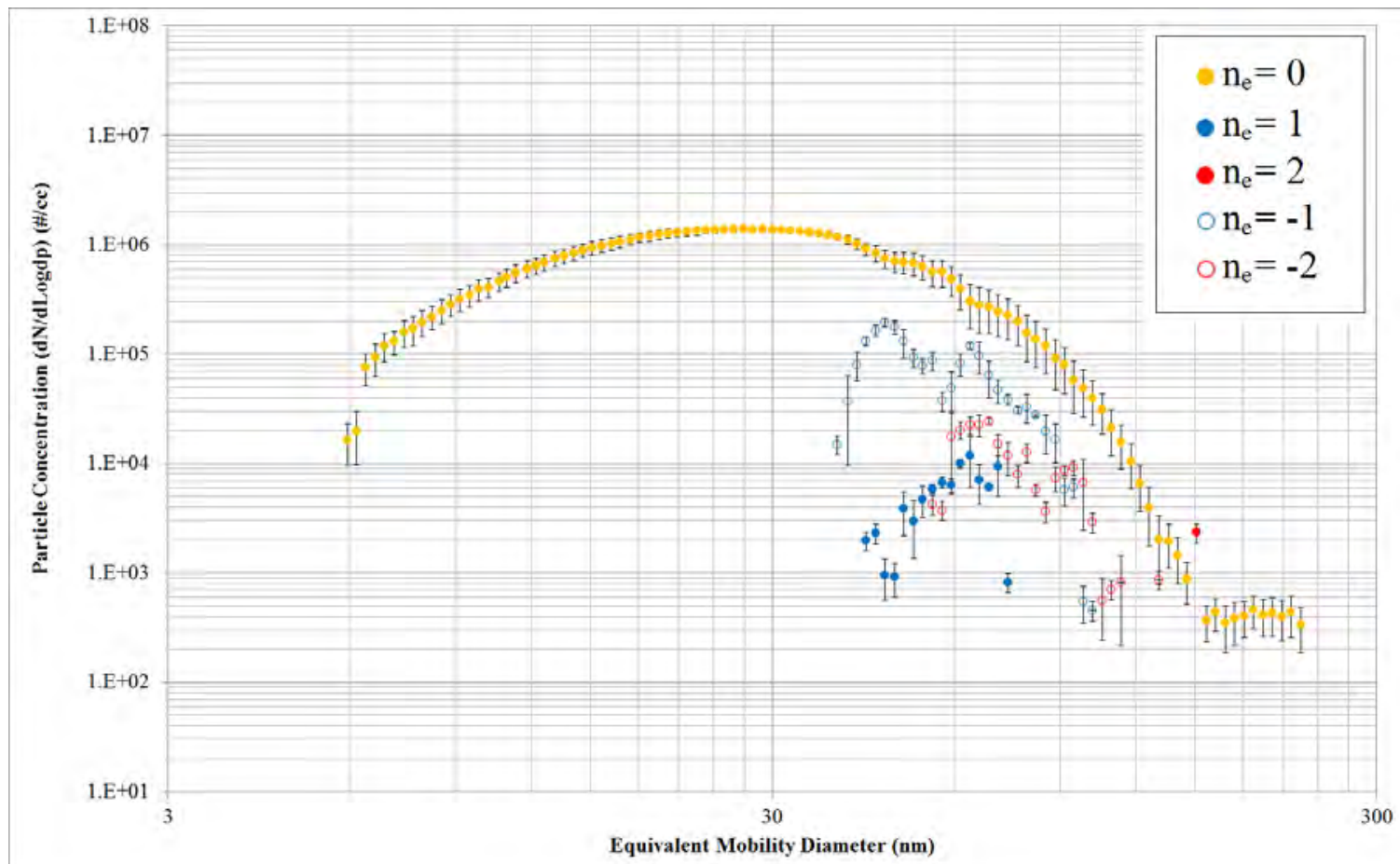

Figure 11. Measured size and charge distribution for spark generated silver nanoparticles at coagulation chamber sampling port \#5 ( $\mathrm{t}=392.8 \mathrm{sec})$. 


\subsubsection{Spark Generated Carbon Nanoparticles}

The results for spark generated carbon nanoparticles, given below in Figures 12-16, indicate strong charge asymmetry with higher concentrations of positively charged particles. Charge levels were resolved up to \pm 3 units and show a decrease in charge asymmetry over time with \pm 1 charge levels approaching concentrations of similar magnitude by Port \#5. The concentration of neutrally charged particles also remained high across the entire size range at all sampling ports. 


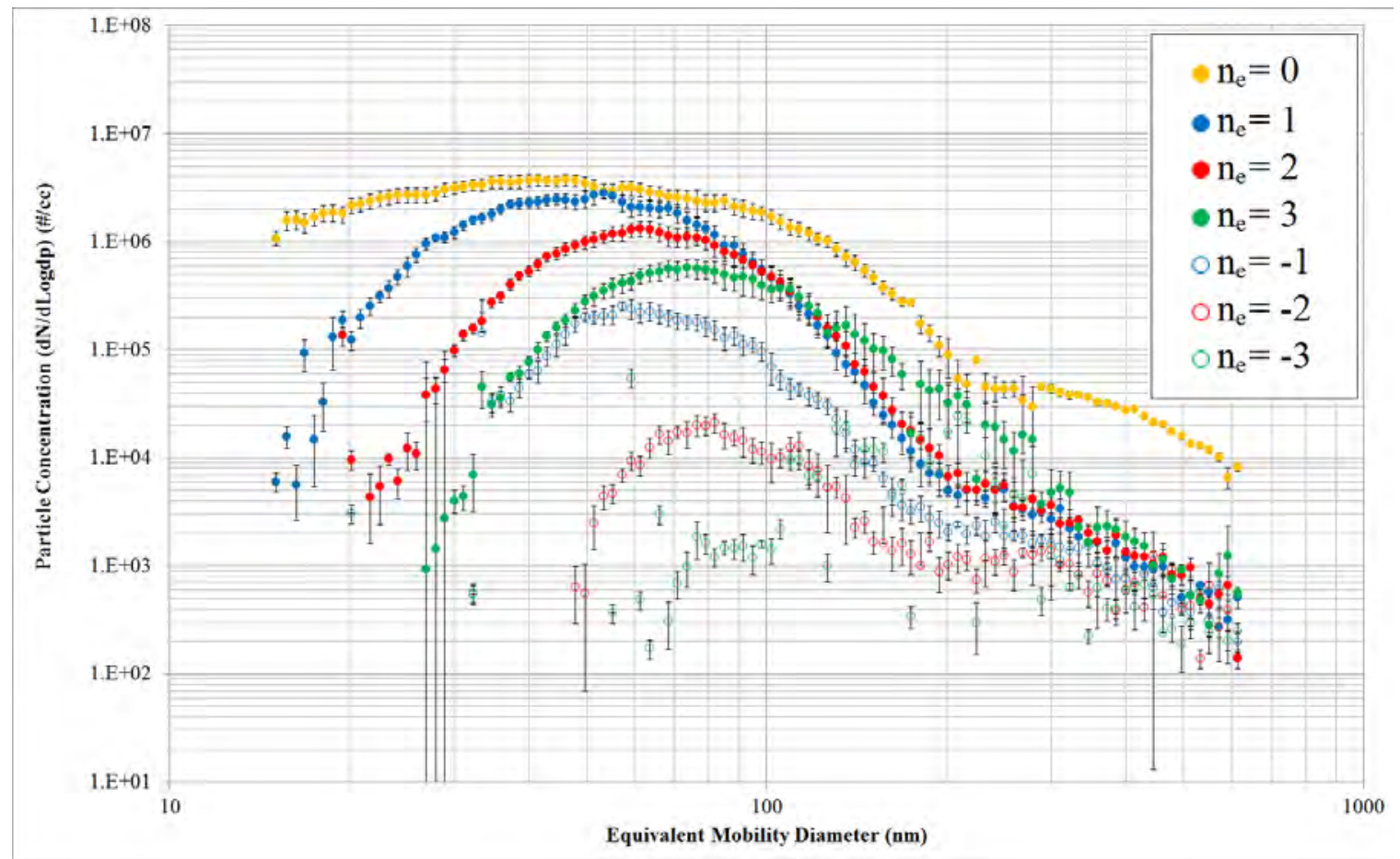

Figure 12. Measured size and charge distribution for spark generated carbon nanoparticles at coagulation chamber sampling port \#1 ( $\mathrm{t}=0 \mathrm{sec})$.

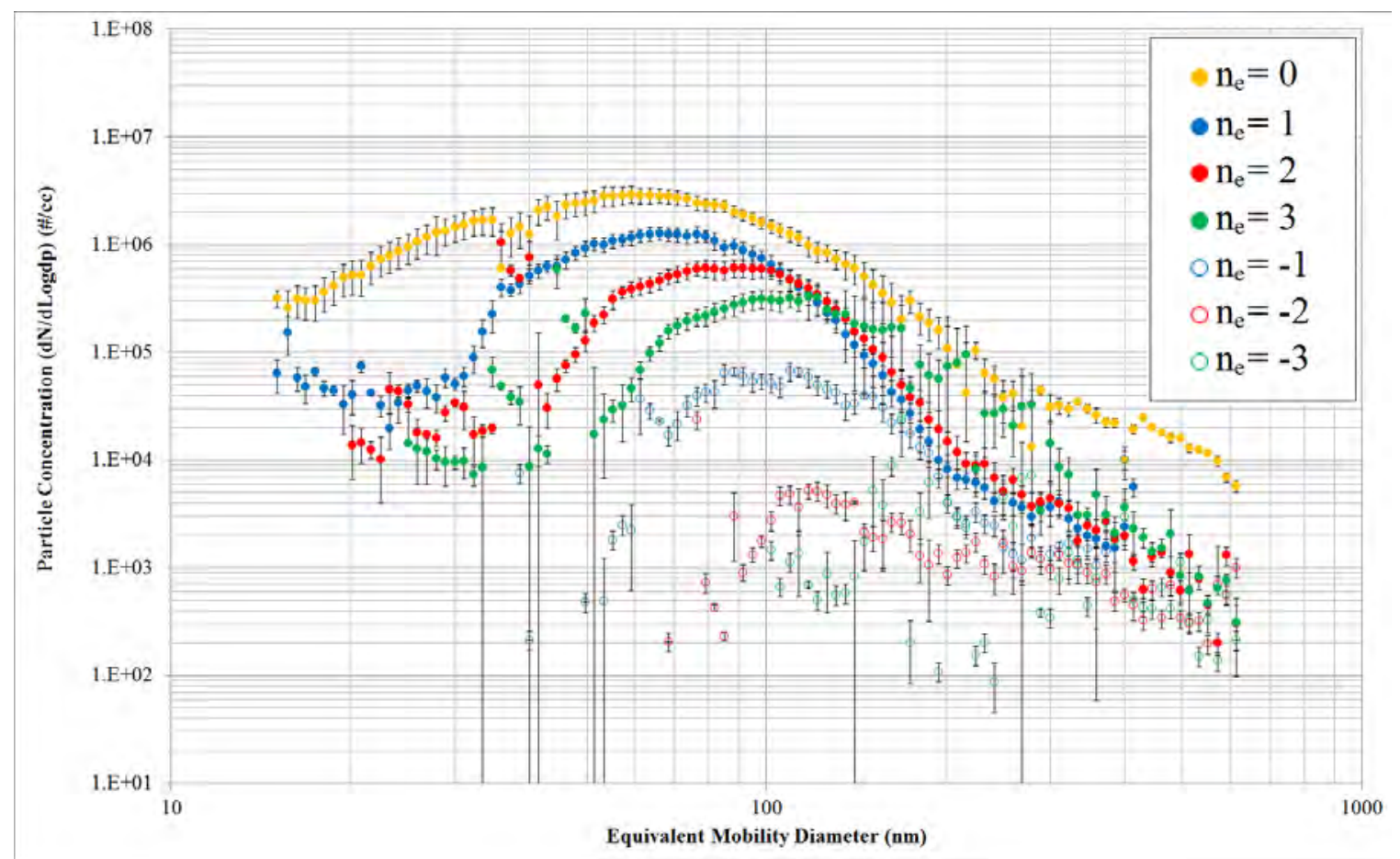

Figure 13. Measured size and charge distribution for spark generated carbon nanoparticles at coagulation chamber sampling port \#2 ( $\mathrm{t}=26.2 \mathrm{sec})$. 


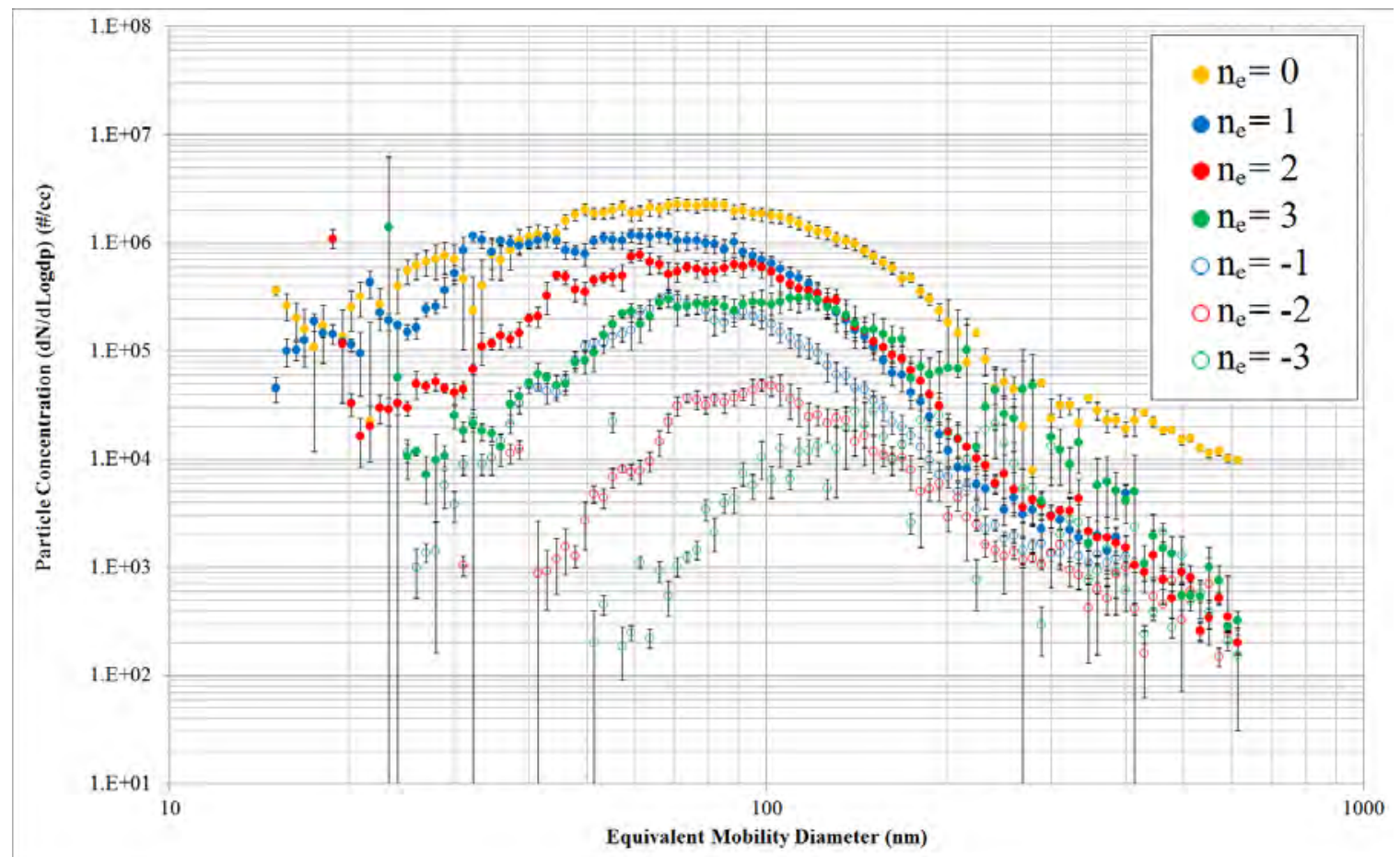

Figure 14. Measured size and charge distribution for spark generated carbon nanoparticles at coagulation chamber sampling port \#3 $(\mathrm{t}=78.6 \mathrm{sec})$.

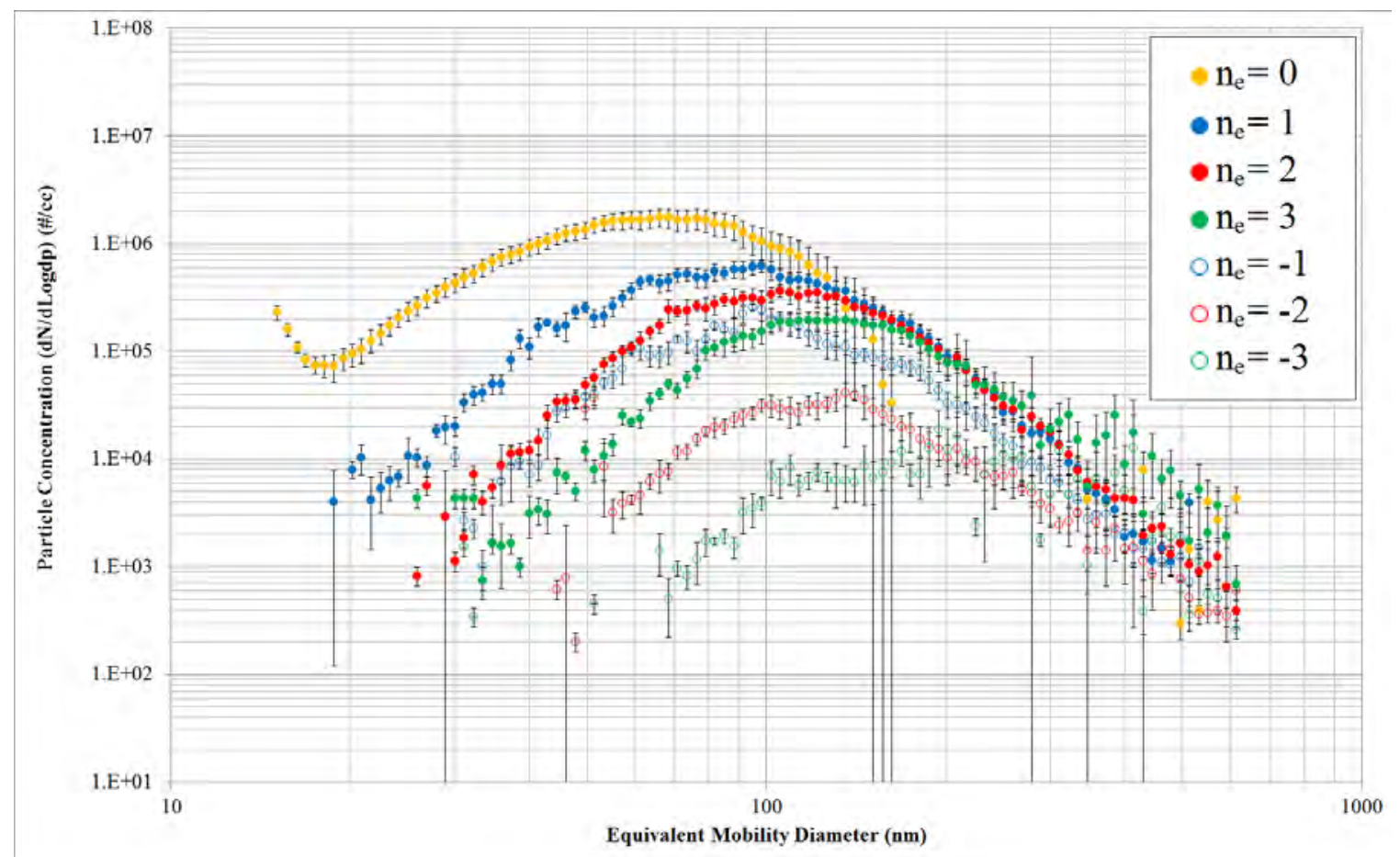

Figure 15. Measured size and charge distribution for spark generated carbon nanoparticles at coagulation chamber sampling port $\# 4(\mathrm{t}=183.3 \mathrm{sec})$. 


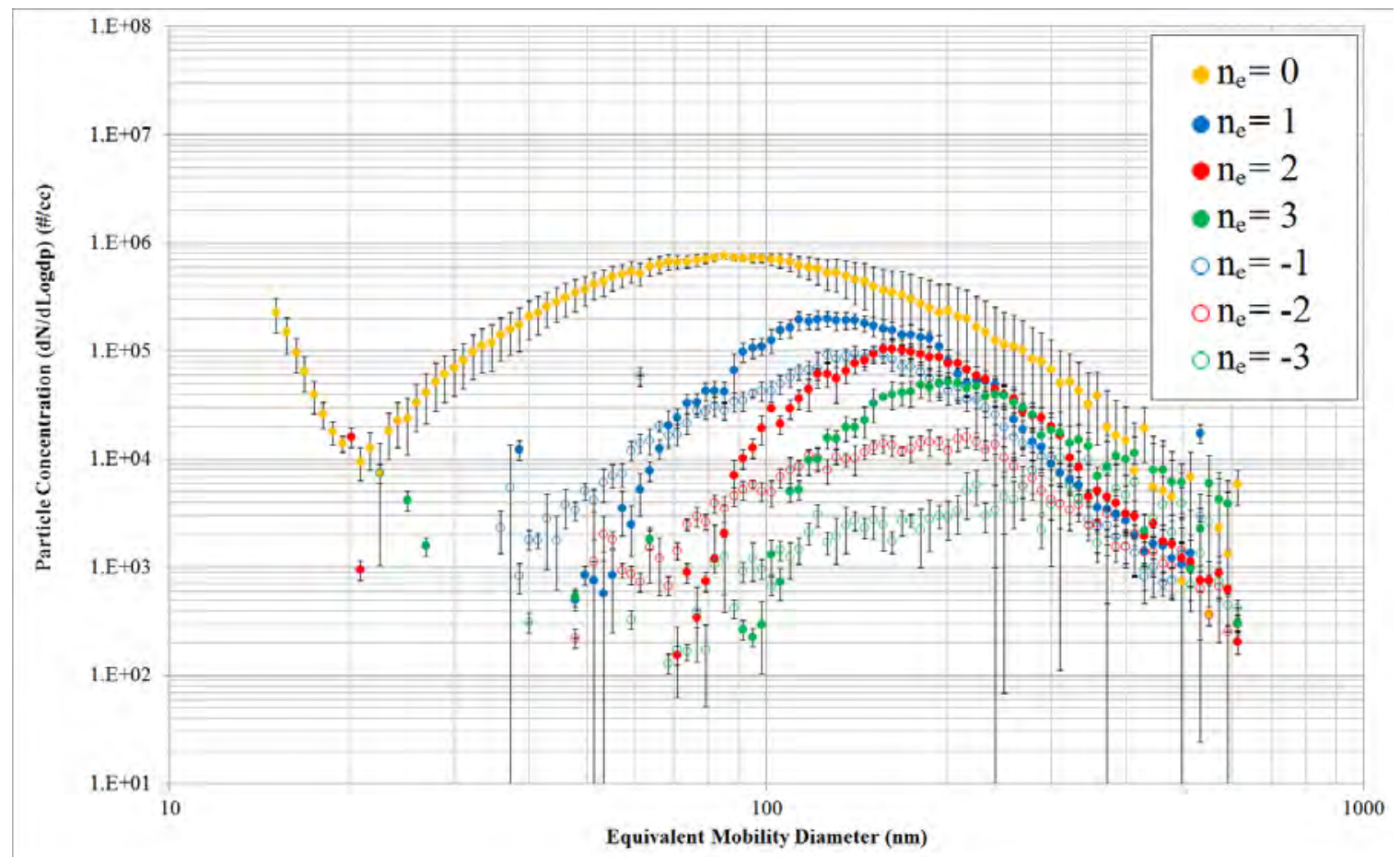

Figure 16. Measured size and charge distribution for spark generated carbon nanoparticles at coagulation chamber sampling port \#5 ( $\mathrm{t}=392.8 \mathrm{sec})$. 


\subsection{Comparison of Sectional Method with Measurements}

\subsubsection{Silver}

The predictions of the coagulation model using the sectional method are shown in Figures 17-21. The distributions at Port \#1 fit well with the lognormal approximations for the initial conditions of the sectional model. Results at sampling Port \#2 indicate that the sectional method under predicted values measured at sizes less than $15 \mathrm{~nm}$, while at Port \#3 the sectional results were in agreement again with the neutral distribution but over predicted the distribution of the -1 charge level. At Ports $\# 4 \& \# 5$ the shape of the neutral distribution became more broad causing an under prediction of the sectional method at sizes larger than $30 \mathrm{~nm}$. Gross deviations in the distribution of charged particles was also found at Ports \#4 \& \#5 with over prediction of number concentrations and much wider distribution with particle sizes smaller than measured. 


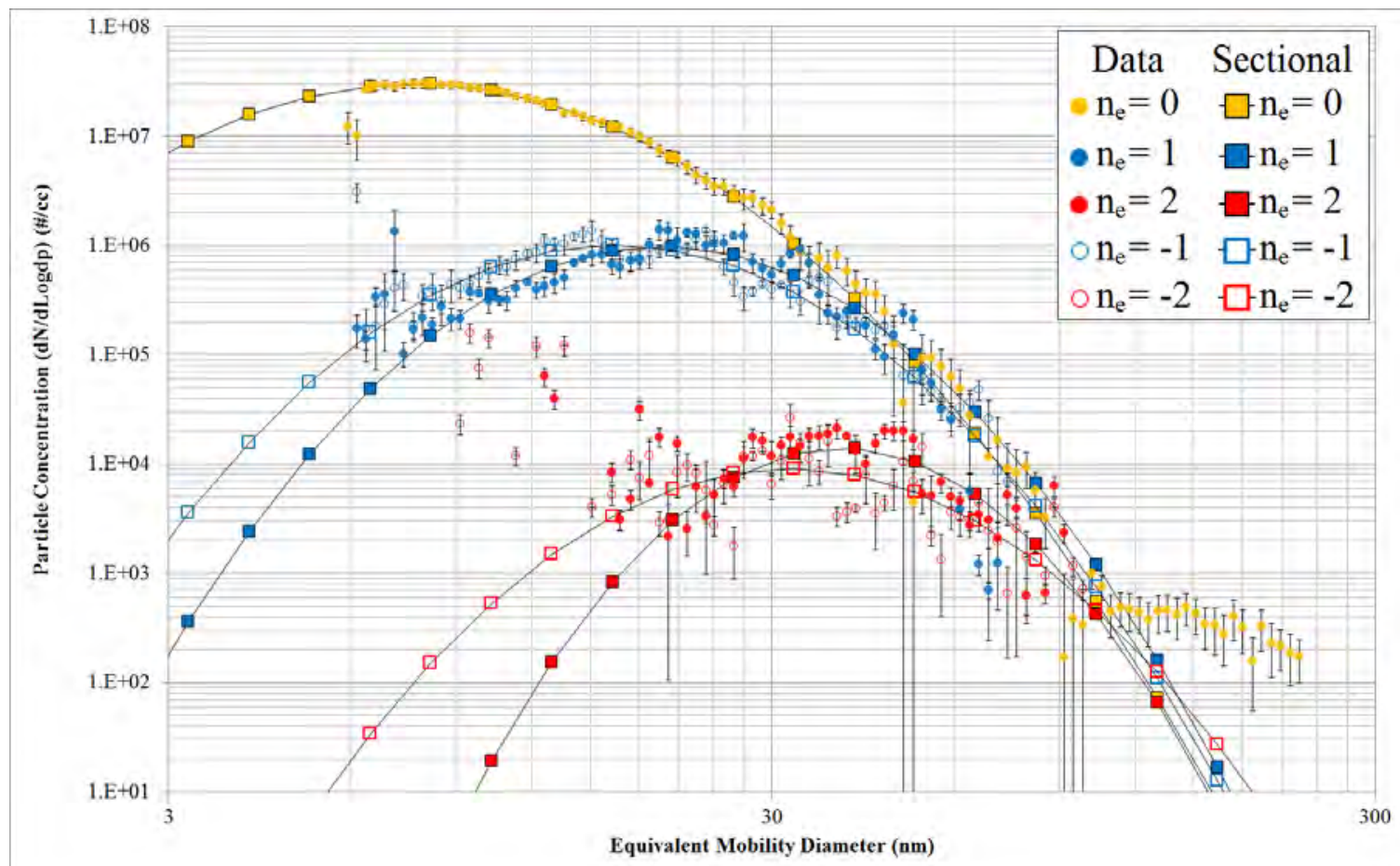

Figure 17. Comparison between the measured size and charge distribution for spark generated silver nanoparticles at coagulation chamber sampling port $\# 1(\mathrm{t}=0 \mathrm{sec})$ and the coagulation model using the sectional method.

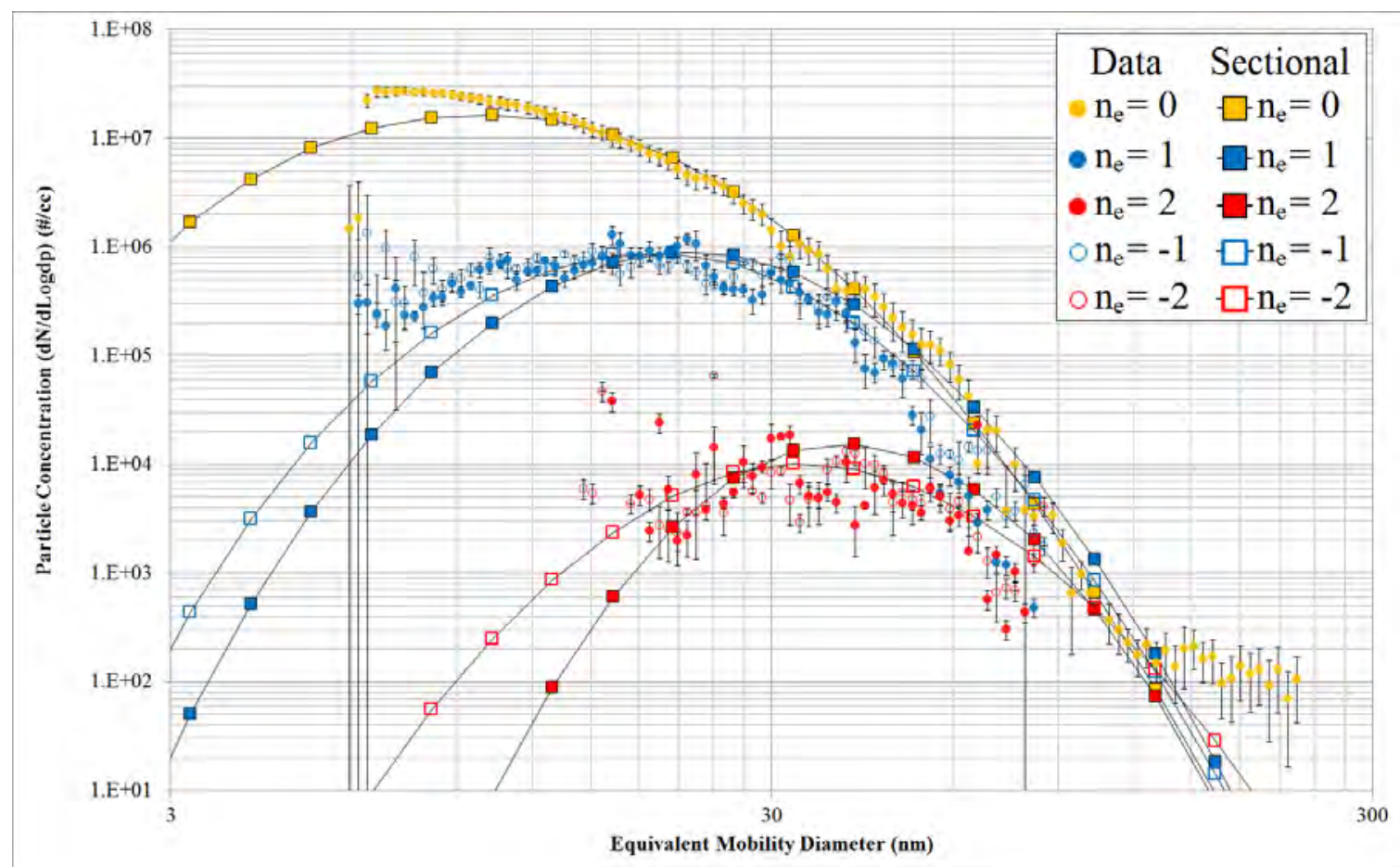

Figure 18. Comparison between the measured size and charge distribution for spark generated silver nanoparticles at coagulation chamber sampling port $\# 2(\mathrm{t}=26.2 \mathrm{sec})$ and the coagulation model using the sectional method. 


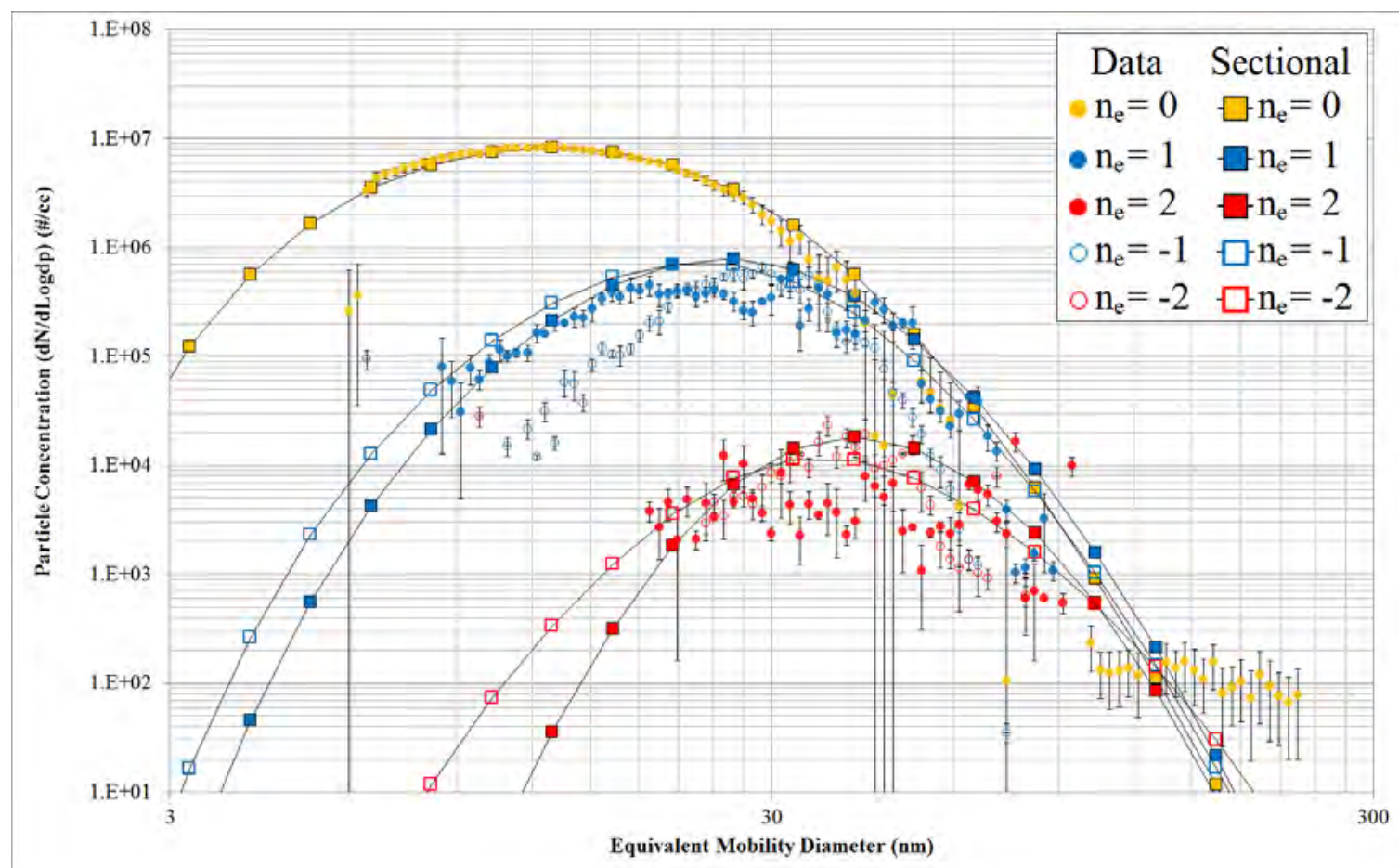

Figure 19. Comparison between the measured size and charge distribution for spark generated silver nanoparticles at coagulation chamber sampling port \#3 $(\mathrm{t}=78.6 \mathrm{sec})$ and the coagulation model using the sectional method.

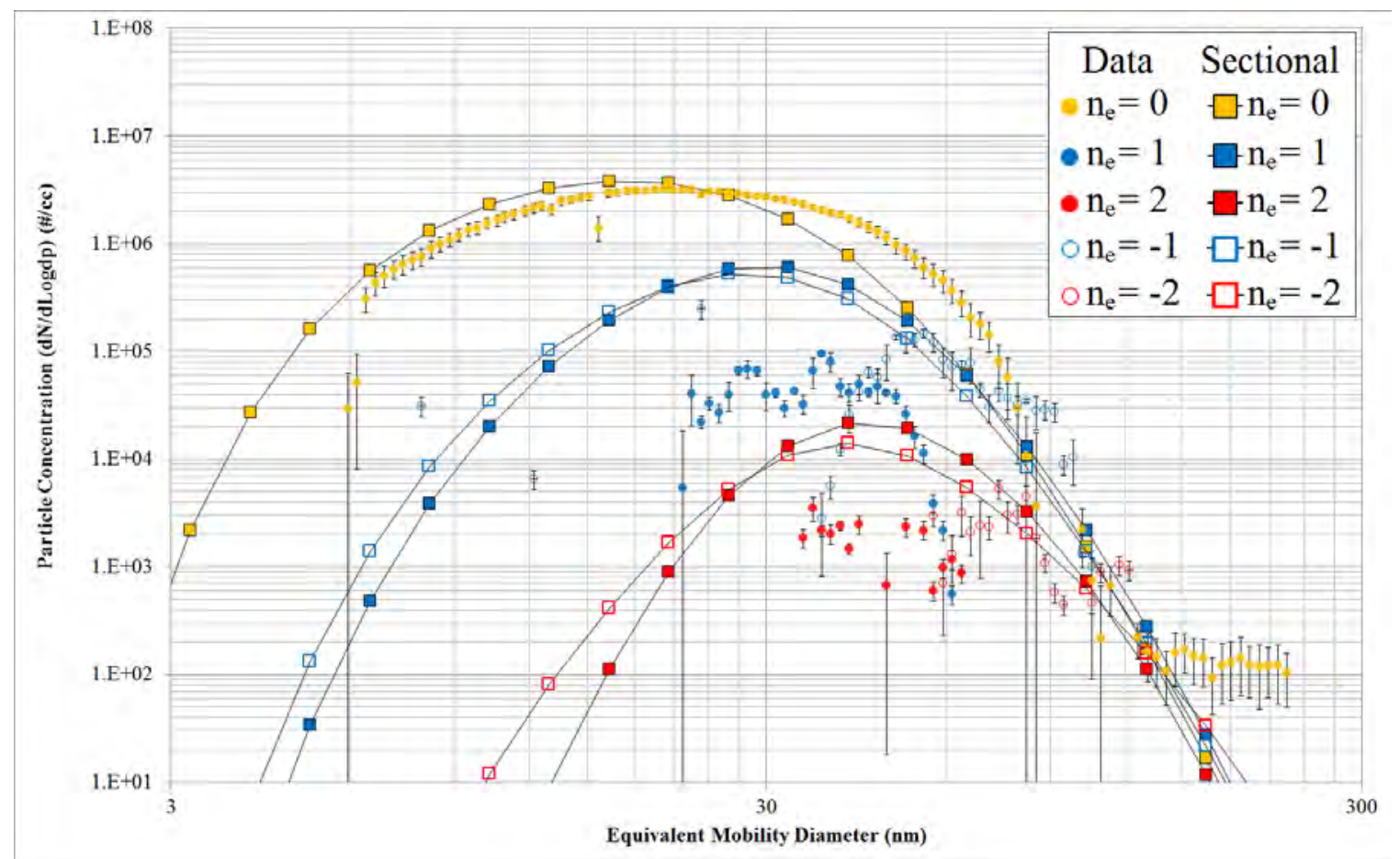

Figure 20. Comparison between the measured size and charge distribution for spark generated silver nanoparticles at coagulation chamber sampling port $\# 4(\mathrm{t}=183.3 \mathrm{sec})$ and the coagulation model using the sectional method. 


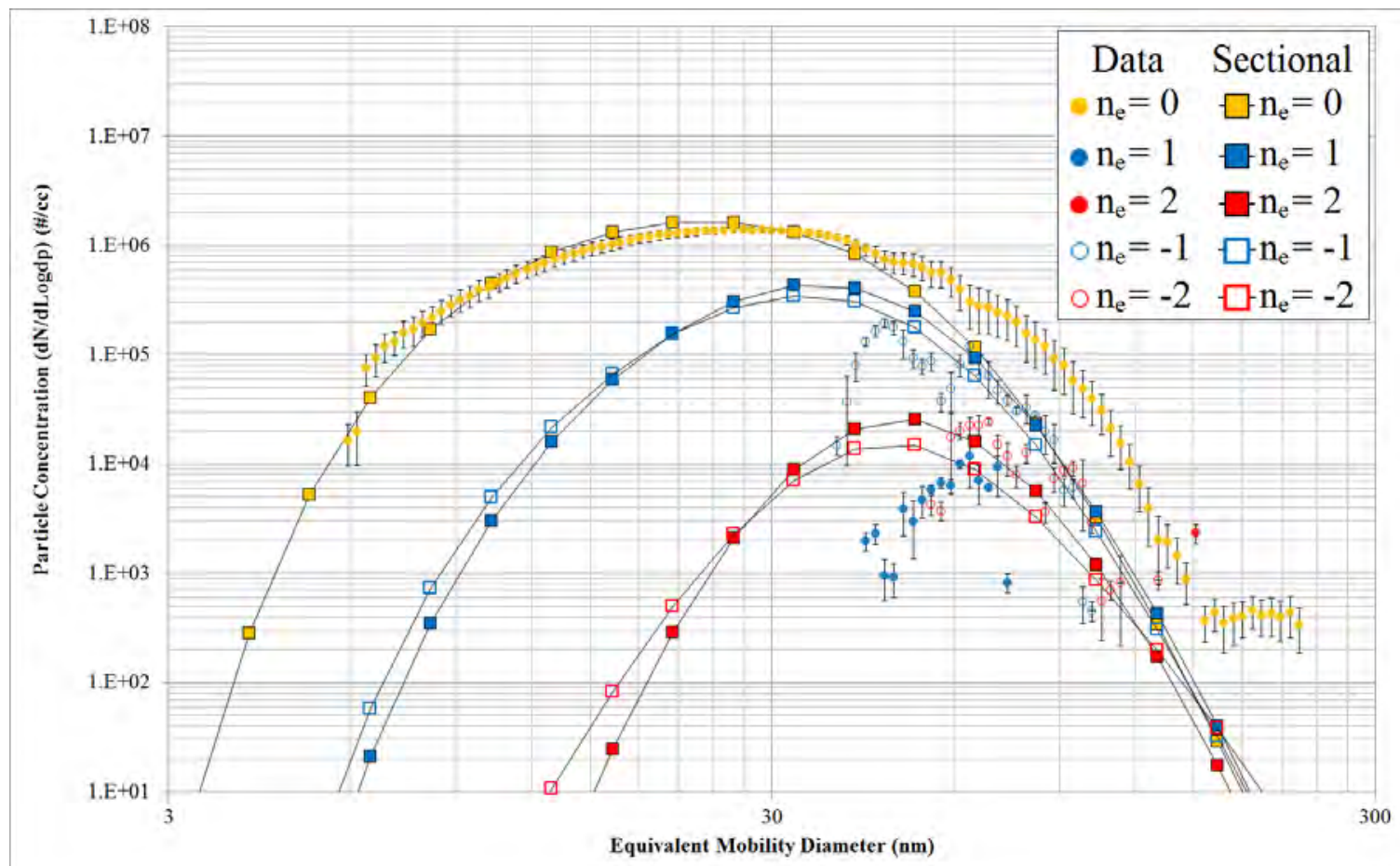

Figure 21. Comparison between the measured size and charge distribution for spark generated silver nanoparticles at coagulation chamber sampling port $\# 5(t=392.8 \mathrm{sec})$ and the coagulation model using the sectional method. 


\subsubsection{Carbon}

The predictions of the coagulation model using the sectional method are shown in Figures 22-26. Results at Port \#2 indicated an over prediction by the sectional method at all charge levels below $200 \mathrm{~nm}$ and an under prediction at sizes above $200 \mathrm{~nm}$. At Port \#3 the sectional results were in close agreement with positively charged particle distributions while negatively charged particle distributions and the neutral particle distribution were both under predicted. Sampling Ports \#4 \& \#5 show an over prediction by the model for all positively charged distributions and an under prediction by negatively and neutrally charged particle distributions. The neutral distribution predicted at Port \#5 was also shifted toward larger values than measured by the TDMA. 


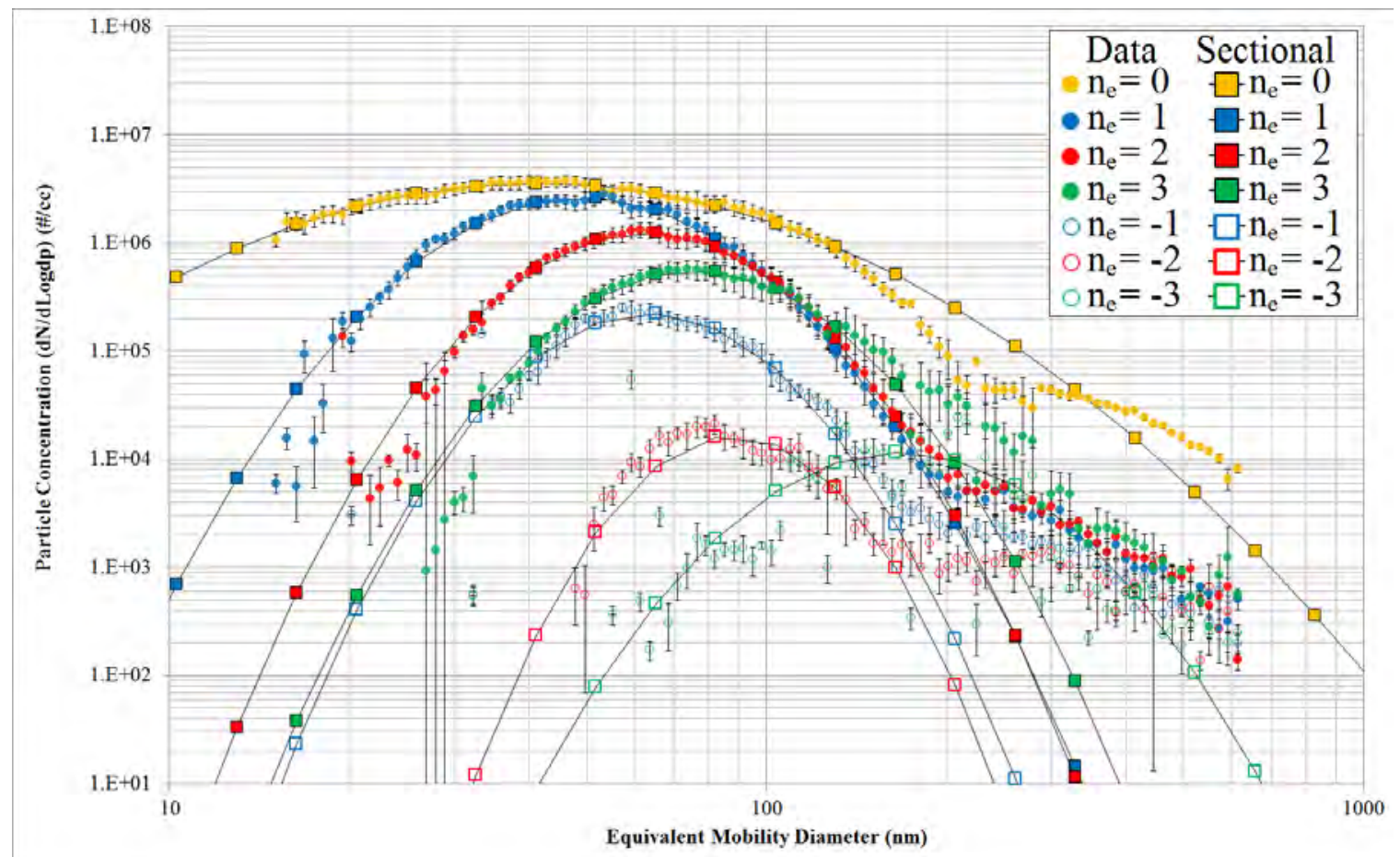

Figure 22. Comparison between the measured size and charge distribution for spark generated carbon nanoparticles at coagulation chamber sampling port \#1 ( $\mathrm{t}=0 \mathrm{sec})$ and the coagulation model using the sectional method.

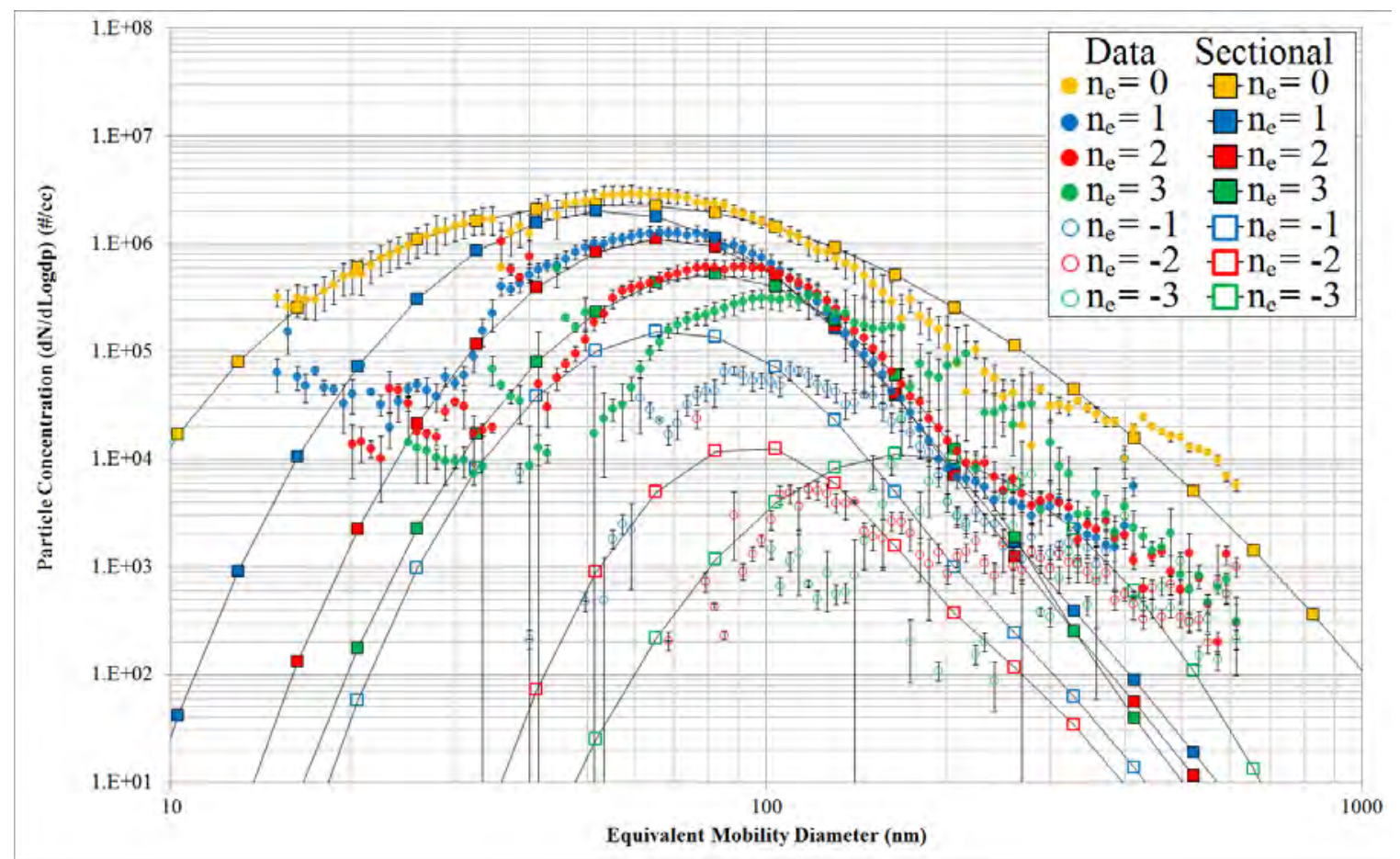

Figure 23. Comparison between the measured size and charge distribution for spark generated carbon nanoparticles at coagulation chamber sampling port \#2 $(\mathrm{t}=26.2 \mathrm{sec})$ and the coagulation model using the sectional method. 


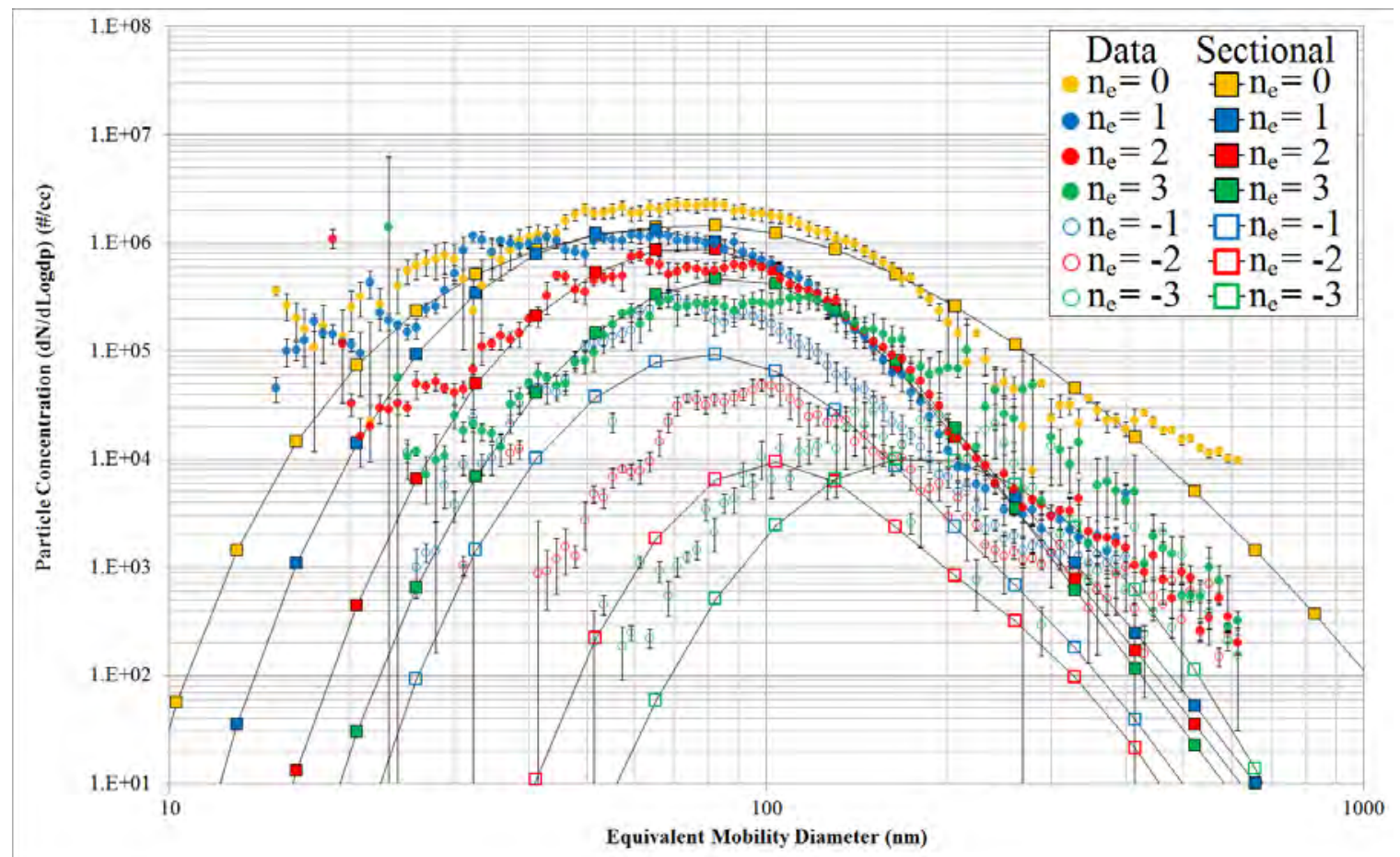

Figure 24. Comparison between the measured size and charge distribution for spark generated carbon nanoparticles at coagulation chamber sampling port \#3 $(\mathrm{t}=78.6 \mathrm{sec})$ and the coagulation model using the sectional method.

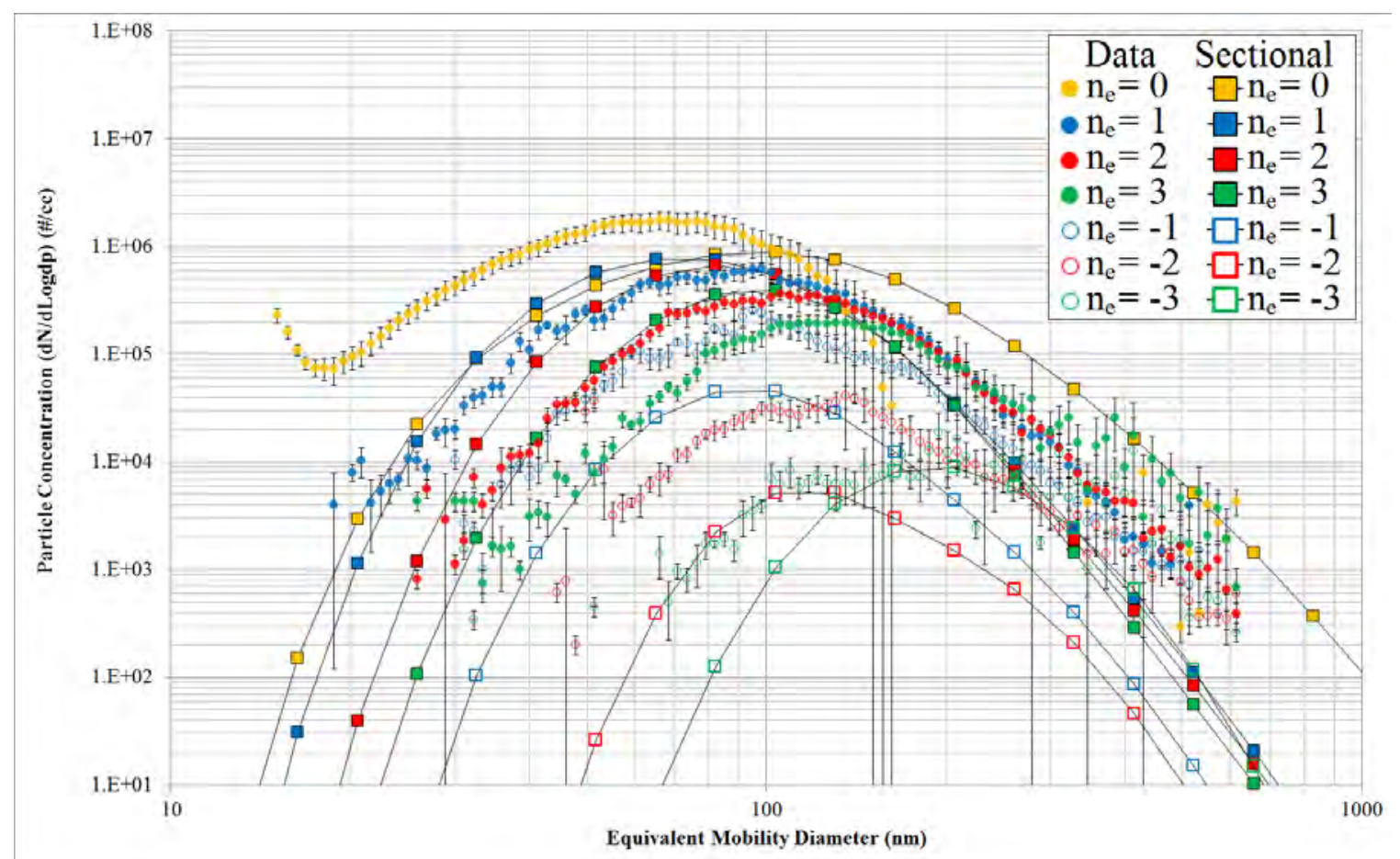

Figure 25. Comparison between the measured size and charge distribution for spark generated carbon nanoparticles at coagulation chamber sampling port \#4 ( $\mathrm{t}=183.3 \mathrm{sec})$ and the coagulation model using the sectional method. 


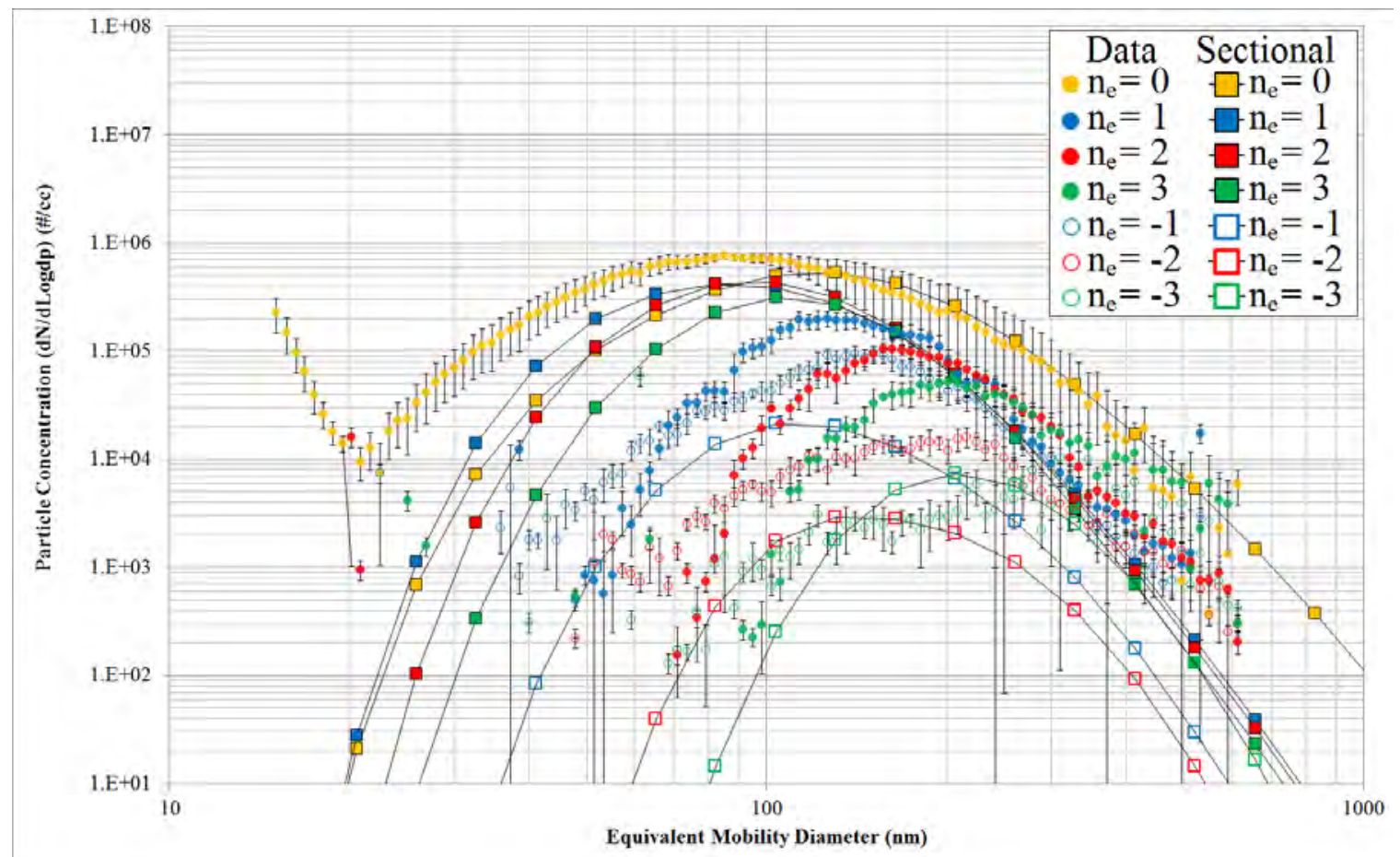

Figure 26. Comparison between the measured size and charge distribution for spark generated carbon nanoparticles at coagulation chamber sampling port \#5 ( $\mathrm{t}=392.8 \mathrm{sec})$ and the coagulation model using the sectional method. 


\subsection{Comparison of DSMC Method with Measurements}

\subsubsection{Silver}

DSMC results showed good agreement with measurements at Port \#2 after short interaction times, while results at Port \#3 over predicted measurements at all charge levels. At Port \#3 the model also predicted higher concentrations of negatively charged particles than positively charged particles at the \pm 1 charge level while measurements indicated the exact opposite. DSMC results at Port \#4 continued to be an over prediction. Differences in the overall shape between the model and measurements also became apparent at Port \#4, with measurements indicating a flatter distribution of particles than predicted. The trends found at Port \#4 continue at Port \#5 where the concentrations of charged particles measured are much lower than model predictions. 


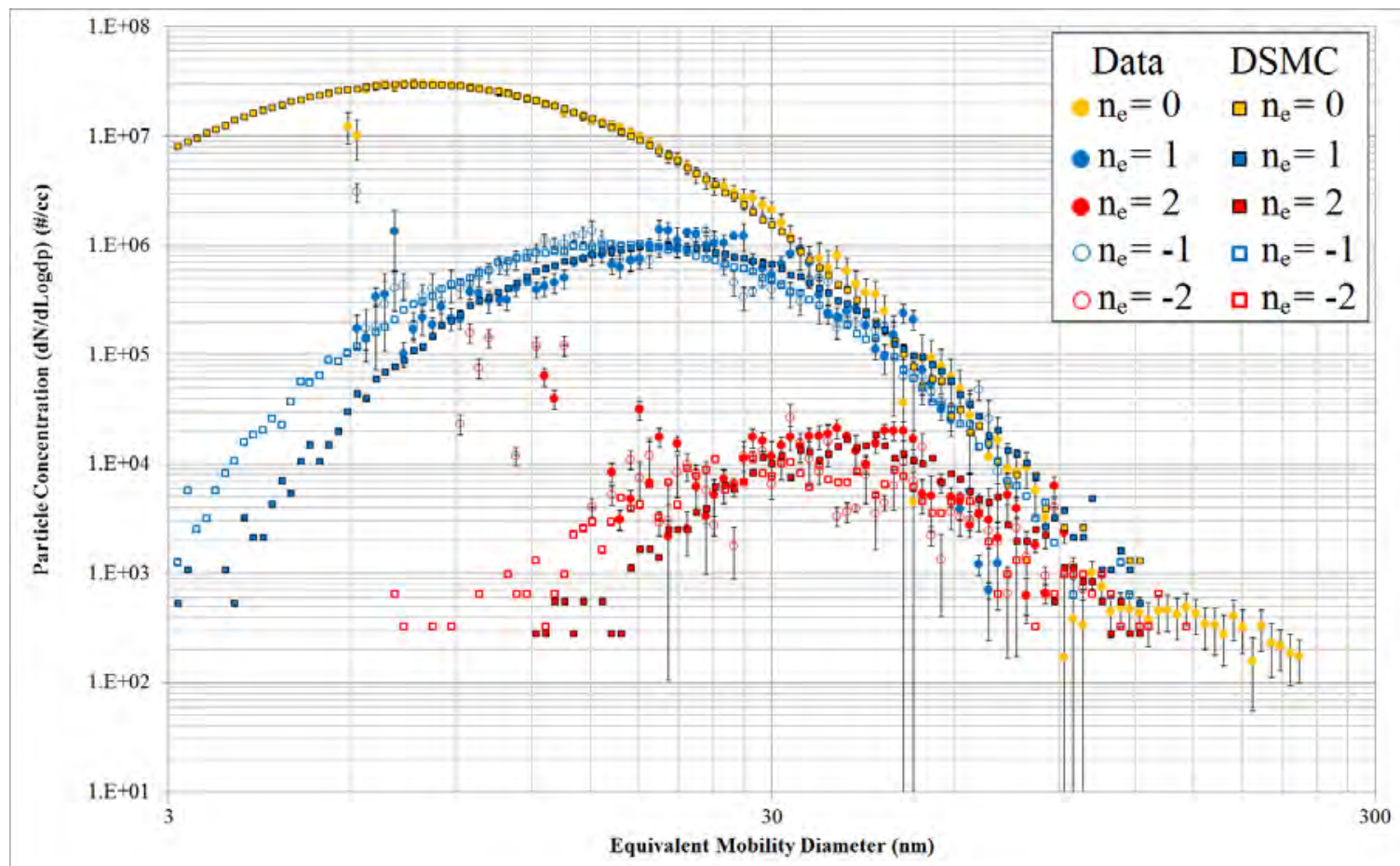

Figure 27. Comparison between the measured size and charge distribution for spark generated silver nanoparticles at coagulation chamber sampling port $\# 1(\mathrm{t}=0 \mathrm{sec})$ and the coagulation model using the DSMC method.

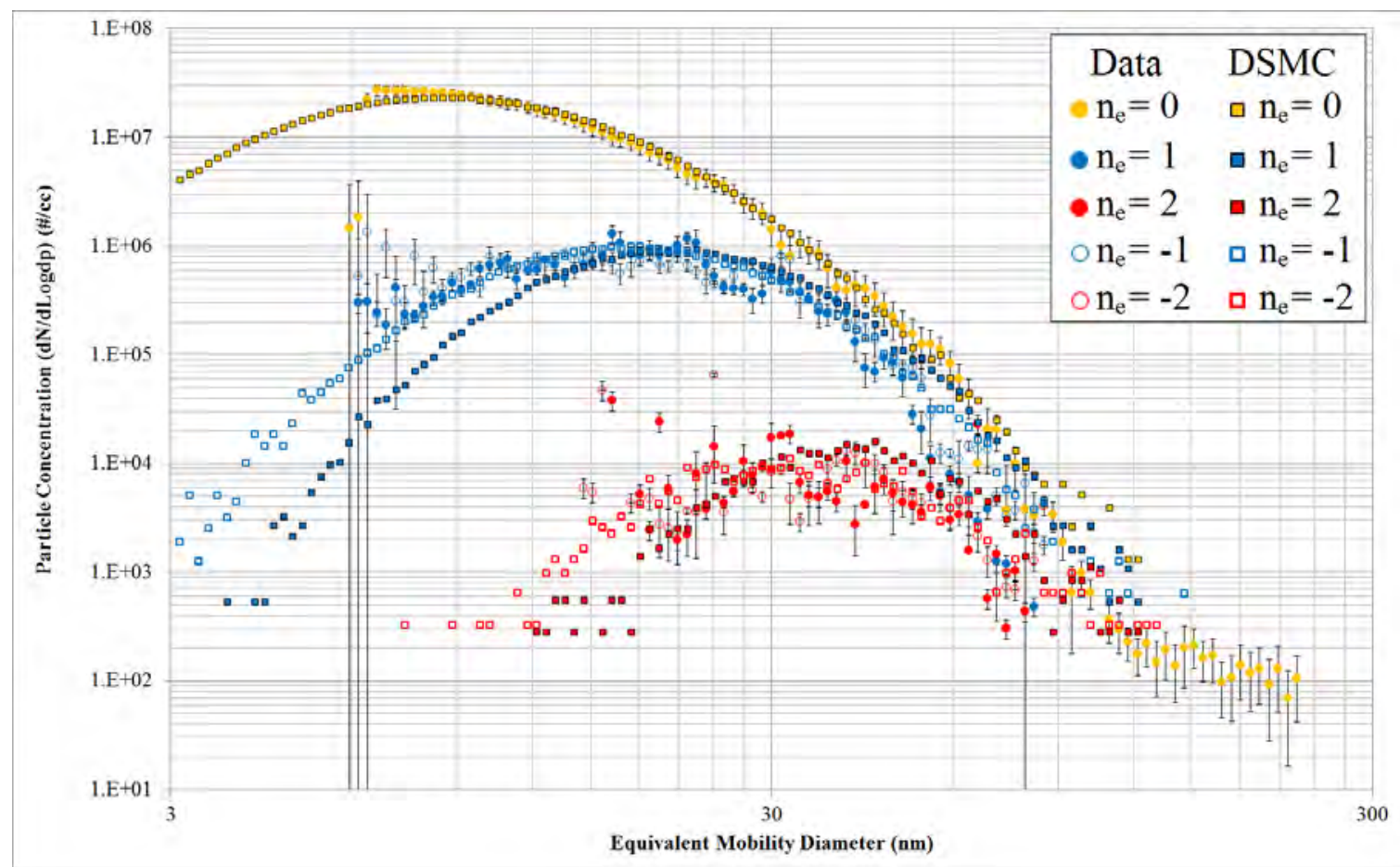

Figure 28. Comparison between the measured size and charge distribution for spark generated silver nanoparticles at coagulation chamber sampling port $\# 2(t=26.2 \mathrm{sec})$ and the coagulation model using the DSMC method. 


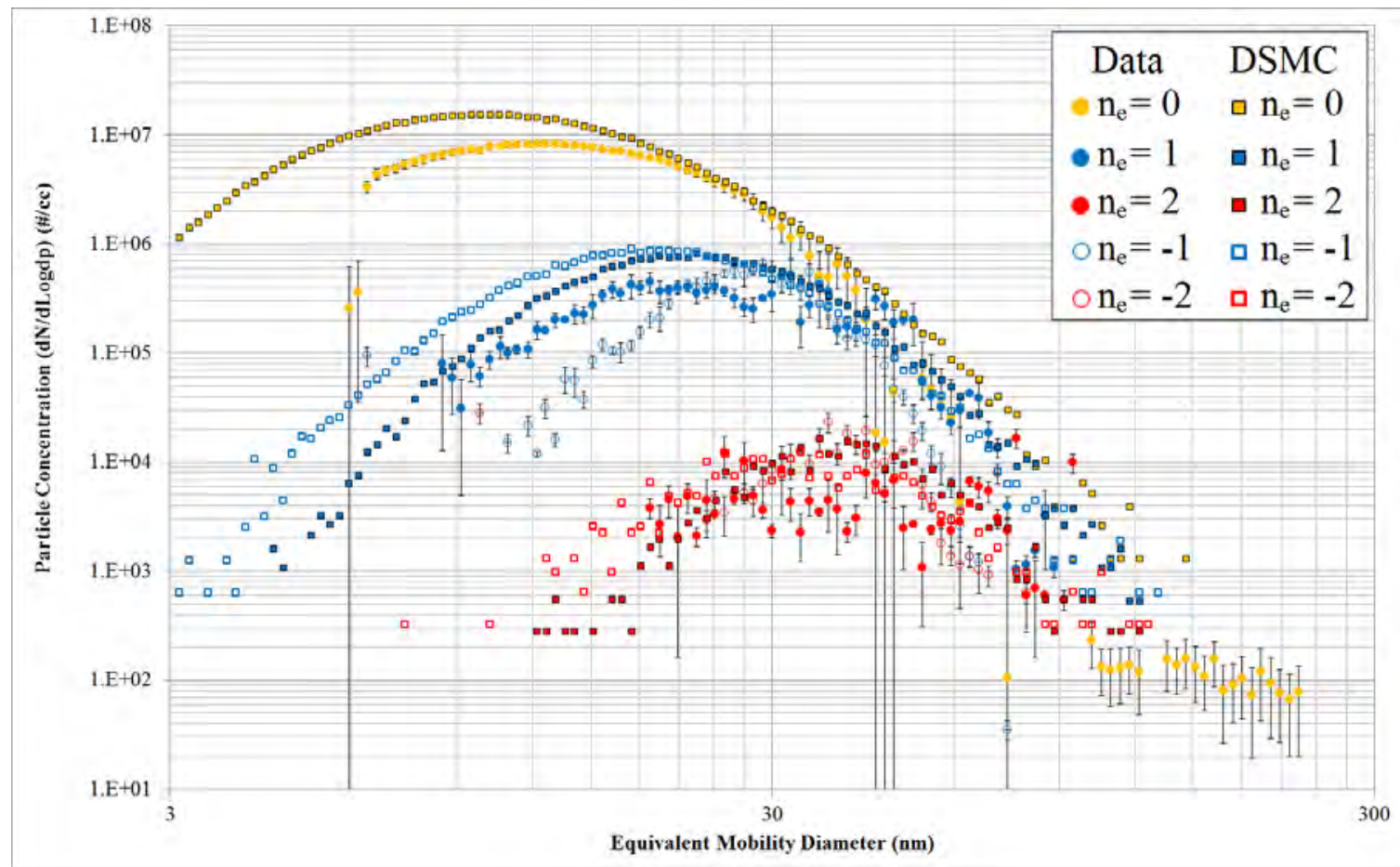

Figure 29. Comparison between the measured size and charge distribution for spark generated silver nanoparticles at coagulation chamber sampling port \#3 $(\mathrm{t}=78.6 \mathrm{sec})$ and the coagulation model using the DSMC method.

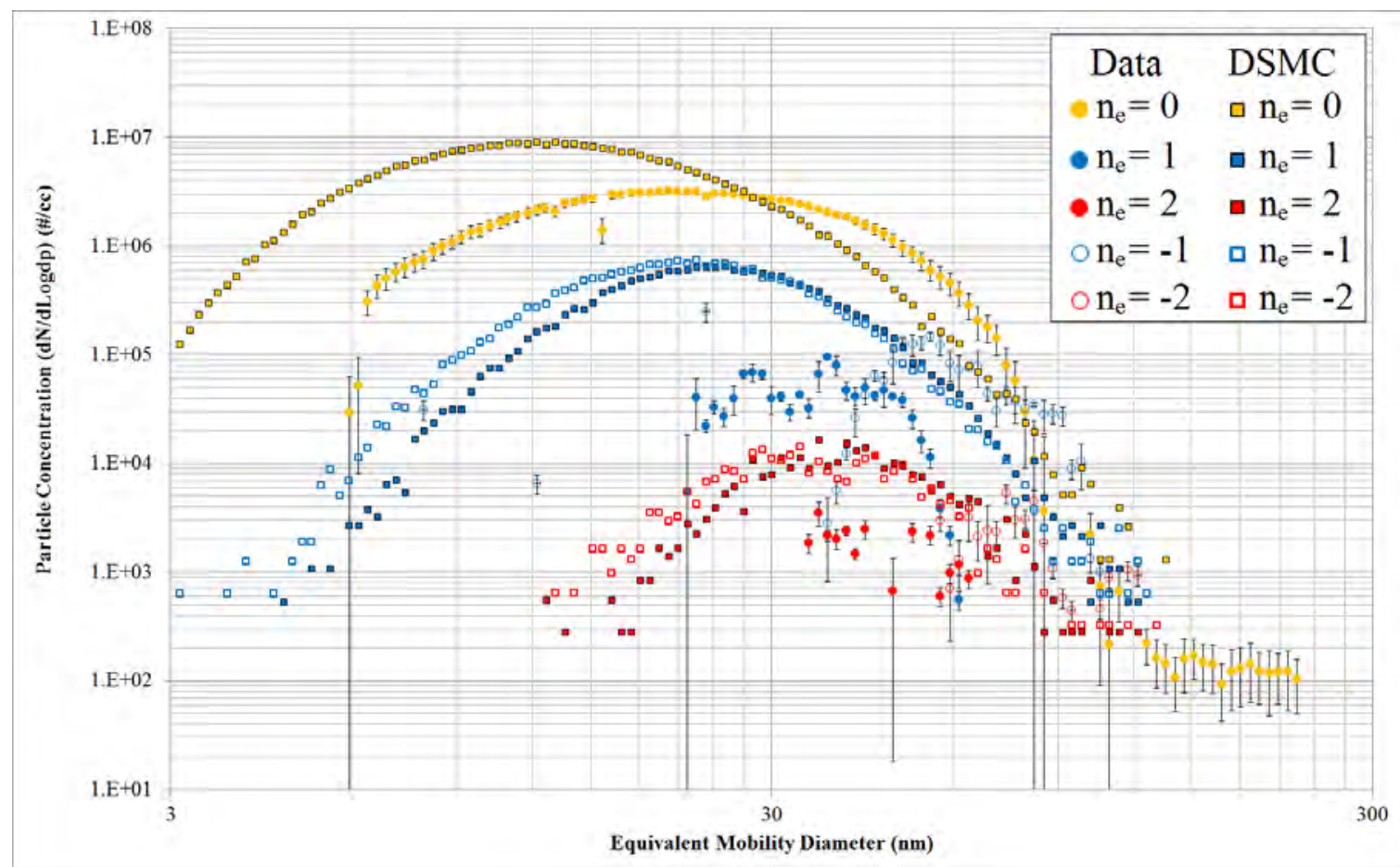

Figure 30. Comparison between the measured size and charge distribution for spark generated silver nanoparticles at coagulation chamber sampling port \#4 $(\mathrm{t}=183.3 \mathrm{sec})$ and the coagulation model using the DSMC method. 


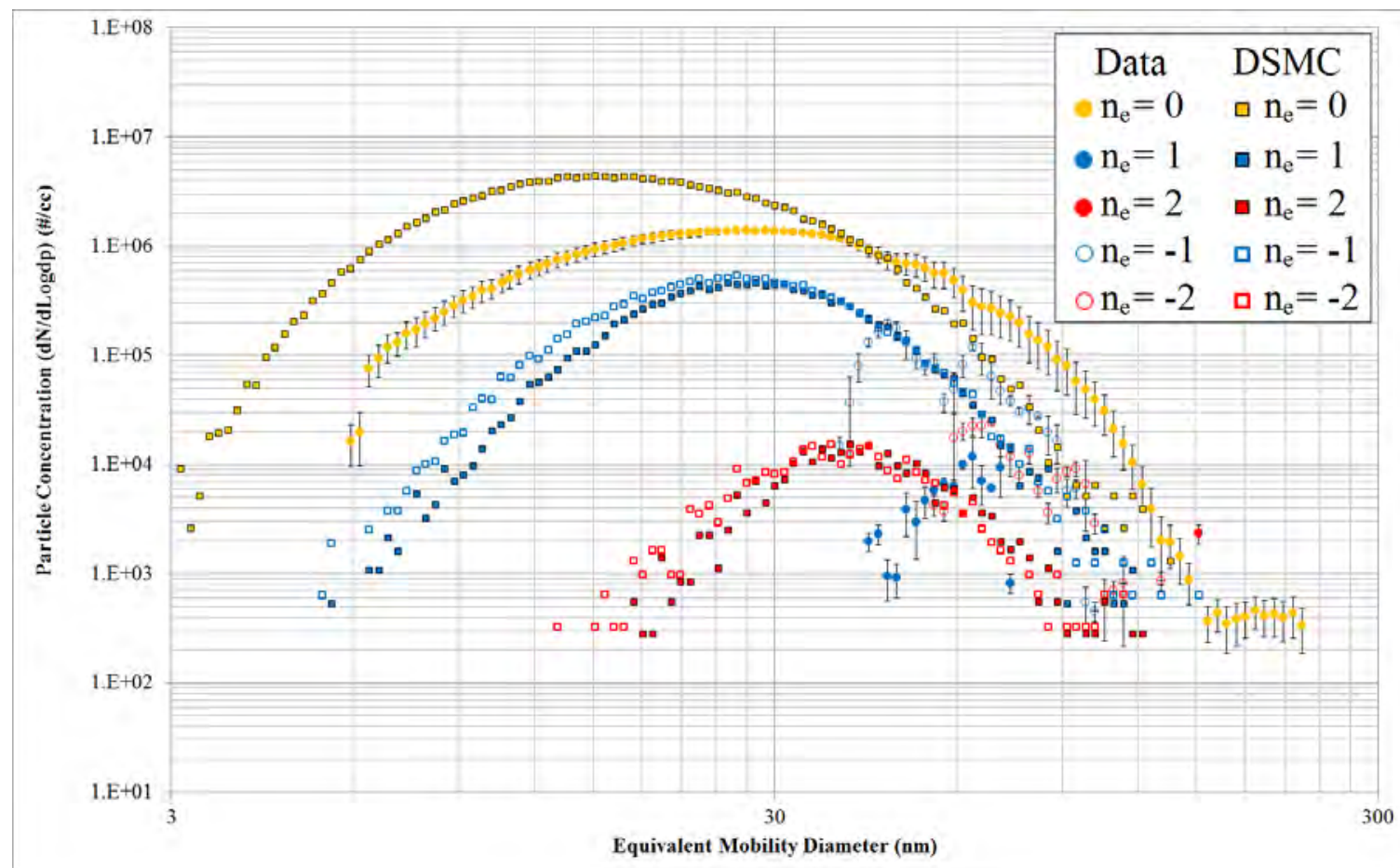

Figure 31. Comparison between the measured size and charge distribution for spark generated silver nanoparticles at coagulation chamber sampling port \#5 ( $\mathrm{t}=392.8 \mathrm{sec})$ and the coagulation model using the DSMC method. 


\subsubsection{Carbon}

The results for carbon show that initially all charge levels are over predicted by the DSMC model at Port \#2. Deviations between model and measurement are then less dramatic at Port \#3 which shows fair agreement with positively charged particle distributions and an under prediction of negatively charged particle distributions. At Port \#4 fair agreement between model and measurement is maintained for the neutral particle distribution, while for charged particle distributions the model over predicts measurements for positively charged particles and under predicts results for negatively charged particles. DSMC results at Port \#5 continue to show the deviations observed at Port \#4 and, in addition, the predicted particle distributions are shifted toward smaller diameters indicating that there are additional mechanisms not accounted for which lead to enhanced coagulation rates. 


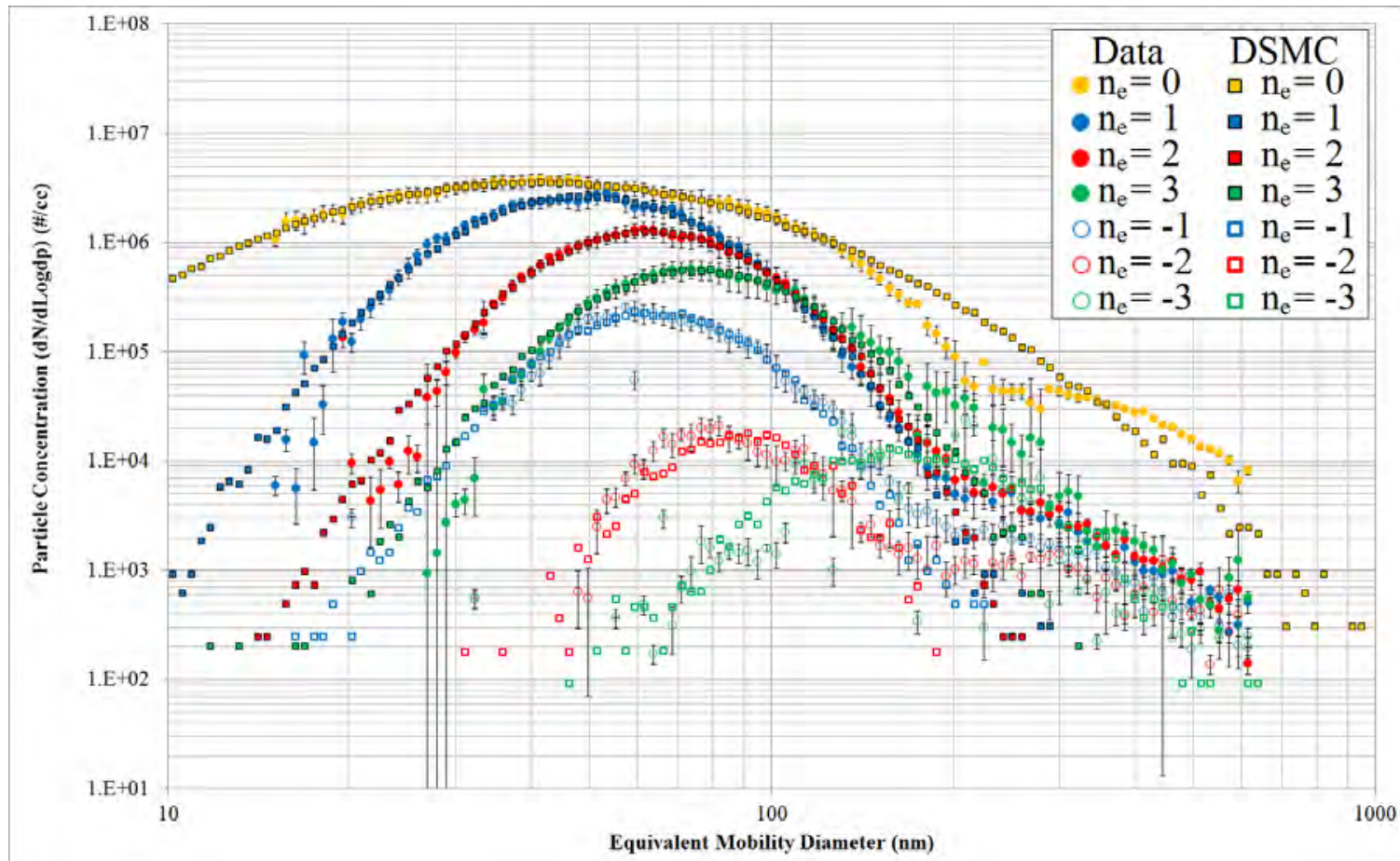

Figure 32. Comparison between the measured size and charge distribution for spark generated carbon nanoparticles at coagulation chamber sampling port \#1 ( $\mathrm{t}=0 \mathrm{sec})$ and the coagulation model using the DSMC method.

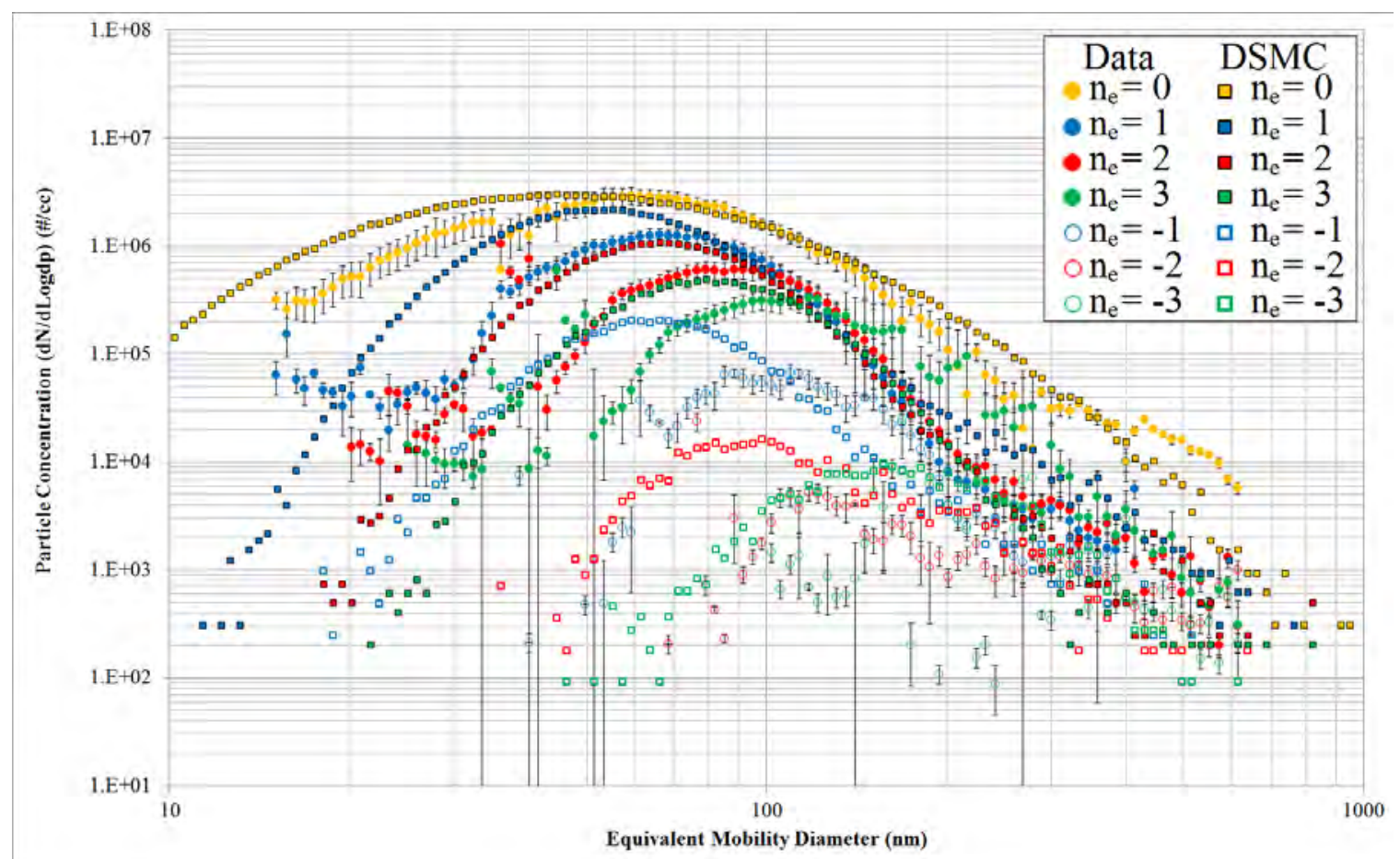

Figure 33. Comparison between the measured size and charge distribution for spark generated carbon nanoparticles at coagulation chamber sampling port \#2 $(\mathrm{t}=26.2 \mathrm{sec})$ and the coagulation model using the DSMC method. 


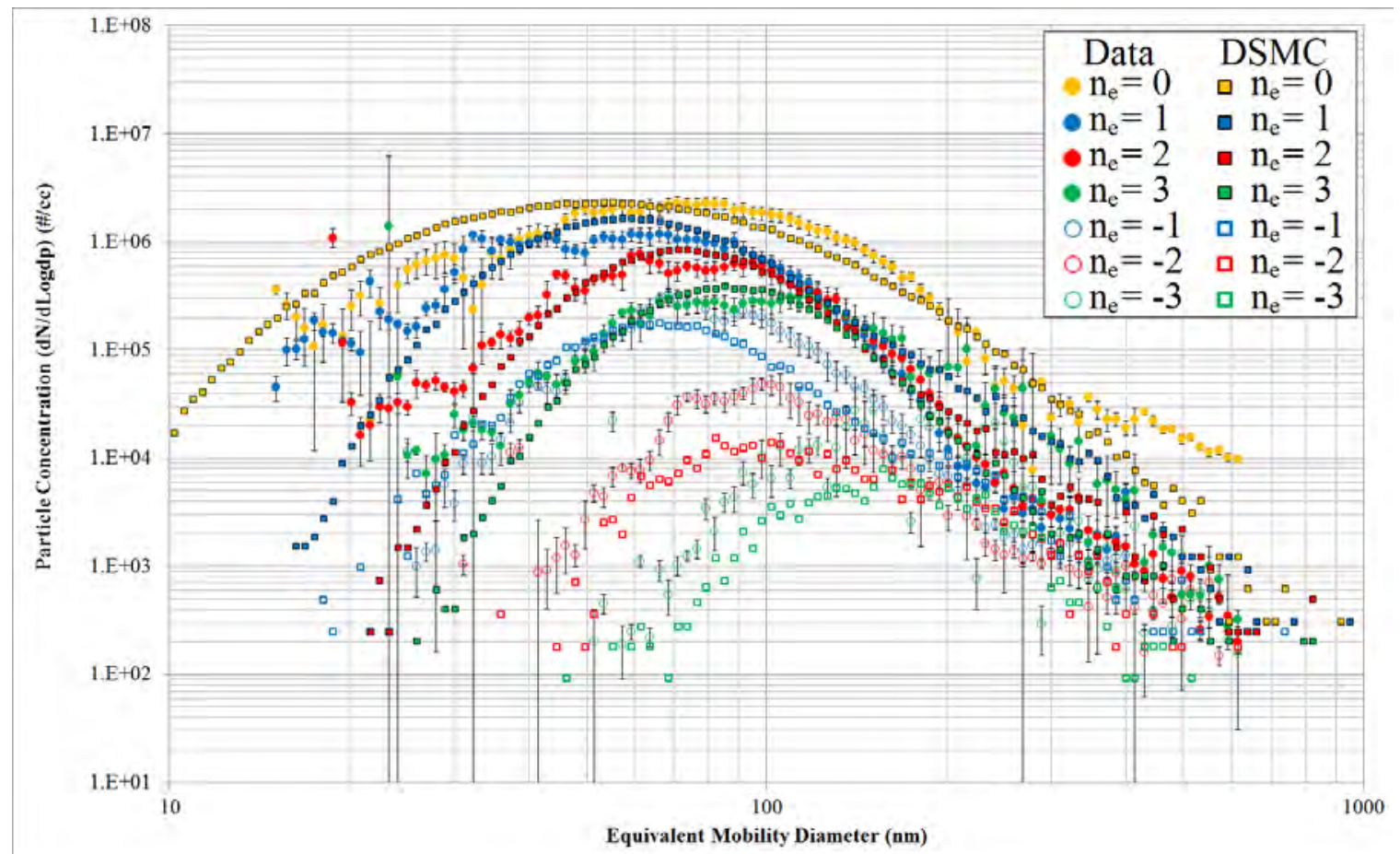

Figure 34. Comparison between the measured size and charge distribution for spark generated carbon nanoparticles at coagulation chamber sampling port \#3 $(\mathrm{t}=78.6 \mathrm{sec})$ and the coagulation model using the DSMC method.

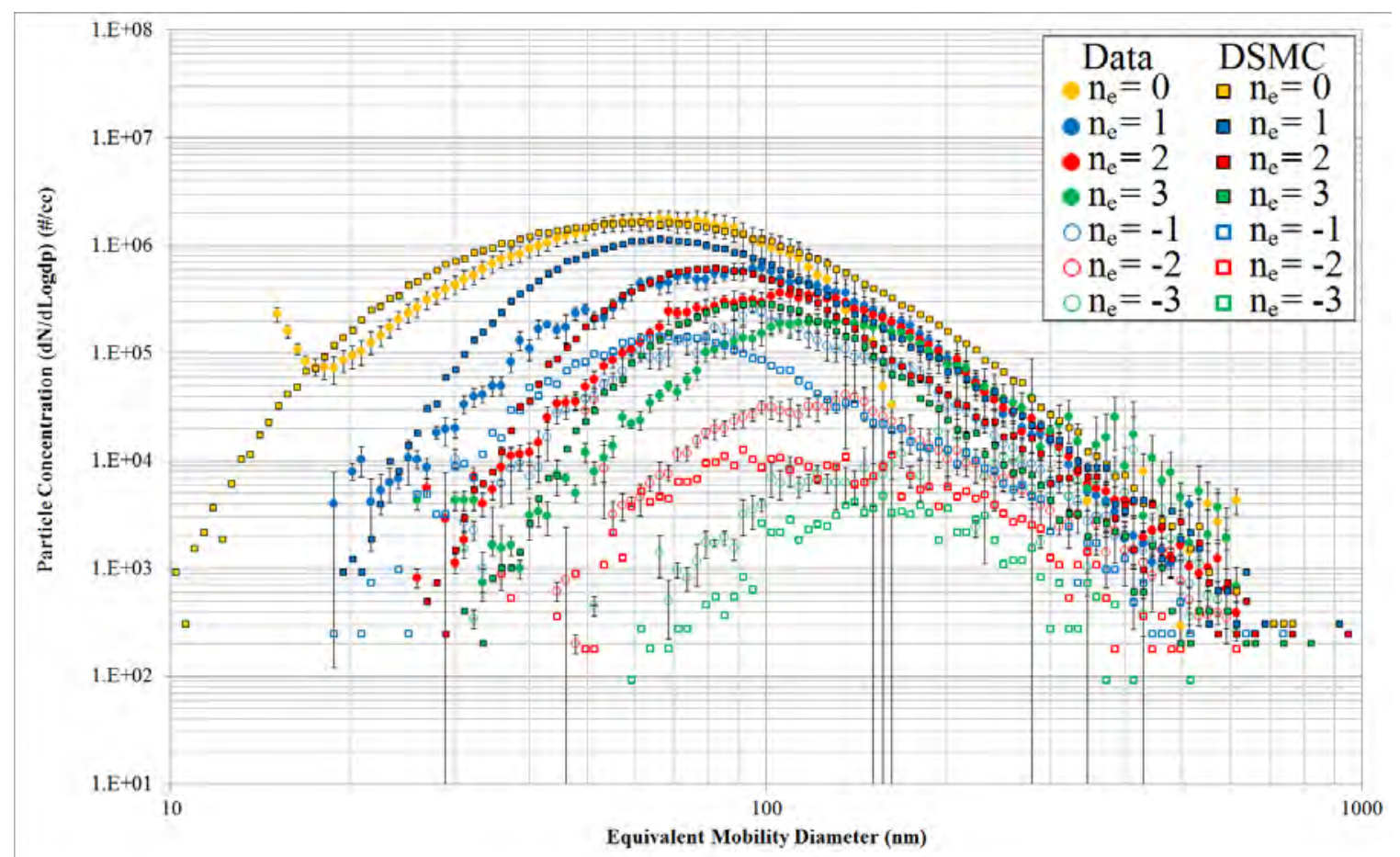

Figure 35. Comparison between the measured size and charge distribution for spark generated carbon nanoparticles at coagulation chamber sampling port \#4 ( $\mathrm{t}=183.3 \mathrm{sec})$ and the coagulation model using the DSMC method. 


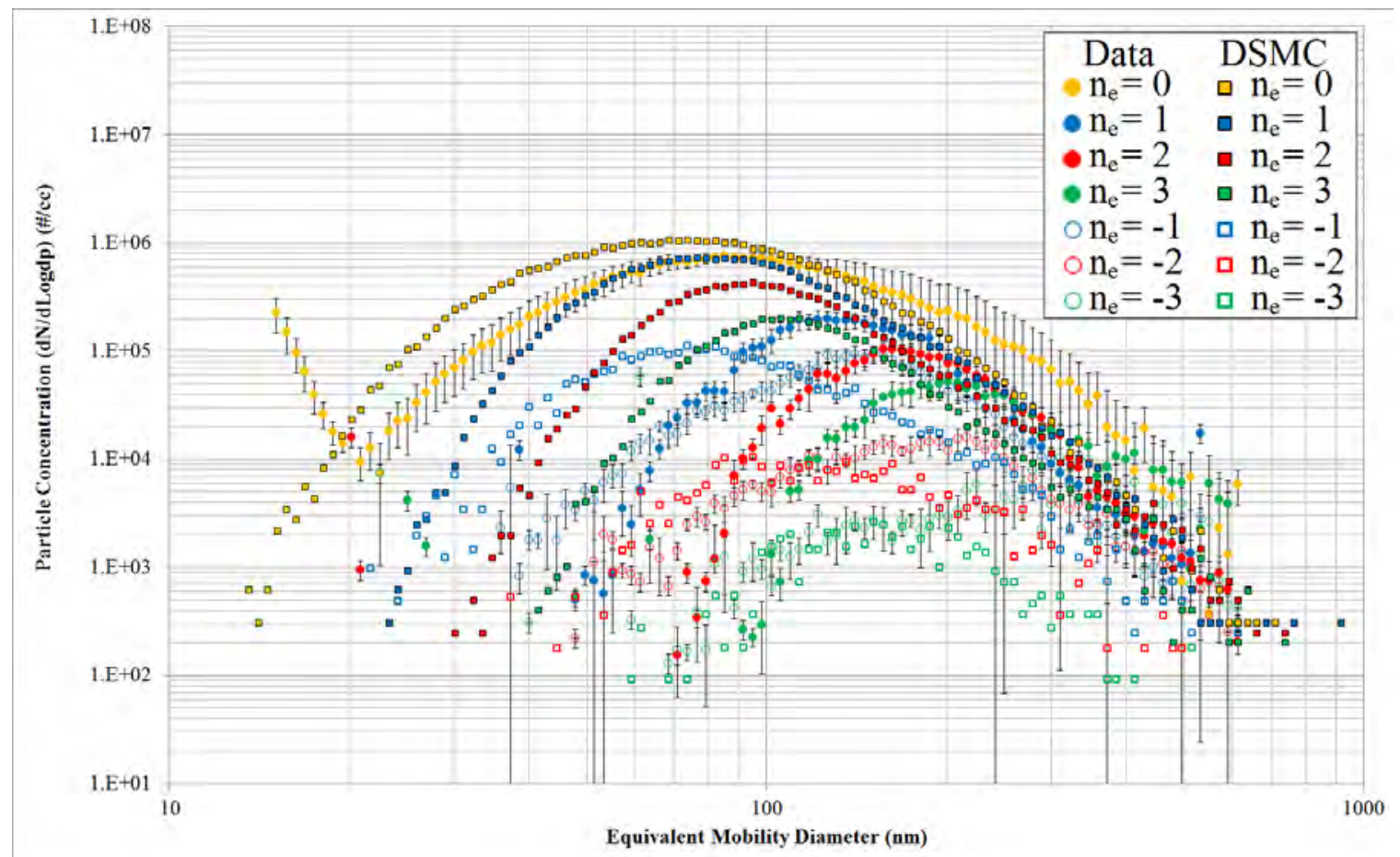

Figure 36. Comparison between the measured size and charge distribution for spark generated carbon nanoparticles at coagulation chamber sampling port \#5 ( $\mathrm{t}=392.8 \mathrm{sec})$ and the coagulation model using the DSMC method. 


\section{Chapter 5}

\section{DISCUSSION AND CONCLUSION}

The TDMA has been used here to investigate the coagulation of charged, polydisperse silver and carbon aerosols for comparison with computer models. The TDMA was made up of a TSI 3080 Electrostatic Classifier and a TSI 3936 Scanning Mobility Particle Sizer

Spectrometer connected in series, and a custom LabVIEW ${ }^{\circledR}$ program has been created to automate the voltage stepping process and expedite data collection. A new TDMA inversion algorithm is presented based on the least squares method which has been verified against a charged neutralized aerosol with a known charge distribution.

An open flow chamber has also been designed and constructed for aerosol coagulation studies. The chamber is supplied with a test aerosol which first flows into a large angle diffuser used to expand and slow down the flow to increase the residence time of the aerosol within the chamber. The diffuser was carefully designed with deflectors to provide an effective wall expansion angle of $7^{\circ}$ between plates to prevent flow recirculation while obtaining a total wall expansion angle of $22.5^{\circ}$. The aerosol is sampled from one of five sampling ports positioned axially along the chamber wall while the other four sampling ports are covered to prevent flow disturbances and pressure loss. Throughout the experiment the sampling probe is placed at all five sampling ports to observe the change in the aerosol size and charge distribution over time.

Differences in the charge state of particles measured at Port \#1 were observed with the silver particles being bipolar and carbon showing strong charge asymmetry with 
a higher concentration of positively charged particles. TEM images of silver and carbon nanoparticles produced by the spark generator show that the silver particles are nearly spherical while carbon particles form complex chain agglomerates. The agglomerated particles may have a lower neutralization rate due to screening (Kim et al. 2005) which would partially explain the charge asymmetry.

The uncertainty in the TDMA results is quite high in some cases and the distributions themselves are not always smooth as expected. Since the distributions obtained during TDMA calibration with a vibrating mesh nebulizer were found to be smooth, it can be said that the constancy of aerosol generation during measurements is a major factor. In this case only repeated measurements will improve the measurement uncertainty from the spark generator. A small amount of very large errors exceeding $100 \%$ were also found in all measured distributions. These cases are traced back to the inversion process and the least squares method where the coefficient matrix contains two or more coupled charge levels. Since the number concentration of particles at different charge levels, at constant diameter, can be separated by an order of magnitude or more, the objective function minimized during the least squares process will be biased toward lower charge levels. From Eqn. 21 the uncertainty is proportional to the square root of the objective function which will then place large uncertainty on higher charge levels. As an example consider the sample data below for carbon measured at Port \#1 at size bin $d_{p, i}=27.9 \mathrm{~nm}:$ 


$\left[\begin{array}{c}140.145 \\ 277.351 \\ 417.035 \\ 162.789 \\ 852.51 \\ 3080.4 \\ 5868.77 \\ 6548.33 \\ 4691.02 \\ 19801.9 \\ 70377.1 \\ 173885 \\ 209971 \\ 125259 \\ 53170.1\end{array}\right]=\left[\begin{array}{ccc}0 & 0 & 0.387559 \\ 0 & 0 & 0.330435 \\ 0 & 0.00191655 & 0.00186031 \\ 0 & 0.0351436 & 0 \\ 0 & 0.20803 & 0 \\ 0 & 0.418587 & 0 \\ 0 & 0.259749 & 0 \\ 0 & 0.169525 & 0 \\ 0 & 0.0125805 & 0 \\ 0.00816096 & 0 & 0 \\ 0.0881642 & 0 & 0 \\ 0.323481 & 0 & 0 \\ 0.398964 & 0 & 0 \\ 0.140759 & 0 & 0 \\ 0.00908483 & 0 & 0\end{array}\right]\left[\begin{array}{l}n_{0}\left(d_{p, i}, 1\right) \\ n_{0}\left(d_{p, i}, 2\right) \\ n_{0}\left(d_{p, i}, 3\right)\end{array}\right]$

Matrix $A$ is first decoupled, in this case charge levels $2 \& 3$ remain coupled,

$\left[\begin{array}{c}19801.9 \\ 70377.1 \\ 173885 \\ 209971 \\ 125259 \\ 53170.1\end{array}\right]=\left[\begin{array}{c}0.00816096 \\ 0.0881642 \\ 0.323481 \\ 0.398964 \\ 0.140759 \\ 0.00908483\end{array}\right]\left[n_{0}\left(d_{p, i}, 1\right)\right]$

$\left[\begin{array}{c}140.145 \\ 277.351 \\ 417.035 \\ 162.789 \\ 852.51 \\ 3080.4 \\ 5868.77 \\ 6548.33 \\ 4691.02\end{array}\right]=\left[\begin{array}{cc}0 & 0.387559 \\ 0 & 0.330435 \\ 0.00191655 & 0.00186031 \\ 0.0351436 & 0 \\ 0.20803 & 0 \\ 0.418587 & 0 \\ 0.259749 & 0 \\ 0.1695525 & 0 \\ 0.0125805 & 0\end{array}\right]\left[\begin{array}{l}n_{0}\left(d_{p, i}, 2\right) \\ n_{0}\left(d_{p, i}, 3\right)\end{array}\right]$


The least squares results are presented below in Table 4. Since the coupling rows seem to make up a small number of the total rows, it may be worthwhile investigating the effects of removing this row from the data set to fully decouple all charge levels. This type of error could also be reduced in the future by increasing the number of repeated measurements and by increasing the mobility resolution during the stepping process to obtain better statistical results in the least squares process.

Table 4. Least squares results for TDMA data collected for positively charged spark generated carbon nanoparticles at size bin $d_{p, i}=27.9 \mathrm{~nm}$.

\begin{tabular}{clll}
\hline \hline & $n_{e, k}=1$ & $n_{e, k}=2$ & $n_{e, k}=3$ \\
\hline$n_{0}\left(d_{p, i}, n_{e, k}\right)(\# / \mathrm{cc})$ & 541522 & 21655 & 726 \\
$S_{i}$ & $4.737 \times 10^{14}$ & $2.679 \times 10^{11}$ & $2.679 \times 10^{11}$ \\
$C_{i, k k}^{-1}$ & 0.00001830 & 0.0008608 & 0.01948 \\
$\sigma n_{0}\left(d_{p, i}, n_{e, k}\right)(\# / \mathrm{cc})$ & 41638 & 5740 & 27307 \\
Rel. error $(\%)$ & 7.69 & 26.5 & 3762 \\
\hline \hline
\end{tabular}

The results for both silver and carbon aerosol indicate that coagulation occurs faster than predicted by the model, at times differing by an order of magnitude. Model assumptions included Brownian coalescence of spherical particles with charge effects accounted for using the Fuchs correction for Coulombic interaction between charged particles. In the case of spark generated carbon the complexity of particle shape is significant. Similar structures are expected in HTGR graphite particles and therefore shape effects will be important for future source terms calculations for HTGRs. In addition, this model completely neglects the presence of background ions, which in this case may be elevated above normal atmospheric levels due to the electrical discharge process used to create the aerosol. Background ions play an important role in the charging of an aerosol and will affect both the final charge state and the rate at which the 
final charge state is achieved (Soo 1971; Whitby and Liu 1966; Williams and Loyalka 1991). Since the aerosol was measured immediately after generation, a state of charge equilibrium may not have existed at Port \#1. In this case, diffusion charging of ions would have continued downstream alongside the coagulation process. Future experiments might ensure charge equilibrium by using a charge neutralizer so that ion-particle effects are small and a true bipolar state is achieved (see Alonso et al. 2001) for a discussion of the after-charging effects downstream of a charge neutralizer). Alternatively, ion concentrations could be quantified (possibly with a Langmuir probe) so that ion-particle interactions can be included within the mathematical model (Kim et al. 2005). With regards to HTGRs, the background ion concentration will also be enhanced due to the presence of background radiation and radioactive particles. Therefore inclusion of the ion population would also be beneficial for HTGR source term calculations.

Scan time is a major weakness of the TDMA method and since no alternative techniques for measuring the size and charge distribution of ultrafine particles are known to the author, improvements and optimization of the TDMA scanning process should be explored. A large reduction in scan time could be achieved by specifying the size range of the SMPS as a function of DMA-1 voltage. As TDMA measurements progress and the voltage on DMA-1 is increased, the particle size of singly charged particles is also increased. Therefore the lower size limit of the SMPS can also be increased over time to reduce the scan interval. Similarly, the upper size limit of the SMPS could also be adjusted with DMA-1 voltage since particle concentrations beyond a certain charge level will not be measureable. This results in effectively defining a size range as a function of particle mobility at each voltage step in the TDMA scan. Currently the AIM software 
does not permit scheduling a series of scans with varying size ranges. Overall, the apparatus developed here will support future coagulation studies of charged ultrafine aerosols at NSEI by providing data for validation of computer codes and guiding model development. 


\section{REFERENCES}

Alonso, M., Alguacil, F. J., Nomura, T. and Kousaka, Y. (2001). Examination of the after-charging effects downstream of an aerosol neutralizer. Journal of Aerosol Science 32:287-294.

Baron, P. A. and Willeke, K. (2001). Gas and Particle Motion, in Aerosol Measurement: Principles, Techniques, and Applications, P. A. Baron and K. Willeke, eds., John Wiley, New York, 61-97.

Bird, R. B., Stewart, W. E. and Lightfoot, E. N. (2007). Transport Phenomena, Revised 2nd ed. John Wiley \& Sons, Inc., New York.

Boddu, S. R., Gutti, V. R., Tushar, K. G., Tompson, R. V. and Loyalka, S. K. (2011). Gold, silver, and palladium nanoparticle/nano-agglomerate generation, collection, and characterization. Journal of Nanoparticle Research 13:6591-6601.

Brockmann, J. E. (2001). Sampling and Transport of Aerosols, in Aerosol Measurement: Principles, Techniques, and Applications, P. A. Baron and K. Willeke, eds., John Wiley, New York, 143-195.

Chadha, T. S., Chattopadhyay, S., Venkataraman, C. and Biswas, P. (2012). Study of the charge distribution of liposome particles aerosolized by air-jet atomization. Journal of Aerosol Medicine and Pulmonary Drug Delivery 25:355-364.

Cheng, D. Y. (1992). Large Angle Diffuser Diverter Design for Maximum Pressure Recovery. U.S. Patent No. 5,165,452. Washington, DC: U.S. Patent and Trademark Office.

Cheng, Y.-S. (2001). Condensation Detection and Diffusion Size Separation Techniques, in Aerosol Measurement: Principles, Techniques, and Applications, P. A. Baron and K. Willeke, eds., John Wiley, New York, 569-601.

Clement, C. F., Clement, R. A. and Harrison, R. G. (1995). Charge distributions and coagulation of radioactive aerosols. Journal of Aerosol Science 26:1207-1225.

Covert, D., Wiedensohler, A. and Russel, L. (1997). Particle charging and transmission efficiencies of aerosol charge neutralizers. Aerosol Science and Technology 27:206-214.

Davies, C. N. (1979). Coagulation of aerosols by Brownian motion. Journal of Aerosol Science 10:151-161.

Devir (Wienstock), S. E. (1967). On the coagulation of aerosols III. Effect of weak electric charges on rate. Journal of Colloid and Interface Science 21:80-89. 
Flagan, R. C. (2001). Electrical Techniques, in Aerosol Measurement: Principles, Techniques, and Applications, P. A. Baron and K. Willeke, eds., John Wiley, New York, 537-568.

Friedlander, S. K. (2000). Smoke, Dust, and Haze: Fundamentals of Aerosol Dynamics. Oxford University Press, New York.

Fuchs, N. A. (1964). The Mechanics of Aerosols. Pergamon Press, Oxford.

Gelbard, F. (1982). MAEROS User Manual, Division of Reactor Safety Research,Office of Nuclear Regulatory Research, U. S. Nuclear Regulatory Commision, Sandia National Laboratory, Albuquerque, NM (NUREG/CR-1391, SAND80-0822).

Gensdarmes, F., Boulaud, D. and Renoux, A. (2001). Electrical charging of radioactive aerosols-Comparison of the Clement-Harrison models with new experiments. Journal of Aerosol Science 32:1437-1458.

Gillespie, T. and Langstroth, G. O. (1952). Coagulation and deposition in still aerosols of various solids. Canadian Journal of Chemistry 30:1003-1011.

Gormley, P. G. and Kennedy, M. (1949). Diffusion from a stream flowing through a cylindrical tube. Proceedings of the Royal Irish Academy 52:163-169.

Gysel, M., McFiggans, G. B. and Coe, H. (2009). Inversion of tandem differential mobility analyzer (TDMA) measurements. Journal of Aerosol Science 40:134-151.

Hidy, G. M. and Brock, J. R. (1970). The Dynamics of Aerocolloidal Systems. Pergamon Press, Oxford.

Hounslow, M. J., Ryall, R. L. and Marshall, V. R. (1988). A discretized population balance for nucleation, growth, and aggregation. AIChE Journal 34:1821-1832.

Humrickhouse, P. W. (2011). HTGR Dust Safety Issues and Needs for Research and Development. INL/EXT-11-21097, Idaho National Laboratory, June 2011.

Incropera, F. P., DeWitt, D. P., Bergman, T. L. and Lavine, A. S. (2007). Fundamentals of Heat and Mass Transfer, 6th ed. John Wiley \& Sons, Inc., Hoboken, NJ.

Karlsson, M. N. A. and Martinsson, B. G. (2003). Methods to measure and predict the transfer function size dependence of individual DMAs. Journal of Aerosol Science 34:603-625.

Katzer, M., Weber, A. P. and Kasper, G. (2001). Collision kinetics and electrostatic dispersion of airborne submicrometer fractal agglomerates. Journal of Colloid and Interface Science 240:67-77.

Kennedy, H. (1916). The large ions and condensation-nuclei from flames. Proceedings of the Royal Irish Academy A 33:58-74. 
Kim, S. H., Woo, K. S., Liu, B. Y. H. and Zachariah, M. R. (2005). Method of measuring charge distribution of nanosized aerosols. Journal of Colloid and Interface Science 282:46-57.

Maisels, A., Kruis, F. E. and Fissan, H. (2002). Determination of coagulation coefficients and aggregation kinetics for charged aerosols. Journal of Colloid and Interface Science 255:332-340.

Maricq, M. M. (2005). The dynamics of electrically charged soot particles in a premixed ethylene flame. Combustion and Flame 141:406-416.

Maricq, M. M. (2006). A comparison of soot size and charge distributions from ethane, ethylene, acetylene, and benzene/ethylene premixed flames. Combustion and Flame 144:730-743.

Morris, R. N. (2008). Next Generation Nuclear Plant Phenomena Identification and Ranking Tables (PIRTs)-Volume 3: Fission-Product Transport and Dose PIRTs. NUREG/CR-6944, Vol. 3 (ORNL/TM-2007/147, Vol. 3), Oak Ridge National Laboratory, March 2008.

Palsmeier, J. F. and Loyalka, S. K. (2013). Evolution of Charged Aerosols: Role of Charge on Coagulation. Nuclear Technology 184:78-95.

Park, K., Dutcher, D., Emery, M., Pagels, J., Sakurai, H., Scheckman, J., Qian, S., Stolzenburg, M. R., Wang, X., Yang, J. and McMurry, P. H. (2008). Tandem measurements of aerosol properties - A review of mobility techniques with extensions. Aerosol Science and Technology 42:801-816.

Rader, D. J. and McMurry, P. H. (1986). Application of the tandem differential mobility analyzer to studies of droplet growth or evaporation. Journal of Aerosol Science 17:771787.

Rosinski, J., Werle, D. and Nagamoto, C. T. (1962). Coagulation and scavenging of radioactive aerosols. Journal of Colloid Science 17:703-716.

Shaw, D. T. (1978). Fundamentals of Aerosol Science. John Wiley \& Sons, Inc., New York.

Simones, M. P., Gutti, V. R., Meyer, R. M. and Loyalka, S. K. (2011). Measurements of aerosol charge and size distribution for graphite, gold, palladium, and silver nanoparticles. Nuclear Technology 176:211-226.

Simones, M. P., Loyalka, S. K., Power, P., Duffy, C., MacLoughlin, R. and Tatham, A. (Submitted). Measurement of the size and charge distribution of sodium chloride particles generated by an Aeroneb Pro ${ }^{\circledR}$ pharmaceutical nebulizer. European Journal of Nanomedicine. 
Soo, S. L. (1971). Dynamics of Charged Suspensions, in Topics in Current Aerosol Research Vol. 2, G. M. Hidy and J. R. Brock, eds., Pergamon Press, Oxford, 61-149.

Stolzenburg, M. R. (1988). An Ultrafine Aerosol Size Distribution Measuring System, Ph.D. Thesis. University of Minnesota.

Stolzenburg, M. R. and McMurry, P. H. (2008). Equations governing single and tandem DMA configurations and a new lognormal approximation to the transfer function. Aerosol Science and Technology 42:421-432.

Strang, G. (1980). Linear Algebra and its Applications. Academic Press, New York.

Subramanian, V., Baskaran, R. and Indira, R. (2008). Experimental study on enhanced Brownian coagulation of sodium compound aerosol in the presence of gamma field. Journal of Aerosol Science 39:814-818.

Vemury, S., Janzen, C. and Pratsinis, S. E. (1997). Coagulation of symmetric and asymmetric bipolar aerosols. Journal of Aerosol Science 28:599-611.

Vincent, J. H. (2007). Aerosol Sampling: Science, Standards, Instrumentation and Applications. John Wiley \& Sons, Inc., Hoboken, NJ.

Wang, S. C. and Flagan, R. C. (1990). Scanning electrical mobility spectrometer. Aerosol Science and Technology 13:230-240.

Whitby, K. T. and Liu, B. Y. H. (1966). The Electrical Behaviour of Aerosols, in Aerosol Science, C. N. Davies, ed., Academic Press, New York, NY, 59-86.

Williams, M. M. R. and Loyalka, S. K. (1991). Aerosol Science: Theory and Practice. Pergamon Press, Oxford.

Wolberg, J. (2006). Data Analysis Using the Method of Least Squares: Extracting the Most Information from Experiments. Springer-Verlag, Berlin, Germany.

Xiang, X. D. and Colbeck, I. (1993). Coagulation and wall loss of charged particles. Journal of Aerosol Science 24:S135-S136. 


\section{Appendix A \\ SMPS CALIBRATION}

The SMPS used in this study was calibrated for proper measurement of particle size by using a diluted solution of monodisperse polystyrene latex (PSL) spheres purchased from Bangs Laboratories, Inc. The solution was atomized using the system shown in Figure A1 consisting of a TSI 3076 Constant Output Atomizer (COA), a TSI 3062 Diffusion Dryer, and two additional diffusion dryers built in-house. The COA uses filtered compressed air from a pressurized cylinder (Airgas 'Zero' grade, $<3$ ppm $\mathrm{H}_{2} \mathrm{O}$ ) which is expanded through a horizontal orifice and over a vertical venturi. The increased air velocity over the venturi creates a low pressure region allowing the particle solution to be drawn from the bottle through a capillary tube and into the gas stream. As the solution enters the gas stream it is atomized forming a distribution of droplet sizes. Large droplets are separated by inertial impaction against the walls of the atomizer assembly and the remaining droplets follow the gas stream into the first diffusion dryer. All diffusion dryers function by passing the aerosol stream through a wire mesh tube. The outside of the tube is packed with a desiccant, such as silica gel or DRIERITE®, which allows the aerosol to flow unobstructed through the center of the dryer while allowing water vapor to diffuse and become adsorbed by the desiccant. A heating element wrapped around a stainless steel tube and powered by a variable autotransformer was used after the first diffusion dryer to promote further evaporation of water vapor from the aerosol particles which is then captured by the subsequent diffusion dryers. 
Using the aerosol generator previously described both $65 \mathrm{~nm}$ and $99 \mathrm{~nm}$ monodisperse aerosols were generated for instrument calibration. Each monodisperse solution was prepared by using $1000 \mathrm{ml}$ of $8 \mathrm{M} \Omega$ deionized water which was then incrementally concentrated with PSL using a micropipette while measuring the size distribution with the SMPS until a well-defined monodisperse peak could be acquired. Figure A2 below shows the size distributions measured for both the $65 \mathrm{~nm}$ and $99 \mathrm{~nm}$ PSL solutions and in each case a distinct peak was measured at the corresponding particle size bin. The remaining particles indicated in the measurements are likely due to the surfactant used to stabilize the PSL particles and possibly remaining water droplets. 


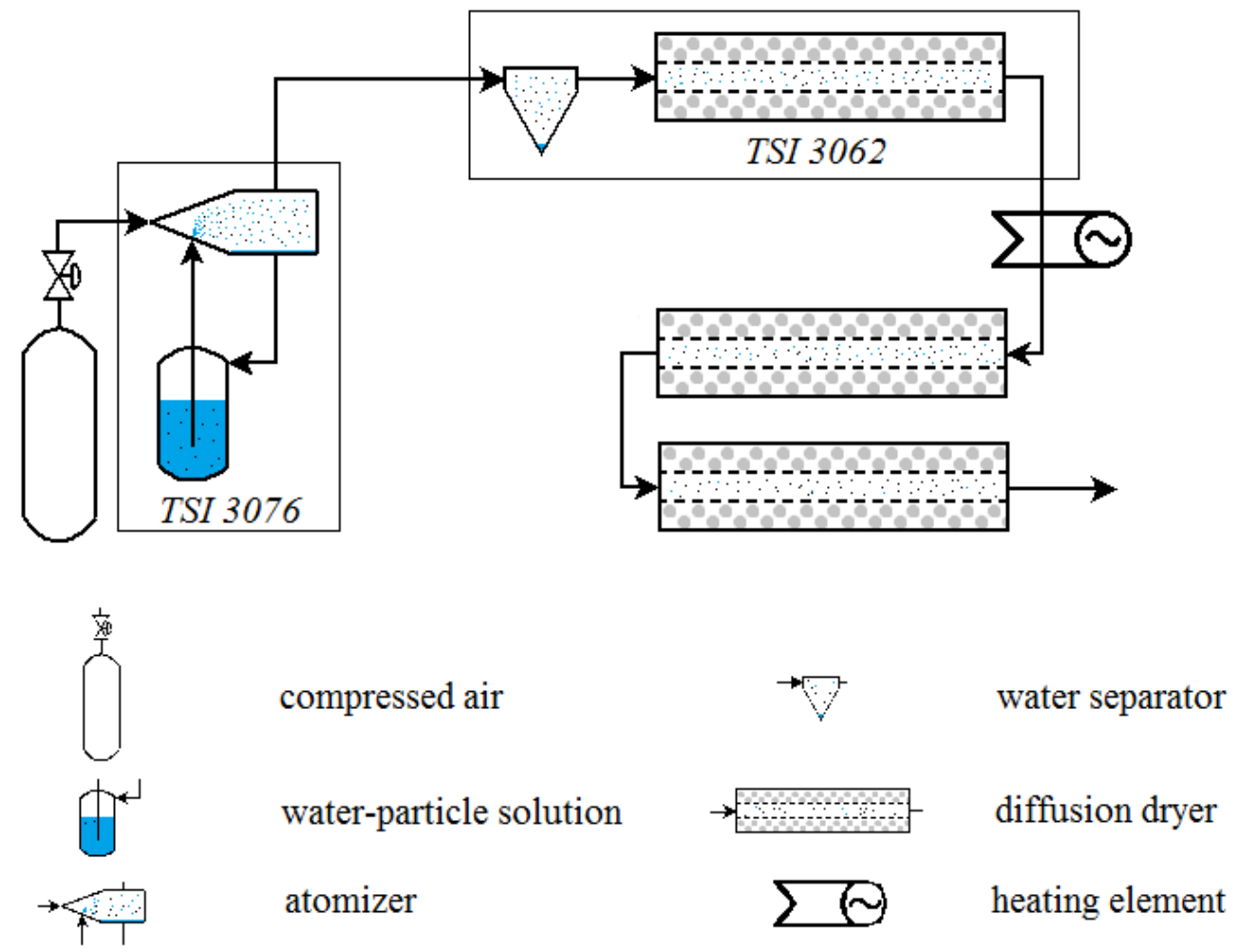

Figure A1. Aerosol generator for SMPS particle size calibration using aqueous solution of monodisperse particles. 

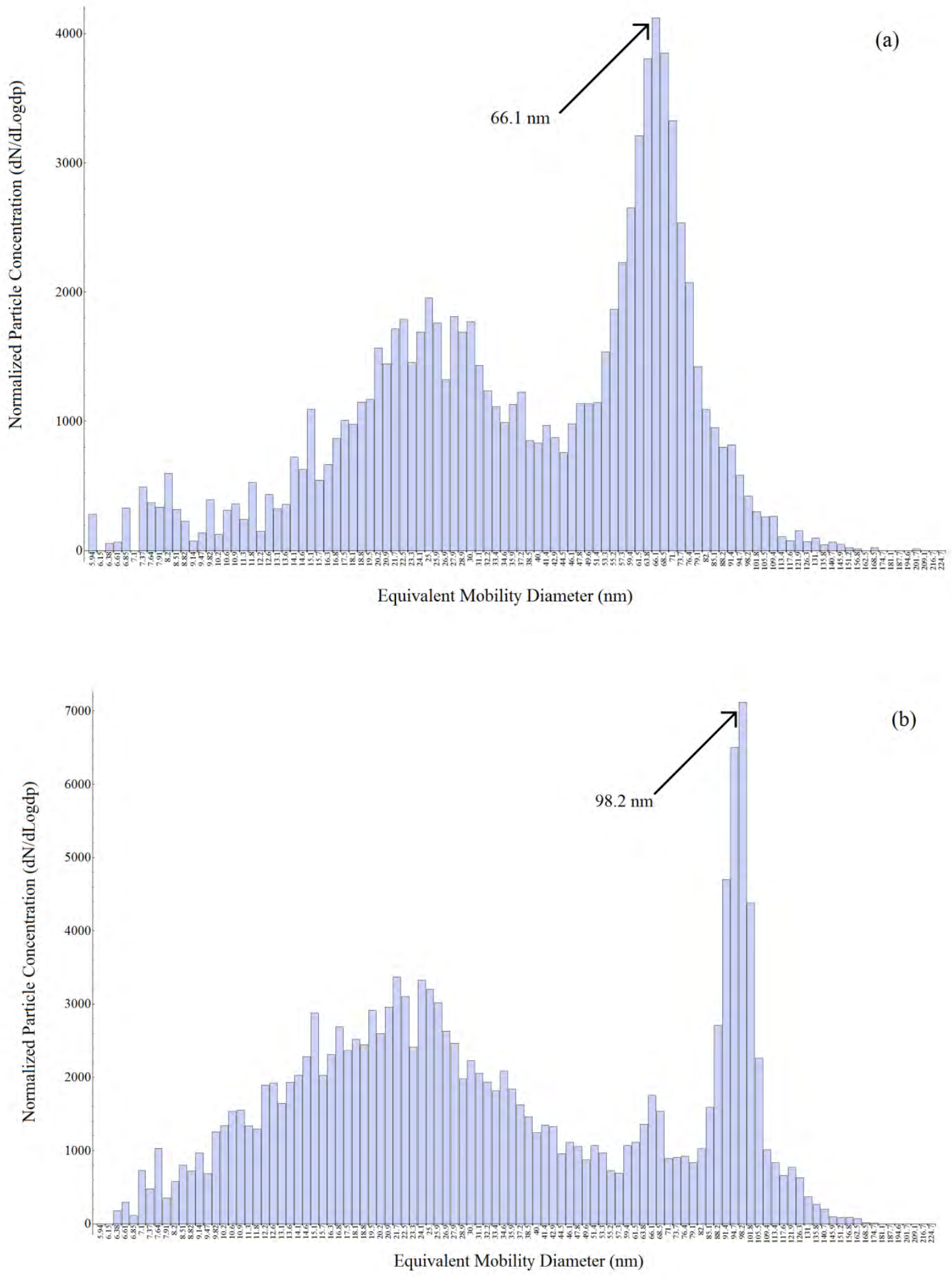

Figure A2. SMPS measurement of monodisperse PSL spheres of diameter (a) $65 \mathrm{~nm}$ and (b) $99 \mathrm{~nm}$. In each case the peak occurs within the size bin corresponding to the particular PSL solution used. 


\section{Appendix B \\ TDMA AUTOMATION USING LabVIEW®}

The TSI 3080 Electrostatic Classifier comes equipped from the manufacturer with a serial RS-232 interface option allowing the user to both send and receive commands from the instrument. LabVIEW ${ }^{\circledR}$ was used to take advantage of this option as a tool to monitor instrument status, adjust instrument settings, and control the voltage stepping of DMA-1.

The front panel of the LabVIEW ${ }^{\circ}$ program, shown in Figure B1, includes various gauges and displays for monitoring DMA-1 status including: sheath, bypass and impactor flow rates, sheath and cabinet temperatures, DMA pressure, pressure drop across impactor, and DMA voltage. In Addition, voltage and flow rate adjustments for DMA-1 can also be made from the front panel. LabVIEW® was also configured to monitor the analog input voltage signal for DMA-2 by using a BNC splitter and a data acquisition module (NI USB-9162 C Series USB Single Module Carrier with an NI 9219 24-Bit Universal Analog Input Module) which allowed the voltage stepping of DMA-1 to be automated, greatly expediting the measurement process. The LabVIEW® code is also provided as a reference in Figures B2-B8. 


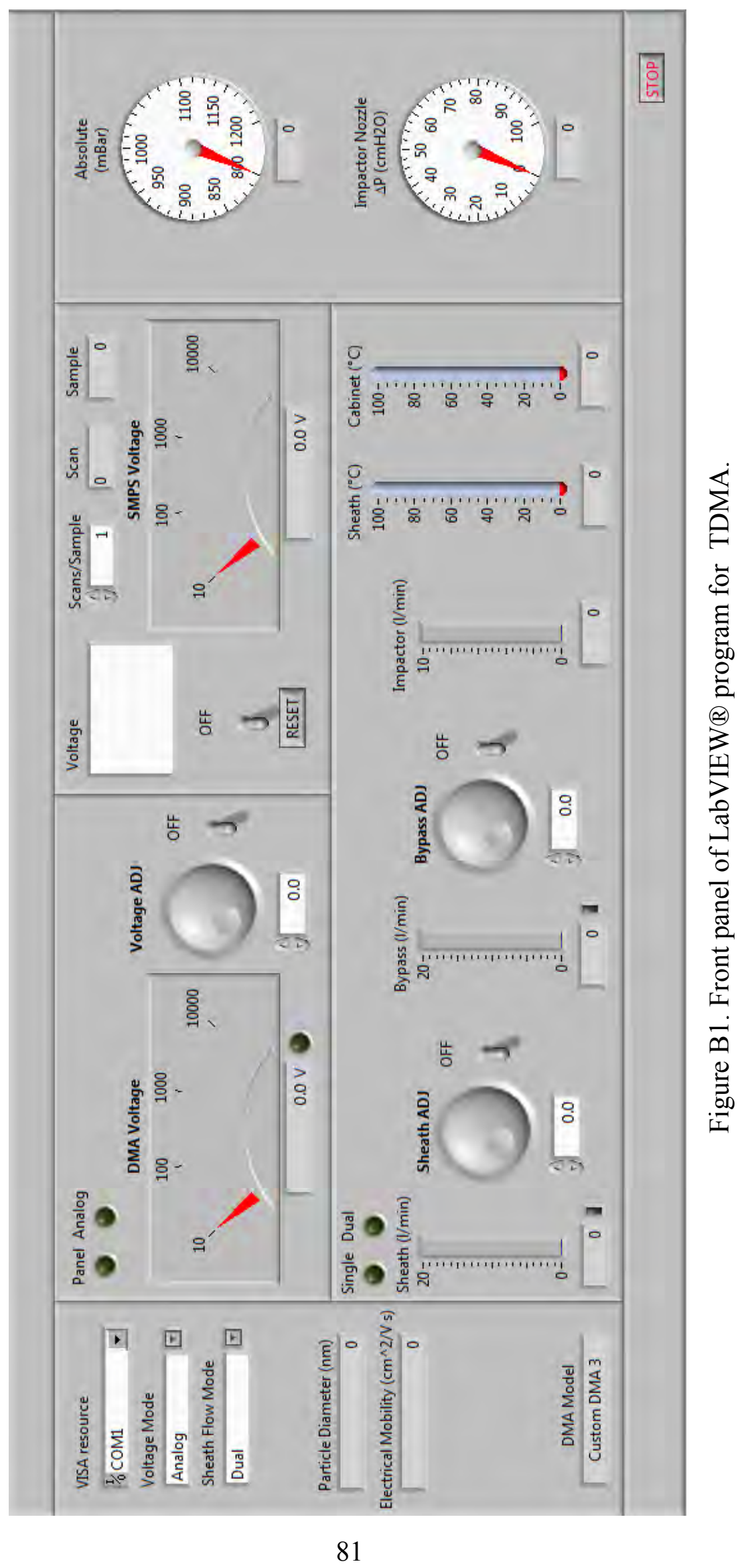




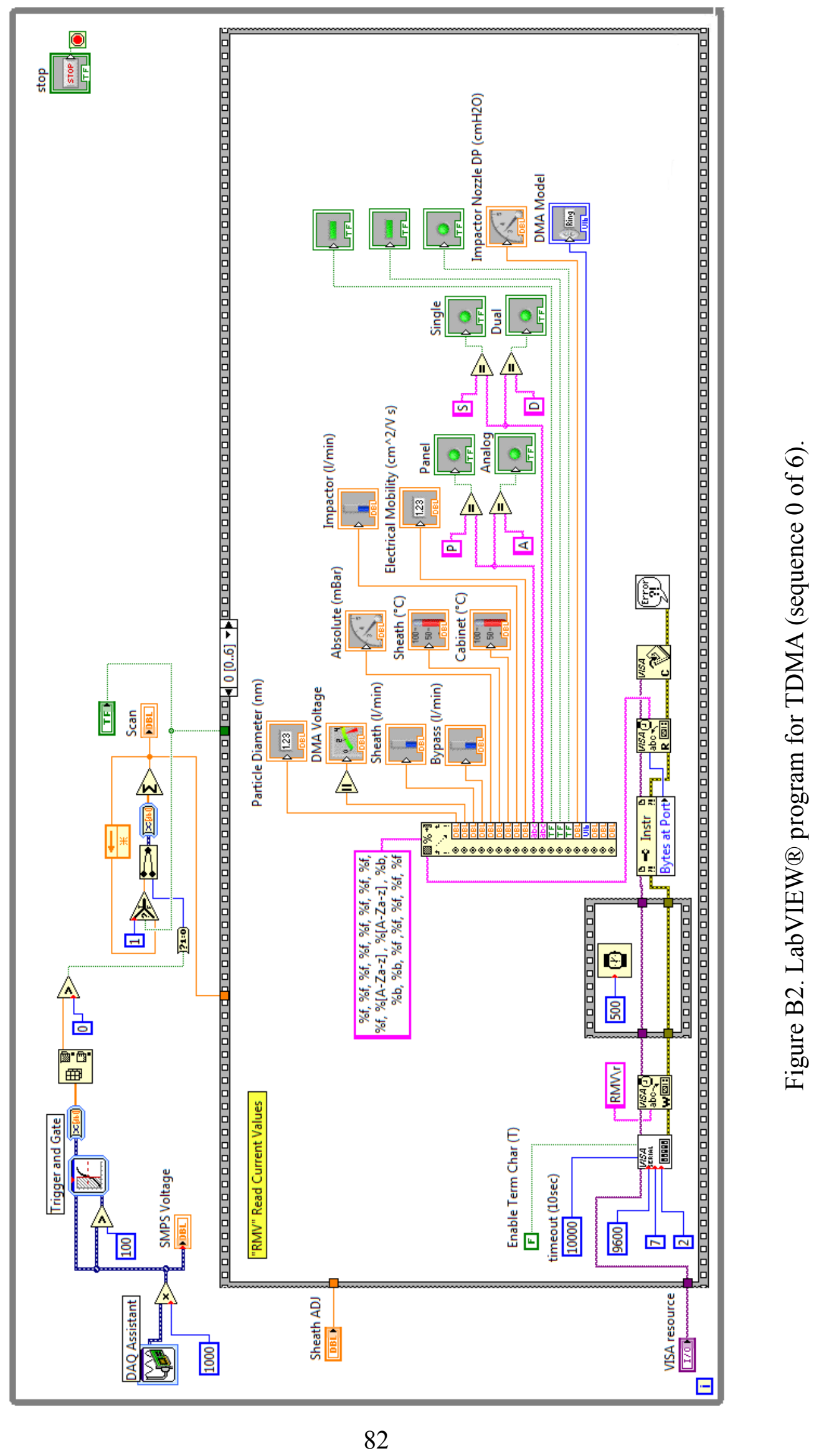




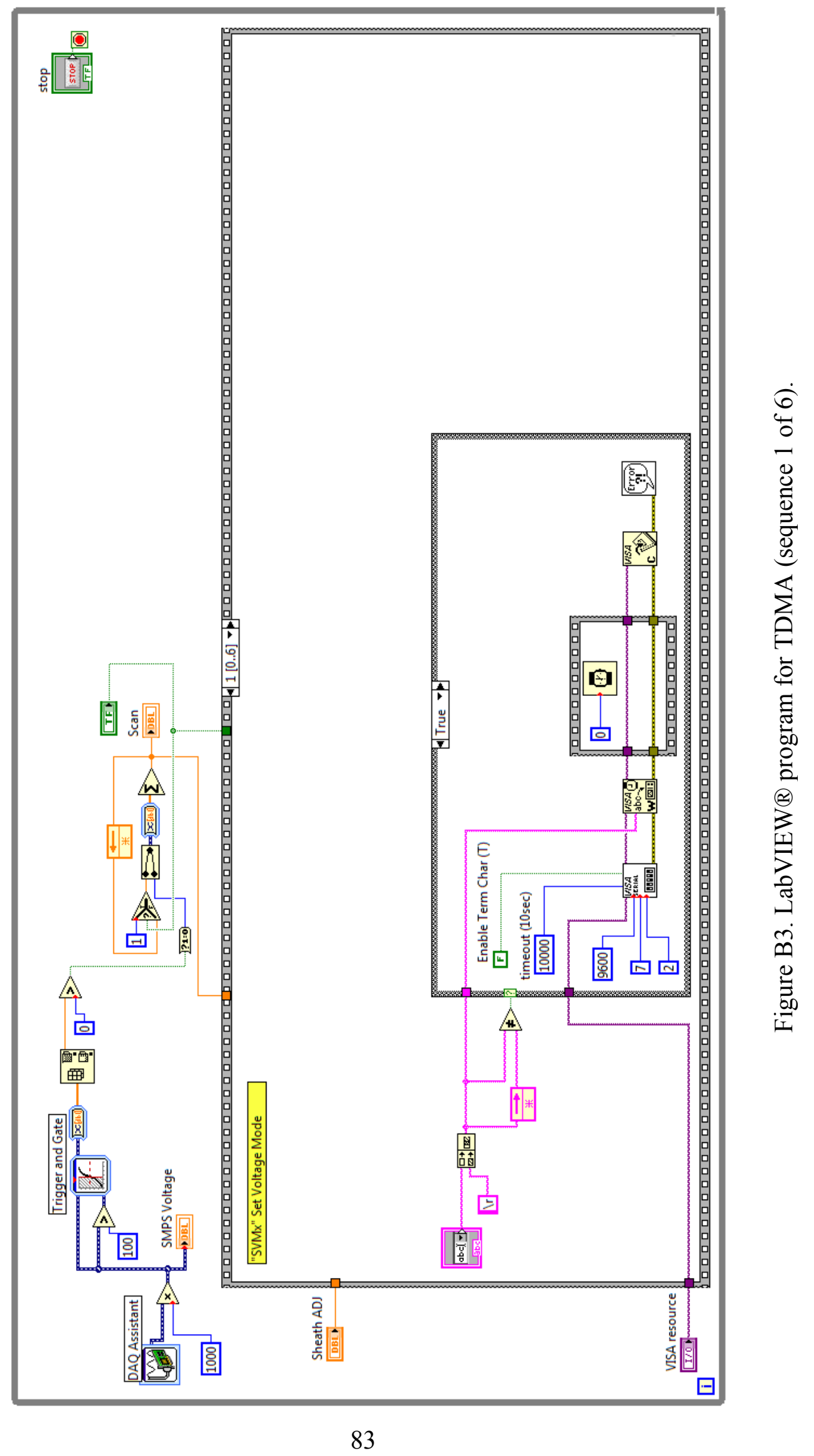




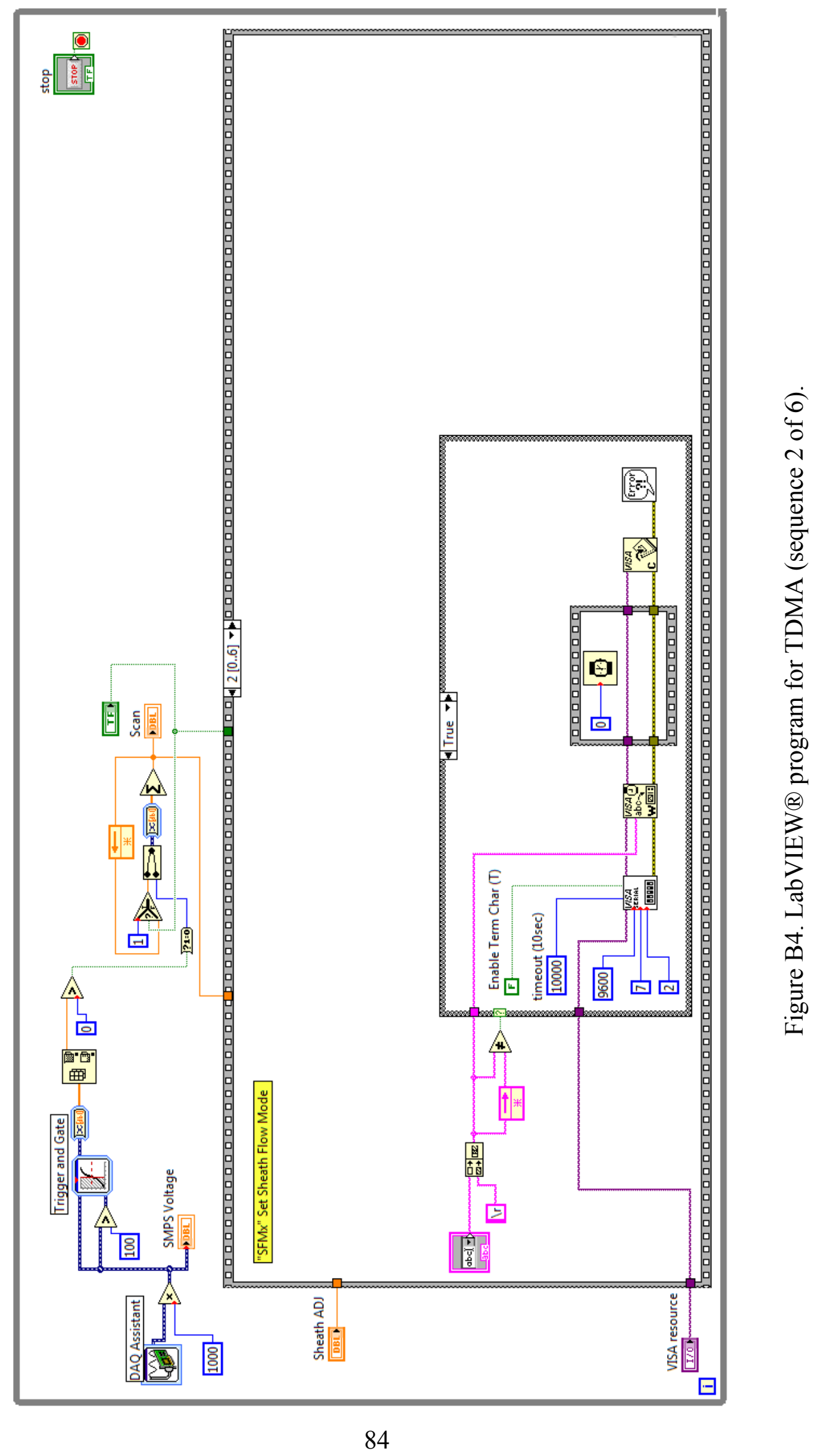




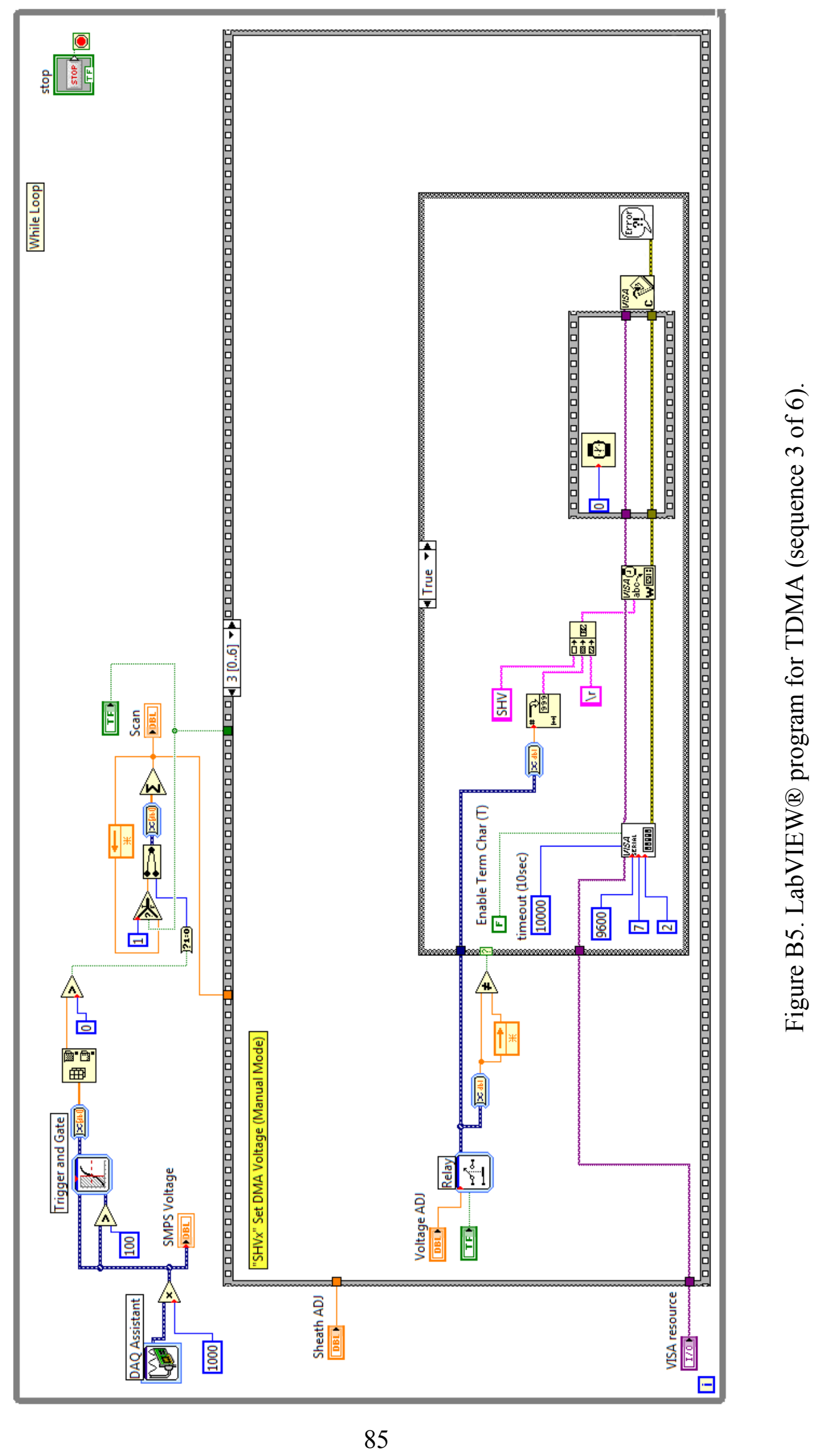




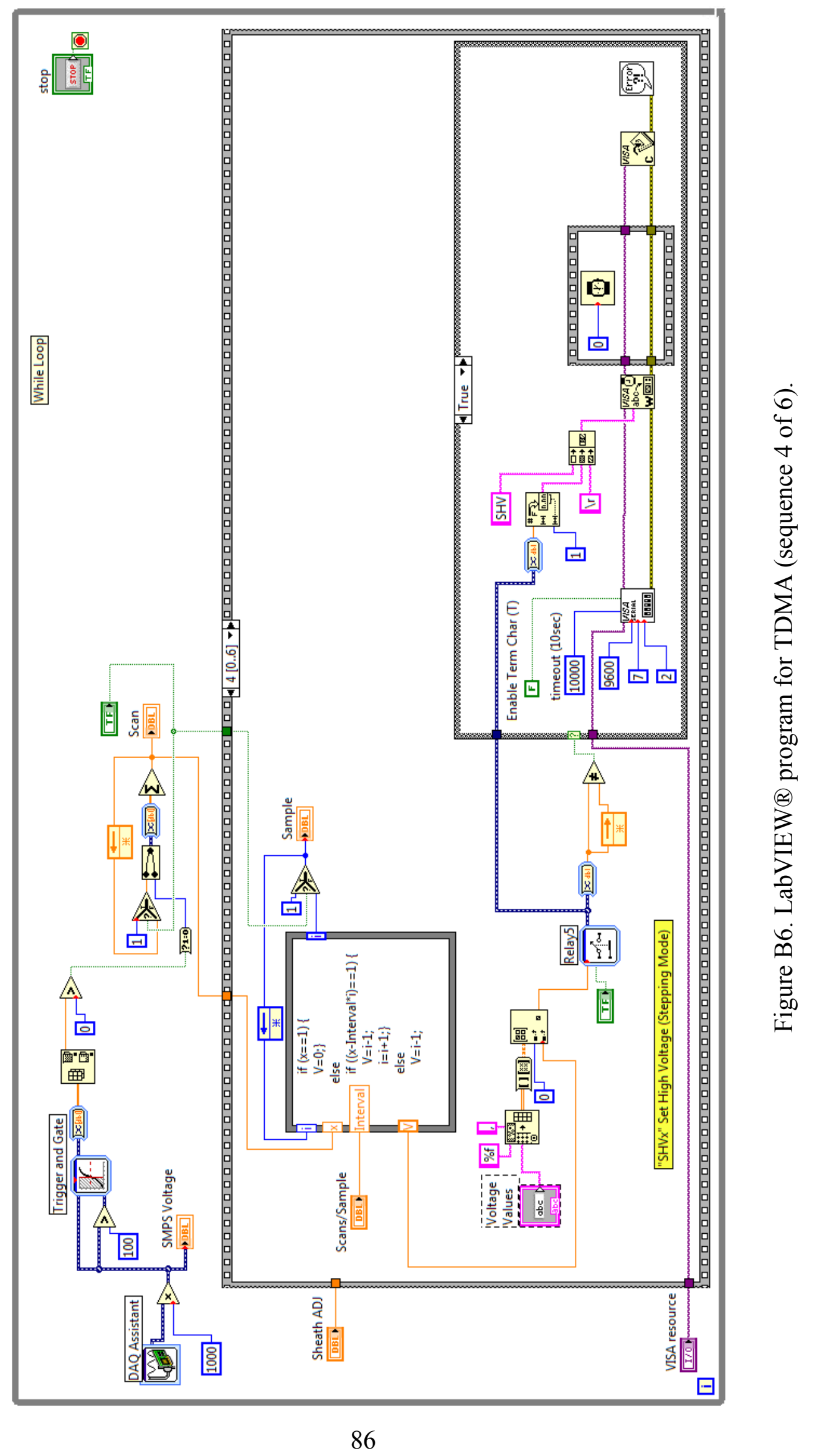




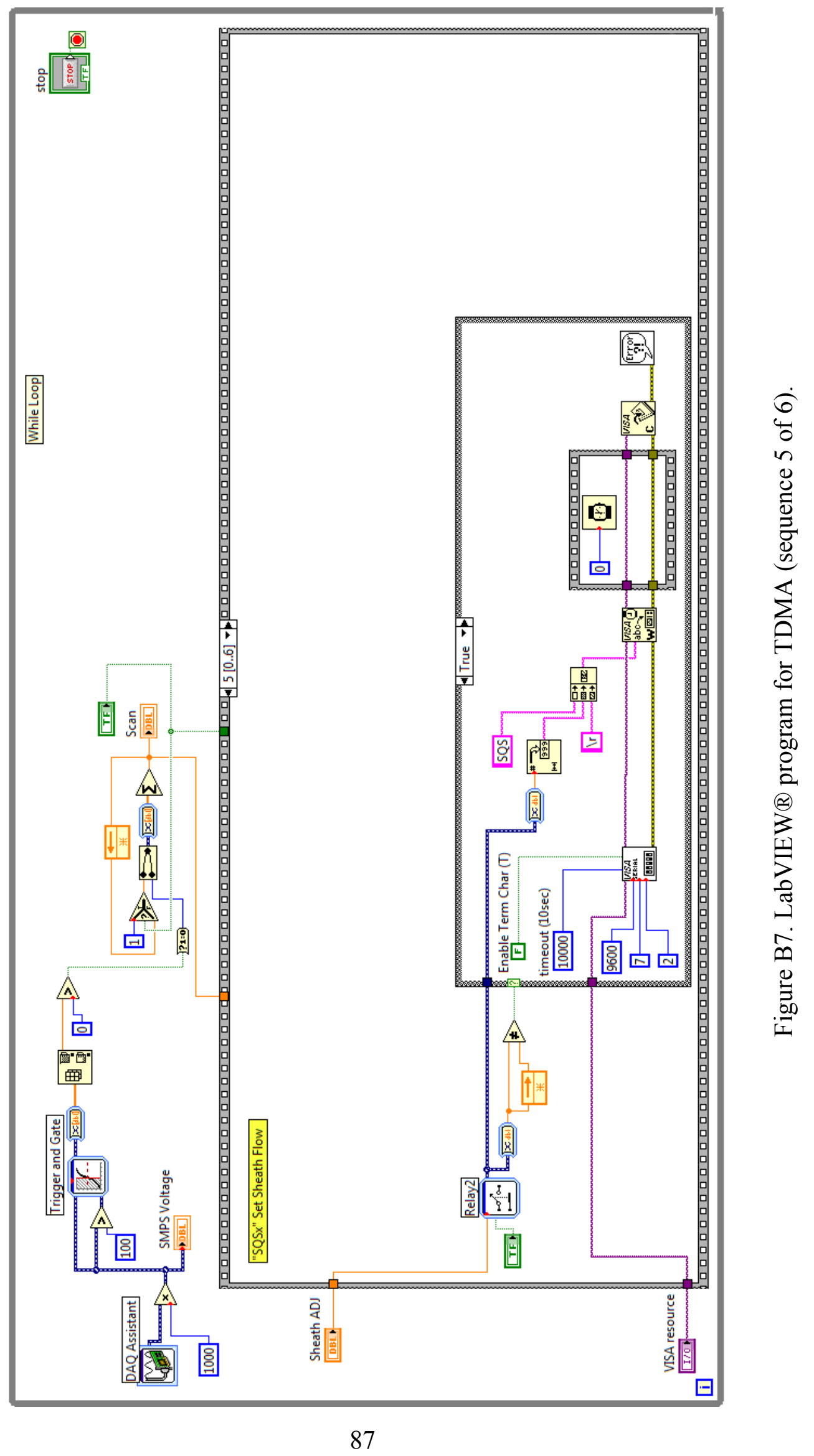




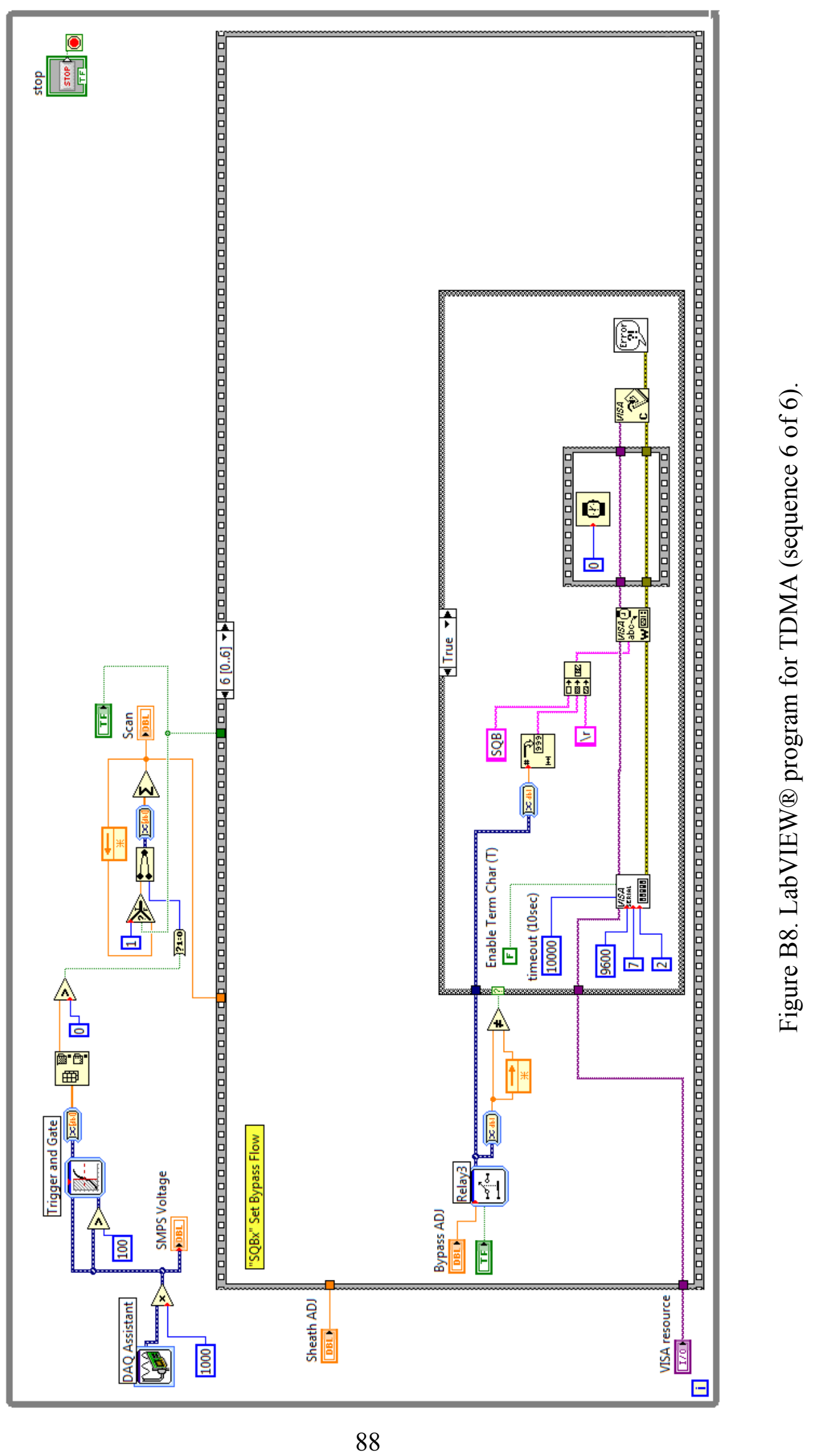




\section{Appendix C}

\section{TDMA CALIBRATION}

The TDMA used in this study was calibrated using sodium chloride aerosol generated from an Aeroneb Pro ${ }^{\circledR}$ vibrating mesh nebulizer as depicted in Figure C1. Airflow into the nebulizer setup was supplied via a diaphragm compressor (GAST DOL-101-AA) at a flow rate of $15 \mathrm{l} / \mathrm{min}$. The supplied air was dried using two laboratory drying units (DRIERITE \#26800) and filtered using a HEPA filter (TSI \#1602051). Using dry, filtered air provided a consistent air supply over long periods of time by preventing variations in humidity due to changing laboratory conditions which could otherwise lead to changes in droplet evaporation rates. Normal saline solution $(0.90 \% \mathrm{w} / \mathrm{v}$ of $\mathrm{NaCl})$ prepared using $\geq 99.5 \%$ sodium chloride (Fisher Scientific enzyme grade BP358) and $18 \mathrm{M} \Omega$ deionized water (Thermo Scientific NERL Reagent Grade) was used to produce salt nuclei aerosol. During operation the nebulized sample was transported by the clean airflow past a sharpedged, thin-wall sampling probe (1/4 in. OD) located $200 \mathrm{~mm}$ downstream from the nebulizer before being vented freely to the atmosphere. The sampled aerosol was immediately conditioned using a diffusion dryer to ensure that the nebulized droplets were completely evaporated to form single salt nuclei. Beyond the diffusion dryer the aerosol was transported, using conductive tubing, through a charge neutralizer and to either the TDMA or SMPS for characterization. Since theoretical charge distributions are represented in terms of charge fraction, the SMPS was used to obtain the total size distribution for normalization. Figure $\mathrm{C} 2$ shows the two experimental configurations used in this study in measuring the charge distributions for charged neutralized aerosols. 
As the SMPS repeatedly scanned from low to high voltage the LabVIEW® program monitored the SMPS voltage and adjusted the DMA-1 voltage when the end of a scan was detected. A delay of 200 seconds between SMPS scans allowed DMA-1 to reach equilibrium under the new voltage setting and for the new selection of equivalent electrical mobility particles to reach the SMPS. The process was repeated for a predetermined number of scans corresponding to the number of size bins measured by the SMPS. This process was repeated for both positively and negatively charged particles by switching the polarity of the high voltage module in DMA-1. Corrections for multiply charged particles and diffusion losses in the SMPS were also accounted for within the AIM software along with variations in temperature and pressure within DMA-2. Temperature and pressure values for DMA-1 were recorded manually at the start of each scan and corrections were carried out during data analysis within the Mathematica program. Corrections for diffusional losses within DMA-1 and transport tubing were accounted for during analysis using relations given by Karlsson and Martinsson (2003), and Friedlander (2000), respectively. A summary of instrument settings and parameters used are presented in Table C1.

The charge neutralized results shown in Figure C3 have been analyzed up to \pm 4 units of charge and show good agreement with the modified Fuchs model given by Wiedensohler (Flagan 2001). As predicted by the Fuchs model, the fraction of negatively charged particles exceeds the fraction of positively charged particles at all charge levels. However, the measured distributions do not indicate the magnitude in spread between polarities as predicted by Fuchs' theory and the fractions of positively and negatively charged particles at each charge level converge above $200 \mathrm{~nm}$. This convergence follows 
the Boltzmann equilibrium charge distribution model for $\pm 2, \pm 3$ and \pm 4 units of charge, while the fraction of singly charged particles above $200 \mathrm{~nm}$ falls off more rapidly than predicted. Overall the results agree quite well with theory demonstrating the ability of the TDMA in measuring aerosol charge distributions.

Table C1. Instrument settings and parameters used for TDMA and SMPS measurements

\begin{tabular}{lcc}
\hline & DMA-1 & SMPS (DMA-2 + CPC) \\
\hline Sheath flow rate $(1 / \mathrm{min})$ & 3.0 & 3.0 \\
Sample flow rate $(1 / \mathrm{min})$ & 0.3 & 0.3 \\
Aerosol flow rate $(1 / \mathrm{min})$ & 0.3 & 0.3 \\
DMA polarity & negative, positive & negative \\
Impactor $(\mathrm{cm})$ & no & 0.0457 \\
Impactor D50 $(\mathrm{nm})$ & & 439 \\
Charge neutralizer & no & TSI 3077A \\
Sample scan time $(\mathrm{sec})$ & & 270 \\
Time between samples $(\mathrm{sec})$ & & 200 \\
DMA voltage range $(\mathrm{V})$ & $20.4-6726$ & $10-9691$ \\
Measurable size range $(\mathrm{nm})$ & & $13.8-750$ \\
\hline \hline
\end{tabular}




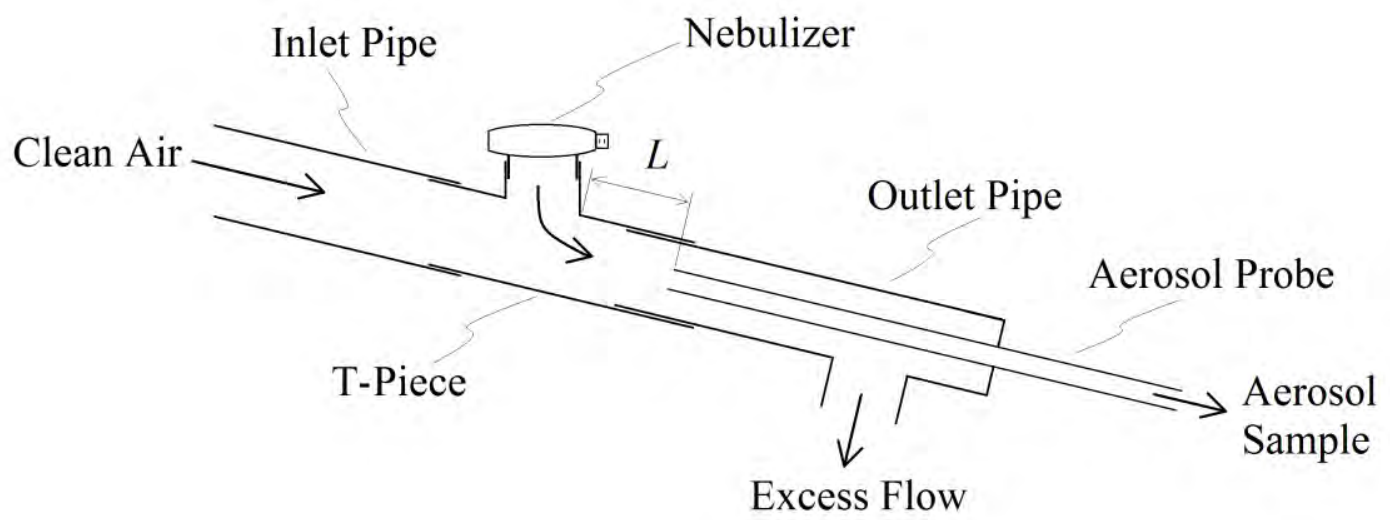

Figure $\mathrm{C} 1$. Aerosol generation apparatus including pharmaceutical nebulizer, air duct, and aerosol sampling probe $(L=200 \mathrm{~mm})$.
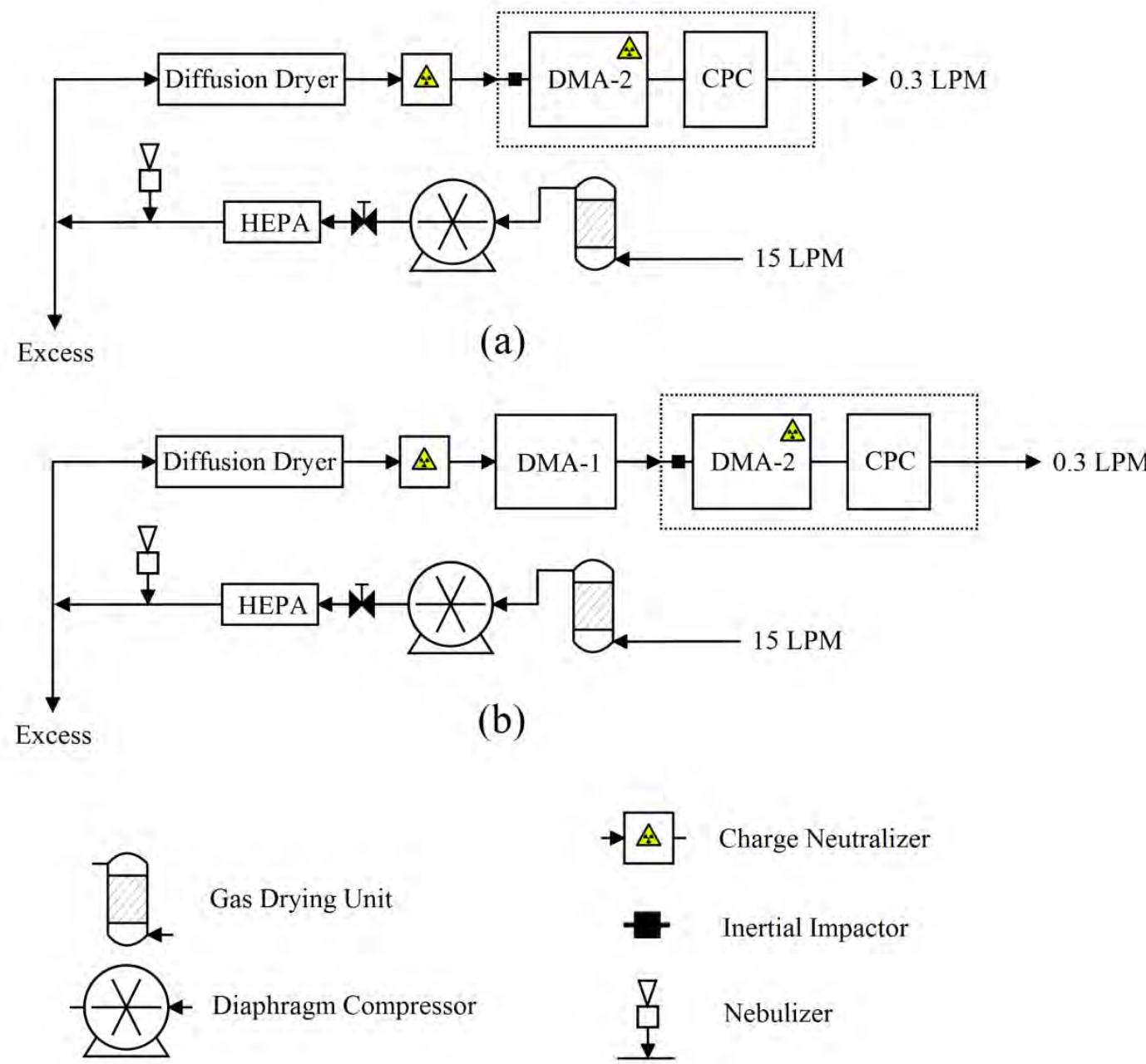

Figure C2. Schematic diagram of (a) SMPS measurements for charged neutralized aerosol (b) TDMA measurements for charge neutralized aerosol. 


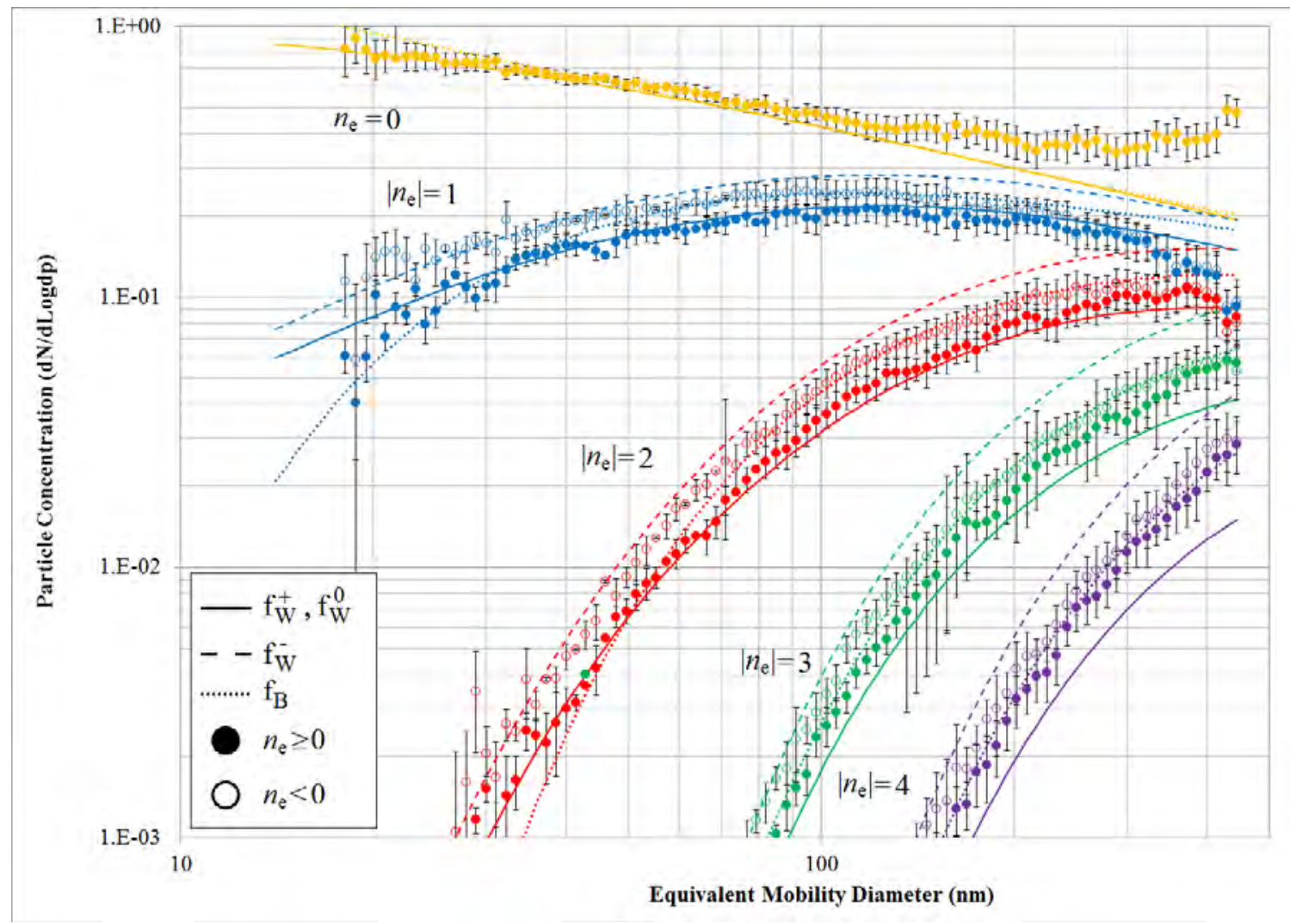

Figure $\mathrm{C} 3$. Measured charge fractions for charge neutralized $\mathrm{NaCl}$ aerosol with comparisons against the corrected Fuchs $\left(\mathrm{f}_{\mathrm{W}}\right)$ and Boltzmann $\left(\mathrm{f}_{\mathrm{B}}\right)$ charge distributions (Flagan 2001). 


\section{Appendix D \\ SAMPLING PROBE CAP AND PORT CAP DESIGN}

The sampling ports along the length of the coagulation chamber need to be sealed when not in use to prevent disturbances upstream of the aerosol sampling location. This was accomplished using plastic inserts made from ABS thermoplastic using a fused deposition modeling (FDM) machine available in the rapid prototyping lab at the University of Missouri College of Engineering. The caps are designed with an O-ring seal and a recessed groove to locate a band clamp around the outside. Engineering drawings of the port cap and probe cap are shown below in Figures D1 \& D2, respectively. 


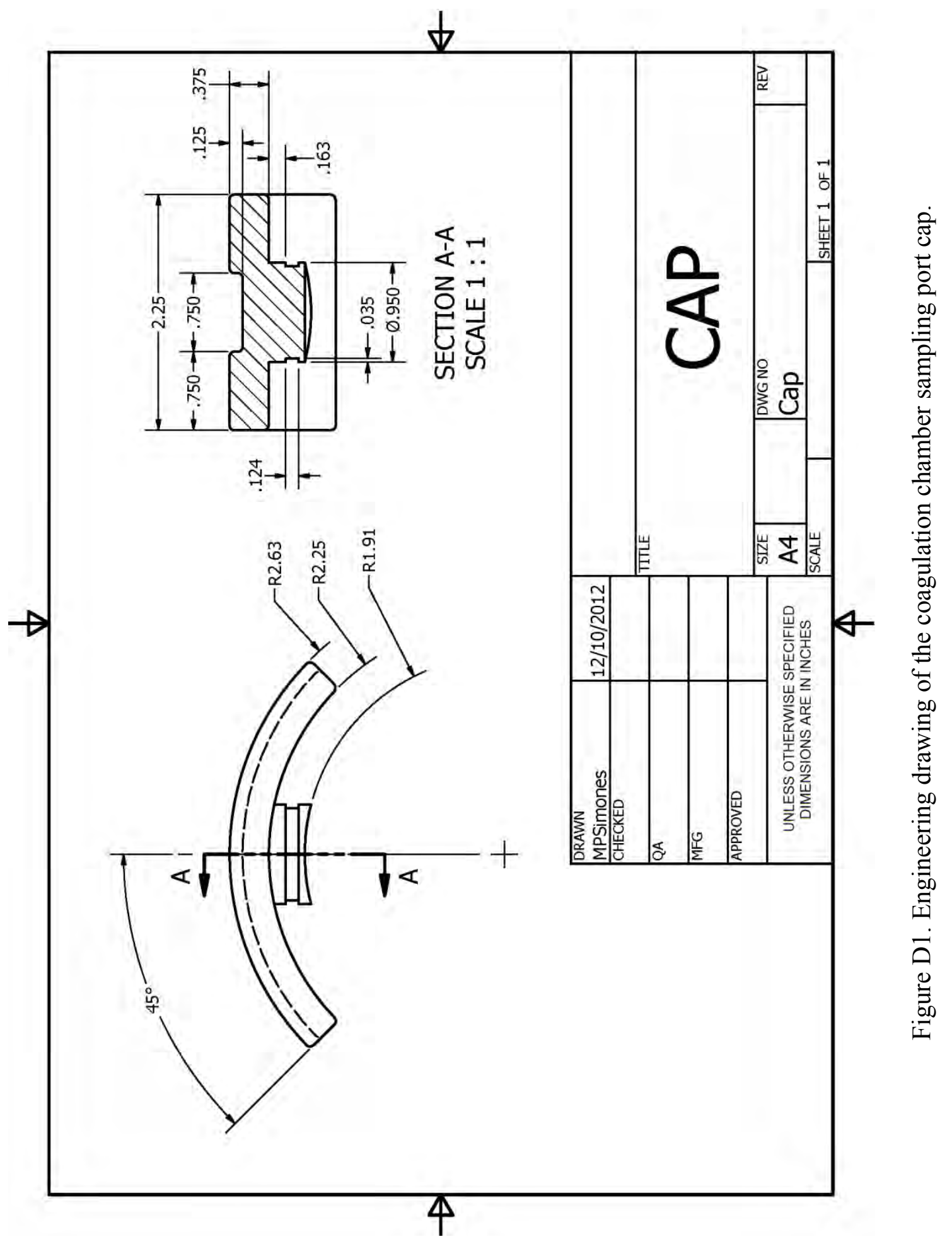




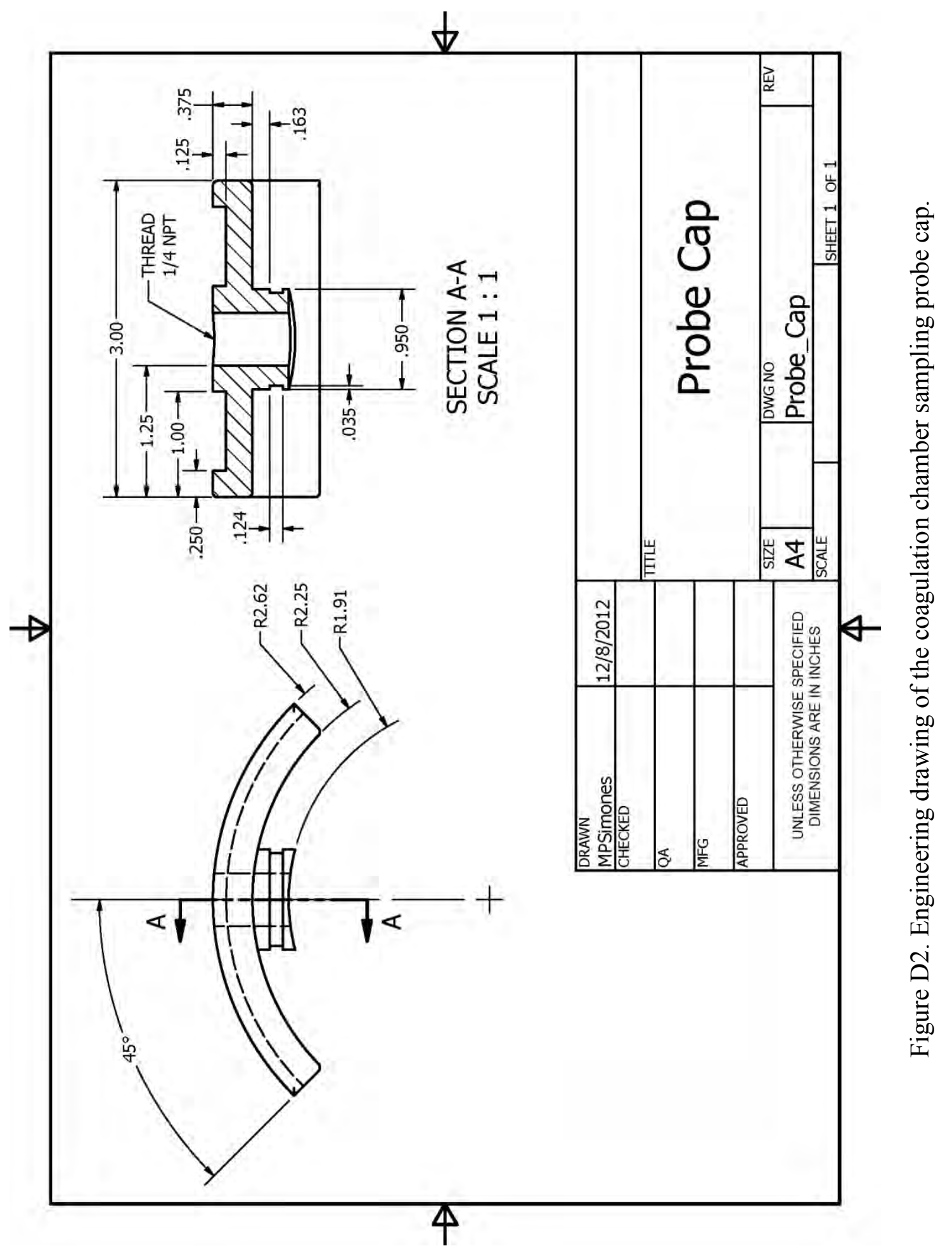




\section{Appendix E \\ DIFFUSER AND NOZZLE DESIGN}

The inlet diffuser to the coagulation chamber was designed to expand the supplied aerosol from the transport tubing diameter $(8 \mathrm{~mm})$ to the coagulation chamber diameter $(97 \mathrm{~mm})$ at a flow rate $1.5 \mathrm{~L} / \mathrm{min}$ while both minimizing flow recirculation (boundary layer separation) and overall diffuser length. Since the expansion of a fluid is inherently unstable, diffusers are generally restricted to expansion angles less than $15^{\circ}$. In the current situation with a large change in diameter this restriction leads to a very long diffuser which then restricts the coagulation test section. To shorten the overall length, conical deflectors were introduced as suggested by Cheng (1992) which essentially divides the diffuser into smaller regions. Cheng recommends arranging the deflectors such that the inner cone has an expansion angle of $15^{\circ}$ or less, successive outer cones are staggered in the flow direction along the central axis, the half-angle difference in expansion angle between any two adjacent cones is $7.5^{\circ}$ or less, and that each cone shares the same focal point on the central axis as that of the outer wall. For the coagulation chamber an overall expansion angle of $45^{\circ}$ was chosen and two deflectors were incorporated to divide the diffuser into three $7.5^{\circ}$ half-angle expansion regions. The staggered placement between adjacent deflectors was set so that the larger diameter deflector began at approximately the midpoint of the small deflector. The nozzle design was identical to the diffuser except for the omission of the internal deflectors. Figure E1 shows the diffuser flow simulation performed in COMSOL and Figures E2 \& E3 show the engineering drawings for the diffuser and nozzle, respectively. Both the nozzle and 
diffuser were made from ABS thermoplastic using a fused deposition modeling (FDM) machine available in the rapid prototyping lab at the University of Missouri College of Engineering. During initial installation and testing it was found that both the nozzle and diffuser where slightly porous due to the limitations of the FDM technique. To make each piece gas tight they were coated in cyanoacrylate which was readily absorbed into the open pores. Additional features include two O-ring glands to provide a pressure seal against the coagulation chamber and a tapered pipe thread (1/4 NPT) for connecting the aerosol transport tubing. 


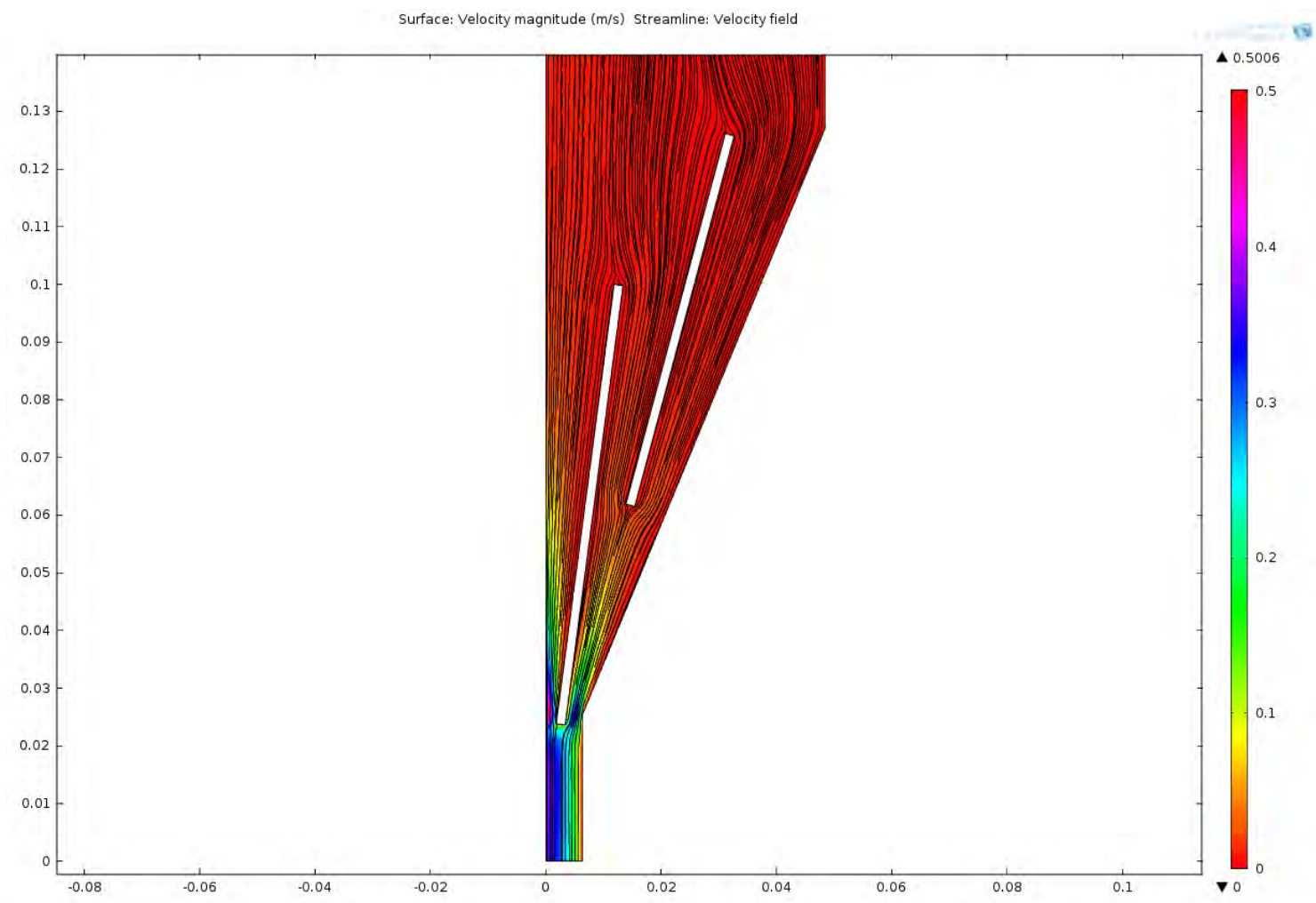

Figure E1. COMSOL axisymmetric flow model results of diffuser design with deflectors. Streamlines indicate smooth expansion without flow recirculation. Color legend is velocity magnitude ranging from $0-0.5 \mathrm{~m} / \mathrm{s}$, axial and radial coordinates given in meters. 


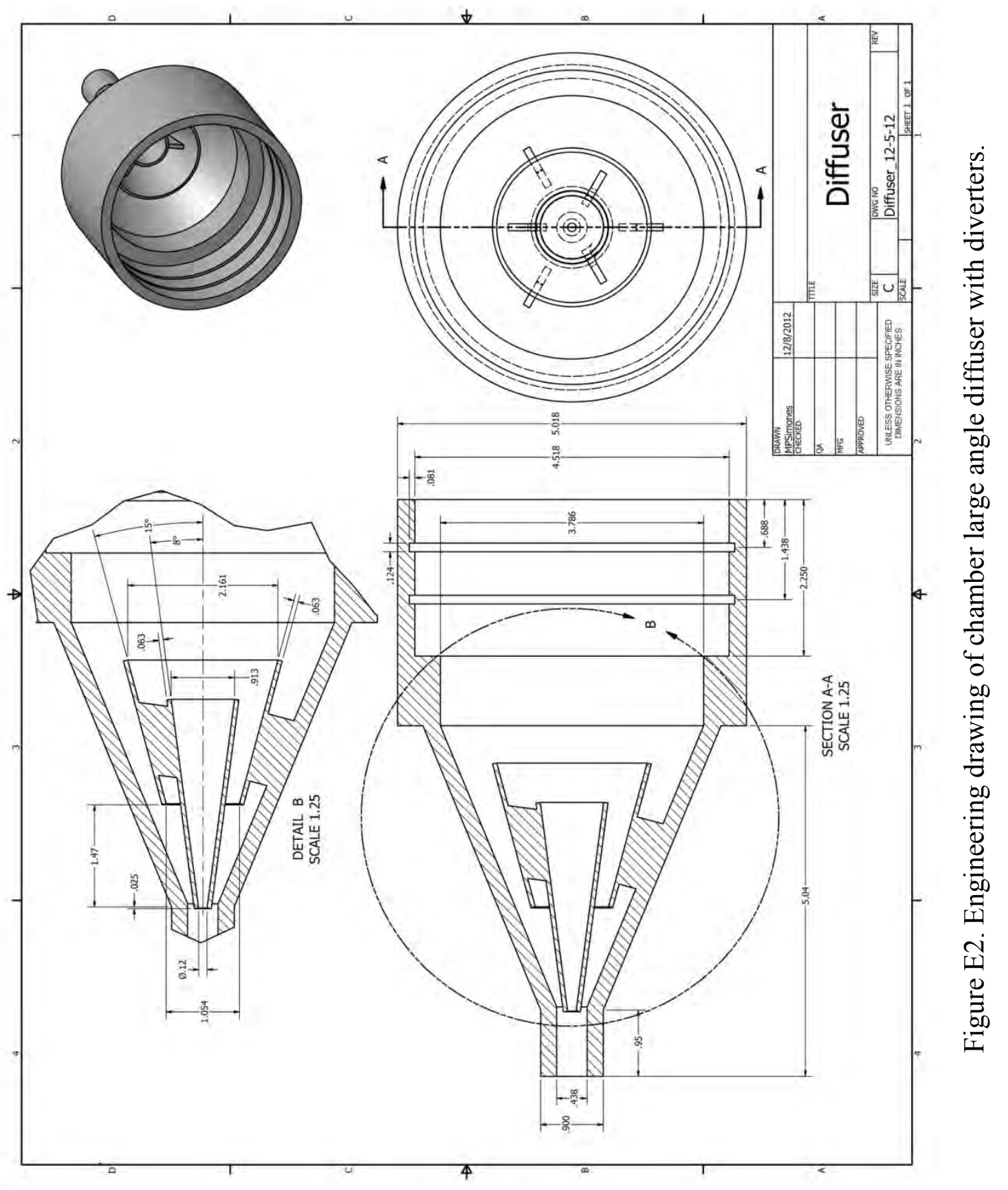




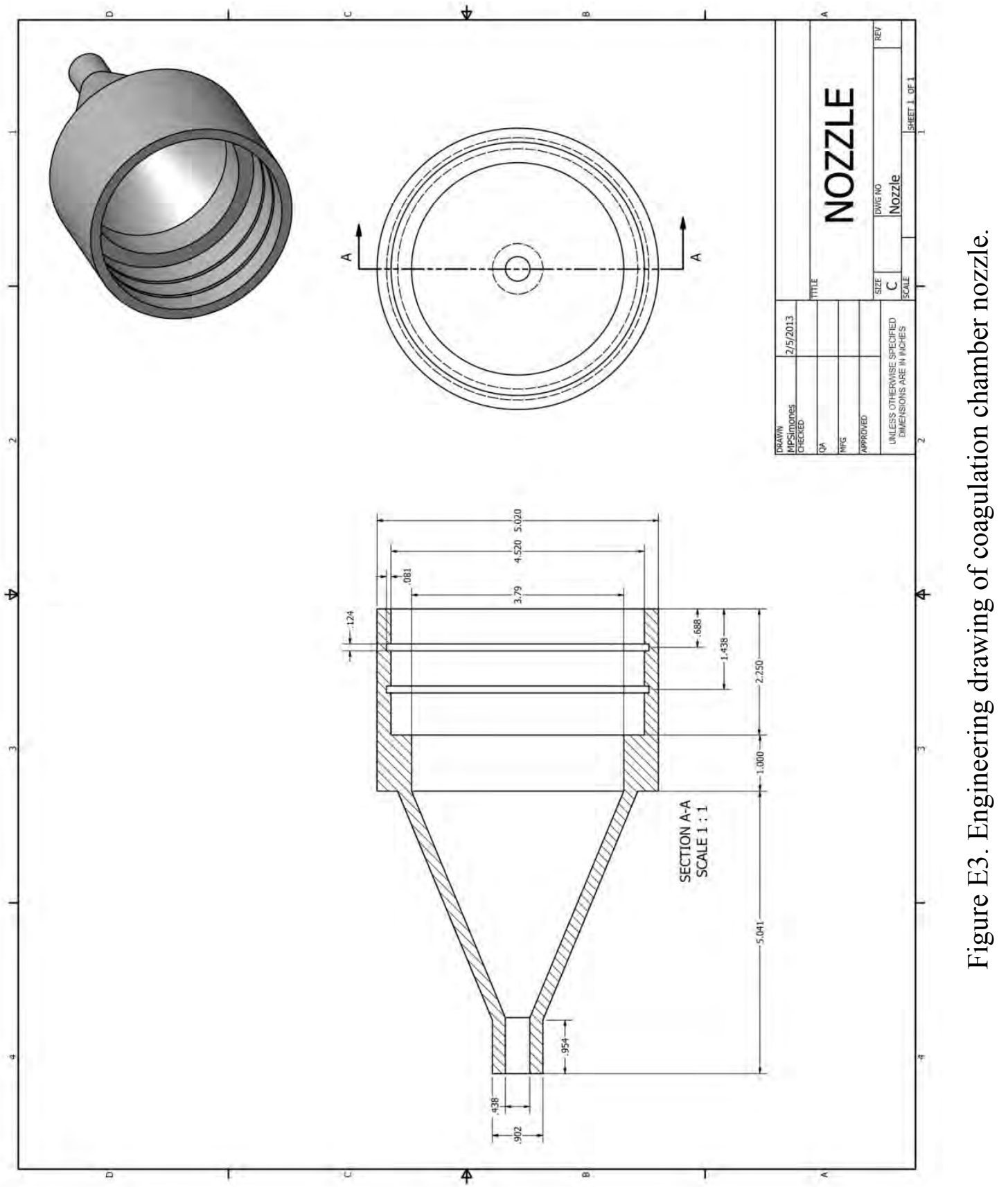




\section{Appendix F}

\section{COAGULATION CHAMBER DESIGN}

\section{Coagualtion Chamber Design}

\section{Parameters}

Fluid Properties \& Constants

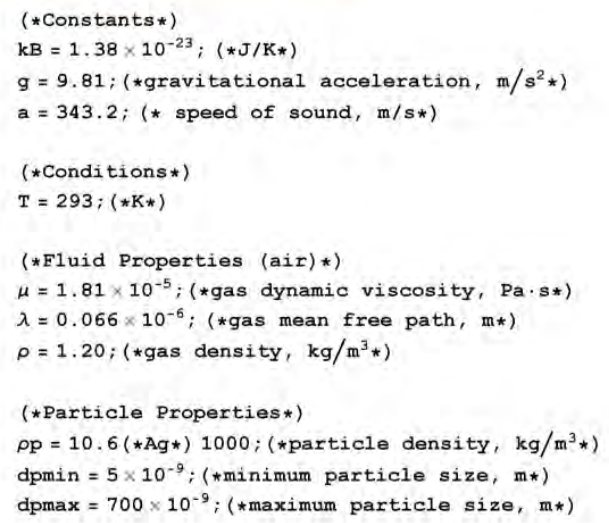

Aerosol Parameters \& Dimensionless Numbers

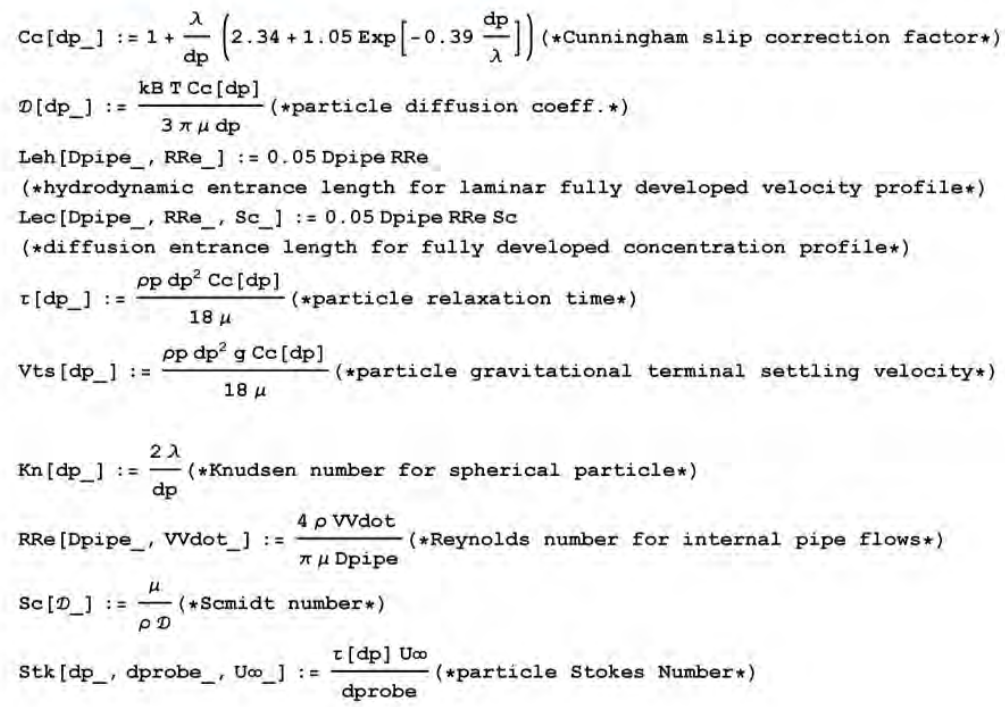


Pipe \& Tubing Size Selection

(* Pipe Size Chart *)

TableForm $[\{\{4,4.5,4.5-2 * 0.242,4.5-2 * 0.344,0.242,0.344\}$,

$\{5,5.563,5.563-2 * 0.263,5.563-2 * 0.383,0.263,0.383\}$,

$\{6,6.625,6.625-2 * 0.286,6.625-2 * 0.441,0.286,0.441\}$,

$\{8,8.625,8.625-2 * 0.329,8.625-2 * 0.510,0.329,0.510\}\}$, TableHeadings $\rightarrow\{\{\}$,

\{"Nominal", "OD", "ID, Sch 40", "ID, Sch 80", "Wall, Sch 40", "Wall, Sch 80"\}\}]

\begin{tabular}{lllllll} 
Nominal & OD & ID, Sch 40 & ID, Sch 80 & WaIl, Sch 40 & Wall, Sch 80 \\
\hline 4 & 4.5 & 4.016 & 3.812 & 0.242 & 0.344 \\
5 & 5.563 & 5.037 & 4.797 & 0.263 & 0.383 \\
6 & 6.625 & 6.053 & 5.743 & 0.286 & 0.441 \\
8 & 8.625 & 7.967 & 7.605 & 0.329 & 0.51
\end{tabular}

dtube $=0.31(0.0254) ;(*$ tube diameter (from spark gen.), in $\rightarrow$ m )

dpipe $=3.812(0.0254)$;

(* pipe inside diameter (coagulation chamber size), in $\rightarrow \mathrm{m} *$ )

dinlet $=$ dtube $;(\star$ diffuser inlet diameter, in $\rightarrow m *$ )

RRecrit $=2100$;

$I=110(0.0254) ;(*$ pipe length, in $\rightarrow m *)$

vdot $=1.5\left(\frac{10^{-3}}{1}\right)\left(\frac{1}{60}\right) ;\left(*\right.$ volumetric flowrate, $\left.1 / \mathrm{min} \rightarrow \mathrm{m}^{3} / \mathrm{sec} *\right)$

Print ["Mean velocity in tubing $\left.=", \frac{\text { Vdot }}{\pi(\text { dtube } / 2)^{\star} 2}, " \mathrm{~m} / \mathrm{s} ", " \backslash \mathrm{n} "\right]$

Print ["Mean velocity in pipe $\left.=", \frac{\text { vdot }}{\pi(\text { dpipe /2)^ } 2}, " \mathrm{~m} / \mathrm{s} ", " \backslash \mathrm{n} "\right]$

Mean velocity in tubing $=0.513404 \mathrm{~m} / \mathrm{s}$

Mean velocity in pipe $=0.00339529 \mathrm{~m} / \mathrm{s}$

Determine maximum flow rate which preserves laminar flow through small tubing

Print ["Qcrit $=$ ",

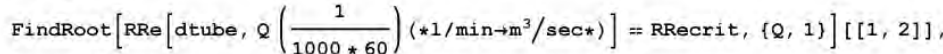

" $1 / \mathrm{min} "]$

Qcrit $=11.7531 \mathrm{l} / \mathrm{min}$

\section{Simple Monodisperse Coagulation}

(*Fuchs corrected monodisperse coagulation coefficient, Hinds Table A11*) $\mathrm{KK}=$ Interpolation $\left[\left\{\left\{1 \times 10^{-9}, 3.11 \times 10^{-16}\right\},\left\{1.5 \times 10^{-9}, 3.81 \times 10^{-16}\right\}\right.\right.$,

$\left\{2 \times 10^{-9}, 4.40 \times 10^{-16}\right\},\left\{3 \times 10^{-9}, 5.39 \times 10^{-16}\right\},\left\{4 \times 10^{-9}, 6.21 \times 10^{-16}\right\}$,

$\left\{5 \times 10^{-9}, 6.93 \times 10^{-16}\right\},\left\{6 \times 10^{-9}, 7.56 \times 10^{-16}\right\},\left\{8 \times 10^{-9}, 8.63 \times 10^{-16}\right\}$,

$\left\{10 \times 10^{-9}, 9.48 \times 10^{-16}\right\},\left\{15 \times 10^{-9}, 1.09 \times 10^{-15}\right\},\left\{20 \times 10^{-9}, 1.15 \times 10^{-15}\right\}$,

$\left\{30 \times 10^{-9}, 1.14 \times 10^{-15}\right\},\left\{40 \times 10^{-9}, 1.07 \times 10^{-15}\right\},\left\{50 \times 10^{-9}, 9.92 \times 10^{-16}\right\}$,

$\left\{60 \times 10^{-9}, 9.20 \times 10^{-16}\right\},\left\{80 \times 10^{-9}, 8.03 \times 10^{-16}\right\},\left\{100 \times 10^{-9}, 7.17 \times 10^{-16}\right\}$,

$\left.\left.\left\{150 \times 10^{-9}, 5.83 \times 10^{-16}\right\},\left\{200 \times 10^{-9}, 5.09 \times 10^{-16}\right\},\left\{300 \times 10^{-9}, 4.34 \times 10^{-16}\right\}\right\}\right]$;

$\mathrm{P} 10 t\left[\mathrm{KK}[\mathrm{dp}],\left\{\mathrm{dp}, 5 \times 10^{-9}, 300 \times 10^{-9}\right\}\right]$

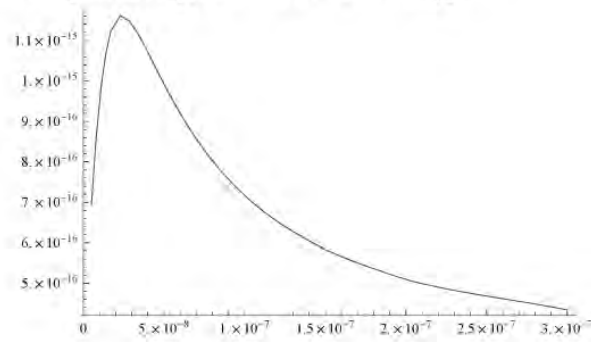



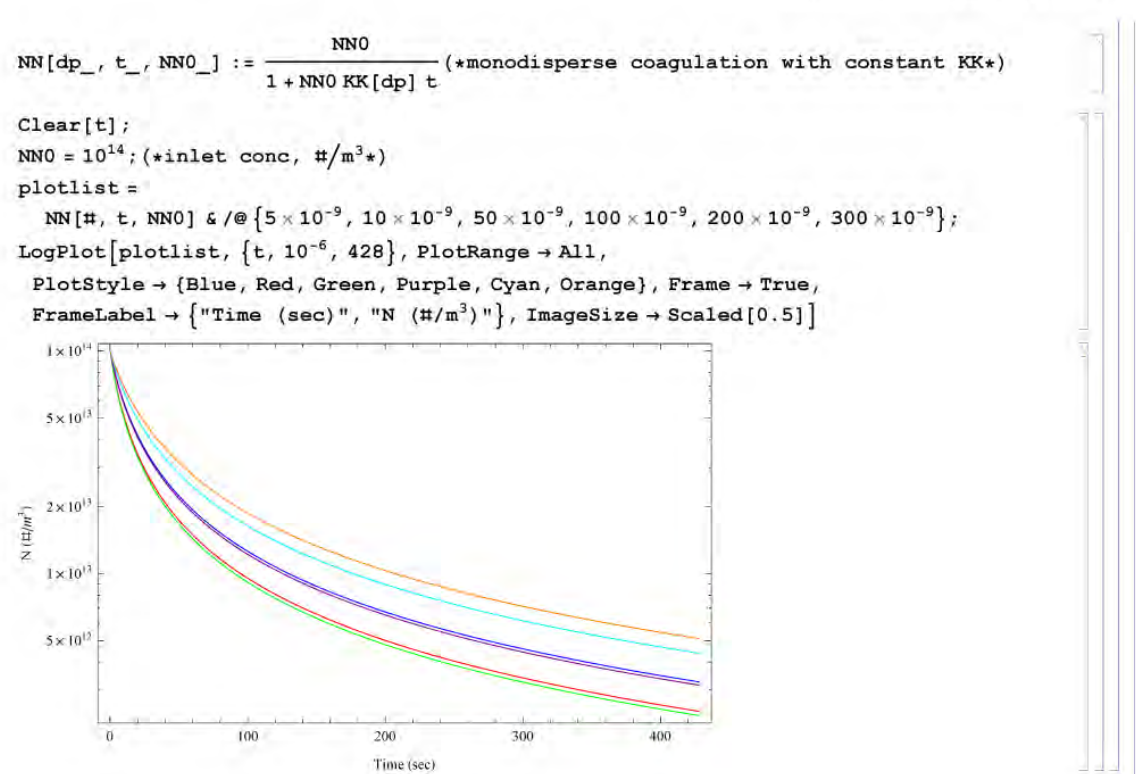

\section{Simple monodisperse diffusional deposition}

$\mu$ dep $\left[I_{-}, D_{-}\right.$, Vdot_ $]:=\pi \mathrm{DD} L_{1} /$ Vdot

$\mathrm{P}[\mu \mathrm{dep}]=1.0-2.5638 \mu \mathrm{dep}^{\frac{2}{3}}+1.2 \mu \mathrm{dep}+0.1767 \mu \mathrm{dep}^{4 / 3} / ; \mu \mathrm{dep} \leq 0.02$

$\mathrm{P}[\mu \mathrm{dep}]:=0.81905 \mathrm{Exp}[-3.6568 \mu \mathrm{dep}]+0.09753 \mathrm{Exp}[-22.305 \mu \mathrm{dep}]+$

$0.0325 \operatorname{Exp}[-56.961 \mu$ dep $]+0.01544 \operatorname{Exp}[-107.62 \mu$ dep $] / ; \mu$ dep $>0.02$

plot 1 ist $=\mathrm{P}[\mu \operatorname{dep}[\mathrm{LL}, \mathcal{D}[\#], \operatorname{Vdot}]] \& / @\left\{5 \times 10^{-9}, 10^{-8}, 10^{-7}\right\}$;

Plot [plotlist, $\{$ LL, 0,2$\}$, PlotPoints $\rightarrow 100$, MaxRecursion $\rightarrow 10$,

PlotStyle $\rightarrow$ \{Red, Blue, Orange $\},$ PlotLegends $\rightarrow$ SwatchLegend ["Expressions"]]

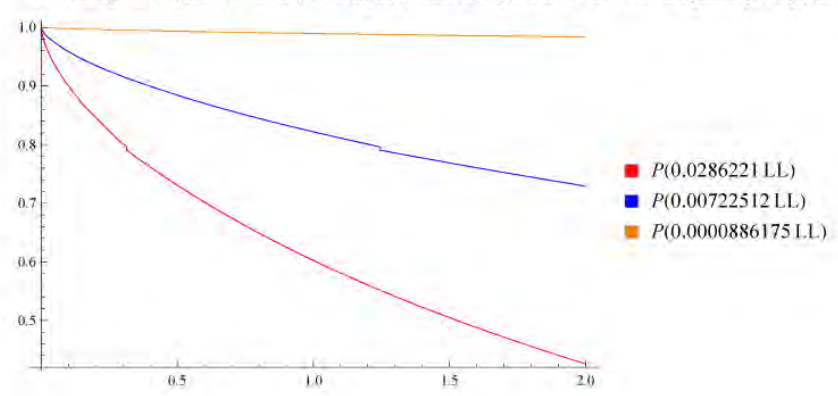




\section{Chamber Geometry/Parameters}

$v z\left[r_{-}\right]:=\frac{2 \text { Vdot }}{\pi(\text { dpipe } / 2)^{2}}\left(1-\left(\frac{2 r}{\text { dpipe }}\right)^{2}\right)(*$ velocity profile, Poiseuille flow $*)$

vzmax $=\mathrm{vz}[0] ;(* \operatorname{maximum}$ velocity*)

vzbar $=\mathrm{vz}[0] / 2 ;(*$ average velocity $*)$

$\mathrm{Ma}=\mathrm{vzmax} / \mathrm{a} ;(*$ Mach number $*)$

Print [" $\backslash \mathrm{n} \backslash \mathrm{n} "]$

Plot [vz [r], $\{r,-$ dpipe / 2, dpipe / 2\}, AspectRatio $\rightarrow .1$, ImageSize $\rightarrow$ Large $]$

Print ["\n", "Volumetric flow rate $\left.(1 / \mathrm{min})=", \operatorname{vdot}\left(60 / 10^{-3}\right)\right]$

Print ["Pipe inside diameter $($ in) $="$, dpipe $/(0.0254)]$

Print ["Fluid velocity at center $(\max )="$, vzmax, $" \mathrm{~m} / \mathrm{s} "]$

Print ["Average fluid velocity = ", vzbar, " $\mathrm{m} / \mathrm{s} "]$

Print ["residence time of fluid at center (vz=vzmax) $=", \mathrm{~L} / \mathrm{vzmax}, " \mathrm{sec}$ "]

Print ["mean residence time of fluid (vz=vzbar) = ", L/vzbar, " sec"]

Print ["Reynolds \# at diffuser entrance $=", \operatorname{RRe}[$ dtube, Vdot] ]

Print ["Reynolds \# in pipe $=", \operatorname{RRe}[$ dpipe, Vdot] ]

Print ["Entrance length at diffusser inlet = ", Leh [dinlet, Vdot], " m"]

Print ["Entrance length at pipe inlet (uniform flow at entrance) = ",

Leh [dpipe, RRe [dpipe, vdot]], " m"]

Print["Diffusional Entrance length at pipe inlet (uniform flow at entrance, $\mathrm{dp}=\operatorname{dpmin})=", \operatorname{Lec}[$ dpipe, $\operatorname{RRe}[$ dpipe, $\operatorname{Vdot}], \operatorname{Sc}[\mathcal{D}[$ dpmin] $]], " \mathrm{~m} "]$

Print["Diffusional Entrance length at pipe inlet (uniform flow at entrance,

$\mathrm{dp}=$ dpmax $)=", \operatorname{Lec}[$ dpipe, $\operatorname{RRe}[$ dpipe, $\operatorname{vdot}], \mathrm{Sc}[\mathcal{D}[$ dpmax] $]], " \mathrm{~m} "]$

Print ["vzmax $="$, vzmax, $" \backslash \mathrm{n} "$, "vzbar $="$, vzbar, $" \backslash \mathrm{n} ", " \mathrm{Ma}=", \mathrm{Ma}]$

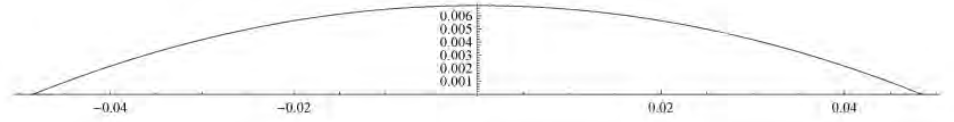

Volumetric flow rate $(\mathrm{I} / \mathrm{min})=1.5$

Pipe inside diameter $($ in $)=3.812$

Fluid velocity at center $(\max )=0.00679058 \mathrm{~m} / \mathrm{s}$

Average fluid velocity $=0.00339529 \mathrm{~m} / \mathrm{s}$

residence time of fluid at center $(v z=v z \max )=411,452 \mathrm{sec}$

mean residence time of fluid ( $v z=v z$ bar $)=822.905 \mathrm{sec}$

Reynolds \# at diffuser entrance $=261.871$

Reynolds $\#$ in pipe $=21,2959$

Entrance length at diffusser inlet $=9.8425 \times 10^{-9} \mathrm{~m}$

Entrance length at pipe inlet (uniform flow at entrance) $=0.103098 \mathrm{~m}$

Diffusional Entrance length at pipe inlet (uniform flow at entrance, dp=dpmin) $=6.9876 \mathrm{~m}$

Diffusional Entrance length at pipe inlet (uniform flow at entrance, $d p=d p m a x$ ) = $38508.8 \mathrm{~m}$

vzmax $=0.00679058$

vzbar $=0.00339529$

$\mathrm{Ma}=0.0000197861$ 
Diffuser

$\alpha=22.5 * \mathrm{Pi} / 180$;

Plot $\left[\right.$ FindRoot $\left[\operatorname{Tan}[\alpha]=\frac{(\text { dd } * 0.0254-\text { dinlet }) / 2}{x},\{x, 1\}\right][[1,2]] / 0.0254$,

$\{d d$, dinlet $/ 0.0254,10\}$, Frame $\rightarrow$ True, Axes $\rightarrow$ False,

FrameLabel $\rightarrow\{$ "Pipe diameter (in)", "Diffuser length (in)" $\}]$

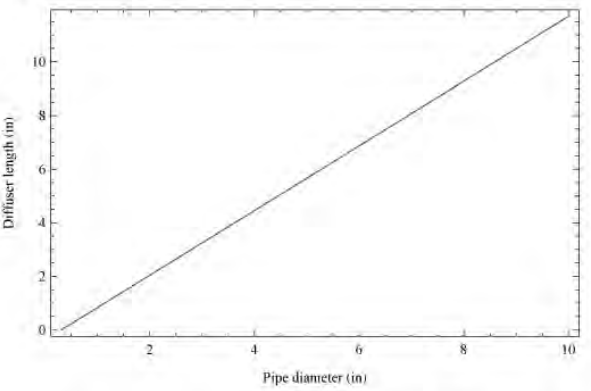

$\left.\operatorname{Ldiff}=\operatorname{FindRoot}\left[\operatorname{Tan}[\alpha]=\frac{(\text { dpipe }- \text { dinlet }) / 2}{x},\{x, 1\}\right][1,2]\right]$;

Print ["diffuser length (dpipe $="$,

dpipe / 0.0254, " in) = ", Ldiff / 0.0254, " in", "\n"]

Plot $[(\operatorname{Tan}[\alpha] * x * 0.0254+\operatorname{dinlet} / 2) / 0.0254,\{x, 0$, Ldiff $/ 0.0254\}$,

PlotRange $\rightarrow$ Full, Frame $\rightarrow$ True, PlotLabel $\rightarrow$ "Diffuser profile",

AspectRatio $\rightarrow 0.5$ dpipe / (Ldiff), ImageSize $\rightarrow$ Scaled [0.8]]

diffuser length (dpipe $=3.812 \mathrm{in})=4.22729 \mathrm{in}$

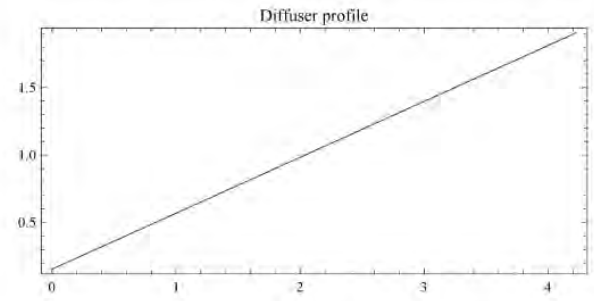

pointslist $=\{\#,(\operatorname{Tan}[\alpha] * \# * 0.0254+\operatorname{dinlet} / 2) / 0.0254\} \& / @$ Append [Range [0, Ldiff / 0.0254,0.1], Ldiff / 0.0254] ;

Nozzle

$\theta=22.5 * \mathrm{Pi} / 180$

Plot $\left[\right.$ FindRoot $\left[\operatorname{Tan}[\theta]=\frac{(d d * 0.0254-\text { dinlet }) / 2}{x},\{x, 1\}\right][[1,2]] / 0.0254$, $\{$ dd, dinlet $/ 0.0254,10\}$, Frame $\rightarrow$ True, Axes $\rightarrow$ False,

FrameLabel $\rightarrow\{$ "Pipe diameter (in)", "Nozzle length (in)" $\}]$

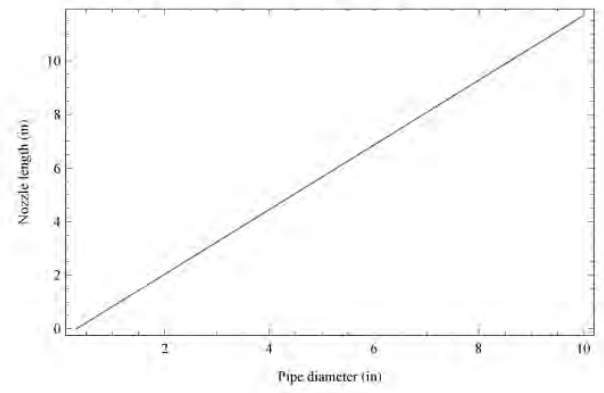


6 | Coagulation Chamber Design_1,1,1,nb
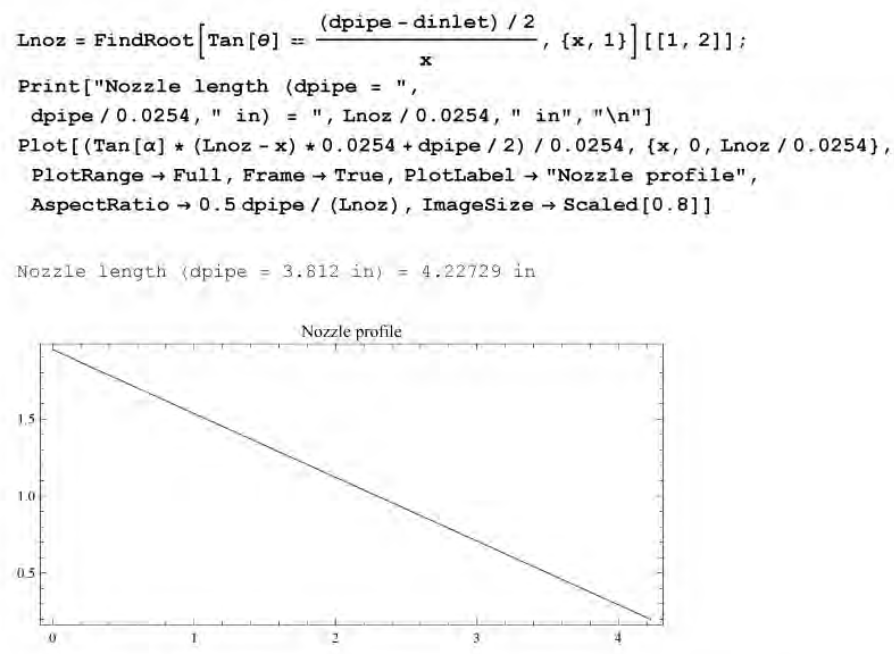

pointslistnoz $=$

$\{($ Ldiff $+L) / 0.0254+\#,(\operatorname{Tan}[\theta] *(\operatorname{Lnoz}-\#) * 0.0254+$ dpipe / 2) 10.0254$\} \& / @$ Append [Range $[0$, Lnoz / 0.0254,0.1], Lnoz / 0.0254] ;

\section{Chamber}

Print [" $\backslash n ",$, Total Length $(\mathrm{ft})=",(\mathrm{~L}+\operatorname{Ldiff}+\operatorname{Lnoz}) * 3.28]$

Print ["Diffuser length $(\mathrm{ft})="$, Ldiff*3.28]

Print["Nozzle length $(\mathrm{ft})="$, Lnoz, "\n"]

Total Length $\langle\mathrm{ft}\rangle=9.86869$

Diffuser length $(\mathrm{ft}\rangle=0.352184$

Nozzle length $(\mathrm{ft})=0.107373$

ListPlot [Join [pointslist, pointslistnoz],

AspectRatio $\rightarrow 0.5$ dpipe $/(L+$ Ldiff + Lnoz $)$, Joined $\rightarrow$ True, ImageSize $\rightarrow$ Scaled [1] $]$

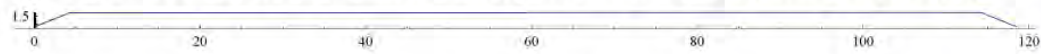

$f=$ Interpolation [Join [pointslist, pointslistnoz], Interpolationorder $\rightarrow 1$ ] ; RevolutionPlot3D $[f[x],\{x, 0,($ Ldiff $+\operatorname{Lnoz}+L) / 0.0254\}$, RevolutionAxis $\rightarrow\{1,0,0\}]$

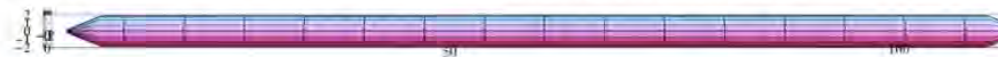

Sampling Probe

$v z \max * \frac{\pi}{4}\left(\right.$ dprobe $\left.^{2}\right) * 1000 * 60$

0.00098291

Stk [dpmax, dprobe, vzmax]

Stk [dpmax, dprobe, Usam]

0.0000754955

0.0115212 
dprobe $=.069(0.0254) ;(*$ Inside diameter of sampling probe, in $\rightarrow m *)$

Vdotsampling $=1.5 ;(*$ CPC sampling flow rate, $\mathrm{L} / \mathrm{min} *)$

Vdotprobe $=0.15 /(1000 * 60) ;\left(*\right.$ Usam $*\left(\frac{\pi}{4}\right)$ dprobe $^{2}(1000 * 60)$;

(*volumetric flow rate in sampling probe, $\mathrm{m}^{3} / \mathrm{s} \rightarrow \mathrm{L} / \mathrm{min} *$ ) *)

Usam $=$ Vdotprobe $4 /\left(\pi\right.$ dprobe $\left.^{2}\right)$;

(*Integrate $[\mathrm{vz}[r] \star r,(r, 0$, dprobe/2\}] / Integrate $[r,\{r, 0$, dprobe/2\}]; $)$

$(*$ mean velocity entering probe*)

Print ["Vdotprobe $="$, Vdotprobe, $\mathrm{L} / \mathrm{min} "]$

Print ["Vdotprobe/Vdotsampling (dilution) = ", Vdotprobe/Vdotsampling *100, " \%"]

Print ["Maximum sampling velocity variation across probe inlet $="$,

(vzmax - vz [dprobe / 2]) / vzmax * 100, "q"]

Print ["Calm air velocity criterion (dpmax): Stk = ",

$t=0.1$ Usam / Stk [ dpmax, dprobe, vzmax $] \wedge 2 / 3, ">n$, vzmax,

Graphics [\{If[t>vzmax, Green, Red], Disk[]\}, ImageSize $\rightarrow$ Scaled[0.01]]]

Print ["Calm air velocity criterion (dpmin): Stk = ",

$t=0.1$ Usam / Stk [dpmin, dprobe, vzmax ]^ $2 / 3, ">n$, vzmax,

Graphics [\{If [ $t>\operatorname{vzmax}$, Green, Red], Disk[]\}, ImageSize $\rightarrow$ Scaled [0.01]]]

Print ["Calm air inertial condition (dpmax): stk $_{i}="$,

$t=S t k$ [dpmax, dprobe, Usam], "s 0.016 "

Graphics [(If [t $\leq 0.016$, Green, Red], Disk[]\}, ImageSize $\rightarrow$ Scaled [0.01]]]

Print ["Calm air inertial condition (dpmin): $\mathbf{s t k}_{i}="$

$t=S t k$ [dpmin, dprobe, Usam], "s $0.016 "$

Graphics [(If [ $t \leq 0.016$, Green, Red], Disk[]), ImageSize $\rightarrow$ Scaled [0.01]]]

Print ["Calm air settling velocity condition (dpmax): $\frac{\mathrm{v}_{\mathrm{ts}}}{\mathrm{U}_{\mathrm{sam}}}="$,

$t=V t s[$ dpmax ] / Usam, " $\leq 0.04 "$

Graphics [\{If[t $\leq 0.04$, Green, Red], Disk[]\}, ImageSize $\rightarrow$ Scaled [0.01]]]

Print ["Calm air settling velocity condition (dpmin): $\frac{v_{t s}}{U_{s}}=$ ",

$\mathrm{t}=\mathrm{Vts}[\mathrm{dpmin}] /$ Usam, $" \leq 0.04 \mathrm{~N}$,

Graphics [ If [t $\leq 0.04$, Green, Red], Disk[]\}, ImageSize $\rightarrow$ Scaled [0.01]]

Votprobe $=2.5 \times 10^{-6} \mathrm{~L} / \mathrm{min}$

Vdotprobe/Vdotsampling (dilution) $=0.000166667 \%$

Masimum sampling velocity variation across probe inlet $=0.0327637 \%$

CaIm air velocity criterion (dpmaz): Sth $=6.06068 \times 10^{6}>0.00679058$,

Calm air velocity criterion (dpmin): $s t k=1.69152 \times 10^{12}>0.00679058$ 。

Calm air inertial condition (dprax): $S t k_{t}=0.0115212 \leq 0.016$.

Calm ais inertial condition (dprin): Stk $=0,000021808350.016$.

Calm air settling velocity condition (dpmax): $\frac{V_{t s}}{U_{\sin }}=0.000184451 \leq 0.04$.

Calm air settling velocity condition (dpmin): $\frac{V_{t s}}{D_{\text {gad }}}=3.49143 \times 10^{-7} \leq 0.04$.

What is is the maximum sampling velocity that can be obtained before the calm air conditions are not met? Is this maximum velocity greater than the critical velocity for laminar flow?

Usamcrit $=$ FindRoot $[\rho \mathrm{v}$ dprobe $/ \mu=2000,\{\mathrm{v}, 1\}][[1,2]]$;

Usammax $=$ FindRoot $[$ Stk $[$ dpmax, dprobe, $v]=0.016,\{v, 1\}][[1,2]]$;

Print ["Critical sampling velocity $="$, Usamcrit]

Print ["Maximum sampling velocity = ", Usammax, " ",

Graphics [ $\{$ If [ $t<U$ sammax, Green, Red], Disk[] $\}, \operatorname{ImageSize} \rightarrow \operatorname{Scaled}[0.01]]]$

Critical sampling velocity $=17.2125$

Maximum sampling velocity $=1,43915$, 


\section{Appendix G}

\section{TDMA DATA ANALYSIS}

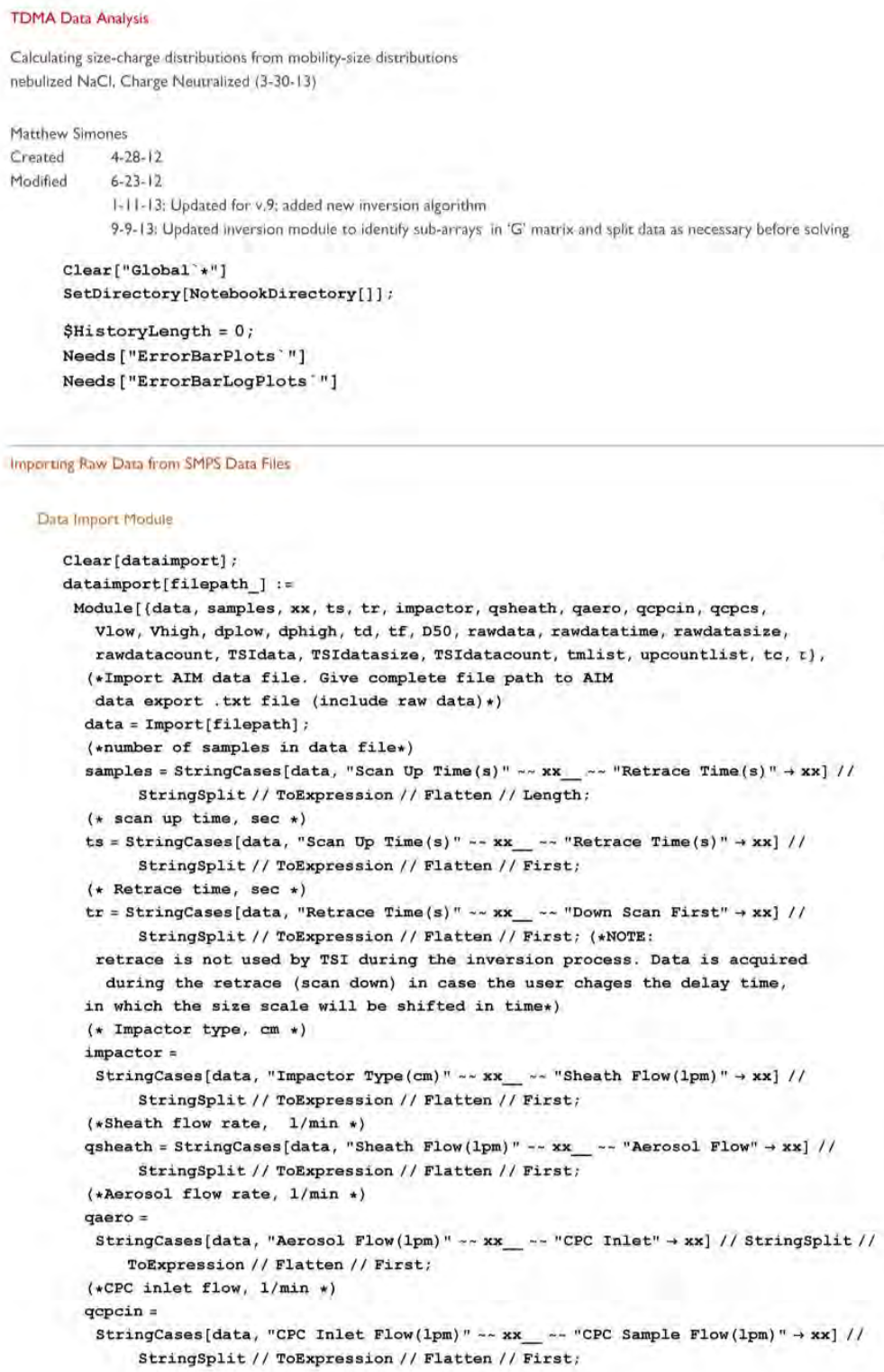




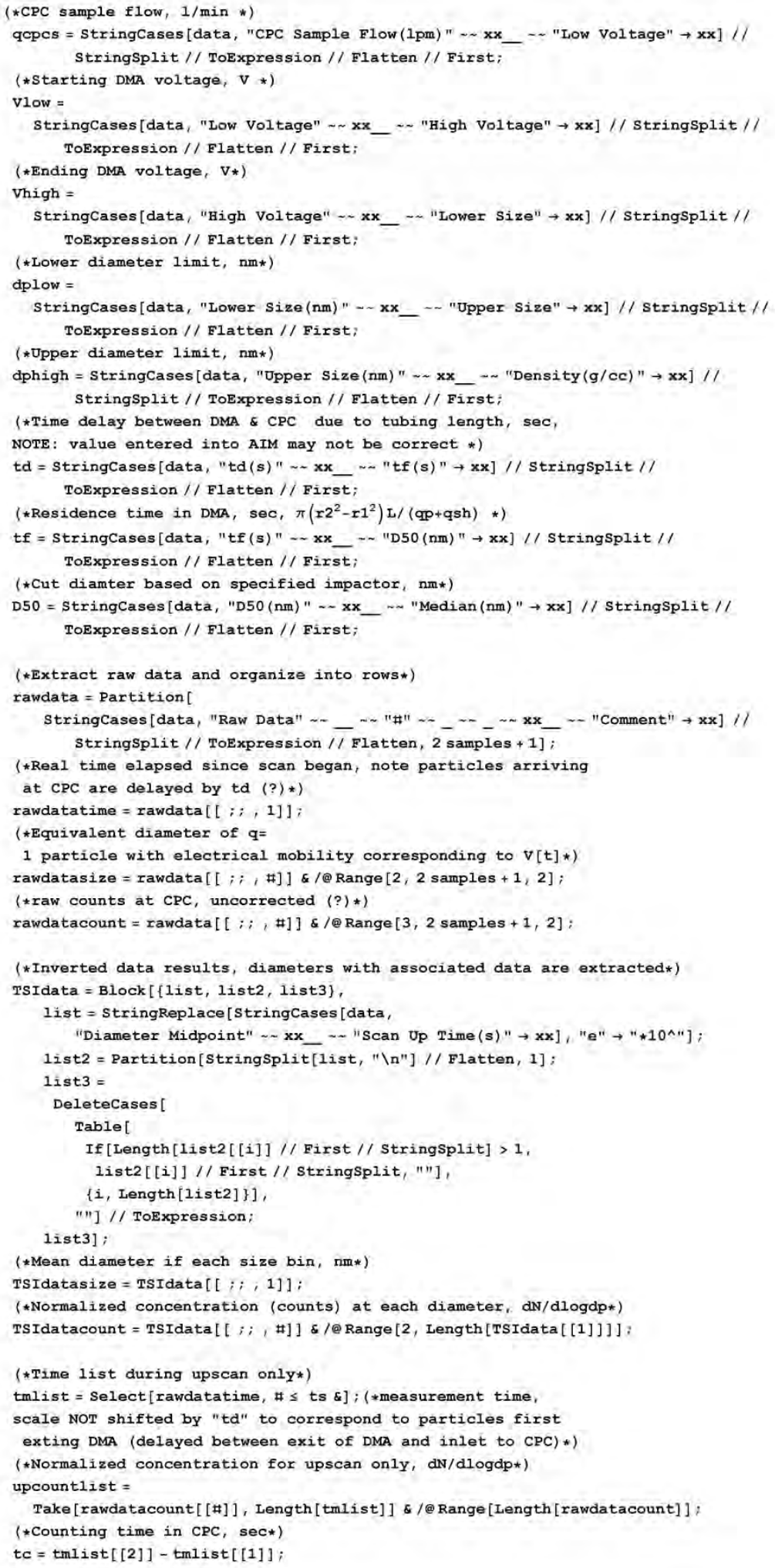


( $*$ DMA voltage time constant, sec*)

$\tau=\mathrm{ts} /$ Log $[$ Vhigh / Vlow $]$;

$\left\{\right.$ samples $(* 1 *), \operatorname{ts}(* 2 *), \operatorname{tr}(* 3 *)$, impactor $/ 100(* 4 *)$, qsheath $\left(10^{-3} / 60\right)$

$(* 5 *)$, qaero $\left(10^{-3} / 60\right)(* 6 *)$, qcpcin $\left(10^{-3} / 60\right)(* 7 *)$, qcpcs $\left(10^{-3} / 60\right)$

$(* 8 *)$, Vlow $(* 9 *)$, Vhigh $(* 10 *)$, dplow $(* 11 *)$, dphigh $(* 12 *)$, td

$(* 13 *)$, tf $(* 14 *), \mathrm{D} 50(* 15 *)$, rawdatatime $(* 16 *)$, rawdatasize $(* 17 *)$,

rawdatacount $(* 18 *)$, TSIdata $(* 19 *)$, TSIdatasize $(* 20 *)$, TSIdatacount

$(\star 21 *)$, tmlist $(\star 22 *)$, upcountlist $(\star 23 *)$, tc $(\star 24 *), \tau(* 25 *)\}]$

Import Raw Dita

DMA-1 voltages

(*Import example SMPS scan to determine

TDMA voltages based on bin diameters*)

\{samples, ts, tr, impactor, qsheath, qaero, qcpcin, qcpcs, Vlow, Vhigh, dplow, dphigh, td, tf, D50, rawdatatime, rawdatasize, rawdatacount, TSIdata, TSIdatasize, TSIdatacount, tmlist, upcountlist, tc, $\tau \tau\}=$ dataimport ["3-30-13_size range.txt"] ;

Total (SMPS)

( $*$ Import data \& parameters*)

(samplesSMPS, tsSMPS, trSMPS, impactorSMPS, qsheathSMPS, qaerosMPS, qcpcinSMPS, qcpcsSMPS, VlowSMPS, VhighsMPS, dplowSMPS, dphighsMPS, tdSMPS, tfSMPS, D50SMPS, rawdatatimeSMPS, rawdatasizeSMPS, rawdatacountSMPS, TSIdataSMPS, TSIdatasizeSMPS, TSIdatacountSMPS, tmlistSMPS, upcountlistSMPS, tcSMPS, $\tau$ SMPS $]=$ dataimport ["3-30-13_SMPS,txt"] ;

Positive Voltages (negative charges)

( $*$ Import data \& parameters $*$ )

(samplespos, tspos, trpos, impactorpos, qsheathDMApos, qaeropos, qcpcinpos, qcpcspos, vlowpos, Vhighpos, dplowpos, dphighpos, tdpos, tfpos, D50pos, rawdatatimepos, rawdatasizepos, rawdatacountpos, TSIdatapos, TSIdatasizepos, TSIdatacountpos, tmlistpos, upcountlistpos, tcpos, $\tau$ pos $]=$ dataimport ["3-30-13_TDMA_pos.txt"]

Negative Voltages (positive charges)

(*Import data \& parameters*)

(samplesneg, tsneg, trneg, impactorneg, qsheathDMAneg, qaeroneg,

qcpcinneg, qcpcsneg, vlowneg, Vhighneg, dplowneg, dphighneg, tdneg, tfneg, D50neg, rawdatatimeneg, rawdatasizeneg, rawdatacountneg,

TSIdataneg, TSIdatasizeneg, TSIdatacountneg, tmlistneg,

upcountlistneg, tcneg, $\tau$ neg $]=$ dataimport ["3-30-13_TDMA_neg.txt"] ;

Data Analysis

Parameters

qmax $=4 ;$ (*should be at least within the expected range from the equilibrium distribution*)

Physical Constants

$e=1.602 \times 10^{-19} ;$ ( $*$ fundamental unit of charge, $C *$ )

$\mathrm{kB}=1.38065 \times 10^{-23} ;(*$ Boltzmann Constant, $\mathrm{J} / \mathrm{K} *)$

gaccel $=9.81 ;\left(*\right.$ gravitational accel, $\left.\mathrm{m} / \mathrm{s}^{\wedge} 2 *\right)$

$\rho \mathrm{p}=2.16 ;(\star$ particle density $*)(\star \mathrm{NaCl} \star)$ 
Fluid Properties

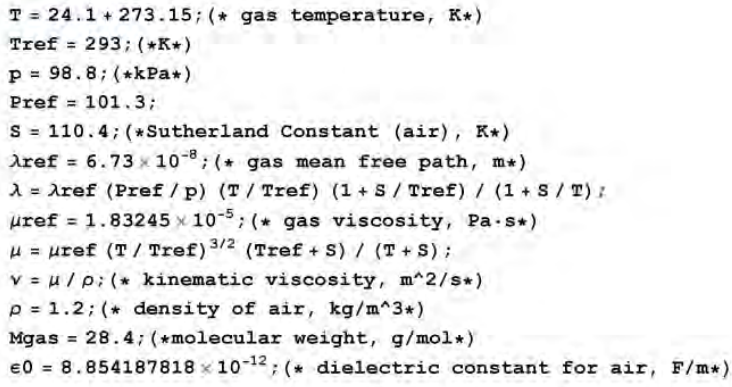

(* cunningham slip correction *)

$C c\left[d p_{-}\right]:=1+\frac{2 \lambda}{d p}\left(1,142+0,558 \operatorname{Exp}\left[-, 999 \frac{d p}{2 \lambda}\right]\right) ;$

(* Diffusion coefficient, $\mathrm{m}^{2} / \mathrm{s} *$ )

$\mathrm{DD}\left[\mathrm{dp}_{-}\right]:=\frac{\mathrm{kBTCC}[\mathrm{dp}]}{3 \pi \mu \mathrm{dp}}$;

(*particle relaxation time*)

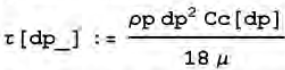

(*particle Stokes Number*)

Stk [dp_, dprobe_, U⿴_] $:=\frac{\tau[\mathrm{dp}] \mathrm{U \omega}}{\text { dprobe }}$

(*Penetration through circular tube with diffusive

deposition (Gormley \& Kennedy 1949), B\&W 2nd ed. p.580*)

$P\left[d_{-}, L_{-}, Q_{-}\right]:=$Module $[\{\xi$, penetration $\}$,

$\xi=\pi \frac{\mathrm{DD}[\mathrm{dp}] \mathrm{L}}{Q} ;$ (*penetration factor*)

penetration $:=1.0-2.5638 \xi^{2 / 3}+1.2 \xi+0.1767 \xi^{4 / 3} / ; \xi \leq 0.02$;

penetration $:=.81905 \operatorname{Exp}[-3.6568 \xi]+0.09753 \operatorname{Exp}[-22.305 \xi]+$

$0.0325 \operatorname{Exp}[-56.961 \xi]+0.01544 \operatorname{Exp}[-107.62 \xi] / ; \xi>0.02 ;$

penetration]; 
(*TSI 3077 charge neutralizer transmission data ( $Q=0.5$ LPM); Covert, Wiedensohler, Russel, 1997 *)

With $[\{\mathrm{L}=0.2$

(*estimated effective length (assumed to hold for $Q=0.3$ LPM) *) \}, Show $\left[\left\{\right.\right.$ ListPlot $\left[\left\{\left\{15 \times 10^{\wedge}-9,0.96\right\},\left\{10 \times 10^{\wedge}-9,0.94\right\}\right.\right.$,

$\left.\left.\left\{7 \times 10^{\wedge}-9,0.89\right\},\left\{5 \times 10^{\wedge}-9,0.83\right\},\left\{3 \times 10^{\wedge}-9,0.76\right\}\right\}\right]$,

Plot $\left[P[d p, L\right.$, qaeroSMPS $\left.\left.(0.5 / 0.3)],\left\{d p, 10^{\wedge}-9,20 \times 10^{\wedge}-9\right\}\right]\right\}$,

PlotRange $\rightarrow\left\{\left\{10^{-9}, 20 \times 10^{-9}\right\},\{0.5,1\}\right\}$, AxesOrigin $\left.\left.\rightarrow\left\{10^{-9}, 0.5\right\}\right]\right]$

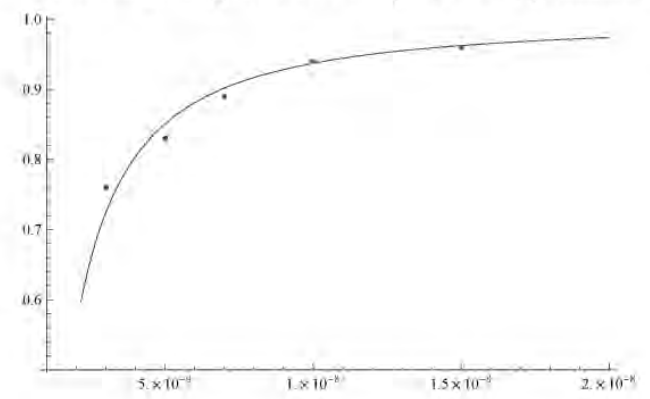

(*Penetration through a DMA, KarIsson \& Martinsson $2003 *$ ) PDMAKM [dp_, Qaero_] $:=$ Module $[\{$ Leff, $\delta, \lambda E, \lambda D\}$,

Leff $=7.1 ;(*$ K\&M 2003 Table $6 *)$

$\delta=$ Leff $* \mathrm{DD}[\mathrm{dp}] /$ Qaero;

$\lambda E=0.98 ;(\star K \& M 2003$ Table $6 *)$

$\lambda D:=\operatorname{If}[\delta>0.007,0.819 \operatorname{Exp}[-11.5 \delta]+0.0975 \operatorname{Exp}[-70.1 \delta]+$ $\left.0.0325 \operatorname{Exp}[-179 \delta], 1-5.50 \delta^{2 / 3}+3.77 \delta+0.814 \delta^{4 / 3}\right] ;$ $\lambda \mathrm{D} *$

$\lambda E]$

(* Elecrical mobility, C. $(\mathrm{m} / \mathrm{s}) / \mathrm{N} *$ )

zp [dia_, q_] $:=\frac{q e \operatorname{Cc}[\text { dia }]}{3 \pi \mu \text { dia }}$;

(* Centroid mobility*)

zpstar $\left[Q s_{-}, Q a_{-}, Q s_{-}, V_{-}\right]:=\frac{(2 Q s h+Q a-Q s)}{4 \pi V L D M A} \log [r 2 / r 1]$

$\Delta Z_{p s t a r}\left[Q s h, Q_{-}, Q_{-}, V_{-}\right]:=\frac{(Q \mathbf{a}+Q \mathbf{s})}{(2 Q s h+Q \mathbf{Q}-Q \mathbf{s})}$ zpstar [Qsh, Qa,Qs, v]

DMA=1 Voltages

res $=64 ;$

dpbin $=\operatorname{select}[$ Table [

Round $\left[10^{\log [10, \text { dplow }]+(i-1) / \text { res }}+\left(10 .^{\log [10, \text { dplow }]+(i) / \text { res }}-10^{\log [10, \text { dplow }]+(i-1) / \text { res }}\right) / 2, .01\right]$,

$\{1,1,200,1\}], \# \leq$ dphigh \&];

dpbinlimits $=$ Cases $\left[\right.$ Table $\left[\left\{\right.\right.$ Round $\left[10^{\log [10, \text { dplow }]+(i-1) / \text { res }}, .01\right]$, Round $\left[10^{\text {Log }[10, d p l o w]+(i) / r e s}\right.$, $.01]\},\{i, 1,200,1\}],\left\{x_{-} / ; x \mathbf{x} \leq\right.$ dphigh, yy_ $\left.\} \rightarrow\left\{\mathbf{x x}, y_{y}\right\}\right] ;$

(*determine voltage list for TDMA here, copy output below*) (*voltages $=$

Cases [Table [FindRoot [Zp [dpbin [ [i] ] $\left.10^{-9}, 1\right]=$ Zpstar [qsheath, qaero, qaero, V] , $\{\mathrm{V}, 100,1,10000\}][[1,2]],\{i$, Length $[$ dpbin $\left.\left.]\}], x x_{-} / ; x x \geq 5 \rightarrow x x\right] *\right)$ 

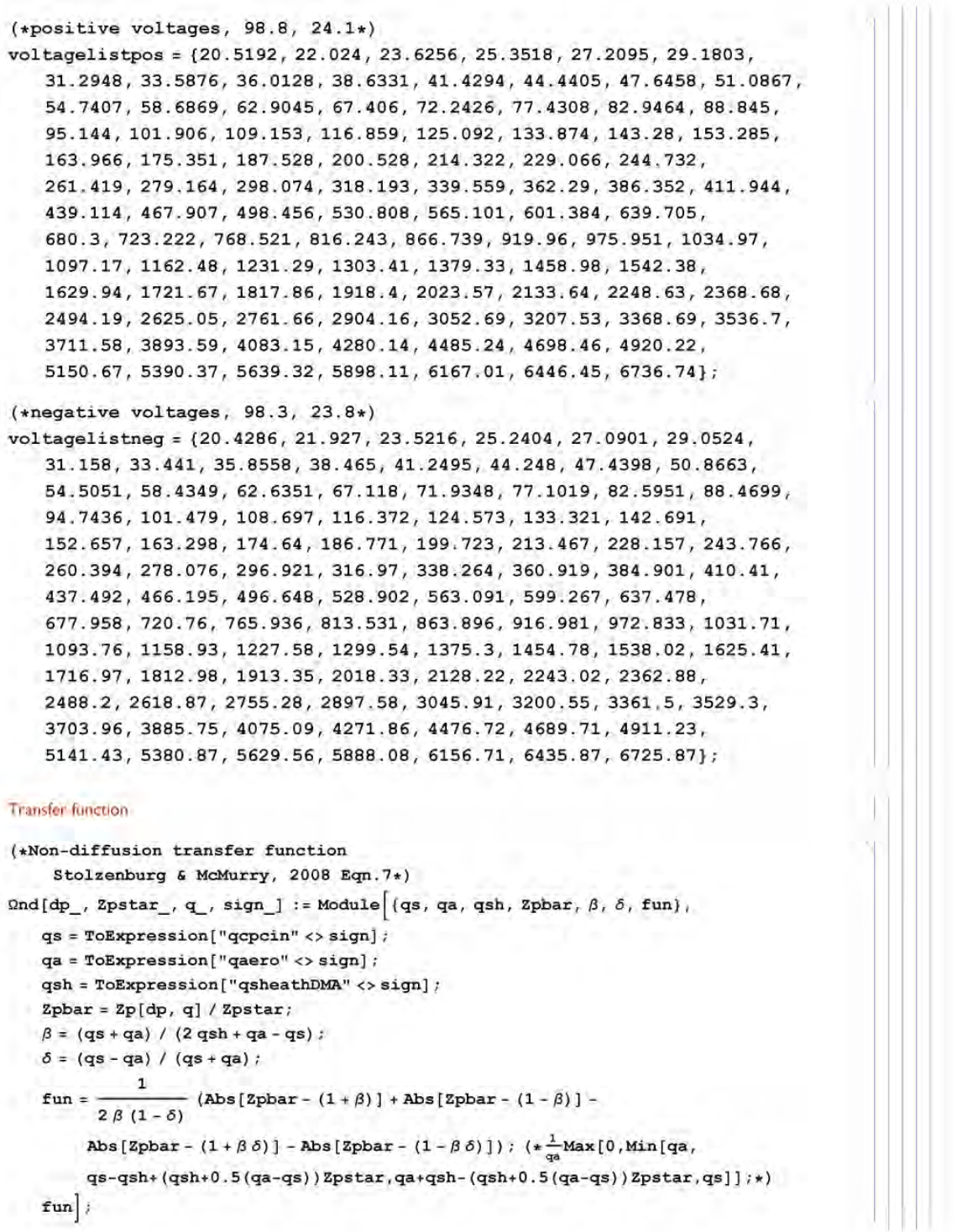

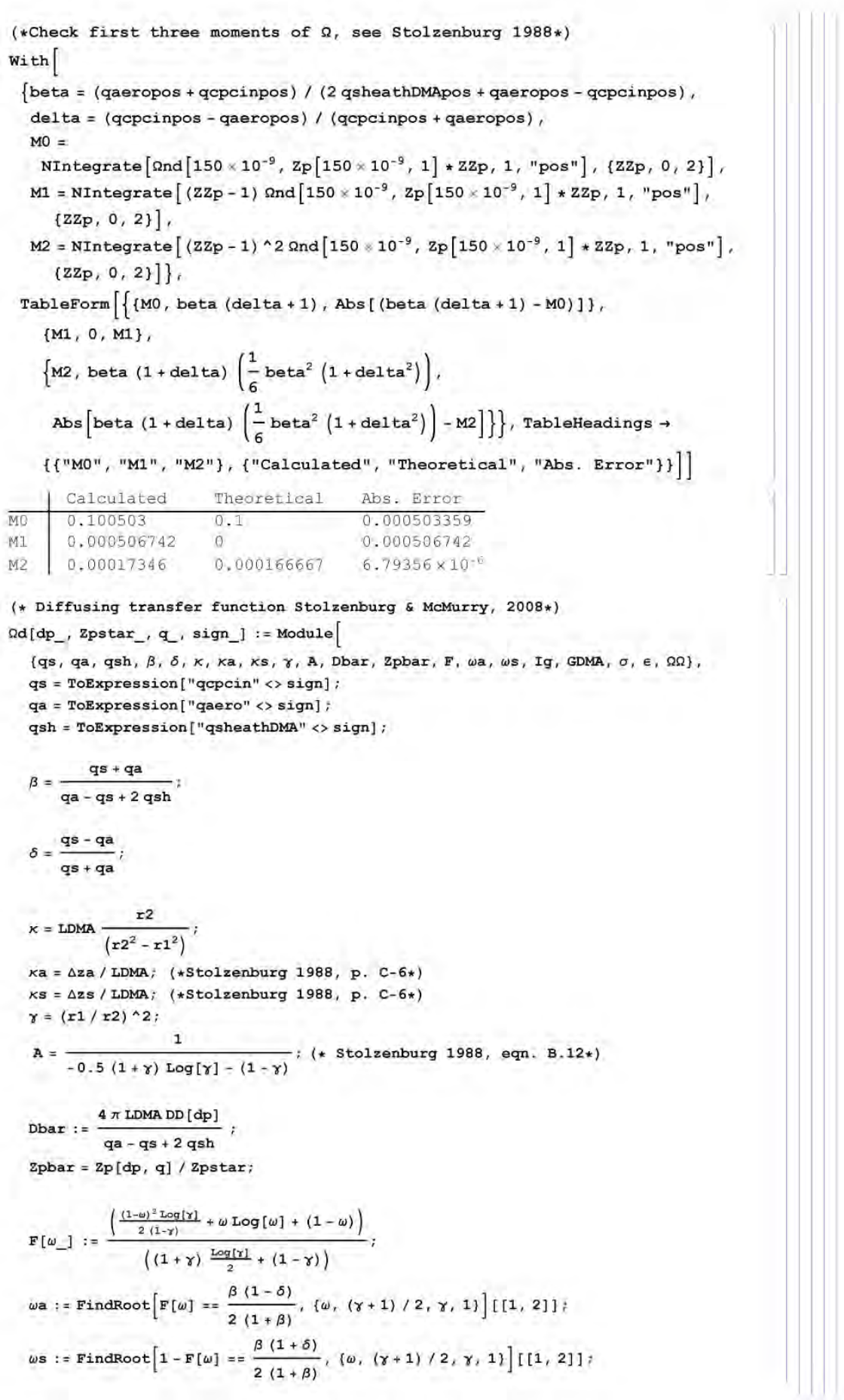


$$
\begin{aligned}
& \operatorname{Ig}\left[\omega_{-}\right]:=\frac{A^{2}}{(1-\gamma)}\left(-\omega^{2} \frac{((1-\gamma) \log [\omega]-(1-\omega) \log [\gamma])^{2}}{2}+\right. \\
& \left(\omega^{2} \frac{(1-\gamma)}{2}+\omega^{3} \frac{\log [\gamma]}{3}\right)((1-\gamma) \log [\omega]-(1-\omega) \log [\gamma])+ \\
& \left.\left(1-\omega^{2}\right) \frac{(1-\gamma)^{2}}{4}+5\left(1-\omega^{3}\right)(1-\gamma) \frac{\log [\gamma]}{18}+\left(1-\omega^{4}\right) \frac{(\log [\gamma])^{2}}{12}\right) ; \\
& \mathrm{GDMA}=\frac{4(1+\beta)^{2}}{(1-\gamma)}\left((\operatorname{Ig}[\omega \mathrm{s}]+\operatorname{Ig}[\omega \mathrm{a}])+\left(1+\left(\frac{\beta(1-\delta)}{\kappa a}\right)\left(\frac{1-\omega \mathrm{a}}{1-\gamma}\right)+\left(\frac{\beta(1+\delta)}{\kappa \mathrm{s}}\right)\left(\frac{\omega \mathrm{s}-\gamma}{1-\gamma}\right)\right)\right. \\
& \left.\frac{1}{(2(1+\beta) \kappa)^{2}}\right) \text {; } \\
& (* G=2.14 \text { for TSI } 3081 \text {, BW P. } 559 \text { (different definition?)*) ; }
\end{aligned}
$$

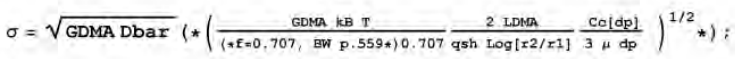

$$
\begin{aligned}
& \in\left[x_{-}\right]:=x \operatorname{Erf}[x]+\operatorname{Exp}\left[-x^{2}\right] / \sqrt{\pi} ; \\
& \Omega \Omega=\frac{\sigma}{\sqrt{2} \beta(1-\delta)} \\
& \left(\epsilon\left[\frac{\text { Zpbar }-(1+\beta)}{\sqrt{2} \sigma}\right]+\epsilon\left[\frac{\text { zpbar }-(1-\beta)}{\sqrt{2} \sigma}\right]-\epsilon\left[\frac{\text { zpbar }-(1+\beta \delta)}{\sqrt{2} \sigma}\right]-\epsilon\left[\frac{\text { zpbar }-(1-\beta \delta)}{\sqrt{2} \sigma}\right]\right) ; \\
& \Omega \Omega] \text {; }
\end{aligned}
$$

(*Check first three moments of $\Omega$, see Stolzenburg 1988*) with [

(beta $=$ (qaeropos + qcpcinpos) $/(2$ qsheathDMApos + qaeropos -qcpcinpos $)$, delta $=$ (qcpcinpos - qaeropos) $/$ (qcpcinpos + qaeropos $),$

MO $=$

NIntegrate $\left[\Omega \mathrm{d}\left[550 \times 10^{-9}, \mathrm{zp}\left[550 \times 10^{-9}, 1\right] * \mathrm{ZZp}, 1, " \mathrm{pos} "\right],\{\mathrm{zZp}, 0,2\}\right]$ ， $\mathrm{MI}=\operatorname{NIntegrate}\left[(\mathrm{zZp}-1) \Omega \mathrm{d}\left[550 \times 10^{-9}, \mathrm{z}_{\mathrm{P}}\left[550 \times 10^{-9}, 1\right] * \mathrm{zZ}_{\mathrm{P}}, 1\right.\right.$, "pos" $]$, $\{z z p, 0,2\}]$,

$\mathrm{M} 2=\mathrm{NIntegrate}\left[\left(\mathrm{zz}_{\mathrm{p}}-1\right)^{\wedge} 2 \Omega \mathrm{d}\left[550 \times 10^{-9}, \mathrm{zp}\left[550 \times 10^{-9}, 1\right] \star \mathrm{zz}_{\mathrm{p}}, 1, " \mathrm{pos} "\right]\right.$, $\{\mathrm{zzp}, 0,2\}]\}$,

TableForm $[\{$ MO, beta $(\operatorname{delta}+1)$, Abs $[($ beta $(\operatorname{delta}+1)-M 0)]\}$,

$\{\mathrm{M} 1,0, \mathrm{M} 1\}$,

$\left\{\right.$ M2, beta $(1+\operatorname{delta})\left(\frac{1}{6} \operatorname{beta}^{2}\left(1+\operatorname{delta}^{2}\right)\right)$.

Abs $\left.\left.\left[\operatorname{beta}(1+\operatorname{delta})\left(\frac{1}{6} \operatorname{beta}^{2}\left(1+\operatorname{delta}^{2}\right)\right)-\mathrm{M} 2\right]\right\}\right\}$, TableHeadings $\rightarrow$

$\{\{$ "MO", "M1", "M2"\}, \{"Calculated", "Theoretica1", "Abs. Error" \}\}]]

\begin{tabular}{l|lll} 
& Calculated & Theoretical & Abs. Error \\
\hline M0 & 0.100507 & 0.1 & 0.00050741 \\
M1 & 0.000510862 & 0 & 0.000510862 \\
M2 & 0.000174926 & 0.000166667 & $8.2594 \times 10^{-5}$
\end{tabular}

Particle Charging

Boltzmann Charge distribution

$f B\left[n_{-}, d_{-}\right]:=\left(\frac{\left(9.0 \times 10^{9}\right) e^{2}}{\left(\pi \mathrm{dp} 10^{-9} \mathrm{kB} \mathrm{T}\right)}\right)^{0.5} \operatorname{Exp}\left[-\frac{\left(9.0 \times 10^{9}\right) \mathrm{n}^{2} \mathrm{e}^{2}}{\mathrm{dp} 10^{-9} \mathrm{kB} \mathrm{T}}\right]$

Wiedensohler (1988) approximation to Fuch's (corrected) diffusion theory (see TSI SMPS or 3080 manuals and "Aerosol Measurement $2^{\text {nd }}$ ed." by Baron \& Willeke p. 547 
$\mathrm{fW}\left[\mathrm{n}_{-}, \mathrm{dp}\right]:=10\left(\sum_{i=0}^{5} \mathrm{a}[\mathrm{i}][\mathrm{n}](\log [10, \mathrm{dp}])^{2}\right) / ; \operatorname{Abs}[\mathrm{n}] \leq 2$

$f \mathrm{~W}\left[\mathrm{n}_{-}, \mathrm{dp}_{-}\right]:=$

$\frac{e}{\sqrt{4 \pi^{2} \in 0 d p\left(10^{-9}\right) k B T}} \operatorname{Exp}\left[\frac{-\left(n-\frac{2 \pi \epsilon 0 d p(10.9) k B T}{e^{2}} \log [\text { ziratio }]\right)^{2}}{2 \frac{2 \pi \epsilon 0 d p\left(10^{-9}\right) k B T}{e^{2}}}\right] ;$ Abs $[n]>2$

$\left(*\right.$ Wiedensohler $\alpha_{i, k}$ coefficients, note $a[4][1] \& a[5][2]$

are different from originally published in 1988 (B\&W p. 547)*)

$\mathrm{a}[0][\mathrm{n}] \mathrm{:}=\{-26.3328,-2.3197,-0.0003,-2.3484,-44.4756\}[[\mathrm{n}+3]]$;

$\left.\mathrm{a}[1]\left[\mathrm{n}_{-}\right]:=\{35.9044,0.6175,-0.1014,0.6044,79.3772\}[\mathrm{n}+3]\right]$;

$a[2]\left[n_{-}\right]:=\{-21.4608,0.6201,0.3073,0.4800,-62.89\}[[n+3]]$;

$\left.a[3]\left[n_{-}\right]:=\{7.0867,-0.1105,-0.3372,0.0013,26.4492\}[n+3]\right]$;

$\mathrm{a}[4]\left[\mathrm{n}_{-}\right]:=\{-1.3088,-0.1260,0.1023,-0.1553,-5.7480\}[[\mathrm{n}+3]]$;

$a[5]\left[n_{-}\right]:=\{0.1051,0.0297,-0.0105,0.0320,0.5049\}[[n+3]]$;

ziratio $=0.875 ;\left(* \mathrm{z}_{i_{+}} / \mathrm{z}_{\mathrm{i}_{-} *}\right)$

$\log \log p 1 \circ t[\{\mathrm{fw}[0, d p], f w[1, d p], f w[-1, d p], f W[2, d p], f w[-2, d p]$,

$f W[3, d p], f W[-3, d p], f B[0, d p], f B[1, d p], f B[2, d p], f B[3, d p]\}$,

$\{d p, 2,1000\}$, PlotRange $\rightarrow\left\{\right.$ Automatic, $\left.\left\{10^{-3}, 2\right\}\right\}$, Frame $\rightarrow$ True,

FrameLabel $\rightarrow\{$ "dp $(\mathrm{nm}) ", " f[\mathrm{~N}] "\}$, PlotStyle $\rightarrow$ \{Directive[Black], Directive[Blue],

Directive[Blue, Dashed], Directive[Green], Directive[Green, Dashed]

Directive[Red], Directive[Red, Dashed], Directive[Black, Dotted],

Directive [Blue, Dotted], Directive[Green, Dotted], Directive[Red, Dotted]\},

PlotIegends $\rightarrow$ Flatten [Append [Table [ $q="\langle>$ ToString [i] , $\{i,-3,3,1\}]$,

$\{" q B=0 ", " q B= \pm 1 ", " q B= \pm 2 ", " q B= \pm 3 "\}]\}]$

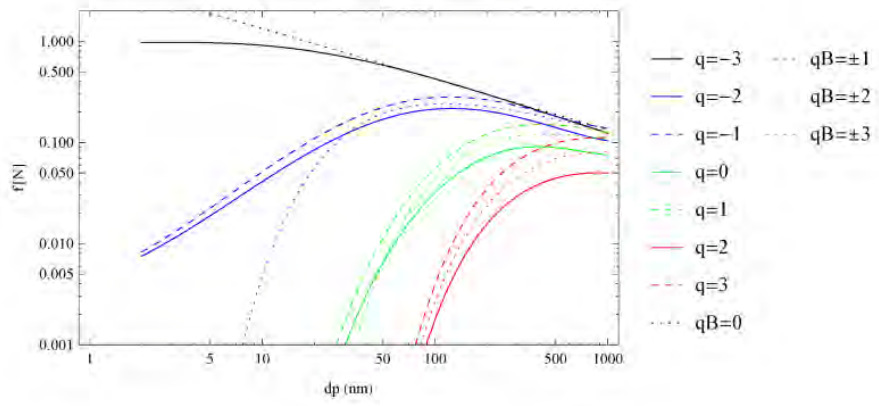

Mulciple Charge Correction

dpbin $=$ Select $[$ Table [

Round $\left[10^{\log [10, \text { dplowpos }]+(1-1) / 64}+\left(10 .^{\operatorname{tog}[10, \text { dplowpos }]+(1) / 64}-10^{\log [10, \text { dplowpos }]+(1-1) / 64}\right) / 2\right.$,

$.01],\{i, 1,200,1\}], \# \leq$ dphighpos \&];

dpbinlimits $=$ Cases $\left[\right.$ Table $\left[\left\{\right.\right.$ Round $\left[10^{\log [10, \text { dplowpos }]+(i-1) / 64}, .01\right]$,

Round $\left.\left.\left[10^{\log [10, \text { dpiompos }]+(i) / 64}, .01\right]\right\},\{i, 1,200,1\}\right]$,

$\left\{\mathbf{x x} /\right.$; Round $[\mathbf{x x}, 1] \leq$ dphighpos, $\left.\left.\mathbf{Y Y}_{-}\right\} \rightarrow\{\mathbf{x x}, \mathbf{Y Y}\}\right]$;

(*find the particle size dp2 associated with actual charge q2,

for indicated size $d p 1$ and charge $q 1 *)$

qcorr $\left.\left[\mathrm{dp} 1_{-}, \mathrm{q}_{-}{ }^{\prime} \mathrm{q}_{-}\right]\right]:=\operatorname{Module}[\{\mathrm{dp} 2\}$,

$d p 2=$ FindRoot $[d d p / C c[d d p]==(d p 1 / C c[d p 1])(q 1 / q 2)$,

$\{$ ddp, dp1\}, AccuracyGoal $\rightarrow \infty][[1,2]] / /$ quiet;

dp2] 


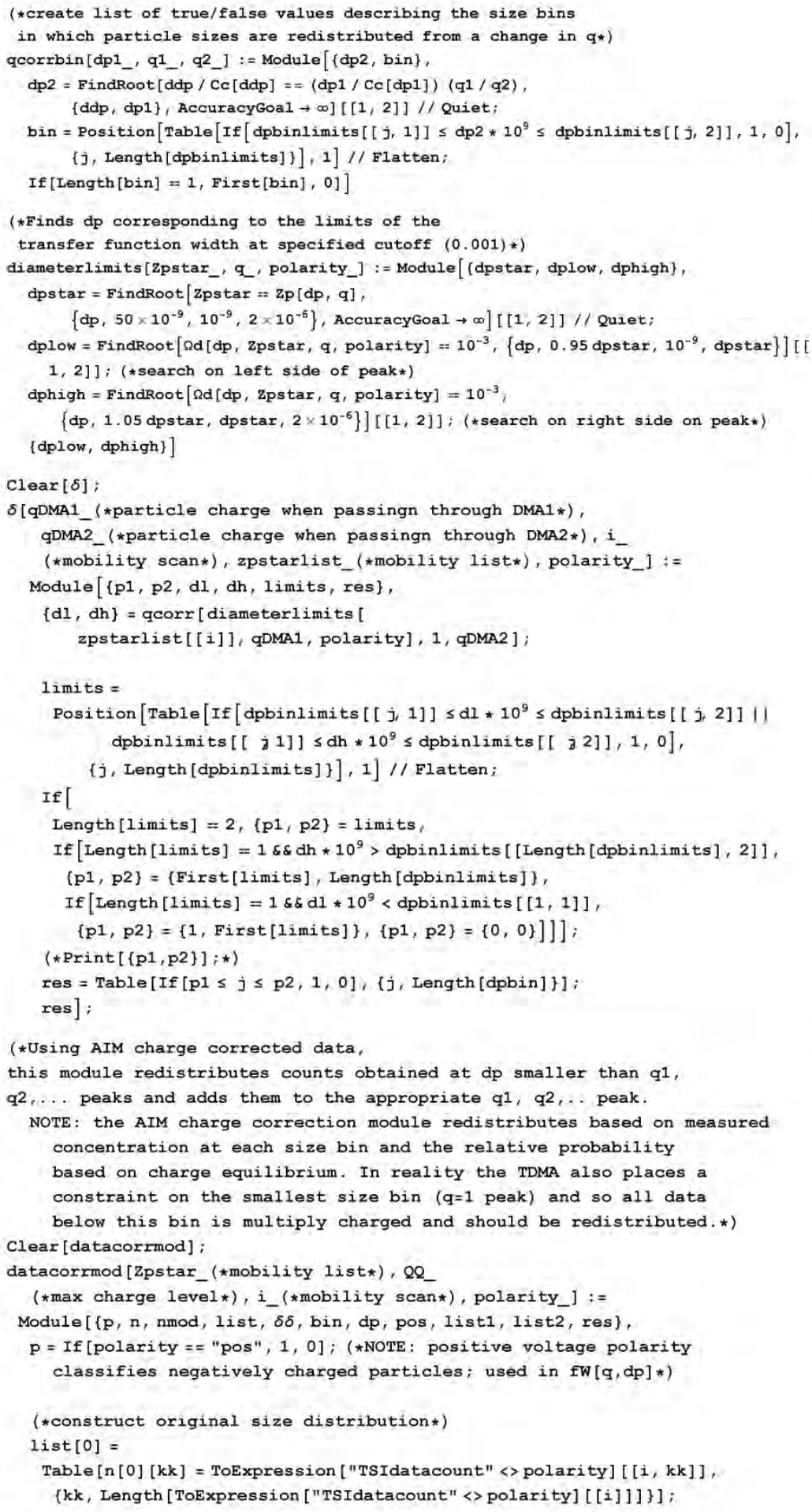




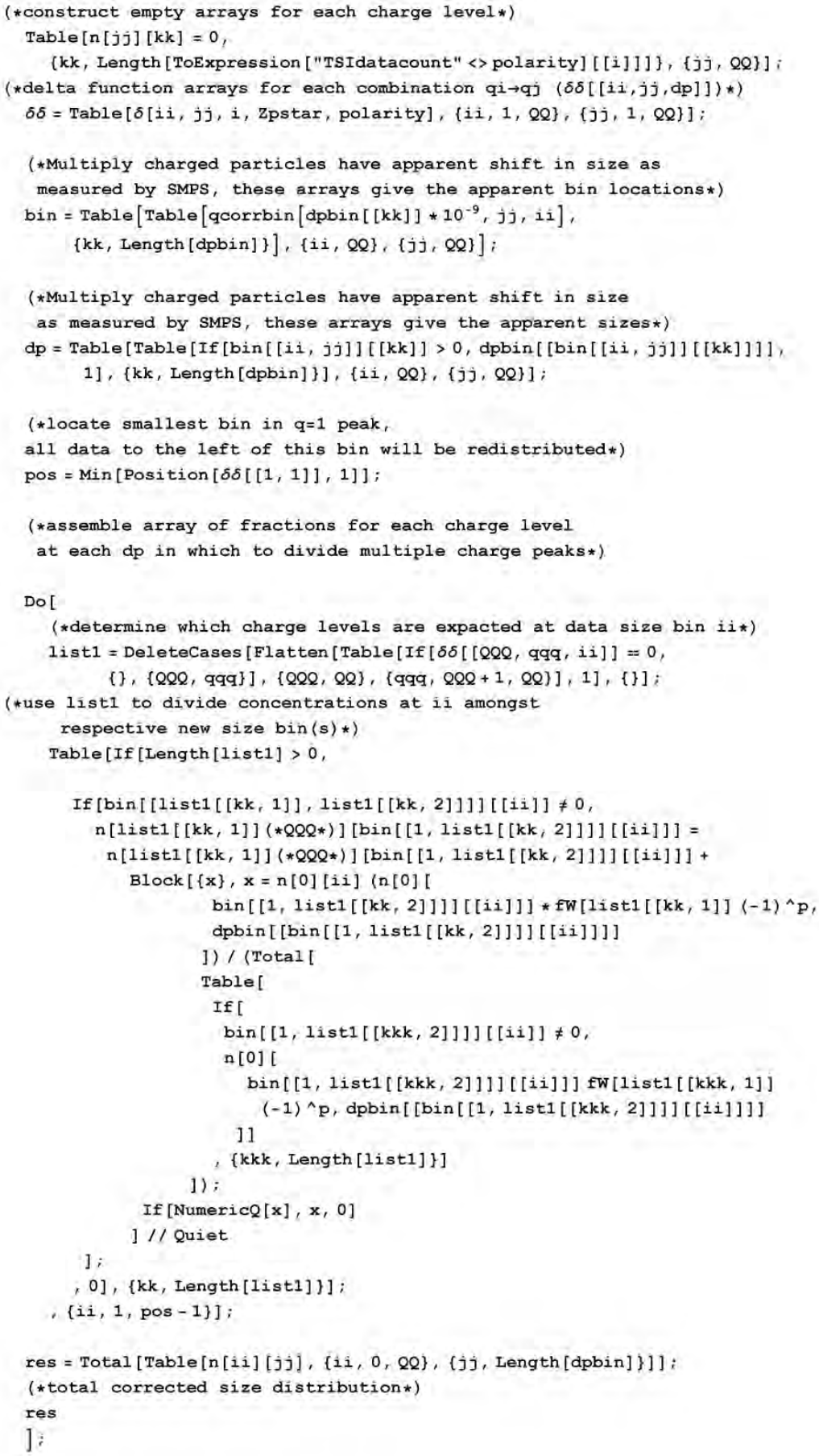




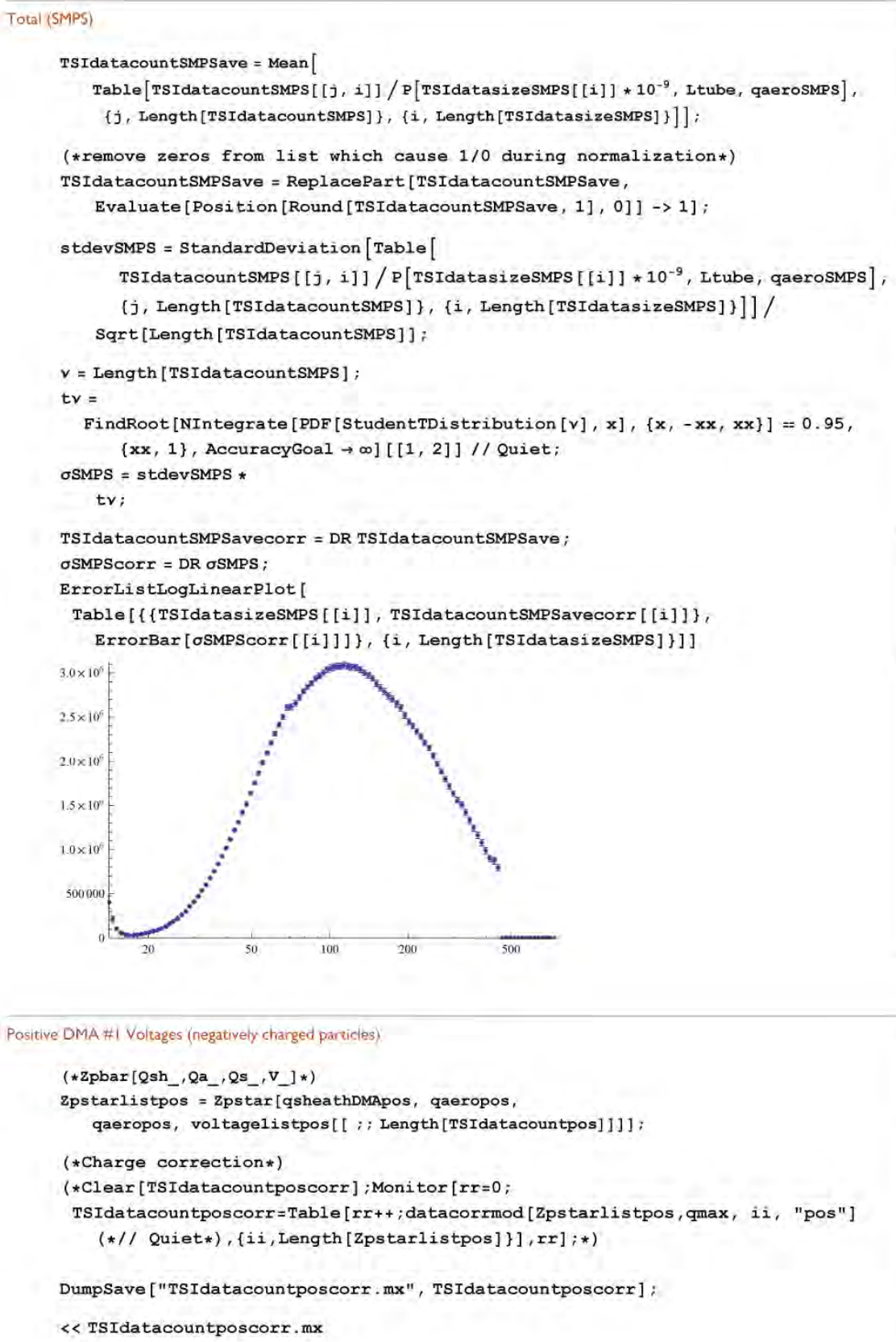

Positive DMA \#1 Voltages (negatively changed paricies).

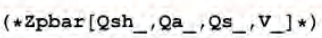

zpstarlistpos = zpstar[qsheathDMApos, qaeropos,

qaeropos, voltagelistpos[[ ; Length[TSIdatacountpos]]]] ;

(*Charge correction*)

( $*$ Clear [TSIdatacountposcorr] ; Monitor $[r r=0$;

TSIdatacountposcorr=Table [rr++; datacorrmod [Zpstarlistpos, qmax, ii, "pos"]

(*// Quiet*), \{ii, Length [Zpstarlistpos] $\}], r r] ; *)$

DumpSave ["TSIdatacountposcorr.mx", TSIdatacountposcorr] ;

$<$ TSIdatacountposcorr.mx 


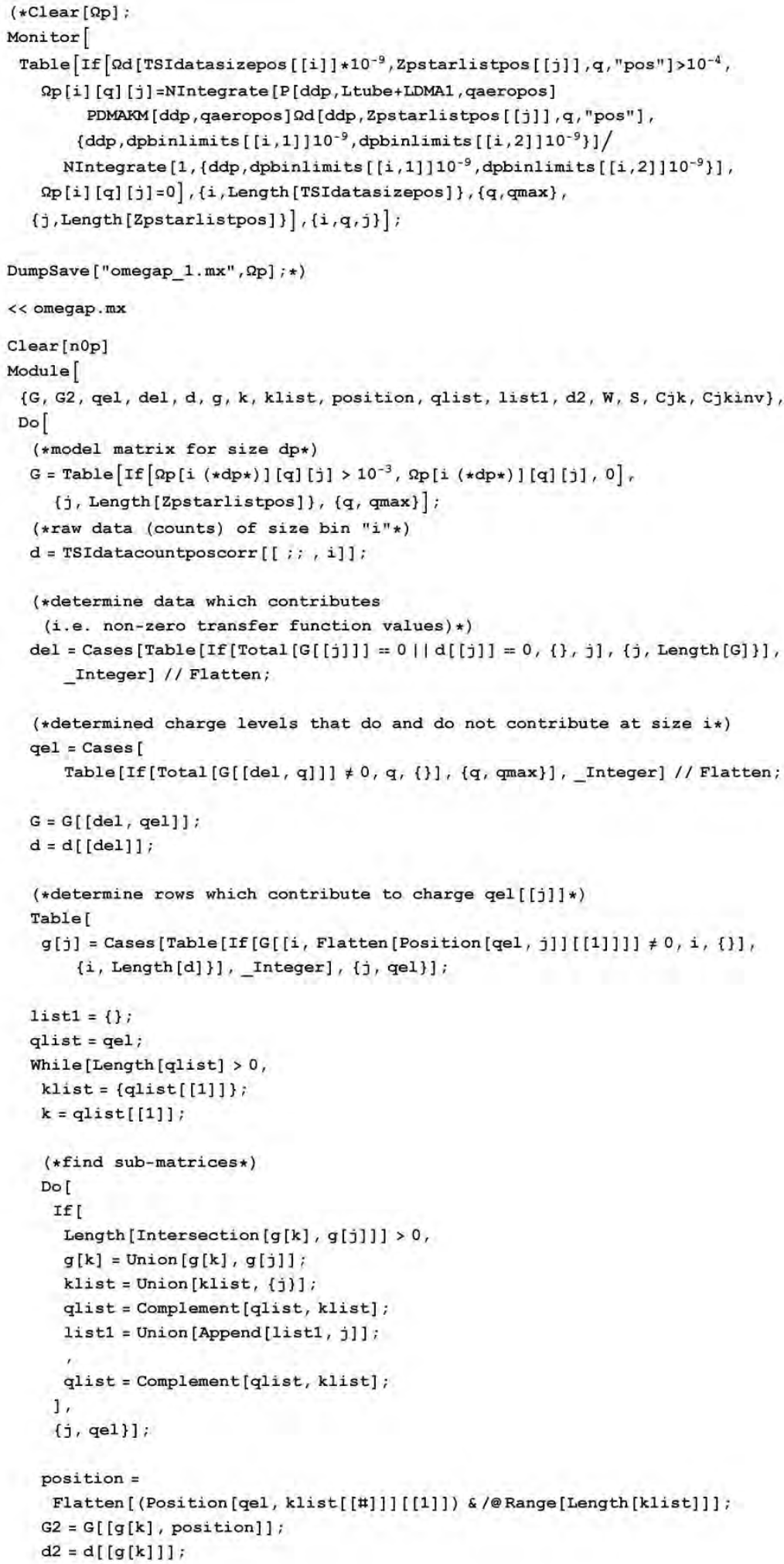




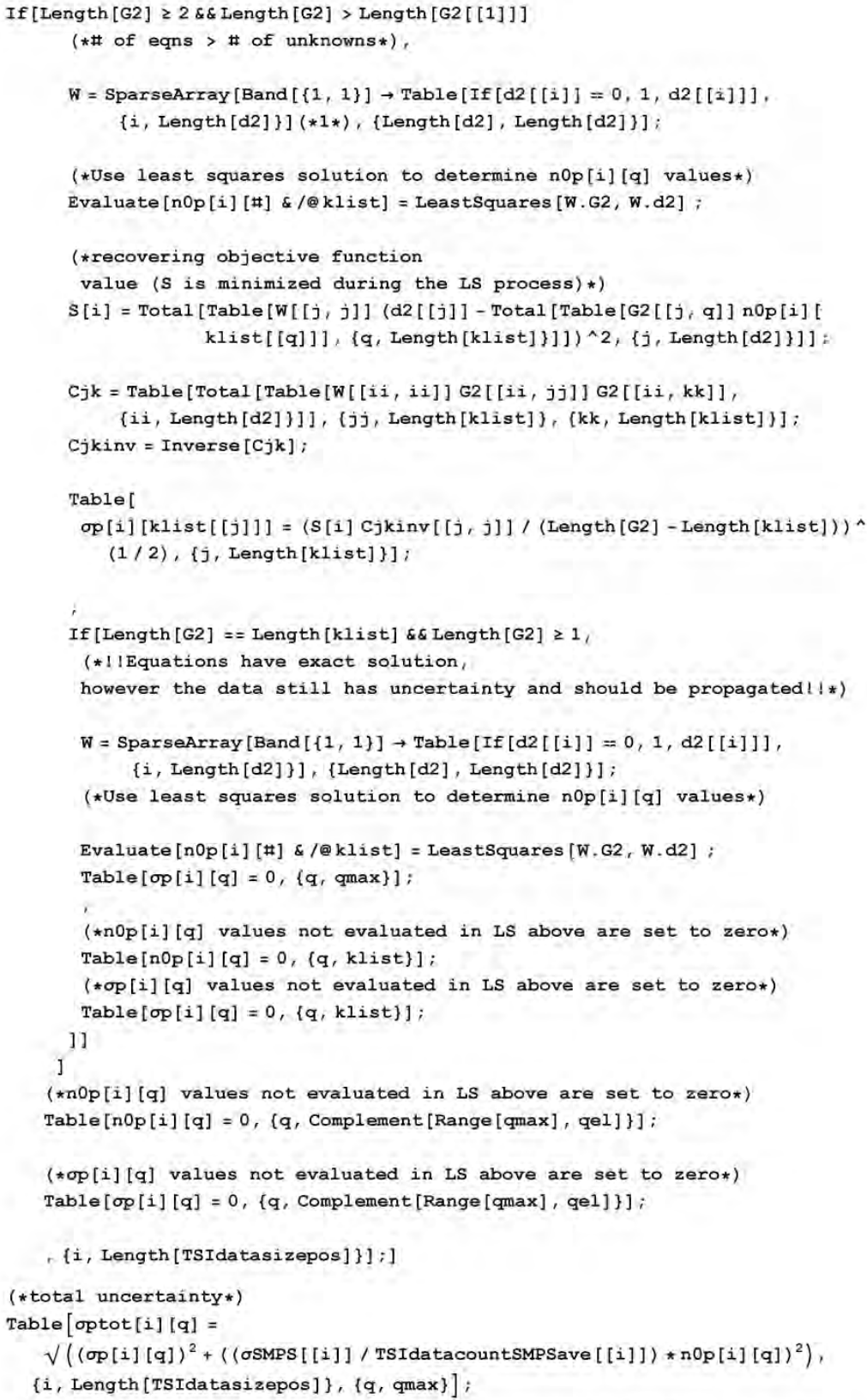

Table $[\mathrm{n} 0 \mathrm{pcorr}[i][\mathrm{q}]=\mathrm{DR}$ n0p $[i][\mathrm{q}],\{\mathrm{q}, \mathrm{qmax}\},\{i, \operatorname{Length}[\mathrm{TSIdatasizepos}]\}]$; Table $[$ optotcorr $[i][q]=\operatorname{DR}$ optot $[i][q],\{q, q \operatorname{qmax}\},\{i$, Length $[$ TSIdatasizepos $]\}]$; 
Show $[\{$ ErrorListLogLogPlot [

Table [ \{ $\{$ TSIdatasizepos [ [i]], n0pcorr [i] [1]\}, ErrorBar [optotcorr [i] [1]]\},

$\{i$, Length [TSIdatasizepos $]\}]$, PlotRange $\left.\rightarrow\left\{\{5,800\},\left\{100,10^{6}\right\}\right\}\right]$,

ErrorListLogLogPlot [Table [\{ \{TSIdatasizepos [[i]], n0pcorr[i] [2]\},

ErrorBar [optotcorr [i] [2]]\}, \{i, Length [TSIdatasizepos] $\}]$,

PlotRange $\left.\rightarrow\left\{\{5,800\},\left\{100,10^{6}\right\}\right\}\right]$, ErrorListLogLogPlot [

Table [\{ \{TSIdatasizepos [ [i]], nopcorr [i] [3] \}, ErrorBar [optotcorr [i] [3]] \},

$\{i$, Length [TSIdatasizepos] $\}]$, PlotRange $\left.\left.\left.\rightarrow\left\{\{5,800\},\left\{100,10^{6}\right\}\right\}\right]\right\}\right]$

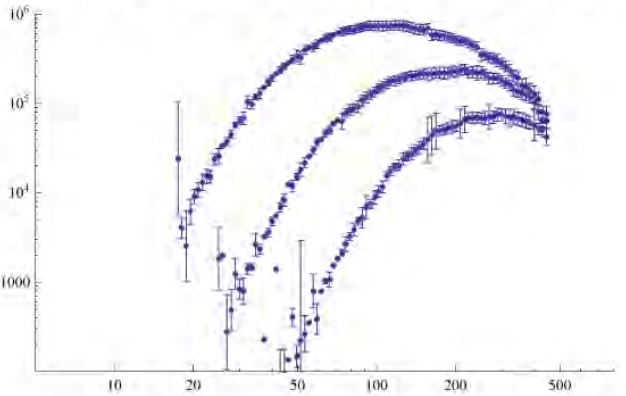

Negative DMA \#1 Voltages (positively charged particles)

( Z Zpbar $\left.\left[Q s h_{-}, Q a_{-}, Q s_{-}, v_{-}\right] *\right)$

zpstarlistneg = zpstar [qsheathDMAneg, qaeroneg,

qaeroneg, voltagelistneg[ [; Length [TSIdatacountneg] ]]] ;

(*charge correction*)

( $*$ Clear [TSIdatacountnegcorr] :

Monitor $[\mathrm{rr}=0$;

TSIda tacountnegcorr $=$ Table [rr++; datacorrmod [Zpstarlistneg, qmax, il, "neg"] ,

$\{i 1$, Length [Zpstarlistneg] $\}], \mathrm{rr}] ; *$ )

DumpSave ["TSIdatacountnegcorr_1.mx", TSIdatacountnegcorr] ;

$\ll$ TSIdatacountnegcorr.mx

(*Using average value of $\Omega d[]$ over size interval*)

$(*$ Clear $[\Omega \mathrm{n}]$;

Monitor [

Table [If [ $\Omega$ d [TSIdatasizeneg [ [i]] $* 10^{-9}$, zpstarlistneg [ [j]] , q, "neg"] $>10^{-4}$,

Sn [i] [q] [j] =NIntegrate [P [ddp, Ltube+LDMA1, qaeroneg]

PDMAKM [ddp, qaeroneg] $\Omega d$ [ddp, zpstar1 istneg [ [j]] , q, "neg"] ,

$\left\{d d p\right.$, dpbinlimits $[[i, 1]] 10^{-9}$, dpbinlimits $\left.\left.\left.[i, 2]\right] 10^{-9}\right\}\right] /$

NIntegrate $\left[1,\left\{\right.\right.$ ddp, dpbinlimits $[[i, 1]] 10^{-9}$, dpbinlimits $\left.\left.[[i, 2]] 10^{-9}\right\}\right]$,

$\Omega n[i][q][j]=0],\{i$, Length [TSIdatasizeneg] $\},\{q, q \max \}$,

$\{j$, Length [Zpstarlistneg] $\}],\{i, q, j\}]$;

DumpSave ["omegan_1.mx", $\Omega \mathrm{n}] ; *$ )

$<$ omegan.mx

Clear [nOn]

Module [

$\{G, G 2, q e 1$, del, d, g, k, klist, position, qlist, list1, d2, w, S, Cjk, Cjkinv\}, Do [

(*model matrix for size dp*)

$G=\operatorname{Table}\left[\operatorname{If}\left[\Omega \operatorname{R}[i(\star d p *)][q][j]>10^{-3}, \operatorname{\Omega n}[i(* d p *)][q][j], 0\right]\right.$,

$\{j$, Length [Zpstarlistneg]\}, \{q, qmax $\}]$;

(*raw data (counts) of size bin "i"*)

$\mathrm{d}=$ TSIdatacountnegcorr $[[; ;, i]]$; 


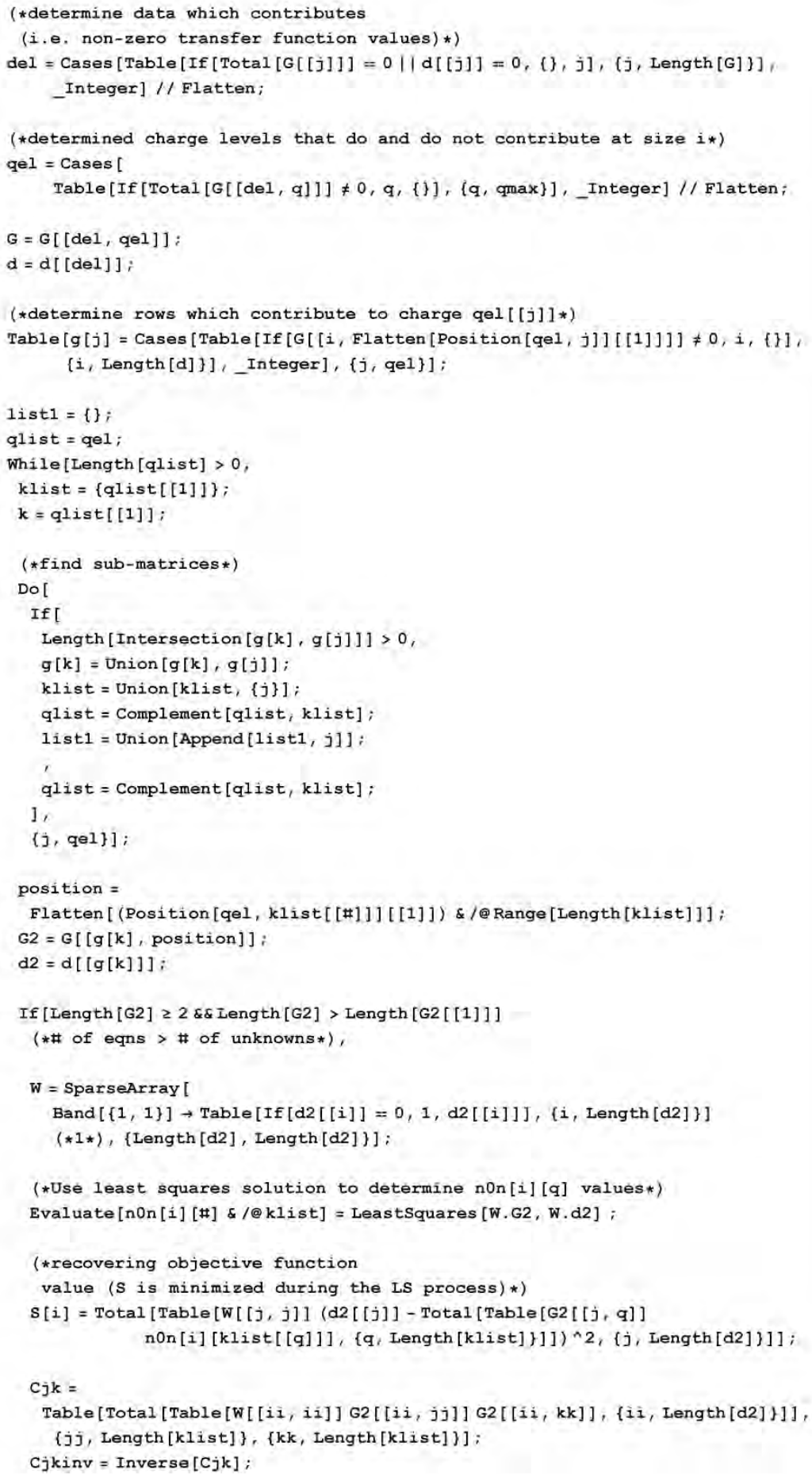



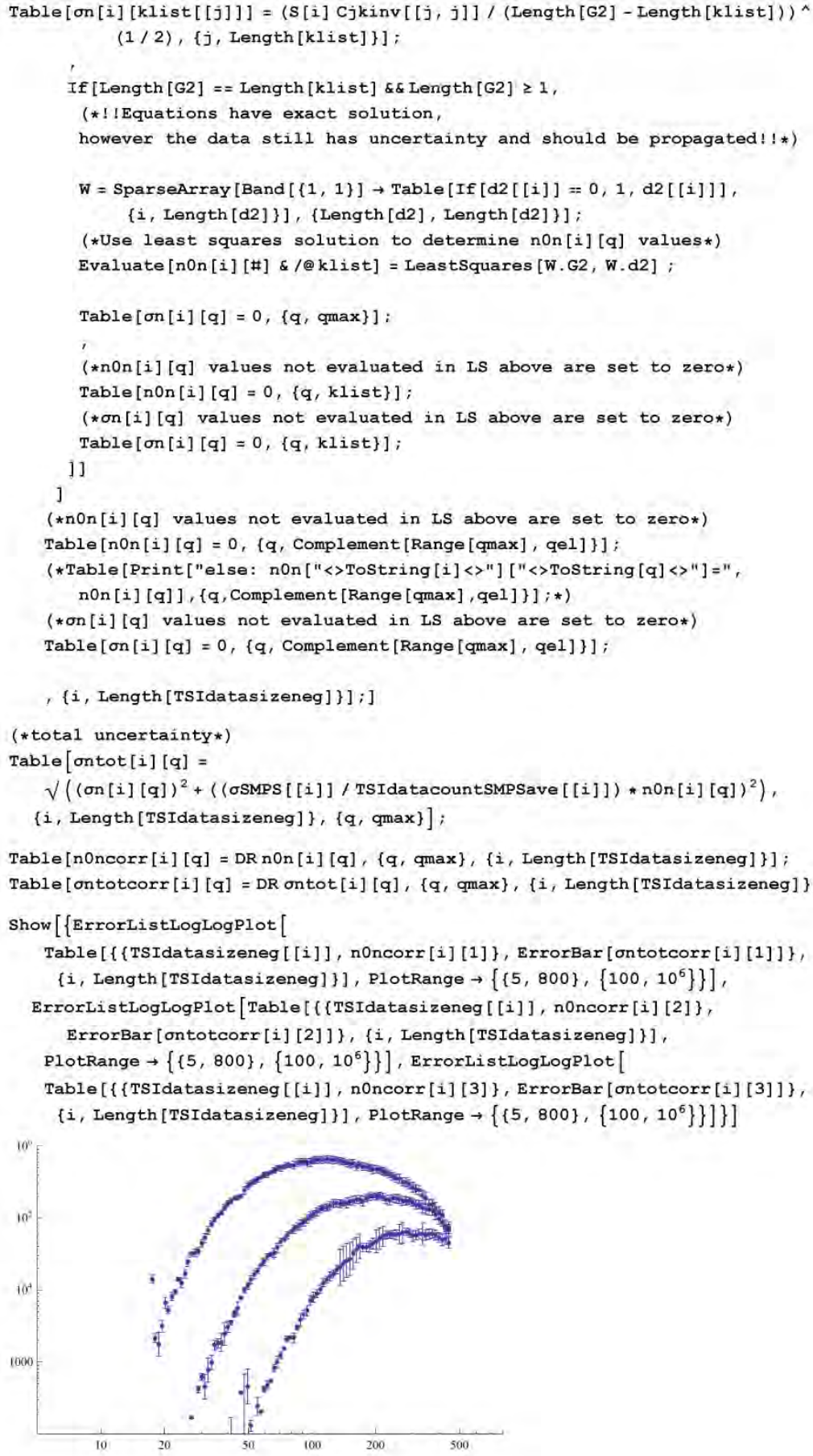
Charge Distribution

n00corr $=$ Table $[\mathrm{TSI}$ datacountSMPSavecorr $[[i]]-\operatorname{Total}[\mathrm{Tab} l e[\mathrm{n} 0 \mathrm{ncorr}[i][q],\{q, q \max \}]]-$ Total [Table [n0pcorr $[i][q],\{q, q \max \}]],\{i$, Length [TSIdatasizepos] $\}]$;

$\operatorname{Table}\left[\sigma 0 \operatorname{tot}[i]=\sqrt{ }\left((\sigma \operatorname{SMPSCorr}[[i]])^{2}+\operatorname{Total}[\operatorname{Table}[\sigma \mathrm{ntotcorr}[i][q] \wedge 2,\{q, q \max \}]]+\right.\right.$ Total [Table $[$ optotcorr $[i][q] \wedge 2,\{q, q \max \}]]),\{i$, Length [TSIdatasizeneg] $\}]$;

Show [ $\{$ ListLogLogPlot [

Table[\{TSIdatasizeneg[i]], n0ncorr[i] [q] / TSIdatacountSMPSavecorr[ii]]\},

$\{q, 4\},\{i, 1$, Length [TSIdatasizeneg] $\}]$,

PlotRange $\rightarrow\{\{1,2000\},\{0.001,1\}\}$, Joined $\rightarrow$ False,

Plotstyle $\rightarrow$ \{Blue, Red, Green, Cyan $\}$,

ListLogLogPlot [Table[\{TSIdatasizepos[[i]], nopcorr[i] [q] /

TSIdatacountSMPSavecorr $[[i]]\},\{q, 4\},\{i, 1$, Length [TSIdatasizepos] $\}]$,

PlotRange $\rightarrow\{\{1,2000\},\{0.001,1\}\}$, Joined $\rightarrow$ False, PlotMarkers $\rightarrow$ "0",

Plotstyle $\rightarrow$ \{Blue, Red, Green, Cyan $\}$,

ListLogLogPlot [Table [ \{TSIdatasizeSMPS[[i]],

n00corr [[i]] / TSIdatacountSMPSavecorr[[i]]\},

$\{i, 1$, Length [TSIdatasizeSMPS $]\}]$, PlotStyle $\rightarrow$ Orange $]$,

$\log \log P \operatorname{lot}[(\mathrm{fW}[0, d p], f B[0, d p], f W[1, d p], f W[-1, d p], f B[1, d p], f W[2, d p]$, $f W[-2, d p], f B[2, d p], f W[3, d p], f W[-3, d p], f B[3, d p], f W[4, d p]$,

$f W[-4, d p], f B[4, d p]\},\{d p, 5,1200\}, P l o t R a n g e \rightarrow\{(1,2000\},\{0.001,1\}\}$

Plotstyle $\rightarrow$ [Directive [Orange], Directive [Orange, Dotted],

Directive[Blue], Directive[Blue, Dashed], Directive[Blue, Dotted],

Directive[Red], Directive[Red, Dashed], Directive[Red, Dotted],

Directive [Green], Directive[Green, Dashed], Directive[Green, Dotted],

Directive[Cyan], Directive [Cyan, Dashed], Directive[Cyan, Dotted] \}] \}]
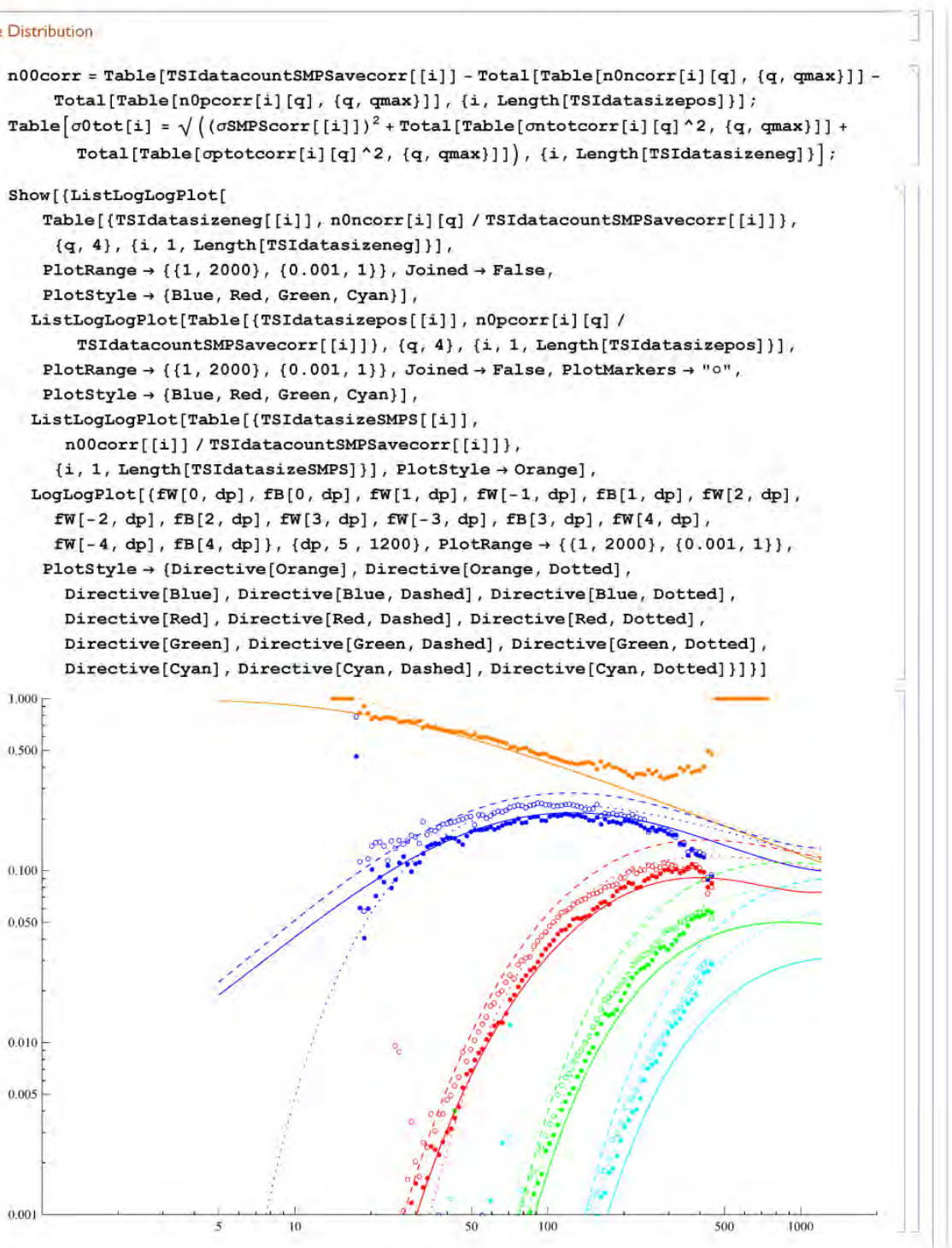
Show [ $\{$ ErrorListLogLogPlot [

Table [ \{ $\{$ TSIdatasizeneg [i]], nOncorr [i] [q] / TSIdatacountSMPSave [ii]]\}, ErrorBar [ontotcorr[i] [q] / TSIdatacountSMPSave[[i]]]\},

$\{q, 4\},\{i, 1$, Length [TSIdatasizeneg] $\}]$,

PlotRange $\rightarrow\{\{1,2000\},\{0.001,1\}\}$, Joined $\rightarrow$ False,

Plotstyle $\rightarrow\{$ Blue, Red, Green, Cyan $\}]$,

ErrorListLogLogPlot [Table [

$\{\{$ TSIdatasizepos [ [i]] , n0pcorr [i] [q] / TSIdatacountSMPSave [[i]]\},

ErrorBar [optotcorr [i] [q] / TSIdatacountSMPSave $[i]]]\},\{q, 4\}$,

$\{i, 1$, Length $[$ TSIdatasizepos $]\}]$, PlotRange $\rightarrow\{\{1,2000\},\{0.001,1\}\}$,

Joined $\rightarrow$ False, PlotMarkers $\rightarrow$ "o", PlotStyle $\rightarrow$ \{Blue, Red, Green, Cyan $\}]$,

ErrorListLogLogPlot [Table [

$\{\{$ TSIdatasizeSMPS [[i]], n00corr[[i]] / TSIdatacountSMPSave [[i]]\},

ErrorBar [ $\sigma 0$ tot $[i] /$ TSIdatacountSMPSave [i] $]]\}$,

$\{i, 1$, Length [TSIdatasizeSMPS $]\}]$, PlotStyle $\rightarrow$ Orange $]$,

$\log \log P \operatorname{lot}[\{\mathrm{fW}[0, d p], f B[0, d p], f W[1, d p], f W[-1, d p], f B[1, d p], f W[2, d p]$ $\mathrm{fW}[-2, d p], f B[2, d p], f W[3, d p], f W[-3, d p], f B[3, d p], f W[4, d p]$,

$\mathrm{fW}[-4, d p], \mathrm{fB}[4, \mathrm{dp}]\},\{d p, 5,1200\}$, PlotRange $\rightarrow\{\{1,2000\},\{0.001,1\}\}$,

Plotstyle $\rightarrow$ \{Directive [Orange], Directive [Orange, Dotted],

Directive [Blue], Directive [B1ue, Dashed], Directive[Blue, Dotted],

Directive [Red], Directive [Red, Dashed], Directive[Red, Dotted],

Directive [Green], Directive [Green, Dashed], Directive [Green, Dotted],

Directive [Cyan], Directive [Cyan, Dashed], Directive [Cyan, Dotted]\}] \}]
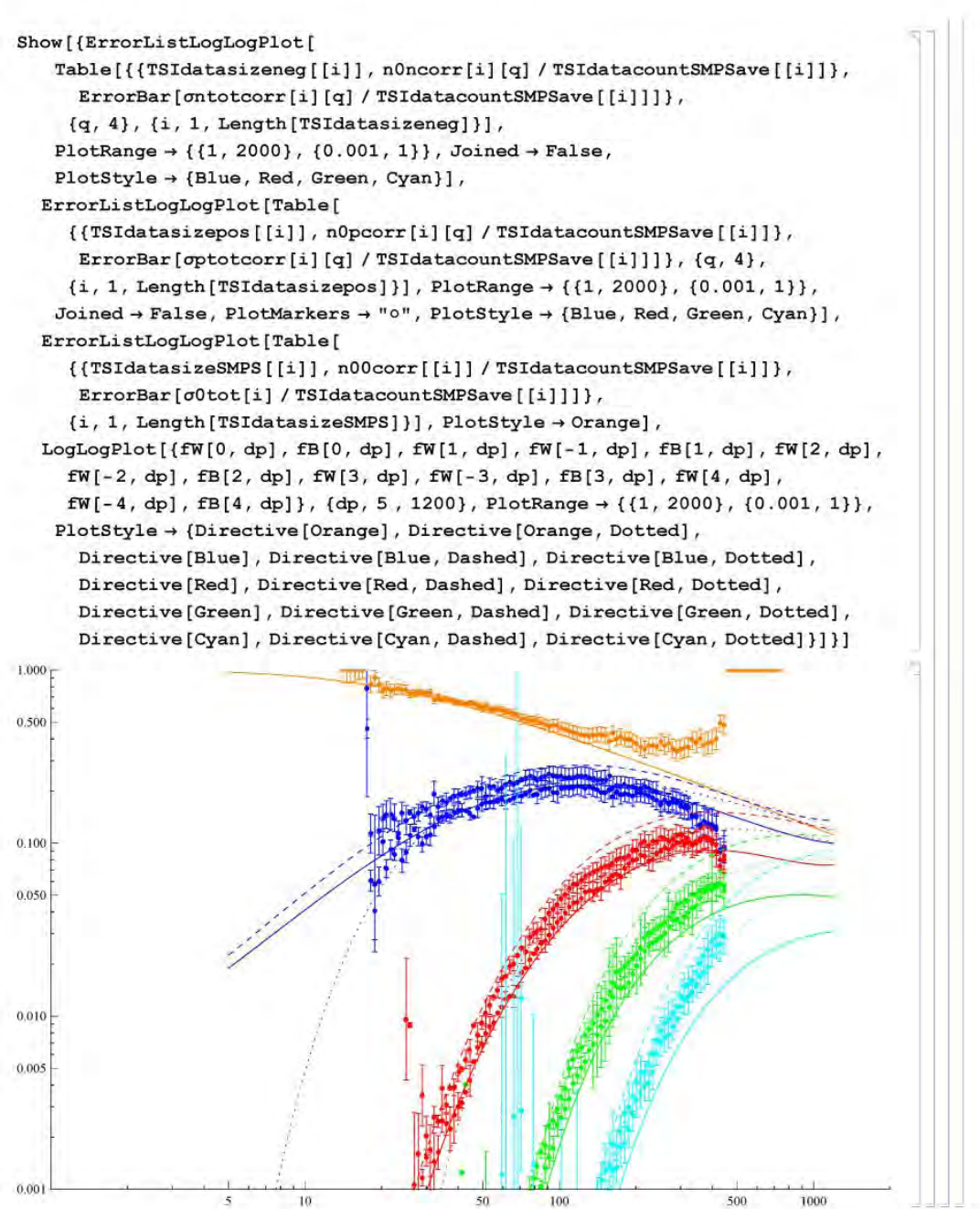


\section{VITA}

Matthew Simones was born in Shakopee, Minnesota on June 27, 1984. He attended public schools in Chaska, Minnesota and graduated from Chaska High School in 2002. He attended Hennepin Technical College where he received an A.A.S. degree in Medium/Heavy Truck Technology in 2005, during which time he practiced as a diesel technician. He went on to attend Minnesota State University, Mankato where he received a Bachelor of Science degree in Mechanical Engineering with a minor in Physics in May 2009. He continued his studies at Minnesota State University, Mankato and received a Master of Science degree in Mechanical Engineering in July 2011 with the experimental component of his thesis completed at the University of Missouri-Columbia. He then went on to pursue a Ph.D. in Nuclear Engineering at the University of Missouri-Columbia which he completed in December 2013. 